\title{
Molecular and Computational Bases for the Spirofuranone Formation in Setosusin Biosynthesis
}

Xingxing Wei, ${ }^{\dagger, \star}$ Taro Matsuyama, ${ }^{\S, \star}$ Hajime Sato, ${ }^{*, \S, \diamond}$ Dexiu Yan, ${ }^{\dagger}$ Pui Man Chan, ${ }^{\dagger}$ Kazunori Miyamoto, ${ }^{\S}$ Masanobu Uchiyama, ${ }^{*, \S, \#}$ and Yudai Matsuda*,†

†Department of Chemistry, City University of Hong Kong, Tat Chee Avenue, Kowloon, Hong Kong SAR, China ${ }^{\S}$ Graduate School of Pharmaceutical Sciences, The University of Tokyo, 7-3-1 Hongo, Bunkyo-ku, Tokyo 1130033, Japan

${ }^{\diamond}$ Interdisciplinary Graduate School of Medicine and Engineering, University of Yamanashi, 4-4-37 Takeda, Kofu, Yamanashi 400-8510, Japan

${ }^{*}$ Research Initiative for Supra-Materials (RISM), Shinshu University, 3-15-1 Tokida, Ueda, Nagano 386-8567, Japan

\section{Table of Contents}

Supplementary Materials and Methods

Supplementary Tables S1-S10

S9-S18

Supplementary Figures S1- S48

S19-S67

Cartesian Coordinates and Energies

S68-S86

Supplementary References

S87-S89 


\section{Supplementary Materials and Methods}

\section{General experimental procedures}

Organic solvents were purchased from Anaqua (Hong Kong) Co. Ltd., and other chemicals were purchased from Wako Chemicals Ltd., Thermo Fisher Scientific, Sigma-Aldrich, or J\&K Scientific Ltd., unless noted otherwise. $\left[1-{ }^{13} \mathrm{C}\right]$ acetate acid, [methyl- $\left.{ }^{13} \mathrm{C}\right]$ methionine, and sodium $\left[1,2-{ }^{13} \mathrm{C}_{2}\right]$ acetate were purchased from Cambridge Isotope Laboratories, Inc, and ${ }^{18} \mathrm{O}_{2}$ was obtained from Wuhan Niuruide Gas Co., Ltd. Oligonucleotide primers (Table S3) were purchased from Tech Dragon Limited. PCR was performed using a T100 ${ }^{\mathrm{TM}}$ Thermal Cycler (Bio-Rad Laboratories, Inc.) with Phanta Max Super-Fidelity DNA Polymerase (Vazyme Biotech Co., Ltd). Analytical HPLC was performed on a Dionex Ultimate 3000 UHPLC system (Thermo Scientific), using a Kinetex $2.6 \mu \mathrm{m} \mathrm{C}_{18} 100 \AA \AA$ column (2.1 i.d. x 100 mm; Phenomenex). Preparative HPLC was performed on a Waters 1525 Binary HPLC pump with a 2998 photodiode array detector (Waters Corporation), using a an XBridge BEH C18 OBD Prep Column (100 $\AA, 5 \mu \mathrm{m}, 19$ i.d. x 250 mm; Waters Corporation). Flash chromatography was performed using an Isolera Spektra One flash purification system (Biotage). NMR spectra were obtained at $600 \mathrm{MHz}\left({ }^{1} \mathrm{H}\right) / 150 \mathrm{MHz}\left({ }^{13} \mathrm{C}\right)$ with a Bruker Ascend Avance III HD spectrometer, and chemical shifts were recorded with reference to solvent signals $\left({ }^{1} \mathrm{H} \mathrm{NMR}: \mathrm{CDCl}_{3} 7.26 \mathrm{ppm}\right.$, $\mathrm{CD}_{3} \mathrm{OD} 3.31 \mathrm{ppm} ;{ }^{13} \mathrm{C}$ NMR: $\left.\mathrm{CDCl}_{3} 77.0 \mathrm{ppm}, \mathrm{CD}_{3} \mathrm{OD} 49.0 \mathrm{ppm}\right)$. HR-ESI-MS spectra were obtained with a micrOTOF-Q II mass spectrometer (Bruker Daltonics). Optical rotations were measured with P-2000 Digital Polarimeter (JASCO Corporation).

\section{Strains}

Aspergillus duricaulis CBS 481.65 was purchased from the Westerdijk Fungal Biodiversity Institute and used as a source for the cloning of each gene in the set cluster. Aspergillus oryzae NSAR1 (niaD $\left.{ }^{-}, s C^{-}, \Delta \arg B, \operatorname{ade} A^{-}\right)^{1}$ was utilized as the fungal heterologous expression host. Standard DNA engineering was performed with Escherichia coli DH5a (Takara Bio Inc).

\section{Construction of fungal transformation plasmids}

To construct fungal expression plasmids for $A$. oryzae, each gene in the set cluster was first amplified from the genomic DNA of $A$. duricaulis CBS 481.65 with the primers described in Table S3 and Table S4. Upon the constructions of the transformation plasmids, the database sequences of some set genes were manually revised (Table S2). Each amplified DNA fragment was then introduced into pTAex3 vector ${ }^{2}$ except for setA, setE, and $\operatorname{set} C$, whereas setA, setE, and $\operatorname{set} C$ were ligated into the pTAex $3 \mathrm{~A},{ }^{3} \mathrm{pUSA}^{4}{ }^{4}$ and $\mathrm{pUNA}^{5}$ vectors, respectively, using a ClonExpress Ultra One Step Cloning Kit (Vazyme Biotech Co., Ltd). For the construction of multigenecontaining plasmids, DNA fragments harboring the $a m y B$ promoter $(\operatorname{Pamy} B)$ and the $a m y B$ terminator $(\operatorname{Tamy} B)$ were amplified from the pTAex3-based plasmids, and further introduced into the already constructed single 
gene-containing vector or other vectors, $\mathrm{pAdeA}^{6}$ or pPTRI. ${ }^{7}$ Detailed methods for the construction of the plasmids used in this study are summarized in Table S4.

\section{Fungal transformation}

Transformation of $A$. oryzae was carried out by the previously reported protoplast-polyethylene glycol method, ${ }^{8}$ and the transformants created in this study and the plasmids used for the transformation are given in Table S5. Briefly, for the introduction of setA, setD, setE, set $G, \operatorname{set} H, \operatorname{set} J$, setI, setB, setK, setC, and setF, A. oryzae NSAR1 was initially transformed with the three plasmids, pTAex3A-setA+H, pUSA-setE+D+G and pAdeAsetJ+I. The resultant transformant was further transformed using the plasmids, pPTRI-setB+K and pUNAsetC $+\mathrm{F}$, to construct the 11-gene expressing transformant. To construct the transformants not expressing one or more genes, some of the plasmids were not used, or the plasmids containing only one gene were used upon performing the transformation.

\section{HPLC analysis of each product from $A$. oryzae transformants}

To analyze the metabolites from each A. oryzae transformant, the transformants were cultivated on a DPY agar plate [2\% dextrin, $1 \%$ hipolypepton (Nihon Pharmaceutical Co., Ltd.), $0.5 \%$ yeast extract, $0.5 \% \mathrm{KH}_{2} \mathrm{PO}_{4}, 0.05 \%$ $\mathrm{MgSO}_{4} \cdot 7 \mathrm{H}_{2} \mathrm{O}$, and $1.5 \%$ agar] for seven days at $30{ }^{\circ} \mathrm{C}$. A small piece of fungal mycelia and agar was cut from the plate, soaked in ethyl acetate, and extracted using an ultrasonic bath. The ethyl acetate layer was transferred to a new tube, and the solvent was removed using nitrogen gas flow.

The residue was dissolved in methanol and analyzed by HPLC, with a solvent system of $20 \mathrm{mM}$ formic acid (solvent A) and acetonitrile containing $20 \mathrm{mM}$ formic acid (solvent B), at a flow rate of $0.4 \mathrm{~mL} / \mathrm{min}$ and a column temperature of $40^{\circ} \mathrm{C}$. Separation was performed using a linear gradient from 10:90 (solvent B/solvent A) to $100: 0$ for $10 \mathrm{~min}, 100: 0$ for the following $3 \mathrm{~min}$, and a linear gradient from 100:0 to 10:90 within the following $2.0 \mathrm{~min}$, and then 10:90 for $2.5 \mathrm{~min}$ of equilibrium.

\section{Isolation of each metabolite}

To isolate each metabolite, A. oryzae transformants were cultivated either on DPY agar plates for seven days at $30^{\circ} \mathrm{C}$ or in DPY liquid medium at $30^{\circ} \mathrm{C} / 160 \mathrm{rpm}$ for three days. When cultivated on the agar plates, the resulting fungal cultures, including agar medium, were crushed into small pieces, soaked in ethyl acetate, and extracted twice using an ultrasonic bath. After filtration, ethyl acetate was removed in vacuo. When cultivated in the liquid medium, medium and mycelia were first separated by filtration. The medium was extracted with ethyl acetate, whereas the mycelia were extracted with acetone with sonication for one hour, concentrated, and reextracted with ethyl acetate. Both extracts were then combined. The resultant crude extract was fractionated by flash chromatography, and further purified by preparative HPLC. Purification methods for each compound are described in detail below. 


\section{Purification conditions for brevione B (2), brevione N (4), and colletopyrone (5):}

A. oryzae NSAR1 with setA, setD, setE, setG, setH, setJ, and setI was cultivated in 2 L of DPY liquid medium at $160 \mathrm{rpm}$ for three days. The extract from the transformant $(770 \mathrm{mg}$ ) was subjected to flash chromatography and eluted stepwise using a dichloromethane:ethyl acetate gradient (100:0 to 0:100). Fractions that contained 2, 4, and 5 were then purified by reverse-phase preparative HPLC ( $80 \%$ aqueous acetonitrile, $10.0 \mathrm{~mL} / \mathrm{min}$ ) to yield $124.1 \mathrm{mg}$ of $\mathbf{2}, 35.2 \mathrm{mg}$ of $\mathbf{4}$, and $2.3 \mathrm{mg}$ of $\mathbf{5}$.

\section{Purification conditions for brevione $\mathrm{R}(6)$ :}

$A$. oryzae NSAR1 with setA, setD, setE, setG, setH, setJ, setI, and setB was cultivated on 50 DPY agar plates (ca. $1 \mathrm{~L}$ ) for seven days. The extract from the transformant $(700 \mathrm{mg}$ ) was subjected to flash chromatography and eluted stepwise using a dichloromethane:ethyl acetate gradient (100:0 to 0:100). Fractions that contained 6 were then purified by reverse-phase preparative HPLC ( $55 \%$ aqueous acetonitrile, $10.0 \mathrm{~mL} / \mathrm{min}$ ) to yield $25 \mathrm{mg}$ of a colorless solid.

\section{Purification conditions for brevione S (7):}

A. oryzae NSAR1 with setA, setD, setE, setG, setH, setJ, setI, and setK was cultivated on 50 DPY agar plates (ca. $1 \mathrm{~L}$ ) for seven days. The extract from the transformant (490 mg) was subjected to flash chromatography and eluted stepwise using a dichloromethane:ethyl acetate gradient (100:0 to 0:100). Fractions that contained 7 were then purified by reverse-phase preparative HPLC ( $50 \%$ aqueous acetonitrile, $10.0 \mathrm{~mL} / \mathrm{min})$ to yield $50 \mathrm{mg}$ of a white solid.

\section{Purification conditions for brevione $L(3)$ :}

$A$. oryzae NSAR1 with setA, setD, setE, setG, setH, setJ, setI, setB, and setK was cultivated on 100 DPY agar plates (ca. 2 L) for seven days. The extract from the transformant (1190 mg) was subjected to flash chromatography and eluted stepwise using a dichloromethane:ethyl acetate gradient (100:0 to 0:100). Fractions that contained 3 were then purified by reverse-phase preparative HPLC (38\% aqueous acetonitrile, 10.0 $\mathrm{mL} / \mathrm{min}$ ) to yield $35 \mathrm{mg}$ of a white solid.

\section{Purification conditions for brevione T (8):}

$A$. oryzae NSAR1 with setA, setD, setE, setG, setH, setJ, setI, setB, setK, and $\operatorname{set} C$ was cultivated on 50 DPY agar plates $(c a .1 \mathrm{~L})$ for seven days. The extract from the transformant $(800 \mathrm{mg})$ was subjected to flash chromatography and eluted stepwise using a dichloromethane:ethyl acetate gradient (100:0 to 0:100). Fractions that contained $\mathbf{8}$ were then purified by reverse-phase preparative HPLC (43\% aqueous acetonitrile, 10.0 $\mathrm{mL} / \mathrm{min}$ ) to yield $18 \mathrm{mg}$ of a white solid. 


\section{Purification conditions for setosusin (1):}

$A$. oryzae NSAR1 with $\operatorname{set} A$, setD, $\operatorname{set} E, \operatorname{set} G, \operatorname{set} H, \operatorname{set} J$, setI, $\operatorname{set} B, \operatorname{set} K, \operatorname{set} C$, and $\operatorname{set} F$ was cultivated on 50 DPY agar plates $(c a .1 \mathrm{~L})$ for seven days. The extract from the transformant $(640 \mathrm{mg})$ was subjected to flash chromatography and eluted stepwise using a chloroform:ethyl acetate gradient (100:0 to 0:100). Fractions that contained 1 were then purified by reverse-phase preparative HPLC ( $70 \%$ aqueous acetonitrile, $10.0 \mathrm{~mL} / \mathrm{min})$ to yield $36.1 \mathrm{mg}$ of a yellowish solid.

\section{Bioconversion experiments}

To perform the bioconversion experiments, $A$. oryzae NSAR $1^{1}$ was transformed with pTAex3-setB, -setK, or setF. The resultant transformants were initially cultivated in $10 \mathrm{~mL}$ of DPY medium at $30{ }^{\circ} \mathrm{C}$ and $220 \mathrm{rpm}$ for three days and then transferred to $20 \mathrm{~mL}$ of DPY medium containing $25 \mathrm{mg} / \mathrm{L}$ of brevione B (2), brevione L (3), brevione $\mathrm{R}(6)$, brevione $\mathrm{S}(7)$, or brevione $\mathrm{T}(\mathbf{8})$. After cultivation at $30{ }^{\circ} \mathrm{C}$ and $160 \mathrm{rpm}$ for three days, medium and mycelium were separated by filtration. The medium was extracted with ethyl acetate, and the extract was analyzed by HPLC as described above.

\section{Synthesis of sodium $\left[1-{ }^{13} \mathrm{C},{ }^{18} \mathrm{O}_{2}\right]$ acetate}

The synthesis of sodium $\left[1-{ }^{13} \mathrm{C},{ }^{18} \mathrm{O}_{2}\right]$ acetate was performed according to a previously published method. ${ }^{9}$ Initially, $\left[1-{ }^{13} \mathrm{C}\right.$ ]acetic acid $\left(1 \mathrm{~g}, 16.4 \mathrm{mmol} ; 99 \%\right.$ isotopic purity), $\mathrm{H}_{2}{ }^{18} \mathrm{O}(1 \mathrm{~g}, 50.0 \mathrm{mmol} ; 90 \%$ isotopic purity), and $\mathrm{H}_{2} \mathrm{SO}_{4}(1 \mu \mathrm{L}, 0.0178 \mathrm{mmol})$ were mixed and incubated at $90{ }^{\circ} \mathrm{C}$ in a cylindrical pressure vessel for 24 hrs. After cooling down to room temperature, the $\mathrm{pH}$ of the reaction mixture was adjusted to $\sim 7$ using sodium hydroxide, followed by lyophilization to afford $1.78 \mathrm{~g}$ of white powder. The isotopic distribution of ${ }^{18} \mathrm{O}$ was estimated as $\sim 18 \%{ }^{16} \mathrm{O}_{2}, \sim 45 \%{ }^{16} \mathrm{O}^{18} \mathrm{O}$, and $\sim 37 \%{ }^{18} \mathrm{O}_{2}$ based on the ${ }^{13} \mathrm{C}$ NMR spectrum. ${ }^{1} \mathrm{H}$ NMR $\left(\mathrm{CD}_{3} \mathrm{OD}, 600\right.$ MHz): $\delta 1.89(\mathrm{~d}, J=5.9 \mathrm{~Hz}, 3 \mathrm{H}) ;{ }^{13} \mathrm{C}$ NMR $\left(\mathrm{CD}_{3} \mathrm{OD}, 150 \mathrm{MHz}\right): \delta 180.43,180.41,180.38,24.20(\mathrm{~d}, J=52.8$ $\mathrm{Hz}$ ); for NMR spectra see Figure S41 and Figure S42.

\section{In vivo labeling experiments}

For isotope incorporation experiments with $\left[\right.$ methyl $\left.-{ }^{13} \mathrm{C}\right]$ methionine, sodium $\left[1,2-{ }^{13} \mathrm{C}_{2}\right]$ acetate, or sodium $[1-$ ${ }^{13} \mathrm{C},{ }^{18} \mathrm{O}_{2}$ ]acetate, the $A$. oryzae transformant expressing set $A$, set $D$, set $E$, $\operatorname{set} G$, set $H$, setJ, setI, set $B$, setK, $\operatorname{set} C$, and $s e t F$ was cultivated on 25 DPY agar plates supplemented with $400 \mathrm{mg} / \mathrm{L}$ [methyl- ${ }^{13} \mathrm{C}$ ]methionine, $25 \mathrm{DPY}$ agar plates supplemented with $400 \mathrm{mg} / \mathrm{L}$ sodium $\left[1-{ }^{13} \mathrm{C},{ }^{18} \mathrm{O}_{2}\right]$ acetate, and $20 \mathrm{DPY}$ agar plates supplemented $500 \mathrm{mg} / \mathrm{L}$ sodium $\left[1,2-{ }^{13} \mathrm{C}_{2}\right]$ acetate, respectively, at $30{ }^{\circ} \mathrm{C}$ for seven days. Labeled setosusin was extracted and purified as described above to yield $7.0 \mathrm{mg}, 5.3 \mathrm{mg}$, and $13.8 \mathrm{mg}$ of yellowish solid from [methyl$\left.{ }^{13} \mathrm{C}\right]$ methionine, sodium $\left[1,2-{ }^{13} \mathrm{C}_{2}\right]$ acetate, and sodium $\left[1-{ }^{13} \mathrm{C},{ }^{18} \mathrm{O}_{2}\right]$ acetate, respectively.

To incorporate ${ }^{18} \mathrm{O}_{2}$, the $A$. oryzae transformant harboring $s e t F$ was cultivated at $30{ }^{\circ} \mathrm{C} / 160 \mathrm{rpm}$ in $150 \mathrm{~mL}$ DPY 
liquid medium containing $66.7 \mathrm{mg} / \mathrm{L}$ brevione $\mathrm{T}(\mathbf{8})$ under ${ }^{18} \mathrm{O}_{2}$ atmosphere. After three-day cultivation, medum and mycelia were separated by filtration, and the filtrated medium was loaded onto Sep-Pak C18 plus short cartridge (Waters Corporation) and eluted stepwise using a water:methanol gradient (100:0 to 0:100). Fractions that contained setosusin (1) were then purified by reverse-phase preparative HPLC (58\% aqueous acetonitrile, $10.0 \mathrm{~mL} / \mathrm{min}$ ) to afford $5.5 \mathrm{mg}$ of yellowish solid.

\section{In vitro enzymatic reaction and labeling experiment}

Mycelia from a two-day culture of $A$. oryzae transformant with $\operatorname{set} F$ were ground in liquid nitrogen, suspended in $25 \mathrm{~mL}$ of the reaction buffer (1.0 mM DTT, $1.0 \mathrm{mM}$ EDTA, 20\% glycerol, $100 \mathrm{mM}$ Tris-HCl, $\mathrm{pH} 7.5$ ), and incubated on ice for $1 \mathrm{hr}$. The cell debris was then removed by centrifugation, and the resultant supernatant was concentrated by ultrafiltration to $\sim 5 \mathrm{~mL}$.

The enzymatic reaction of SetF with brevione $\mathrm{T}(\mathbf{8})$ was performed in a reaction mixture containing $188 \mu \mathrm{L}$ of crude enzyme extract, $2.5 \mathrm{mM}$ NADPH, and $0.5 \mathrm{mM} \mathrm{8}$, in a final volume of $200 \mu \mathrm{L}$. Crude enzyme extract obtained in the same manner from A. oryzae NSAR1 was used as a negative control. To investigate the incorporation of $\mathrm{H}_{2}{ }^{18} \mathrm{O}_{2}, 100 \mu \mathrm{L}$ of the crude enzyme extract was replaced with $\mathrm{H}_{2}{ }^{18} \mathrm{O}_{2}(90 \%$ isotopic purity) or $\mathrm{H}_{2}{ }^{16} \mathrm{O}_{2}$. After an incubation at $30{ }^{\circ} \mathrm{C}$ for $12 \mathrm{~h}$, the reaction mixture was extracted with ethyl acetate and analyzed by LC-MS.

\section{Computational details}

All calculations were carried out using the Gaussian 16 package. ${ }^{10}$ Structure optimizations were done with the M06-2X ${ }^{11}$ density functional theory method and the $6-31+G(d, p)$ basis set without any symmetry restrictions. Vibrational frequency calculations at the same level of theory with optimization were performed to verify that each local minimum has no imaginary frequency and that each TS has only a single imaginary frequency. Intrinsic reaction coordinate (IRC) calculations ${ }^{12}$ for all TSs were performed with GRRM $11^{13}$ based on Gaussian 16.

\section{Bioconversion experiments with SetF variants}

To allow for a fair comparison of the activities of each SetF variant, the $s e t F$ wild type gene and its variant genes were integrated onto the $s C$ locus of $A$. oryza $e^{14}$ by homologous recombination, respectively. To this end, a new fungal transformation vector, designated as pTAex3-HR, was created as follows. Upstream and downstream regions of the target site $(\sim 1 \mathrm{~kb}$ each) were first amplified from the genomic DNA of $A$. oryzae NSAR1, and then inserted into pTAex3 vector, in which $\arg B$ (selection marker)-PamyB-TamyB is flanked by the upstream/downstream regions (Figure S8). Primer sequences and detailed method for the construction of pTAex3-HR are summarized in Table S3 and Table S4. Subsequently, setF variants were constructed by PCR 
methods, using pairs of the mutation primers (Table S3), and then ligated into the pTAex3-HR vector that had been digested by SmaI (Table S4).

The transformation of A. oryzae was performed by CRISPR-Cas9-mediated DNA double-strand break and repair by homologous recombination. To this end, the components for the dual Cas9-gRNA system, namely Cas9 protein, CRISPR RNAs (crRNAs), and transactivating CRISPR RNA (tracrRNA), were purchased from Integrated DNA Technologies; two separate crRNAs were designed at the promoter region of $s C$, respectively (protospacer sequences: 5'-GAGTTTCTCTTAAAGTAATA-3'; 5'-CAAGGACCACGTCCTTCAAC-3'). The Cas9 ribonucleoprotein (RNP) complexes were assembled as reported ${ }^{15}$ and used for the fungal transformation along with the pTAex3-HR-based plasmid constructed above.

The bioconversion experiments and analysis of the resultant metabolites were performed as described above, using brevione $\mathrm{T}(\mathbf{8})$ as a substrate. Two individual transformants were used for each variant.

\section{Analytical data}

Setosusin (1). Yellowish solid; $[\alpha]^{21}{ }_{\mathrm{D}}+31.7$ (c 0.98, $\left.\mathrm{MeOH}\right) ;{ }^{1} \mathrm{H}$ NMR $\left(\mathrm{CDCl}_{3}, 600 \mathrm{MHz}\right): \delta 5.75$ (brs, $\left.1 \mathrm{H}\right)$, $4.87(\mathrm{t}, J=4.0 \mathrm{~Hz}, 1 \mathrm{H}), 3.09$ (d, $J=4.1 \mathrm{~Hz}, 2 \mathrm{H}), 2.88(\mathrm{~d}, J=15.8 \mathrm{~Hz}, 1 \mathrm{H}), 2.44$ (d, $J=15.8 \mathrm{~Hz}, 1 \mathrm{H}), 2.29$ (dd, $J=9.3,7.6 \mathrm{~Hz}, 1 \mathrm{H}), 2.26(\mathrm{~s}, 3 \mathrm{H}), 2.10(\mathrm{~s}, 3 \mathrm{H}), 2.05(\mathrm{brd}, J=10.1 \mathrm{~Hz}, 1 \mathrm{H}), 1.95$ (m, 2H), 1.93 (s, 3H), 1.84 (m, 1H), $1.70(\mathrm{~s}, 3 \mathrm{H}), 1.64(\mathrm{~m}, 1 \mathrm{H}), 1.59(\mathrm{~m}, 1 \mathrm{H}), 1.54(\mathrm{~s}, 3 \mathrm{H}), 1.51(\mathrm{~s}, 3 \mathrm{H}), 1.51(\mathrm{~m}, 1 \mathrm{H}), 1.20(\mathrm{~s}, 3 \mathrm{H}), 0.93$ $(\mathrm{s}, 3 \mathrm{H}) ;{ }^{13} \mathrm{C} \mathrm{NMR}\left(\mathrm{CDCl}_{3}, 150 \mathrm{MHz}\right): \delta 199.7,185.7,170.5,170.4,169.9,130.5,129.9,110.6,90.2,87.0,85.6$, 70.3, 50.3, 43.9, 41.0, 39.1, 35.3, 34.5, 32.6, 31.7, 24.0, 22.9, 21.1, 21.0, 19.6, 16.7, 15.1, 15.0, 5.8; for NMR spectra see Figure S9 and Figure S10; HRMS (ESI) $m / z$ : $[\mathrm{M}+\mathrm{Na}]^{+}$Calcd for $\mathrm{C}_{29} \mathrm{H}_{38} \mathrm{O}_{8} \mathrm{Na}$ 537.2459; Found 537.2463. The NMR data are in good agreement with the reported data. ${ }^{16}$

Brevione $B$ (2). Yellowish solid; $[\alpha]^{22}{ }_{\mathrm{D}}+75.9\left(c 0.94, \mathrm{CHCl}_{3}\right) ;{ }^{1} \mathrm{H} \mathrm{NMR}\left(\mathrm{CDCl}_{3}, 600 \mathrm{MHz}\right): \delta 5.67$ (brs, $\left.1 \mathrm{H}\right)$, $3.04(\mathrm{~d}, J=15.8 \mathrm{~Hz}, 1 \mathrm{H}), 2.90(\mathrm{~d}, J=15.8 \mathrm{~Hz}, 1 \mathrm{H}), 2.58$ (ddd, $J=15.8,11.1,7.3 \mathrm{~Hz}, 1 \mathrm{H}), 2.41$ (ddd, $J=15.8$, 6.8, 3.7 Hz, 1H), $2.22(\mathrm{~s}, 3 \mathrm{H}), 2.05(\mathrm{~m}, 2 \mathrm{H}), 1.95(\mathrm{ddd}, J=13.1,7.2,3.7 \mathrm{~Hz}, 1 \mathrm{H}), 1.93(\mathrm{~s}, 3 \mathrm{H}), 1.74(\mathrm{dd}, J=$ 10.0, 6.9 Hz, 1H), $1.64(\mathrm{brs}, 3 \mathrm{H}), 1.55(\mathrm{~m}, 2 \mathrm{H}), 1.50(\mathrm{~m}, 1 \mathrm{H}), 1.31(\mathrm{~m}, 2 \mathrm{H}), 1.08(\mathrm{~s}, 3 \mathrm{H}), 1.08(\mathrm{~s}, 3 \mathrm{H}), 1.06(\mathrm{~s}$, $3 \mathrm{H}), 0.95$ (s, 3H); ${ }^{13} \mathrm{C} \mathrm{NMR}\left(\mathrm{CDCl}_{3}, 150 \mathrm{MHz}\right): \delta 217.4,171.4,162.4,160.5,131.7,127.9,103.1,99.8,99.6$, 55.1, 47.4, 47.0, 40.9, 38.9, 36.5, 34.0, 31.8, 28.6, 26.4, 23.2, 21.4, 18.9, 18.4, 17.2, 16.0, 15.5, 9.7; for NMR spectra see Figure S11 and Figure S12; HRMS (ESI) $m / z$ : $[\mathrm{M}+\mathrm{H}]^{+}$Calcd for $\mathrm{C}_{27} \mathrm{H}_{37} \mathrm{O}_{4}$ 425.2686; Found 425.2679. The NMR data are in good agreement with the reported data. ${ }^{17}$

Brevione L (3). White solid; $[\alpha]^{22}{ }_{\mathrm{D}}+17.6$ (c 1.01, $\left.\mathrm{MeOH}\right) ;{ }^{1} \mathrm{H}$ NMR $\left(\mathrm{CDCl}_{3}, 600 \mathrm{MHz}\right): \delta 5.62$ (brd, $J=4.7$ $\mathrm{Hz}, 1 \mathrm{H}), 3.81(\mathrm{~d}, J=7.3 \mathrm{~Hz}, 1 \mathrm{H}), 3.19$ (d, $J=15.2 \mathrm{~Hz}, 1 \mathrm{H}), 3.00$ (d, $J=15.7 \mathrm{~Hz}, 1 \mathrm{H}), 2.92$ (dd, $J=15.5,7.4$ $\mathrm{Hz}, 1 \mathrm{H}), 2.89$ (d, $J=15.8 \mathrm{~Hz}, 1 \mathrm{H}), 2.63(\mathrm{dd}, J=11.7,5.2 \mathrm{~Hz}, 1 \mathrm{H}), 2.19$ (s, 3H), 2.16 (brd, $J=19.0 \mathrm{~Hz}), 2.00$ $(\mathrm{dd}, J=12.6,1.5 \mathrm{~Hz}, 1 \mathrm{H}), 1.96(\mathrm{~m}, 1 \mathrm{H}), 1.90(\mathrm{~s}, 3 \mathrm{H}), 1.66(\mathrm{brd}, J=13.7 \mathrm{~Hz}, 1 \mathrm{H}), 1.62(\mathrm{~s}, 3 \mathrm{H}), 1.55(\mathrm{td}, J=$ 
13.4, 3.2 Hz, 1H), 1.50 (dt, $J=13.2,3.5 \mathrm{~Hz}, 1 \mathrm{H}), 1.47(\mathrm{~s}, 3 \mathrm{H}), 1.43(\mathrm{~s}, 3 \mathrm{H}), 1.38(\mathrm{td}, J=13.2,4.1 \mathrm{~Hz}, 1 \mathrm{H}), 1.11$ (s, 3H), 0.93 (s, 3H); ${ }^{13} \mathrm{C} \mathrm{NMR}\left(\mathrm{CDCl}_{3}, 150 \mathrm{MHz}\right): \delta 172.6,171.2,162.1,160.4,131.4,127.7,103.0,99.6,99.2$, 86.1, 68.3, 49.3, 44.6, 40.6, 38.8, 38.8, 34.4, 31.5, 28.6, 23.6, 22.9, 21.1, 18.4, 17.1, 16.4, 15.5, 9.6; for NMR spectra see Figure S13 and Figure S14; HRMS (ESI) $m / z$ : $[\mathrm{M}+\mathrm{H}]^{+}$Calcd for $\mathrm{C}_{27} \mathrm{H}_{37} \mathrm{O}_{6}$ 457.2585; Found 457.2599. The NMR data are in good agreement with the reported data. ${ }^{18}$

Brevione N (4). Yellowish oil; $[\alpha]^{23}{ }_{\mathrm{D}}+71.4\left(c\right.$ 1.00, $\left.\mathrm{CHCl}_{3}\right)$; for NMR data see Table $\mathrm{S} 6$ and Figure $\mathrm{S} 15$ to Figure S20; HRMS (ESI) $m / z$ : $[\mathrm{M}+\mathrm{H}]^{+}$Calcd for $\mathrm{C}_{27} \mathrm{H}_{39} \mathrm{O}_{4}$ 427.2843; Found 427.2846.

Colletopyrone (5). Colorless solid; ${ }^{1} \mathrm{H}$ NMR $\left(\mathrm{CDCl}_{3}, 600 \mathrm{MHz}\right): \delta 11.16(\mathrm{~s}, 2 \mathrm{H}), 3.54(\mathrm{~s}, 2 \mathrm{H}), 2.24(\mathrm{~s}, 6 \mathrm{H})$, $1.95(\mathrm{~s}, 6 \mathrm{H}) .{ }^{13} \mathrm{C} \mathrm{NMR}\left(\mathrm{CDCl}_{3}, 150 \mathrm{MHz}\right): \delta 169.5,168.7,156.8,109.6,101.7,19.1,17.2,9.8$; for NMR spectra see Figure S21 and Figure S22; HRMS (ESI) $m / z$ : $[\mathrm{M}+\mathrm{H}]^{+}$Calcd for $\mathrm{C}_{15} \mathrm{H}_{17} \mathrm{O}_{6}$ 293.1020; Found 293.1024. The NMR data are in good agreement with the reported data. ${ }^{19}$

Brevione $R$ (6). Colorless solid; $[\alpha]^{22}{ }_{\mathrm{D}}+86.6\left(c 0.99, \mathrm{CHCl}_{3}\right)$; for NMR data see Table S7 and Figure S23 to Figure S28; HRMS (ESI) $m / z$ : $[\mathrm{M}+\mathrm{H}]^{+}$Calcd for $\mathrm{C}_{27} \mathrm{H}_{37} \mathrm{O}_{5}$ 441.2636; Found 441.2645.

Brevione S (7). White solid; $[\alpha]^{22}{ }_{\mathrm{D}}+60.7\left(c 0.92, \mathrm{CHCl}_{3}\right)$; for NMR data see Table S8 and Figure S29 to Figure S34; HRMS (ESI) $m / z$ : $[\mathrm{M}+\mathrm{H}]^{+}$Calcd for $\mathrm{C}_{27} \mathrm{H}_{37} \mathrm{O}_{5}$ 441.2636; Found 441.2645.

Brevione $T$ (8). White solid; $[\alpha]^{22}{ }_{\mathrm{D}}+9.1\left(c 1.03, \mathrm{CHCl}_{3}\right)$; for NMR data see Table $\mathrm{S} 9$ and Figure $\mathrm{S} 35$ to Figure S40; HRMS (ESI) $m / z$ : $[\mathrm{M}+\mathrm{H}]^{+}$Calcd for $\mathrm{C}_{29} \mathrm{H}_{39} \mathrm{O}_{7}$ 499.2690; Found 499.2694. 
Table S1. Annotation of each gene in the set cluster.

The set cluster from Aspergillus duricaulis CBS 481.65

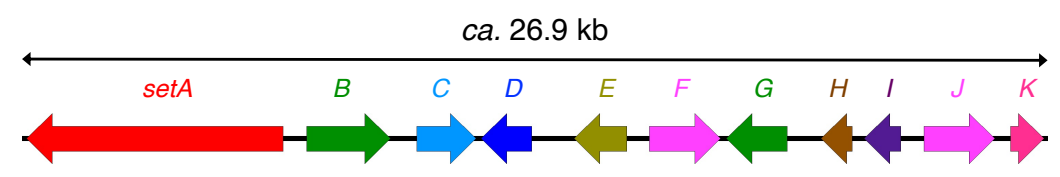

\begin{tabular}{|c|c|c|c|c|c|}
\hline Gene & Protein ID & acids (base pairs) & Protein homologue (origin) & Similarity/ldentity (\%) & Proposed function \\
\hline setA & 270789 & $2244(6790)$ & DpmpA (Macrophomina phaseolina) & $56 / 40$ & $\begin{array}{l}\text { non-reducing polyketide synthase } \\
\text { (SAT-KS-AT-PT-ACP-ACP-CMeT) }\end{array}$ \\
\hline setB & 270787 & $664(1955)$ & AusC (Aspergillus nidulans) & $69 / 53$ & FAD-dependent monooxygenase \\
\hline $\operatorname{setC}$ & 255878 & $501(1567)$ & AdrJ (Penicillium rubens) & $56 / 36$ & acetyltransferase \\
\hline $\operatorname{set} D$ & 270785 & $332(1141)$ & DpchC (Colletotrichum higginsianum) & $67 / 54$ & prenyltransferase \\
\hline setE & 270783 & $356(1260)$ & DpmpD (Macrophomina phaseolina) & $80 / 65$ & geranylgeranyl pyrophosphate synthase \\
\hline setF & 264610 & $512(1884)$ & Pyr3 (Aspergillus fumigatus) & $61 / 41$ & cytochrome P450 monooxygenase \\
\hline $\operatorname{setG}$ & 264609 & $476(1608)$ & Cdml (Talaromyces verruculosus) & $65 / 50$ & FAD-dependent monooxygenase \\
\hline setH & 270779 & $241(785)$ & PtmB (Penicillium simplicissimum) & $60 / 37$ & terpene cyclase \\
\hline setl & 264607 & $257(967)$ & CdmF (Talaromyces verruculosus) & $72 / 55$ & short-chain dehydrogenase/reductase \\
\hline setJ & 264606 & $513(1867)$ & AdrA (Penicillium rubens) & $64 / 43$ & cytochrome P450 monooxygenase \\
\hline setK & 264605 & $297(894)$ & CdmA (Talaromyces verruculosus) & $79 / 61$ & $\mathrm{Fe}(\mathrm{II}) / \mathrm{KG}$-dependent dioxygenase \\
\hline
\end{tabular}

Note: Protein IDs are as designated in the JGI database. The sequences of $\operatorname{set} A$, $\operatorname{set} B$, and $\operatorname{set} H$ were revised in this study (see Table S2).

Table S2. Revised DNA and protein sequences. Highlighted in magenta are the regions predicted for an intron in this study.

\begin{tabular}{|c|c|c|}
\hline Gene & Revised DNA sequence & Predicted/revised protein sequence \\
\hline setA & $\begin{array}{l}\text { ATGTCCACAAGATTACCAGCAGGCGTATTTTGTGGCGCACAAAGTATCCCATCATCTGATATTTGCGAGTCGATT } \\
\text { CGTCTGTGCCTCCGTAAAGACCCTGGCCTTCAACATCTTGCACAGACAGTCGTTGAACTCCCCGCTTGTGGT } \\
\text { CAAGGCTCACTGAAACGCACGCTTTCTGCGATGCTGCAGTTGGCGATCCCTTGAAACCCCTCAGTGACTGGAT } \\
\text { AACTTCAGATAACCCTCGGTCTCTCACAATCCCTGAATCTTAACCAACGTCCTCCTTGCACCATTGACTGTCGT } \\
\text { TTTGCAGCTGGTGCAGTACACCCAGTATCTGAGGGAACAGCATCATGCGGCCATATCTCATGAGCAGGTTTTGC } \\
\text { AGTCCGTCTCATCCCCTAGTGCGGGTGTCCAGGGGTTATGCACTGGGTTTCTGACGGCAACGGCGCTAGCTTG } \\
\text { TTCGCGAGACTTGGCTGAGGTGAACACGAATGGTAAGGAGCGATTCGTCTGGCAATGTGTATCGGTGAGCA } \\
\text { GTCGATGCAGATGAGCGGAAAAGGGAGGGTGGAAACCGCACACAGTGTCTGGTTGCGCATGGGAGTCCGCA } \\
\text { CCTTGACTGTCGGGAGGTGATGGCGGCGTTGAGCAGTACCCAAGAGTATGCACTAGAAAGATGAATTGTGAA } \\
\text { CTTCCCCAGCTAACACTGGTTGTTGAGGTATATGTCTCGGTCAAATTGGACGCGCACCGAGTGACCATCACTG } \\
\text { CAGTGGATGAGGACGTCCCATCGCTCAGCCAAGATCTCATACAACATGGGATCAAACTGAAACCTCTGTCATTG } \\
\text { AAAGGGCGATATCATAATGCTGTGCACGAGGAGATCGTCGCTGCCCTGAAGGAGGTATGCAATGCTGAACCAG } \\
\text { GTCTACGGTTTCCTGAAGAGAGCAAACCACTCGTCCCTCTGCGGTGTAACACTACTGGAGATCTCGTGCAACC } \\
\text { CGGGGAGATCCTGCATGATGTCTGCCTGCGGTCTCTTCTGATGGAACCAGCCGATTGGTATTCCACAGTGACA } \\
\text { GCCTCAATCGCAGGGATGACACATTCTGGTTCGGAGAATGCGTTGTCTCATACGGTGTTGGAGTTGGGGTTCG } \\
\text { TGCCTTGTCTGCCGCGCTCATTGGTGACACGTTCCTGCTTGGACGTGCTCTCTGCCTCCCTTGACGCGGATGA } \\
\text { TCGGCCTTTCTCTTATCCAGATCATTCCATCGCAATAGTCGGAGCAGCAGGTAGGTTCGCAGGAGCCGAAAGCT }\end{array}$ & $\begin{array}{l}\text { MSTRLPAGVFCGAQSIPSSDICESIRLCLRKDPGLQ } \\
\text { HLAQTVVELPALWSRLTETHAFCDAAVGDPLKPLS } \\
\text { DWITSDNPRSLTIPESLPNVLLAPLTVVLQLVQYTQ } \\
\text { YLREQHHAAISHEQVLQSVSSPSAGVQGLCTGFLT } \\
\text { ATALACSRDLAEVNTNGEVAIRLAMCIGAAVDADER } \\
\text { KREGGNRTQCLVAHGSPHLDCREVMAALEQYPRV } \\
\text { YVSVKLDAHRVTITAVDEDVPSLSQDLIQHGIKLKPL } \\
\text { SLKGRYHNAVHEEIVAALKEVCNAEPGLRFPEESK } \\
\text { PLVPLRCNTTGDLVQPGEILHDVCLRSLLMEPADW } \\
\text { YSTVTASIAGMTHSGSENALSHTVLELGFVCLPR } \\
\text { SLVTRSCLDVLSASLDADDRPFSYPDHSIAIVGAAG } \\
\text { RFAGAESLDQLWNLIRTGSSTVSTCTQDGSMPVR } \\
\text { DSQTQTGNYLSNVDAFDRGLFGYSPNEAQFMDP } \\
\text { QHRVALQVAYEAVEGAGYLSPGSCPRKDIGCYVGI } \\
\text { ATSDYEANVACHRASAFSFTGTARSFVSGRISHYF } \\
\text { GWTGPSVAIDTACSSSAVAITMACKELMTKGCKMA } \\
\text { LAGGVNISNCRTTRNLAAANFLSASSGPCRSFDE }\end{array}$ \\
\hline
\end{tabular}


TGGATCAGCTGTGGAATCTGATCCGAACTGGATCTTCCACGGTCTCGACATGTACGCAAGACGGGTCAATGCC AGTGAGAGATAGCCAGACTCAAACAGGGAACTACCTCTCTAACGTGGACGCCTTTGATCGTGGATTGTTTGGCT ACTCACCCAACGAAGCCCAATTCATGGACCCGCAGCACAGAGTAGCGTTACAAGTTGCCTACGAAGCCGTTGA AGGGGCAGGATATCTGAGCCCGGGTTCTTGTCCGCGAAAGGATATCGGCTGCTATGTGGGCATTGCCACGTCT GACTACGAGGCTAATGTCGCATGTCACCGCGCGAGTGCCTTTTCCTTTACGGGAACAGCTCGATCGTTCGTGA GTGGACGCATCAGTCACTACTTTGGTTGGACGGGCCCCTCCGTGGCCATTGATACAGCATGTTCCTCCTCAGC GGTCGCCATTACTATGGCCTGCAAGGAACTCATGACCAAGGGCTGCAAAATGGCCCTTGCAGGTGGCGTCAAT ATTATCAGCAACTGTCGTACGACGAGAAATCTGGCGGCGGCCAACTTCCTCAGTGCTTCTTCAGGCCCGTGCC GCTCCTTTGACGAGGCCGCGGATGGTTTCTCTCGTGGAGAAGGGTGTGGATTTATCTTACTCAAAAGGCTCGA TGATGCTCTGGCGGAAAACGACCAGATTCTCGGCGTCATCGTTGGTACTGCCACCAACCAGAACATCAACTGC GATCCTATCACGGTCCCCGTCCACGAGTCCCAGGTCGATGTCTACCAGCGTAGCTTAGCCGCGTCGGGTATTG ATTCCCTACGTGTGTCCTATGTTGAGGCGCACGGCACCGGGACAACGAAAGGGGATCCCATTGAGTATCACAG CATTCGGCAGGTCTTCGGAGGCCGGAATGAGCGGTCTCAACCCCTGTATATCGGCTCCGTGAAAGCCAACATC GGGCATACCGAGGCAGCTTCGGGAGTTGCTAGCGTGCTCAAGGTGCTCTTGATGATGCGGCACGGTGAACTT CCCCCTCAGGGCAACTTCACCAGGTTGAATCCGGCGATAGCGAGTCTCGAGTCGGATCAGATCAGCATTCCTA CTGCTCTCCAGCCATGGAAAAGCAATTTCCGCGTGGCTAGTATTAACAACTACGGCGCTTCCGGGAATAACACG GCCATGATAGTGTGCCAGCCTCCGAATGTGCTACAGCACCCCAAGTGGCAGGAGGACACGGCGCTTCGCTAT CCCTTTTTGCTATCCGCAAACACGAAAACCAGTCTGCGGAAGATGTGTGAATCGCTCCAGCAATTCATTGCTCT TGCCCTGCAGCGCACCGCACGACGTCCGCTTCGTCTGGCCGACCTGGCCATCTGGATCGGATCAAAGCAGAA TCAACGGCTGCGGTACCGCATCGCATTTGATGCGGGATCAATTCCCGAACTGCAGGATCATTTGCATCGATACA TCACAGATGATGGCGGCACTGCTGTGATCCCGGGACCGTCGTCGACCACCGCAAGGCCGGTAGTCATGGTCT TCGCGGGCCAGACTGGCAATACAGTCCACATCAACAAGGATGCGTACAATTGCAGTGCTCTGCTTAAATCACAT CTGGACCGCTGTGATGCTGTCCTTCGGGAGACGGGCCGTCCAAGTCTGTTTCCCAACATATTCCAATCTAAGC CCGAAGCGGATGTGAGTTTGTTGCATTGCATGTTGTTCGCAGCGCAATACTCGTGCGCGATGGCCTGGATTGAT GCAGGGTTGCCTGTGCAGAGGCTTATTGGGCACAGCATTGGCCAGCTGACGGCGCTGTGCGTTGCCGGTTGC ATCAGTCTTCGGGATGCCATCGCCTTGATCGCGGGACGGGCGTCTTTGATCAAGGAGCAATGGGGGAGTGAG ACCGGGGTGATGCTCGCTGTGAATATGGACCGATTAAGCACCGAGACACTGGTGAGATTGGTTGCTGAAGGAG ATCCGGATTGCGCAGTCGAGATTGCATGTTTTAATGCCCCGGCCCGTCACATCTTGGTAGGCACCGAGGCAGC CATTCGCAAAGTCGAGGGCCTATTGAGTGAATACGGCAATCCGCGACGGTTAAAAACCACACATGGCTTTCATT CGAGGCTGGCAGACTCCATTATGAGGTCATATGAGGCCATCGCCCAGAGCATCTCCTATCACGAGCCCACCATT CCCATTGAGACTTGCTCCCCTGGAAGCAGCTGGGCTCGGGTGTCTGCAGAGCTTGTTGCCCAACAGACTCGC CAGCCAGTGTATTTCGTCGATGCCATTAGGCGTATCGAAAAGCGTTTCGGTCCCTGTGTCTGGCTGGAGGCCG GGACCGGATCCTCGGGTATTACTCTCGCTCGACATGCTCTTCAGAACTCACAGAGTCATTCATTCCACGCTGTA CAACTGGCTTCGTCAGAACCGATTGCCTCCCTCCAACACACGACTCAACAGCTCTGGTCAGAAGGGATGGCC GTGCAGTTCTGGTTGTACCATCAAATTGGCAGATCATCTTCCTGTTTATCCTGGTGTGTCCCTGGATATCAGTTC GATGAATCGCGTCATTGGGTTTCGTATAAAGAACCAGAGAAAGCAGCGGTTGAATATGAGGATTCCCACACTGG CTCCGCCTGCACCAGTCTGATCACCTTGATTACTCCCCCCGCGATAGGTGGCGACCAGTCCCAGGTGGCAAAA TTCTCCATCGACACGCATAATGAAGAATACCAGGGATGGCTCAGCTGCCGCGTAGTTCTGGGGTCGTATCTATG TTCGATAGCATCGTACGTCGAGCTGGCTGCTCGTGCCGCTATGCTCTTATCCCGAGGCTTGGAACACCATCACT GCCTTAAGGTCTCAAATCTATCCCTCTGCGCTCCTCTGGGCATACAGGTAGATAGTCCCCTTACACTTACCTTGC GGTCGACCACTGTAACGAGTGAGTGGGAATTCTCCATTGACAGCGACATCCAGCACGCCAAGGGACATATTGG CATCATGCCTATGCCCAGCACGTCAGCGCTATCTTCTGCCCTACAACGCTTAATTGACTATCGATATTGCCAGCG CATCCTTGCTGATCCAGATGCATCATCTTTACGAGGTTCACTGGCGTACAAGATCCTTGAAAGAGTAGTGGAGT GTGCCCCGTCATATCAGGGGATTCAGTCCACCACAATCCGAGACTTGGAGGCCGTTGCTCATGTTATACTACCT CCGGCTGCACACAGTTTACAAGGGCAAGGGCAAGAAAAGAGCGCTTGCAATTGGCAGACTTTGGATCACTTTA TCCTGGCGGCGGAGATGCACATATTGAGTCAAAAGGAGTGCAGTCACCATGATGTCTTTGTGTGCTCGGAGTT GACAGAAATCATACCGTTCGTCACCGCTCCATTGGACGGAAGAGGTCCTTGGATGGTATATACCCAACTATCGT GGGCCAATGAGCACACCATCCTCTGTGACACTCTAGTATTTGATACCGCCACCCAGCAGTCCATCATGGCATTG CTGGGGGCCCGTTACACCAGAGTCTCTACCAGCTCGCTGCAGAAAGCTATTTCCCTTGCAAGCAGTCATCAGG AGCAGCAGCACCAGCAGAACATGCTCCCTGTATCTCCTTGCCATCAGCCTGTTACTACCGCCAGACCTCTCGC TCCTCTTATTCCTCCTACCCCCGTAACCCCAACTGGAGAAGCTGTGGAAGAGAAGCTCAAGTATATTGTCTCCG ACCTAACCGGCATACCACCGTCTCAGCTCTCACCTATGACCTCTTTGGCTGAGCTTGGAGTGGACTCGCTCAC GACAATCGAGCTGCGGAGATCGTTCAAGGAGAGCTTTCAAGTCGATCTGCCTAACGATATCGACAACGGCCATT ACACGATAGGTGATTTGTCTGCTGCTGCTGCTGCTGCTGCTGCCGTGAATCTCTCACAGCTACAGCCTCAGCT CTCAGGAAAGCTCAAACTTTGCCAGGAGATGTTTCTACCACCAACCGGATTGATCACACCCTCCAGTGGCCCA ACCACTCCGTCATCACCTGGAGGTGGAAGGACAAACACATCGGAAACCTTGAAACACAGCTCAGTGTGTGAAA TTATTACCCGGCTTATCAGGATCGTCGCAGCGCAGCTCAACTGCCCTGACAAGATTGTTCCGAGCATCCAACTA CAACATCTGGGGCTTGATTCCCTCGGGGCGATGGACCTGGAAGCCGAGCTGGCCAAGGCTTTTGGACGGAAG CTGAACCTACAGAAAATGTCTCCCGCATCGACATTCGGTGACTTAATGGATCTGGTTCTGTCGTCGCATGGGCA GCCGTCGTTGGACCAGATATGCACACCTACGGGGATCAGTACAGCGTCTGGCACAGGTAGTCCCAGTATCCTC TTATCCAACCCCAGGTTCCTCTTCGAGGCGCTCTCCTGGTTCGCAGGTATTCGACAAACCTACAGCCAGTTCGC TCGGATGGCGGGATTCTCAGGGTTCTACACGCACGTCTATCCACGACAGATGCGTCTGCTCACGTCCTATATCG TCGACGCGTTCGGAGCACTAGGGTGTAAGCTGCCTGACATTCCCGCGGGACAACTCGTGCCGCGAATCGCTC ATATCCCCAGACACGAGAAGCTCGTAGACCACTACTATCGCATCCTGAAGGACAGCGGTCTTATCCAGAGAGAC AAGGTCAACGGCAGTACCTGGATCCGCACATCCAATGCCATCCATCCGGCGCCTGCGTCTGAAACGTATCAAG CGGTCTGGACCGACTTCCCAGCCTATCGTCCAGAACACGAATTACTGCACTGCACCGGTACTCGTCTCGCCGA TTGTCTCTCCGGACAGGCGGACCCTCTCTACCTCCTCTTCCAAGACCCGAAGGCCCACGAGCTCCTCGAAAA GGTGTACGCCGACTGTCCAATGTTCAATACAGGCACGCGTCTACTCAGCCACTTCCTCCATCAAGTCGCGCCG CCATCCAGCCCTGAACCGCTTCGGATCCTAGAGCTAGGCGCCGGCACAGGCGGTACAACCAAGCCAGTCCTC
AADGFSRGEGCGFILLKRLDDALAENDQILGVIVGT ATNQNINCDPITVPVHESQVDVYQRSLAASGIDSLR VSYVEAHGTGTTKGDPIEYHSIRQVFGGRNERSQ PLYIGSVKANIGHTEAASGVASVLKVLLMMRHGELP PQGNFTRLNPAIASLESDQISIPTALQPWKSNFRVA SINNYGASGNNTAMIVCQPPNVLQHPKWQEDTAL RYPFLLSANTKTSLRKMCESLQQFIALALQRTARRP LRLADLAIWIGSKQNQRLRYRIAFDAGSIPELQDHL HRYITDDGGTAVIPGPSSTTARPVVMVFAGQTGNT VHINKDAYNCSALLKSHLDRCDAVLRETGRPSLFP NIFQSKPEADVSLLHCMLFAAQYSCAMAWIDAGLP VQRLIGHSIGQLTALCVAGCISLRDAIALIAGRASLIK EQWGSETGVMLAVNMDRLSTETLVRLVAEGDPDC AVEIACFNAPARHILVGTEAAIRKVEGLLSEYGNPR RLKTTHGFHSRLADSIMRSYEAIAQSISYHEPTIPIE TCSPGSSWARVSAELVAQQTRQPVYFVDAIRRIEK RFGPCVWLEAGTGSSGITLARHALQNSQSHSFHA VQLASSEPIASLQHTTQQLWSEGMAVQFWLYHQIG RSSSCLSWCVPGYQFDESRHWVSYKEPEKAAVE YEDSHTGSACTSLITLITPPAIGGDQSQVAKFSIDTH NEEYQGWLSCRVVLGSYLCSIASYVELAARAAMLL SRGLEHHHCLKVSNLSLCAPLGIQVDSPLTLTLRST TVTSEWEFSIDSDIQHAKGHIGIMPMPSTSALSSAL QRLIDYRYCQRILADPDASSLRGSLAYKILERVVEC APSYQGIQSTTIRDLEAVAHVILPPAAHSLQGQGQE KSACNWQTLDHFILAAEMHILSQKECSHHDVFVCS ELTEIIPFVTAPLDGRGPWMVYTQLSWANEHTILCD TLVFDTATQQSIMALLGARYTRVSTSSLQKAISLAS SHQEQQHQQNMLPVSPCHQPVTTARPLAPLIPPT PVTPTGEAVEEKLKYIVSDLTGIPPSQLSPMTSLAE LGVDSLTTIELRRSFKESFQVDLPNDIDNGHYTIGD LSAAAAAAAAVNLSQLQPQLSGKLKLCQEMFLPPT GLITPSSGPTTPSSPGGGRTNTSETLKHSSVCEIIT RLIRIVAAQLNCPDKIVPSIQLQHLGLDSLGAMDLEA ELAKAFGRKLNLQKMSPASTFGDLMDLVLSSHGQ PSLDQICTPTGISTASGTGSPSILLSNPRFLFEALS WFAGIRQTYSQFARMAGFSGFYTHVYPRQMRLLT SYIVDAFGALGCKLPDIPAGQLVPRIAHIPRHEKLVD HYYRILKDSGLIQRDKVNGSTWIRTSNAIHPAPASE TYQAVWTDFPAYRPEHELLHCTGTRLADCLSGQA DPLYLLFQDPKAHELLEKVYADCPMFNTGTRLLSH FLHQVAPPSSPEPLRILELGAGTGGTTKPVLEQLR SRKINFHYTFSDISAALVARARDQLAGYQGPGAAID FTVVNIERPPLHEHVQAYDVVISANCVHATKDLGVS CANICALLRPKGMLCLLELTRDVYWLDGVFGLLDG WWRFDDGREHALVDERQWKDCLQRAGFAHVDW TDDESRESDVFRLIFAMKQ 


\begin{tabular}{|c|c|c|}
\hline & $\begin{array}{l}\text { GAACAGCTCAGGTCTCGAAAGATAAACTTCCACTACACATTCAGCGATATTTCCGCAGCATTGGTGGCACGTGC } \\
\text { TCGGGACCAACTGGCGGGCTATCAGGGTCCCGGAGCAGCAATCGATTTCACAGTCGTCAATATTGAGCGGCCC } \\
\text { CCGCTACACGAACATGTGCAAGCGTATGATGTGGTGATATCGGCCAACTGCGTCCATGCAACCAAGGATCTGG } \\
\text { GAGTCTCGTGCGCGAATATCTGCGCCCTGCTGCGGCCGAAAGGGATGCTATGTCTTTTGGAGCTTACGCGTGA } \\
\text { TGTGTACTGGTTGGATGGTGTCTTGGGGCTATTGGATGGGTGGTGGCGATTTGATGATGGGCGAGAGCATGCG } \\
\text { CTTGTGGATGAACGACAGTGGAAGGATTGCTTGCAAAGAGCTGGATTTGCACATGTTGATTGGACGGACGATG } \\
\text { AATCGAGGGAATCAGATGTTTTTAGGTTGATTTTTGCCATGAAGCAGTAG }\end{array}$ & \\
\hline setB & $\begin{array}{l}\text { ATGGTCTCTACTATAACAGCCCCCCTCCAGCCTTCTGTCACTGTCTGTCCATGTCACGCAGCTGACTGTAAGCA } \\
\text { CAAATACCAGGCTCGGATAGAAAAGAAATATGAGTTAGAACGCCATATCCAGCTACAGAGTCGTGGGACAGTTG } \\
\text { CCGATGTGGCCGTCGACTCTACTGAGAGGCTTCGATGCCTCGGGCAAACCCCTGGCTCCAATCGGATGCCA } \\
\text { ATGAGCAGCGGTCTCCAAACCAACCAGAACCCGAGCCCAAACAGGCCATCACAAAGTGCTAATCCTCGGGG } \\
\text { CAGGATTCGGCGGGCTTTTGTTTGCCGTACGCCTGATCCAGACGGGAGCATTTACCGCGGACGACCTCCTTTT } \\
\text { GGTCGACAAAGCCGGAGGCTTTGGAGGTACCTGGTACTGGACCGATATCCGGGACTTCGCTGCGACGTGGA } \\
\text { AAGTTACATATACCTTCCTCTCCTGGAAGAGACGGGATACATGCCACGCGAGAAATATGCATCGGGAGCGGAGA } \\
\text { TCCGCCGGTACGCCAATCTCATTGCGCGCACCTGGAGGCTCACAGGGCGTGCACTGTTCCAAGCAACCATCC } \\
\text { AGCGGTTGGATTGGGACGACACCAAGCACATCTGGACTGCAACGGGCGTCCGATCCGGGGGGGAGCTGTC } \\
\text { CAGCTCAGTGCGGACTTTGTCCTCCTGGCCCCTGGCAGTTACGCTGGTGGAAGGTCCCCGACTTCCCGCAT } \\
\text { ATCAACAGATACCAAGGGAATGTATTCCATACGTCTCGTTGGGACTACAATTGCACCGGGGGCACCCCAGATAG } \\
\text { TCCGCATATGGACCAACTACGAGACAAGCAGGTGGGAATCGTGGGCACTGGGGCACGGCTGTCCAGGTGAT } \\
\text { TCCCCAGCTGGCGCAGTATAGCAAGCAGCTACTTGTGCTTCAACGCACCCCATCACCAGTCGATCAGCGCAAC } \\
\text { AATTGTCCCACGGATCCTGCGTGGTGGAAGTCCATGATCGAGGCTGAGGGCCCGGGGTGGCAGCGACGGCG } \\
\text { GATGGAAAACTTCAACGCCTTCACCTCATACCAACAGCCGCCACCACCGGTCAATCTGGTGGGCGATGCCTGG } \\
\text { ACTGCAATGCCTTCATTCAGTGTCGTGACCGGGACCGAACAGGCGCAGAAGCCGGATTACATGGACCGAATCA } \\
\text { AGGCCATCGATATGGCCCGGCAGGAGCGGATCCGAGCCCGAGTCGGGGACTCGGTCGAGGACGAGGTAGTA } \\
\text { GCGGAGGCTTTACACCGTGGTATCCTGGCTGGTGCAAGCGTCCGTGCTTTCATGACGAGTACCTTGCTGCAT } \\
\text { TCAACCAGCCAAATGTAAAGCTCATTGATATTCGCAAGAGCGGGATAACCAGTTTTACGGAGAAGGGCTTGCTG } \\
\text { GTGGACGGCATCGAACATGAACTGGATGTCGTGGTCTTAGTACGGGCTACTCGATCCCGCTGGGGCCCATGA } \\
\text { GCCCCGGTGACGTGCCCACATTGACATCACCGGTCGTCATGGGCTGACGATGGATAACAAATGGAAGGATGG } \\
\text { ACTGGCCACGCTTCACGGGATGATGACGAGTGATCTGCCCAATCTCTTTTTCACTGGGCATAACCAGGCGGGT } \\
\text { GTCTGTGCCAACCAGATGTACGTCTTGGTCCAGCAATCTGCCCACGTTGCTTTTATCTTGGCTGAAGCTGTTCG } \\
\text { GCAAGCTGGCAGTATCGAGGGCCAGGAGGATGCAAAGGGGATAGTCATCGAGCCAACTGCGGACGGGGAAAA } \\
\text { AGGTTGGGCTCTGCAGGTGCTGTCGCGCTTAGCCCCCGGTCAGCAGCCGCCATTCTGGATTGCACACCGGG } \\
\text { GTACTACAACGGCGAGGGTGCATTGTCCAAAATGAACGATTTGAAGCCAGAGGAACAGGCGAAGATGGCTCTT } \\
\text { TTGTCTAACTGGGGGAGGGGATCGCGAACTATGTCGAATCGCTCGAGAACTGGAGGAGTCAGGGACAGTTG } \\
\text { CCTGGTTTGAAGCTGCAGTTTGGACATGACTAG }\end{array}$ & $\begin{array}{l}\text { MVSTITAPLQPSVTVCPCHAADCKHKYQARIEKKY } \\
\text { ELERHIQLQSRGTVADVAVDSTERLRCLGQDPWL } \\
\text { QSDANEQRSPNQPEPEPKTGHHKVLILGAGFGGL } \\
\text { LFAVRLIQTGAFTADDLLLVDKAGGFGGTWYWNRY } \\
\text { PGLRCDVESYIYLPLLEETGYMPREKYASGAEIRRY } \\
\text { ANLIARTWRLTGRALFQATIQRLDWDDTKHIWTATG } \\
\text { VRSGGAAVQLSADFVLLAPGSYAGGKVPDFPHINR } \\
\text { YQGNVFHTSRWDYNCTGGTPDSPHMDQLRDKQV } \\
\text { GIVGTGATAVQVIPQLAQYSKQLLVLQRTPSPVDQR } \\
\text { NNCPTDPAWWKSMIEAEGPGWQRRRMENFNAFT } \\
\text { SYQQPPPPVNLVGDAWTAMPSFSVVTGTEQAQKP } \\
\text { DYMDRIKAIDMARQERIRARVGDSVEDEVVAEALT } \\
\text { PWYPGWCKRPCFHDEYLAAFNQPNVKLIDIRKSGI } \\
\text { TSFTEKGLLVDGIEHELDVVVLSTGYSIPLGPMSPG } \\
\text { GRAHIDITGRHGLTMDNKWKDGLATLHGMMTSDL } \\
\text { PNLFFTGHNQAGVCANQMYVLVQQSAHVAFILAEA } \\
\text { VRQAGSIEGQEDAKGIVIEPTADGEKGWALQVLSR } \\
\text { LAPRSAAAILDCTPGYYNGEGALSKMNDLKPEEQA } \\
\text { KMALLSNWGEGIANYVESLENWRSQGQLPGLKLQ } \\
\text { FGHD* }\end{array}$ \\
\hline setH & $\begin{array}{l}\text { ATGAATTCGATGGACTTCTCCAAAGCACCTCCTGGGTTCCAGGAGGTGAGATGGATCTCCAATATCCTGCTCAC } \\
\text { TACCCTCGCATCCGGCTGGCTGGTCTGTTACATAGCCACAATCCGCACAGCCTTCCGTGACCGAGCTTGCTGG } \\
\text { ATGCCCTTAATTCCATTATCCTGCAATCTATCATGGGAGCTGGTCTACGTCTTCTCTATCCTCCACCACGTCTTC } \\
\text { CCATTGTCACCTTCTGGCTCATGCTAAACCTCGTGGTGGTCTACGTGGCCGTGAGATTTGCCTCAGAGCCTGG } \\
\text { TCAGCACACTGCCGCCGCGTCAAAGCGGAGTCTTGCCGTCTTCTTTGTCTTGGGGGCTCTTTTCTGGGGTGCT } \\
\text { GGGCATGTCGCTTTGATTTCGCAGGTTGGACCTCTTACAGCGTTCTATTATGGTGGATTGGGATGCCAGATCAT } \\
\text { GACAAGTGCGACGGCGTTGTGAGGCTGGTGGATCGTGGTTGTACGACGGGCGCGTCTTACATTATCTGGTAA } \\
\text { GAGAAATTGTTCCCCGATCAGGTTTCTGATTCCAATGTTGATGCTTGCGACACAGGGTCGCTCGGGTCATCGG } \\
\text { CACCTCGAGTGCCTTACTCGGCGTGTATTTAGGGCACATTACTGGCCAGAACTTTGGGCCTGGGCTGTAAAC } \\
\text { CCGCTGATGCATTGGTGTACTGCTGCATTTGTCATCCTGGATGGCTTGTATGGGGTGTGTTTTTGGTATATCCGT } \\
\text { CAATCAGAGACGTCGCAGCAGAAAGTTCTTGACGCTAAGAAGGATTGA }\end{array}$ & $\begin{array}{l}\text { MNSMDFSKAPPGFQEVRWISNILLTTLASGWLVCY } \\
\text { IATIRTAFRDRACWMPLIPLSCNLSWELVYVFLYPP } \\
\text { PRLPIVTFWLMLNLVVVYVAVRFASEPGQHTAAAS } \\
\text { KRSLAVFFVLGALFWGAGHVALISQVGPLTAFYYG } \\
\text { GLGCQIMTSATALCRLVDRGCTTGASYIIWVARVIG } \\
\text { TSSALLGVYFRAHYWPELWAWAVNPLMHWCTAAF } \\
\text { VILDGLYGVCFWYIRQSETSQQKVLDAKKD** }\end{array}$ \\
\hline
\end{tabular}


Table S3. Primers used in this study.

\begin{tabular}{|c|c|}
\hline Primer & Sequence $\left(5^{\prime}\right.$ to $\left.3^{\prime}\right)$ \\
\hline setA-F & AAACGAATTCGAGCTCGGTACCCATGTCCACAAGATTACCAGCAGGCG \\
\hline setA-F1 & CATTCGAGGCTGGCAGACTCCATTATGAGG \\
\hline setA-R & TTCACGAGCTACTACAGATCCCCTGCTACTGCTACTGCTTCATGGC \\
\hline setA-R1 & GAGTCTGCCAGCCTCGAATGAAAGCCATG \\
\hline setB-F & AATTCGAGCTCGGTACCCGGGATGGTCTCTACTATAACAGCC \\
\hline setB-R & GAGCTACTACAGATCCCCTAGTCATGTCCAAACTGCAGCTTC \\
\hline setC-F & AATTCGAGCTCGGTACCCATCATGGGCGCCGCCAAGTCCAAAG \\
\hline setC-R & GAGCTACTACAGATCCCCTTCTGGGTAGATTCTATGGAAGGTTTG \\
\hline setD-F & AATTCGAGCTCGGTACCCATGACTACCGCAACAGGGGAAAC \\
\hline setD-R & GAGCTACTACAGATCCCCAGGATTATGAACGAATTGTCAGCTC \\
\hline setE-F & AATTCGAGCTCGGTACCCAAAATGCTGCCCGCAACGCCCCTCT \\
\hline setE-R & GAGCTACTACAGATCCCCTACTCCTTCAATTCCAGAAGGTCGAG \\
\hline setF-F & AATTCGAGCTCGGTACCCATGCTGCTAGTCTCACTTGGAAGC \\
\hline setF-R & GAGCTACTACAGATCCCCTGTTACAGTTTGATCTCCTCCTGTCG \\
\hline setG-F & AATTCGAGCTCGGTACCCATGGAGGTCGAGCAGAAGCGGT \\
\hline setG-R & GAGCTACTACAGATCCCCACGTCTATGAAACTAGTAGTAACCG \\
\hline setH-F & AATTCGAGCTCGGTACCCATGAATTCGATGGACTTCTCCAAAGC \\
\hline setH-R & GAGCTACTACAGATCCCCACTCTCCATCAATCCTTCTTAGCG \\
\hline setl-F & AATTCGAGCTCGGTACCCATGGGGACCCTCCAGGATCGCGTGAGAA \\
\hline setl-R & GAGCTACTACAGATCCCCTAAATTGTGGACCAGCCGTCGTC \\
\hline setJ-F & AATTCGAGCTCGGTACCCATGCACCACACCGGGTTCAATAC \\
\hline setJ-R & GAGCTACTACAGATCCCCTCACAGTGTCATCTCCTCCTGCC \\
\hline setK-F & AATTCGAGCTCGGTACCCATGACCATCCCAGCACTTGACGC \\
\hline setK-R & GAGCTACTACAGATCCCCTACTACATGCTCCCCTGAATTTC \\
\hline InF-pTAex3_BInI-F & ACGCGCAATTCCTAGCCCATCATGGTGTTTTGATC \\
\hline InF-pTAex3_BInI-R & TTGACCGAATCCTAGGTAAGATACATGAGCTTCGG \\
\hline InF-pUSA_BamHI-F & TTATAGGAAAGGATCCCCATCATGGTGTTTTGATC \\
\hline InF-pUSA_BamHI-R & TGACTCTAGAGGATCGTAAGATACATGAGCTTCGG \\
\hline InF-pAdeA_Spel-F & TAGAGGATCTACTAGTCAAGAGCAGAATGTGAACG \\
\hline InF-pAdeA_Spel-R & AATCCATATGACTAGTGATACATGAGCTTCGGTG \\
\hline In-pPTRI_Smal-F & CTCTAGAGGATCCCCATCATGGTGTTTTGATC \\
\hline In-pPTRI_Smal-R & TCGAGCTCGGTACCCGTAAGATACATGAGCTTCGG \\
\hline InF-pUNA_Xbal-F & GAAAGGATCCTCTAGCCCATCATGGTGTTTTGATC \\
\hline InF-pUNA_Xbal-R & GCAGGTCGACTCTAGGTAAGATACATGAGCTTCGG \\
\hline InF-linker-F1 & GCTCGCGAGCGCGTTCCACTGCATCATCAGTCTAG \\
\hline InF-linker-R1 & AACGCGCTCGCGAGCAAGTACCATACAGTACCGCG \\
\hline sC-5'-F & CGGGTTGTTTGAGCTAGCAATGGC \\
\hline$s C-5^{\prime}-\mathrm{R}$ & CAAGAAGTACAGTTACATTCGAAGGTGAC \\
\hline sC-3'-F & GGTCTTGAATGGTGGGATATTTTGTCC \\
\hline sC-3'-R & GGAGTCAGCCACCGAGTCAACTTTC \\
\hline pTAex3-F1 & GAATGTAACTGTACTTCTTGTTGCATGCCTGCAGGTTCGAC \\
\hline pTAex3-R1 & ATATCCCACCATTCAAGACCCATGGTGGGGTAACCAAGGTTG \\
\hline pTAex3-F2 & TTGACTCGGTGGCTGACTCCACCACAGGGATATGATGGCACC \\
\hline pTAex3-R2 & TTGCTAGCTCAAACAACCCGGCCGTCGTTTTACAACGTCGT \\
\hline setF-K303A-F & CAGCTCGCGGGCGTAGCTGCAGCCGT \\
\hline setF-K303A-R & TACGCCCGCGAGCTGGGCCACAGCTACATCATAG \\
\hline setF-K303R-F & CAGCTCAGGGGCGTAGCTGCAGCCGT \\
\hline setF-K303R-R & TACGCCCCTGAGCTGGGCCACAGCTACATCATAG \\
\hline setF-S311A-F & CGCCGTCCAGGAAGTACTAACCCAGAC \\
\hline setF-S311A-R & ACTTCCTGGACGGCGCCGACGGCTGCAGCTAC \\
\hline setF-S370A-F & GCCTGCTACAATCTGTAAGGAGATGCCATTAGAATATC \\
\hline setF-S370A-R & CAGATTGTAGCAGGCTTAAGACGCAGAGTCTC \\
\hline setF-T371A-F & GCCTAGTGCAATCTGTAAGGAGATGCCATTAGAATATC \\
\hline setF-T371A-R & CAGATTGCACTAGGCTTAAGACGCAGAGTCTC \\
\hline
\end{tabular}


Table S4. Plasmids constructed in this study and PCR conditions for the amplification of the inserts for the plasmid constructions.

\begin{tabular}{|c|c|c|c|c|c|}
\hline Plasmid & Inserts & Primer 1 & Primer 2 & PCR Template & Vector \\
\hline pTAex3A-setA & $\begin{array}{l}1^{\text {st }} \text { half of } \operatorname{set} A \\
2^{\text {nd }} \text { half of } \operatorname{set} A\end{array}$ & $\begin{array}{l}\text { setA-F } \\
\text { setA-F1 }\end{array}$ & $\begin{array}{l}\text { setA-R1 } \\
\text { setA-R }\end{array}$ & gDNA & pTAex3A digested with Smal \\
\hline pTAex3-setB & setB & setB-F & setB-R & gDNA & pTAex3 digested with Smal \\
\hline pTAex3-setD & setD & setD-F & setD-R & gDNA & pTAex3 digested with Smal \\
\hline pTAex3-setF & setF & setF-F & setF-R & gDNA & pTAex3 digested with Smal \\
\hline pTAex3-setG & $\operatorname{set} G$ & setG-F & setG-R & gDNA & pTAex3 digested with Smal \\
\hline pTAex3-setH & setH & setH-F & setH-R & gDNA & pTAex3 digested with Smal \\
\hline pTAex3-setl & setl & setl-F & setl-R & gDNA & pTAex3 digested with Smal \\
\hline pTAex3-setJ & setJ & setJ-F & setJ-R & gDNA & pTAex3 digested with Smal \\
\hline pTAex3-setK & setK & setK-F & setK-R & gDNA & pTAex3 digested with Smal \\
\hline pUSA-setE & setE & setE-F & setE-R & gDNA & pUSA digested with Smal \\
\hline pUNA-setC & setC & setC-F & setC-R & gDNA & pUNA digested with Smal \\
\hline pUNA-setF & setF & setF-F & setF-R & gDNA & pUNA digested with Smal \\
\hline pTAex3A-setA+H & PamyB-setH-TamyB & InF-pTAex3_BInI-F & InF-pTAex3_BInI-R & pTAex3-setH & pTAex3A-setA digested with BInl \\
\hline pUSA-setE+D+G & $\begin{array}{l}\text { PamyB-setD-TamyB } \\
\text { PamyB-setG-TamyB }\end{array}$ & $\begin{array}{l}\text { InF-pUSA_BamHI-F } \\
\text { InF-linker-F1 }\end{array}$ & $\begin{array}{l}\text { InF-linker-R1 } \\
\text { InF-pUSA_BamHI-R }\end{array}$ & $\begin{array}{l}\text { pTAex3-setD } \\
\text { pTAex3-setG }\end{array}$ & pUSA-setE digested with $\mathrm{BamHI}$ \\
\hline pAdeA-setJ+l & $\begin{array}{l}\text { PamyB-setJ-TamyB } \\
\text { PamyB-setl-TamyB }\end{array}$ & $\begin{array}{l}\text { InF-pAdeA_Spel-F } \\
\text { InF-linker-F1 }\end{array}$ & $\begin{array}{l}\text { InF-linker-R1 } \\
\text { InF-pAdeA_Spel-R }\end{array}$ & $\begin{array}{l}\text { pTAex3-setJ } \\
\text { pTAex3-setl }\end{array}$ & pAdeA digested with Spel \\
\hline pPTRI-setB & PamyB-setB-TamyB & InF-pPTRI_Smal-F & InF-pPTRI_Smal-R & pTAex3-setB & pPTRI digested with Smal \\
\hline pPTRI-setK & PamyB-setK-TamyB & InF-pPTRI_Smal-F & InF-pPTRI_Smal-R & pTAex3-setK & pPTRI digested with Smal \\
\hline pPTRI-setB+K & $\begin{array}{l}\text { PamyB-setB-TamyB } \\
\text { PamyB-setK-TamyB }\end{array}$ & $\begin{array}{l}\text { In-pPTRI_Smal-F } \\
\text { InF-linker-F1 }\end{array}$ & $\begin{array}{l}\text { InF-linker-R1 } \\
\text { In-pPTRI_Smal-R }\end{array}$ & $\begin{array}{l}\text { pTAex3-setB } \\
\text { pTAex3-setK }\end{array}$ & pPTRI digested with Smal \\
\hline pUNA-setC+F & PamyB-setF-TamyB & InF-pUNA_Xbal-F & InF-pUNA_Xbal-R & pTAex3-setF & pUNA-setC digested with $X b a l$ \\
\hline pTAex3-HR & $\begin{array}{l}s C \text { promoter front side } \\
\text { part of pTAex3 (1) } \\
s C \text { promoter rear side } \\
\text { part of pTAex3 (2) }\end{array}$ & $\begin{array}{l}\text { sC-5'-F } \\
\text { pTAex3-F1 } \\
\text { sC-3'-F } \\
\text { pTAex3-F2 }\end{array}$ & $\begin{array}{l}\text { sC-5'-R } \\
\text { pTAex3-R1 } \\
\text { sC-3'-R } \\
\text { pTAex3-R2 }\end{array}$ & $\begin{array}{l}\text { gDNA } \\
\text { pTAex3 } \\
\text { gDNA } \\
\text { pTAex3 }\end{array}$ & $\mathrm{N} / \mathrm{A}$ \\
\hline pTAex3-HR-setF & setF & setF-F & setF-R & pTAex3-setF & pTAex3-HR digested with Smal \\
\hline pTAex3-HR-setF (K303A) & $\begin{array}{l}1^{\text {st }} \text { half of } \operatorname{setF}(\mathrm{K} 303 \mathrm{~A}) \\
2^{\text {nd }} \text { half of } \operatorname{setF}(\mathrm{K} 303 \mathrm{~A})\end{array}$ & $\begin{array}{l}\text { setF-F } \\
\text { setF-K303A-F }\end{array}$ & $\begin{array}{l}\text { setF-K303A-R } \\
\text { setF-R }\end{array}$ & pTAex3-setF & pTAex3-HR digested with Smal \\
\hline pTAex3-HR-setF (K303R) & $\begin{array}{l}1^{\text {st }} \text { half of } \operatorname{setF}(\mathrm{K} 303 \mathrm{R}) \\
2^{\text {nd }} \text { half of } \operatorname{setF}(\mathrm{K} 303 \mathrm{R})\end{array}$ & $\begin{array}{l}\text { setF-F } \\
\text { setF-K303R-F }\end{array}$ & $\begin{array}{l}\text { setF-K303R-R } \\
\text { setF-R }\end{array}$ & pTAex3-setF & pTAex3-HR digested with Smal \\
\hline pTAex3-HR-setF (S311A) & $\begin{array}{l}1^{\text {st }} \text { half of } \operatorname{setF}(\mathrm{S} 311 \mathrm{~A}) \\
2^{\text {nd }} \text { half of } \operatorname{setF}(\mathrm{S} 311 \mathrm{~A})\end{array}$ & $\begin{array}{l}\text { setF-F } \\
\text { setF-S311A-F }\end{array}$ & $\begin{array}{l}\text { setF-S311A-R } \\
\text { setF-R }\end{array}$ & pTAex3-setF & pTAex3-HR digested with Smal \\
\hline pTAex3-HR-setF (S370A) & $\begin{array}{l}1^{\text {st }} \text { half of } \operatorname{setF}(\mathrm{S} 370 \mathrm{~A}) \\
2^{\text {nd }} \text { half of } \operatorname{setF}(\mathrm{S} 370 \mathrm{~A})\end{array}$ & $\begin{array}{l}\text { setF-F } \\
\text { setF-S370A-F }\end{array}$ & $\begin{array}{l}\text { setF-S370A-R } \\
\text { setF-R }\end{array}$ & pTAex3-setF & pTAex3-HR digested with Smal \\
\hline pTAex3-HR-setF (T371A) & $\begin{array}{l}1^{\text {st }} \text { half of } \operatorname{setF}(T 371 \mathrm{~A}) \\
2^{\text {nd }} \text { half of } \operatorname{setF}(\mathrm{T} 371 \mathrm{~A})\end{array}$ & $\begin{array}{l}\text { setF-F } \\
\text { setF-T371A-F }\end{array}$ & $\begin{array}{l}\text { setF-T371A-R } \\
\text { setF-R }\end{array}$ & pTAex3-setF & pTAex3-HR digested with Smal \\
\hline
\end{tabular}

Table S5. Aspergillus oryzae transformants constructed in this study.

\begin{tabular}{|l|l|l|}
\hline Strain & Host strain & Plasmids used for transformation \\
\hline A. oryzae/setADEGHJI & A. oryzae NSAR1 & pTAex3A-setA+H, pUSA-setE+G+H, pAdeA-setJ+I \\
\hline A. oryzae/setADEGHJIB & A. oryzae/setADEGHJI & pPTRI-setB \\
\hline A. oryzae/setADEGHJIK & A. oryzae/setADEGHJI & pPTRI-setK \\
\hline A. oryzae/setADEGHJIBK & A. oryzae/setADEGHJI & pPTRI-setB+K \\
\hline A. oryzae/setADEGHJIBKC & A. oryzae/setADEGHJIBK & pUNA-setC \\
\hline A. oryzae/setADEGHJIBKF & A. oryzae/setADEGHJIBK & pUNA-setF \\
\hline A. oryzae/setADEGHJIBKCF & A. oryzae/setADEGHJIBK & pUNA-setC+F \\
\hline
\end{tabular}


Table S6. NMR data for brevione N (4).

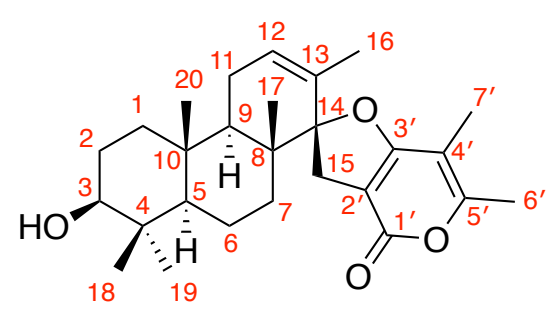

\begin{tabular}{|c|c|c|c|c|c|}
\hline position & $\delta_{c}$, type & $\delta_{\mathrm{H}}$, mult. $(\mathrm{J}$ in $\mathrm{Hz})$ & HMBC correlation & COSY correlation & NOESY correlation \\
\hline \multirow[t]{2}{*}{1} & $38.2, \mathrm{CH}_{2}$ & $1.05(a), \operatorname{td}(12.9,3.4)$ & $9,10,20$ & $\mathrm{H}-2 \mathrm{a}, \mathrm{H}-2 \beta$ & $\mathrm{H}-2 \mathrm{a}, \mathrm{H}-3, \mathrm{H}-5, \mathrm{H}-9$ \\
\hline & & $1.69(\beta), m$ & $2,3,5,10,20$ & $\mathrm{H}-2 \mathrm{a}, \mathrm{H}-2 \beta$ & \\
\hline \multirow[t]{2}{*}{2} & $27.0, \mathrm{CH}_{2}$ & $1.67(a), m$ & & $\mathrm{H}-1 \mathrm{a}, \mathrm{H}-1 \beta, \mathrm{H}-3$ & $\mathrm{H}-1 \mathrm{a}$ \\
\hline & & $1.61(\beta), \mathrm{m}$ & 3 & $\mathrm{H}-1 \mathrm{a}, \mathrm{H}-1 \beta, \mathrm{H}-3$ & \\
\hline 3 & $78.9, \mathrm{CH}$ & $3.26, \mathrm{dd}(11.4,4.4)$ & 18,19 & $\mathrm{H}-2 \mathrm{a}, \mathrm{H}-2 \beta$ & $\mathrm{H}-1 \mathrm{a}, \mathrm{H}-5, \mathrm{H}-19$ \\
\hline 4 & $38.7, \mathrm{C}$ & & & & \\
\hline 5 & $55.0, \mathrm{CH}$ & 0.74, dd $(11.7,1.4)$ & $1,3,4,6,7,9,10,18,19,20$ & $\mathrm{H}-6 \mathrm{a}, \mathrm{H}-6 \beta$ & $\mathrm{H}-1 \mathrm{a}, \mathrm{H}-3, \mathrm{H}-6 \mathrm{a}, \mathrm{H}-7 \mathrm{a}, \mathrm{H}-9, \mathrm{H}-19$ \\
\hline \multirow[t]{2}{*}{6} & $17.8, \mathrm{CH}_{2}$ & $1.58(a)$, brd (13.3) & & $\mathrm{H}-5, \mathrm{H}-7 \mathrm{a}, \mathrm{H}-7 \beta$ & $\mathrm{H}-5$ \\
\hline & & $1.45(\beta)$, qd $(13.4,2.9)$ & & $\mathrm{H}-5, \mathrm{H}-7 \mathrm{a}, \mathrm{H}-7 \beta$ & $\mathrm{H}-17, \mathrm{H}-20$ \\
\hline \multirow[t]{2}{*}{7} & $32.3, \mathrm{CH}_{2}$ & $1.30(a), \operatorname{td}(12.9,4.0)$ & $5,6,8,14,17$ & $H-6 a, H-6 \beta$ & $\mathrm{H}-5$ \\
\hline & & $1.52(\beta), \mathrm{dt}(12.6,2.8)$ & 5,6 & $\mathrm{H}-6 \mathrm{a}, \mathrm{H}-6 \beta$ & $\mathrm{H}-15 \mathrm{a}$ \\
\hline 8 & $40.9, \mathrm{C}$ & & & & \\
\hline 9 & $47.7, \mathrm{CH}$ & $1.65, \mathrm{~m}$ & $8,10,11,14,17,20$ & $\mathrm{H}-11 \mathrm{a}, \mathrm{H}-11 \beta$ & $H-1 a, H-5, H-11 a$ \\
\hline 10 & $36.7, \mathrm{C}$ & & & & \\
\hline \multirow[t]{2}{*}{11} & $23.0, \mathrm{CH}_{2}$ & $2.03(a), m$ & $8,9,12,13$ & $\mathrm{H}-9, \mathrm{H}-12$ & $\mathrm{H}-9$ \\
\hline & & $1.97(\beta), \mathrm{m}$ & 12,13 & $\mathrm{H}-9, \mathrm{H}-12$ & $\mathrm{H}-17, \mathrm{H}-20$ \\
\hline 12 & 128.3, $\mathrm{CH}$ & 5.65, brs & $9,14,16$ & $\mathrm{H}-11 \alpha, \mathrm{H}-11 \beta, \mathrm{H}-16$ & $\mathrm{H}-16$ \\
\hline 13 & $131.4, \mathrm{C}$ & & & & \\
\hline 14 & $100.2, \mathrm{C}$ & & & & \\
\hline \multirow[t]{2}{*}{15} & $28.5, \mathrm{CH}_{2}$ & $3.03(a), d(15.7)$ & $8,13,14,1^{\prime}, 2^{\prime}, 3^{\prime}$ & & $\mathrm{H}-7 \beta, \mathrm{H}-17$ \\
\hline & & $2.88(\beta), d(15.7)$ & $8,13,14,1^{\prime}, 2^{\prime}, 3^{\prime}$ & & $\mathrm{H}-16, \mathrm{H}-17$ \\
\hline 16 & $18.4, \mathrm{CH}_{3}$ & $1.63, \mathrm{brs}$ & $12,13,14$ & $\mathrm{H}-12$ & $\mathrm{H}-12, \mathrm{H}-15 \beta$ \\
\hline 17 & $16.2, \mathrm{CH}_{3}$ & $0.90, \mathrm{~s}$ & $7,8,9,14$ & & $\mathrm{H}-6 \beta, \mathrm{H}-11 \beta, \mathrm{H}-15 \mathrm{a}, \mathrm{H}-15 \beta$ \\
\hline 18 & $15.6, \mathrm{CH}_{3}$ & $0.80, \mathrm{~s}$ & $3,4,5,19$ & & \\
\hline 19 & $28.1, \mathrm{CH}_{3}$ & $0.98, \mathrm{~s}$ & $3,4,5,18$ & & $\mathrm{H}-3, \mathrm{H}-5$ \\
\hline 20 & $15.7, \mathrm{CH}_{3}$ & $0.95, \mathrm{~s}$ & $1,5,9,10$ & & $\mathrm{H}-6 \beta, \mathrm{H}-11 \beta$ \\
\hline $1^{\prime}$ & $162.7, \mathrm{C}$ & & & & \\
\hline $2^{\prime}$ & $99.7, \mathrm{C}$ & & & & \\
\hline $3^{\prime}$ & $171.6, \mathrm{C}$ & & & & \\
\hline $4^{\prime}$ & 103.3, C & & & & \\
\hline $5^{\prime}$ & $160.5, \mathrm{C}$ & & & & \\
\hline $6^{\prime}$ & $17.2, \mathrm{CH}_{3}$ & $2.23, \mathrm{~s}$ & $4^{\prime}, 5^{\prime}$ & & $\mathrm{H}-7^{\prime}$ \\
\hline $7^{\prime}$ & 9.7, $\mathrm{CH}_{3}$ & $1.95, \mathrm{~s}$ & $3^{\prime}, 4^{\prime}, 5^{\prime}$ & & $\mathrm{H}-6^{\prime}$ \\
\hline
\end{tabular}

${ }^{1} \mathrm{H}$ NMR: $600 \mathrm{MHz},{ }^{13} \mathrm{C}$ NMR: $150 \mathrm{MHz}$ (in $\mathrm{CDCl}_{3}$ ) 
Table S7. NMR data for brevione R (6).

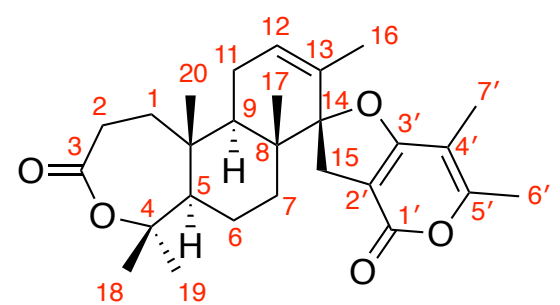

\begin{tabular}{|c|c|c|c|c|c|}
\hline position & $\delta_{c}$, type & $\delta_{\mathrm{H}}$, mult. $(J$ in $\mathrm{Hz})$ & HMBC correlation & COSY correlation & NOESY correlation \\
\hline \multirow[t]{2}{*}{1} & $37.7 \mathrm{CH}_{2}$ & $1.59(a), m$ & $3,5,9,20$ & $\mathrm{H}-2$ & \\
\hline & & $1.83(\beta)$, ddd $(14.0,9.9,3.6)$ & $2,3,5,10,20$ & $\mathrm{H}-2$ & $\mathrm{H}-20$ \\
\hline 2 & $31.9, \mathrm{CH}_{2}$ & $2.63, \mathrm{~m}$ & $1,3,10$ & $\mathrm{H}-1 \mathrm{a}, \mathrm{H}-1 \beta$ & \\
\hline 3 & $174.9, \mathrm{C}$ & & & & \\
\hline 4 & $85.9, \mathrm{C}$ & & & & \\
\hline 5 & $54.4, \mathrm{CH}$ & 1.66, dd $(11.7,2.2)$ & $4,6,10,18,20$ & $\mathrm{H}-6 \mathrm{a}, \mathrm{H}-6 \beta$ & $\mathrm{H}-6 \mathrm{a}, \mathrm{H}-7 \mathrm{a}, \mathrm{H}-19$ \\
\hline \multirow[t]{2}{*}{6} & $22.1, \mathrm{CH}_{2}$ & $1.55(a), m$ & 5,10 & $\mathrm{H}-5, \mathrm{H}-7 \mathrm{a}, \mathrm{H}-7 \beta$ & $\mathrm{H}-5$ \\
\hline & & $1.60(\beta), m$ & 7,10 & $\mathrm{H}-5, \mathrm{H}-7 \mathrm{a}, \mathrm{H}-7 \beta$ & $\mathrm{H}-20$ \\
\hline \multirow[t]{2}{*}{7} & $31.6, \mathrm{CH}_{2}$ & $1.35(a), \operatorname{td}(12.9,4.0)$ & 8,17 & $H-6 a, H-6 \beta$ & $\mathrm{H}-5, \mathrm{H}-9$ \\
\hline & & $1.52(\beta), \mathrm{m}$ & $5,8,9$ & $\mathrm{H}-6 \mathrm{a}, \mathrm{H}-6 \beta$ & $\mathrm{H}-15 \mathrm{a}, \mathrm{H}-17$ \\
\hline 8 & $40.9, \mathrm{C}$ & & & & \\
\hline 9 & $47.5, \mathrm{CH}$ & $1.75, \mathrm{dd}(11.5,5.4)$ & $1,5,8,10,11,17,20$ & $\mathrm{H}-11 a, \mathrm{H}-11 \beta$ & $\mathrm{H}-7 \mathrm{a}, \mathrm{H}-11 \mathrm{a}$ \\
\hline 10 & $39.5 \mathrm{C}$ & & & & \\
\hline \multirow[t]{2}{*}{11} & $23.4, \mathrm{CH}_{2}$ & $2.07(a), m$ & $8,9,12,13$ & $\mathrm{H}-9, \mathrm{H}-12$ & $\mathrm{H}-9$ \\
\hline & & $2.04(\beta), m$ & 12,13 & $\mathrm{H}-9, \mathrm{H}-12$ & $\mathrm{H}-17, \mathrm{H}-20$ \\
\hline 12 & $127.8, \mathrm{CH}$ & $5.65, \mathrm{dd}(2.7,1.6)$ & $9,14,16$ & $\mathrm{H}-11 a, \mathrm{H}-11 \beta, \mathrm{H}-16$ & $\mathrm{H}-16$ \\
\hline 13 & $131.5, \mathrm{C}$ & & & & \\
\hline 14 & $99.4, \mathrm{C}$ & & & & \\
\hline \multirow[t]{2}{*}{15} & $28.5, \mathrm{CH}_{2}$ & $3.00(a), d(15.8)$ & $8,13,14,1^{\prime}, 2^{\prime}, 3^{\prime}$ & & $\mathrm{H}-7 \beta, \mathrm{H}-17$ \\
\hline & & $2.88(\beta), d(15.8)$ & $8,13,14,1^{\prime}, 2^{\prime}, 3^{\prime}$ & & $\mathrm{H}-16$ \\
\hline 16 & $18.2, \mathrm{CH}_{3}$ & $1.62, \mathrm{brs}$ & $12,13,14$ & $\mathrm{H}-12$ & $\mathrm{H}-12, \mathrm{H}-15 \beta$ \\
\hline 17 & $15.7, \mathrm{CH}_{3}$ & $0.95, \mathrm{~s}$ & $7,8,9,14$ & & $H-7 \beta, H-11 \beta, H-15 a$ \\
\hline 18 & $25.7, \mathrm{CH}_{3}$ & $1.43, \mathrm{~s}$ & $4,5,19$ & & $\mathrm{H}-20$ \\
\hline 19 & $32.4, \mathrm{CH}_{3}$ & $1.46, \mathrm{~s}$ & $4,5,18$ & & $\mathrm{H}-5$ \\
\hline 20 & $17.2, \mathrm{CH}_{3}$ & $1.16, \mathrm{~s}$ & $1,5,9,10$ & & $\mathrm{H}-1 \beta, \mathrm{H}-6 \beta, \mathrm{H}-11 \beta, \mathrm{H}-18$ \\
\hline $1^{\prime}$ & $161.9, \mathrm{C}$ & & & & \\
\hline $2^{\prime}$ & $99.4, \mathrm{C}$ & & & & \\
\hline $3^{\prime}$ & $171.0, \mathrm{C}$ & & & & \\
\hline $4^{\prime}$ & $102.8, \mathrm{C}$ & & & & \\
\hline $5^{\prime}$ & $160.5, \mathrm{C}$ & & & & \\
\hline $6^{\prime}$ & $17.2, \mathrm{CH}_{3}$ & $2.21, \mathrm{~s}$ & $4^{\prime}, 5^{\prime}$ & & $\mathrm{H}-7^{\prime}$ \\
\hline $7^{\prime}$ & $9.7, \mathrm{CH}_{3}$ & $1.93, \mathrm{~s}$ & $3^{\prime}, 4^{\prime}, 5^{\prime}$ & & $\mathrm{H}-6^{\prime}$ \\
\hline
\end{tabular}

${ }^{1} \mathrm{H}$ NMR: $600 \mathrm{MHz},{ }^{13} \mathrm{C}$ NMR: $150 \mathrm{MHz}\left(\right.$ in $\mathrm{CDCl}_{3}$ ) 
Table S8. NMR data for brevione S (7).

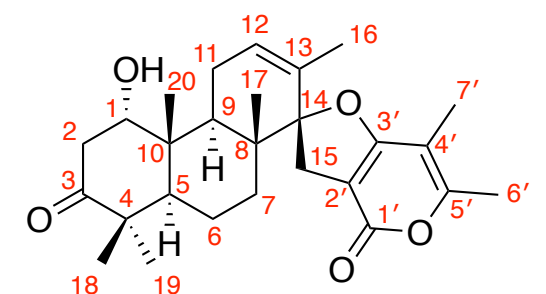

\begin{tabular}{|c|c|c|c|c|c|}
\hline position & $\delta_{c}$, type & $\delta_{\mathrm{H}}$, mult. $(J$ in Hz) & HMBC correlation & COSY correlation & NOESY correlation \\
\hline 1 & $73.2, \mathrm{CH}$ & $4.00, \mathrm{~m}$ & 3,5 & $\mathrm{H}-2 \mathrm{a}, \mathrm{H}-2 \beta, 1-\mathrm{OH}$ & $\mathrm{H}-2 \mathrm{a}, \mathrm{H}-2 \beta, \mathrm{H}-20$ \\
\hline \multirow[t]{2}{*}{2} & $42.8, \mathrm{CH}_{2}$ & $2.39(a), d d(16.2,3.2)$ & $1,3,4,10$ & $\mathrm{H}-1$ & $\mathrm{H}-1,1-\mathrm{OH}$ \\
\hline & & $3.05(\beta), \mathrm{dd}(16.2,3.6)$ & 1,3 & $\mathrm{H}-1$ & $\mathrm{H}-1, \mathrm{H}-20$ \\
\hline 3 & $215.3 \mathrm{C}$ & & & & \\
\hline 4 & $47.5, \mathrm{C}$ & & & & \\
\hline 5 & $47.4 \mathrm{CH}$ & 1.76, dd $(12.0,2.1)$ & $10,18,19,20$ & $\mathrm{H}-6 \mathrm{a}, \mathrm{H}-6 \beta$ & $\mathrm{H}-7 \mathrm{a}, \mathrm{H}-9, \mathrm{H}-19$ \\
\hline \multirow[t]{2}{*}{6} & $18.4, \mathrm{CH}_{2}$ & $1.55(\mathrm{a}), \mathrm{m}$ & 5 & $\mathrm{H}-5, \mathrm{H}-7 \mathrm{a}, \mathrm{H}-7 \beta$ & \\
\hline & & $1.62(\beta), m$ & 8 & $\mathrm{H}-5, \mathrm{H}-7 \mathrm{a}, \mathrm{H}-7 \beta$ & $\mathrm{H}-17, \mathrm{H}-20$ \\
\hline \multirow[t]{2}{*}{7} & $31.5, \mathrm{CH}_{2}$ & $1.33(a), \operatorname{td}(12.7,4.2)$ & $5,6,8,14,17$ & $\mathrm{H}-6 \mathrm{a}, \mathrm{H}-6 \beta$ & $\mathrm{H}-5$ \\
\hline & & $1.54(\beta), \mathrm{m}$ & $5,6,9$ & $\mathrm{H}-6 \mathrm{a}, \mathrm{H}-6 \beta$ & \\
\hline 8 & $40.8, \mathrm{C}$ & & & & \\
\hline 9 & $38.2, \mathrm{CH}$ & 2.48, dd $(10.2,6.9)$ & $1,8,10,14,17,20$ & $\mathrm{H}-11$ & $\mathrm{H}-5$ \\
\hline 10 & $40.8, \mathrm{C}$ & & & & \\
\hline 11 & $22.7, \mathrm{CH}_{2}$ & $2.05, \mathrm{~m}$ & $9,12,13$ & $\mathrm{H}-9, \mathrm{H}-12$ & \\
\hline 12 & 127.6, $\mathrm{CH}$ & 5.65 , brs & $9,11,14,16$ & $\mathrm{H}-11, \mathrm{H}-16$ & $\mathrm{H}-16$ \\
\hline 13 & $131.9, \mathrm{C}$ & & & & \\
\hline 14 & $99.7, \mathrm{C}$ & & & & \\
\hline \multirow[t]{2}{*}{15} & 28.7, $\mathrm{CH}_{2}$ & $3.04(a), d(15.7)$ & $8,13,14,1^{\prime}, 2^{\prime}, 3^{\prime}$ & & $\mathrm{H}-17$ \\
\hline & & $2.91(\beta), d(15.7)$ & $8,13,14,1^{\prime}, 2^{\prime}, 3^{\prime}$ & & $\mathrm{H}-16$ \\
\hline 16 & $18.5, \mathrm{CH}_{3}$ & 1.64, brs & $12,13,14$ & $\mathrm{H}-12$ & $\mathrm{H}-12, \mathrm{H}-15 \beta$ \\
\hline 17 & $16.3, \mathrm{CH}_{3}$ & $0.97, \mathrm{~s}$ & $7,8,9,14$ & & $\mathrm{H}-6 \beta, \mathrm{H}-15 \mathrm{a}$ \\
\hline 18 & $21.8, \mathrm{CH}_{3}$ & $1.06, \mathrm{~s}$ & $3,4,5,19$ & & \\
\hline 19 & $25.2, \mathrm{CH}_{3}$ & $1.08, \mathrm{~s}$ & $3,4,5,18$ & & $H-5$ \\
\hline 20 & $15.7, \mathrm{CH}_{3}$ & $1.15, \mathrm{~s}$ & $1,5,9,10$ & & $H-1, H-2 \beta, H-6 \beta$ \\
\hline $1^{\prime}$ & 162.0, C & & & & \\
\hline $2^{\prime}$ & $99.4, \mathrm{C}$ & & & & \\
\hline $3^{\prime}$ & $171.1, \mathrm{C}$ & & & & \\
\hline $4^{\prime}$ & $102.9, \mathrm{C}$ & & & & \\
\hline $5^{\prime}$ & $160.4, \mathrm{C}$ & & & & \\
\hline $6^{\prime}$ & $17.1, \mathrm{CH}_{3}$ & $2.20, \mathrm{~s}$ & $4^{\prime}, 5^{\prime}$ & & $\mathrm{H}-7^{\prime}$ \\
\hline $7^{\prime}$ & $9.6, \mathrm{CH}_{3}$ & $1.90, \mathrm{~s}$ & $3^{\prime}, 4^{\prime}, 5^{\prime}$ & & $H-6^{\prime}$ \\
\hline $1-\mathrm{OH}$ & & $1.88 \mathrm{~d}(2.3)$ & & $\mathrm{H}-1$ & $\mathrm{H}-2 \mathrm{a}$ \\
\hline
\end{tabular}

${ }^{1} \mathrm{H}$ NMR: $600 \mathrm{MHz},{ }^{13} \mathrm{C}$ NMR: $150 \mathrm{MHz}$ (in $\mathrm{CDCl}_{3}$ ) 
Table S9. NMR data for brevione T (8).

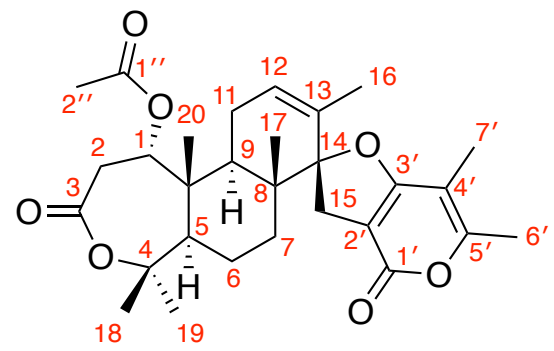

\begin{tabular}{|c|c|c|c|c|c|}
\hline position & бc, type & $\delta_{\mathrm{H}}$, mult. $(\mathrm{J}$ in $\mathrm{Hz})$ & HMBC correlation & COSY correlation & NOESY correlation \\
\hline 1 & $70.5, \mathrm{CH}$ & $4.91, \mathrm{dd}(6.4,1.6)$ & $2,3,5,9,10$ & $\mathrm{H}-2 \mathrm{a}, \mathrm{H}-2 \beta$ & $\mathrm{H}-2 \mathrm{a}, \mathrm{H}-2 \beta, \mathrm{H}-20$ \\
\hline \multirow[t]{2}{*}{2} & $35.3, \mathrm{CH}_{2}$ & $3.08(a)$, dd $(15.7,6.5)$ & $1,3,10$ & $\mathrm{H}-1$ & $\mathrm{H}-1$ \\
\hline & & $3.11(\beta), \mathrm{dd}(15.7,1.6)$ & $1,3,10$ & $\mathrm{H}-1$ & $\mathrm{H}-1, \mathrm{H}-18, \mathrm{H}-20$ \\
\hline 3 & $170.4, \mathrm{C}$ & & & & \\
\hline 4 & $85.8, \mathrm{C}$ & & & & \\
\hline 5 & $50.7, \mathrm{CH}$ & $1.94, \mathrm{~m}$ & $4,6,10,18,20$ & $H-6 a, H-6 \beta$ & $\mathrm{H}-6 \mathrm{a}, \mathrm{H}-7 \mathrm{a}, \mathrm{H}-19$ \\
\hline \multirow[t]{2}{*}{6} & $21.0, \mathrm{CH}_{2}$ & $1.71(\mathrm{a}), \mathrm{m}$ & & $\mathrm{H}-5, \mathrm{H}-7 \mathrm{a}, \mathrm{H}-7 \beta$ & $\mathrm{H}-5, \mathrm{H}-7 \mathrm{a}$ \\
\hline & & $1.57(\beta), \mathrm{m}$ & & $\mathrm{H}-5, \mathrm{H}-7 \mathrm{a}, \mathrm{H}-7 \beta$ & $\mathrm{H}-20$ \\
\hline \multirow[t]{2}{*}{7} & $31.7, \mathrm{CH}_{2}$ & $1.34(a), \operatorname{td}(13.7,4.0)$ & $5,6,8,14,17$ & $\mathrm{H}-6 \mathrm{a}, \mathrm{H}-6 \beta$ & $\mathrm{H}-5, \mathrm{H}-6 \mathrm{a}, \mathrm{H}-9$ \\
\hline & & $1.54(\beta), m$ & 5 & $\mathrm{H}-6 \mathrm{a}, \mathrm{H}-6 \beta$ & $\mathrm{H}-15 a, \mathrm{H}-17$ \\
\hline 8 & $40.7, \mathrm{C}$ & & & & \\
\hline 9 & $39.0, \mathrm{CH}$ & $2.33, \mathrm{dd}(10.8,6.1)$ & $8,10,11,14,17,20$ & $\mathrm{H}-11$ & $\mathrm{H}-7 \mathrm{a}$ \\
\hline 10 & $43.7, \mathrm{C}$ & & & & \\
\hline 11 & $22.9, \mathrm{CH}_{2}$ & $1.95, \mathrm{~m}$ & & $\mathrm{H}-9, \mathrm{H}-12$ & \\
\hline 12 & $127.5, \mathrm{CH}$ & $5.58, \mathrm{brs}$ & $9,14,16$ & $\mathrm{H}-11, \mathrm{H}-16$ & $\mathrm{H}-16$ \\
\hline 13 & $131.4, \mathrm{C}$ & & & & \\
\hline 14 & $99.5, \mathrm{C}$ & & & & \\
\hline \multirow[t]{2}{*}{15} & $28.4, \mathrm{CH}_{2}$ & $2.99(a), d(15.3)$ & $8,13,14,1^{\prime}, 2^{\prime}, 3^{\prime}$ & & $\mathrm{H}-7 \beta, \mathrm{H}-17$ \\
\hline & & $2.92(\beta), d(15.3)$ & $8,13,14,1^{\prime}, 2^{\prime}, 3^{\prime}$ & & $\mathrm{H}-16$ \\
\hline 16 & 18.6, $\mathrm{CH}_{3}$ & 1.63, brd (1.5) & $12,13,14$ & $\mathrm{H}-12$ & $\mathrm{H}-12, \mathrm{H}-15 \beta$ \\
\hline 17 & $16.2, \mathrm{CH}_{3}$ & $0.93, \mathrm{~s}$ & $7,8,9,14$ & & $\mathrm{H}-7 \beta, \mathrm{H}-15 \mathrm{a}, \mathrm{H}-20$ \\
\hline 18 & $24.0, \mathrm{CH}_{3}$ & $1.51, \mathrm{~s}$ & $4,5,19$ & & $\mathrm{H}-2 \beta, \mathrm{H}-20$ \\
\hline 19 & $34.6, \mathrm{CH}_{3}$ & $1.49, \mathrm{~s}$ & $3,4,5,18$ & & $\mathrm{H}-5$ \\
\hline 20 & $15.0, \mathrm{CH}_{3}$ & $1.20, \mathrm{~s}$ & $1,5,9,10$ & & $\mathrm{H}-1, \mathrm{H}-2 \beta, \mathrm{H}-6 \beta, \mathrm{H}-17, \mathrm{H}-18$ \\
\hline $1^{\prime}$ & 161.9, C & & & & \\
\hline $2^{\prime}$ & $99.2, \mathrm{C}$ & & & & \\
\hline $3^{\prime}$ & $170.9, \mathrm{C}$ & & & & \\
\hline $4^{\prime}$ & 102.6, C & & & & \\
\hline $5^{\prime}$ & 160.6, C & & & & \\
\hline $6^{\prime}$ & $17.2, \mathrm{CH}_{3}$ & $2.22, \mathrm{~s}$ & $4^{\prime}, 5^{\prime}$ & & $\mathrm{H}-7^{\prime}$ \\
\hline $7^{\prime}$ & $9.3, \mathrm{CH}_{3}$ & $1.89, \mathrm{~s}$ & $3^{\prime}, 4^{\prime}, 5^{\prime}$ & & $\mathrm{H}-6^{\prime}$ \\
\hline $1^{\prime \prime}$ & $169.8, \mathrm{C}$ & & & & \\
\hline $2^{\prime \prime}$ & $20.7, \mathrm{CH}_{3}$ & $2.07, \mathrm{~s}$ & $1^{\prime \prime}$ & & \\
\hline
\end{tabular}

${ }^{1} \mathrm{H}$ NMR: $600 \mathrm{MHz},{ }^{13} \mathrm{C}$ NMR: $150 \mathrm{MHz}$ (in $\mathrm{CDCl}_{3}$ ) 
Table S10. NMR data for setosusin (1) obtained from the isotope incorporation experiments.

\begin{tabular}{|c|c|c|c|c|c|}
\hline position & $\begin{array}{c}\delta c \\
(\mathrm{ppm})\end{array}$ & $\begin{array}{c}{ }^{1} \mathrm{Jcc} \text { from }\left[1,2-{ }^{13} \mathrm{C}_{2}\right] \text {-acetate }(\mathrm{Hz}) \\
\text { / Coupled carbon }\end{array}$ & $\begin{array}{c}\text { Enrichment by } \\
{\left[1-{ }^{13} \mathrm{C},{ }^{18} \mathrm{O}_{2}\right] \text { acetate }} \\
(\bullet: \text { enriched })\end{array}$ & $\begin{array}{c}{ }^{18} \mathrm{O} \text { isotope shift from } \\
{\left[1-{ }^{13} \mathrm{C},{ }^{18} \mathrm{O}_{2}\right] \text { acetate }\left(\Delta_{\delta}, \mathrm{ppm}\right)}\end{array}$ & $\begin{array}{l}{ }^{18} \mathrm{O} \text { isotope shift from } \\
{ }^{18} \mathrm{O}_{2}(\Delta \delta, \mathrm{ppm})\end{array}$ \\
\hline 1 & 70.3 & - & - & - & - \\
\hline 2 & 35.3 & 52.4 / C-3 & $\bullet$ & - & - \\
\hline 3 & 170.5 & 52.4 / C-2 & - & - & - \\
\hline 4 & 85.6 & $38.1 / C-17$ & $\bullet$ & - & - \\
\hline 5 & 50.3 & 33.4 / C-6 & - & - & - \\
\hline 6 & 21.1 & 33.4 / C-5 & $\bullet$ & - & - \\
\hline 7 & 31.7 & - & - & - & - \\
\hline 8 & 41.0 & 35.8 / C-18 & $\bullet$ & - & - \\
\hline 9 & 39.1 & $34.6 / C-11$ & - & - & - \\
\hline 10 & 43.9 & 35.8 / C-19 & $\bullet$ & - & - \\
\hline 11 & 22.9 & 34.6 / C-9 & $\bullet$ & - & - \\
\hline 12 & 129.9 & - & - & - & - \\
\hline 13 & 130.5 & 45.3 / C-20 & $\bullet$ & - & - \\
\hline 14 & 90.2 & $35.8 / C-15$ & - & - & - \\
\hline 15 & 32.6 & $35.8 / C-14$ & $\bullet$ & - & - \\
\hline 16 & 34.5 & - & - & - & - \\
\hline 17 & 24.0 & $38.1 / C-4$ & - & - & - \\
\hline 18 & 16.7 & 35.8 / C-8 & - & - & - \\
\hline 19 & 15.1 & $35.8 / C-10$ & - & - & - \\
\hline 20 & 19.6 & $45.3 / C-13$ & - & - & - \\
\hline $1^{\prime}$ & 169.0 & $54.8 / C-2^{\prime}$ & $\bullet$ & -0.034 & - \\
\hline $2^{\prime}$ & 87.0 & $54.8 / C-1^{\prime}$ & - & - & -0.029 \\
\hline $3^{\prime}$ & 199.7 & $56.0 / C-4^{\prime}$ & $\bullet$ & -0.021 & - \\
\hline $4^{\prime}$ & 110.6 & $56.0 / C-3^{\prime}$ & - & - & - \\
\hline $5^{\prime}$ & 185.7 & $50.1 / C-6^{\prime}$ & $\bullet$ & - & -0.020 \\
\hline $6^{\prime}$ & 15.0 & $50.1 / C-5^{\prime}$ & - & - & - \\
\hline $7^{\prime}$ & 5.8 & - & - & - & - \\
\hline $1^{\prime \prime}$ & 170.4 & $59.6 / \mathrm{C}-2^{\prime \prime}$ & $\bullet$ & -0.042 & - \\
\hline $2^{\prime \prime}$ & 21.0 & $59.6 / \mathrm{C}-1^{\prime \prime}$ & - & - & - \\
\hline
\end{tabular}


(A)

$$
\text { (1) }
$$

(B)

$$
\text { (1) acetyl-CoA }
$$

Figure S1. (A) Biosynthetic pathway of decaturin $\mathrm{D}^{20}$ and (B) proposed biosynthetic route leading to brevione B (2).

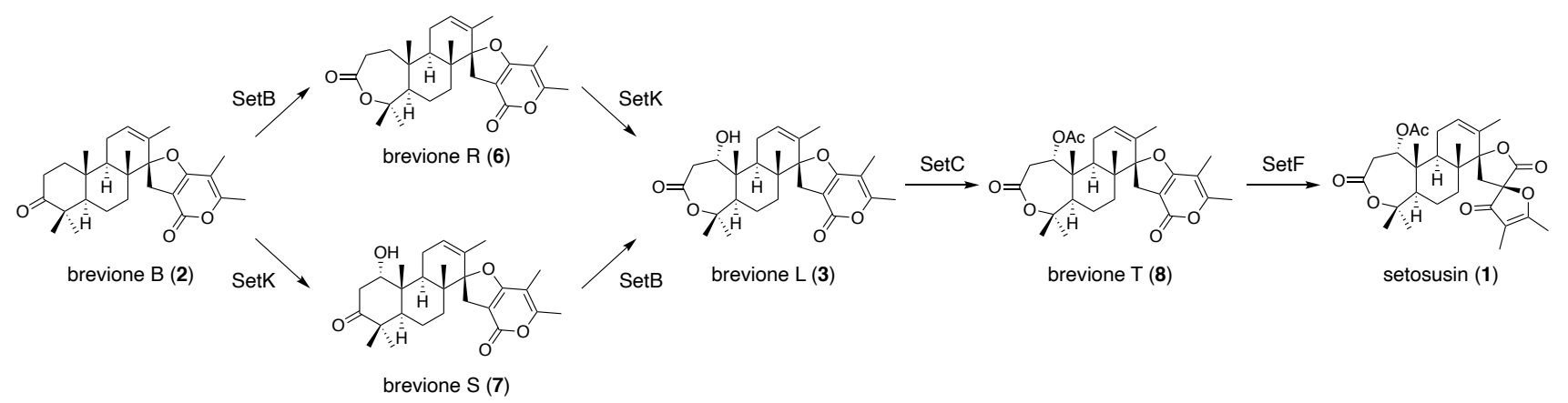

Figure S2. Late-stage biosynthesis of setosusin (1) established in this study. 


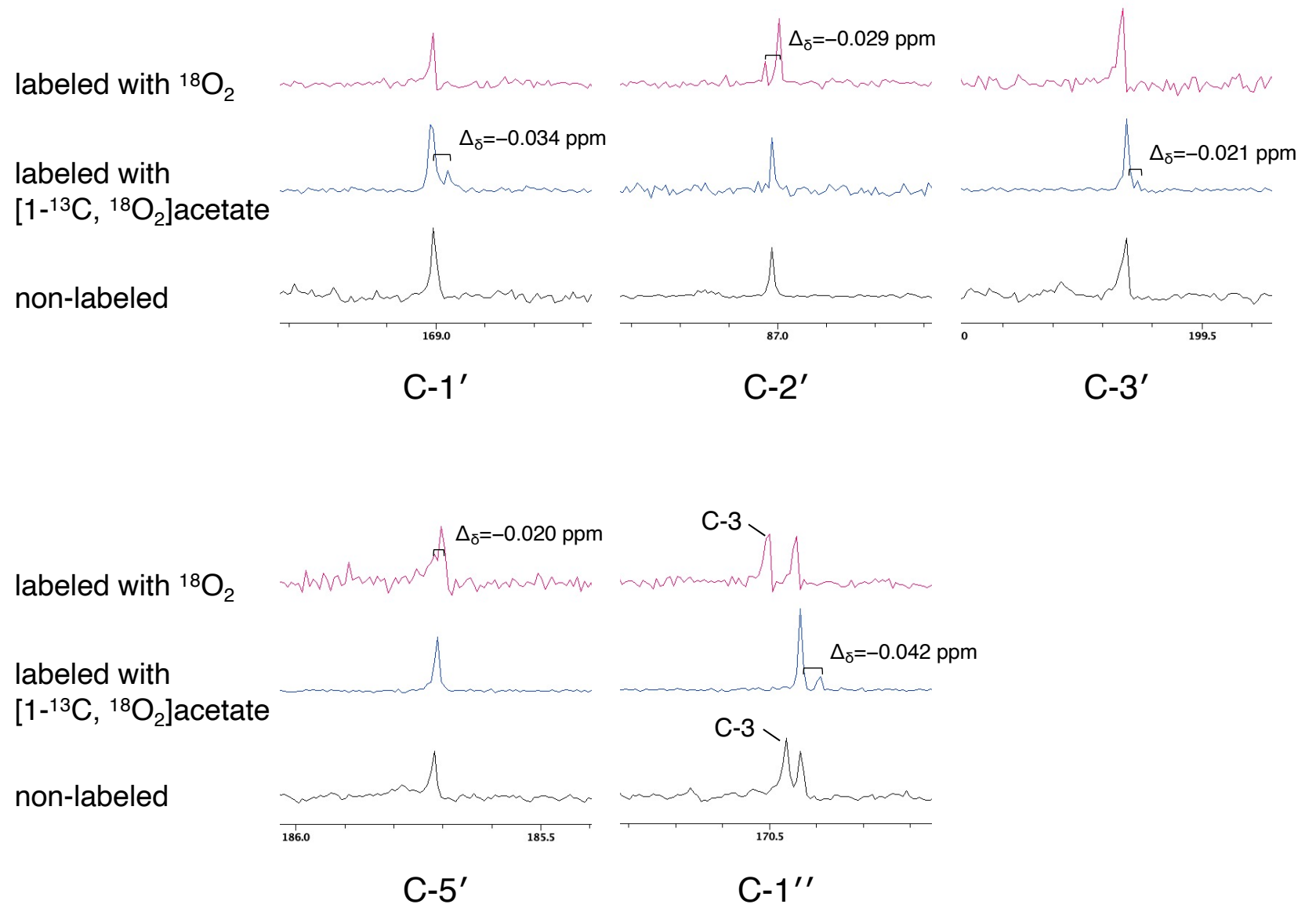

Figure S3. Partial ${ }^{13} \mathrm{C}$ NMR spectra of setosusin (1) labeled with sodium $\left[1-{ }^{13} \mathrm{C},{ }^{18} \mathrm{O}_{2}\right]$ acetate or ${ }^{18} \mathrm{O}_{2}$. See Figure S45 and Figure S46 for the complete spectra. 
(A)

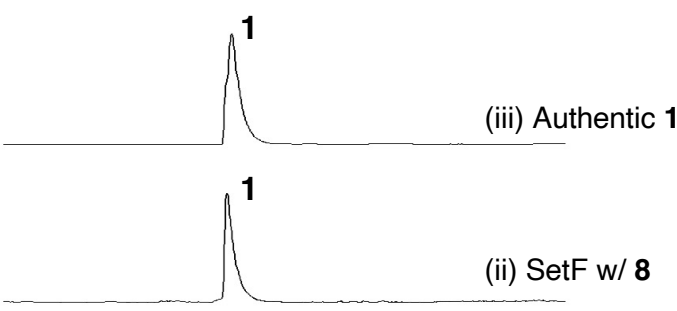

(i) Control w/ 8

$\begin{array}{ccc}10 & 15 \\ & \text { Time (min) } & \end{array}$

(B) $[\mathrm{M}+\mathrm{Na}]^{+}$

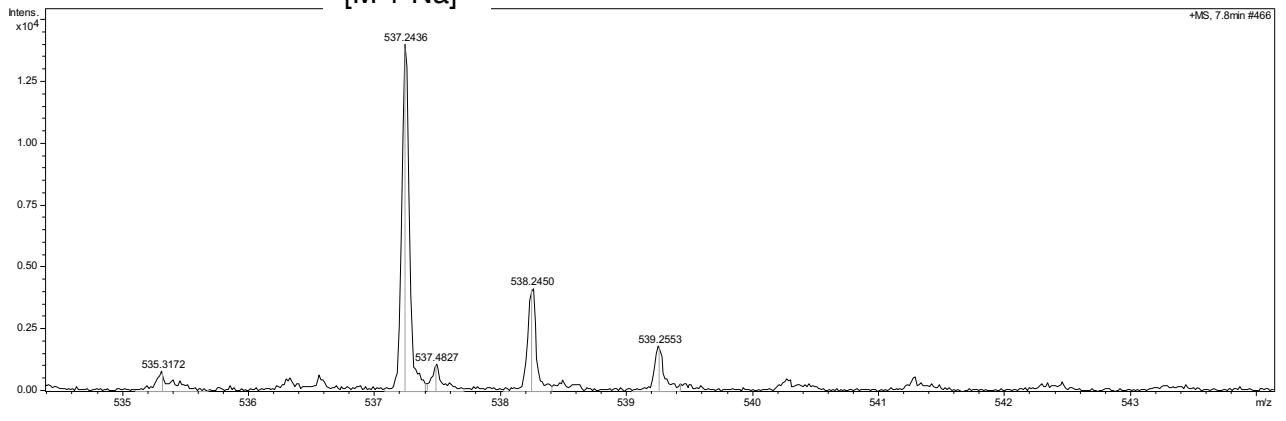

(C) $+2$

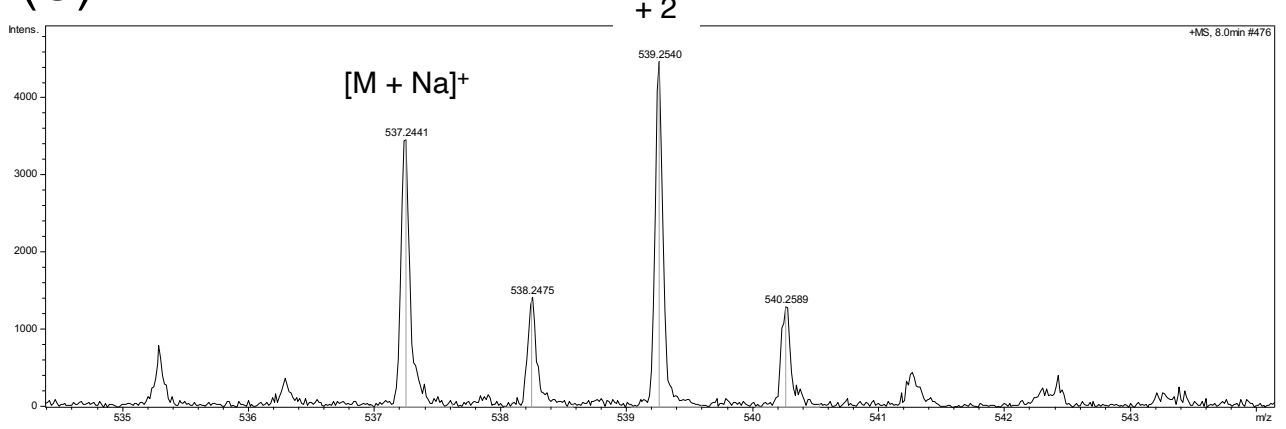

(D)

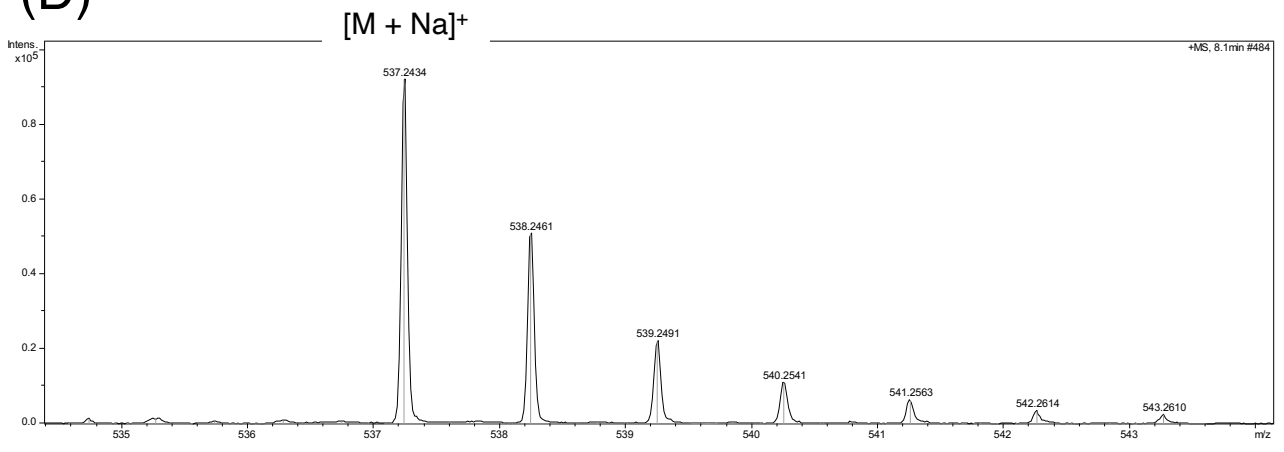

Figure S4. (A) LC-MS chromatograms of the products from in vitro enzymatic reactions with crude enzyme extract containing SetF using brevione T (8) as a substrate. Crude enzyme extract from $A$. oryzae NSAR1 was used as a negative control (trace i). The chromatograms were extracted at $m / z 537.246$ (for [M $+\mathrm{Na}]^{+}$of $\mathbf{1}$ ). (BD) Mass spectra of setosusin (1) obtained from the enzymatic reactions in (B) $\mathrm{H}_{2}{ }^{16} \mathrm{O}$ and (C) $\mathrm{H}_{2}{ }^{18} \mathrm{O}$ and (D) 1 incubated in $\mathrm{H}_{2}{ }^{18} \mathrm{O}$. 
(A)<smiles>CC1=CCC2[C@@H](C)CCC[C@]2(C)[C@]12CC1=C(C)C(C)=C(C)O[C@H]1O2</smiles>

(B)

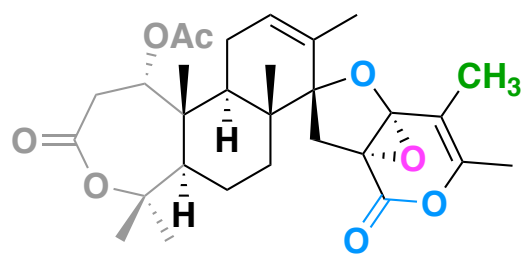

Figure S5. (A) Actual structure of IM0 used for the calculation. (B) Structure of the expected product of the SetF-catalyzed oxidative reaction. The substructure omitted for the calculation is shown in grey.

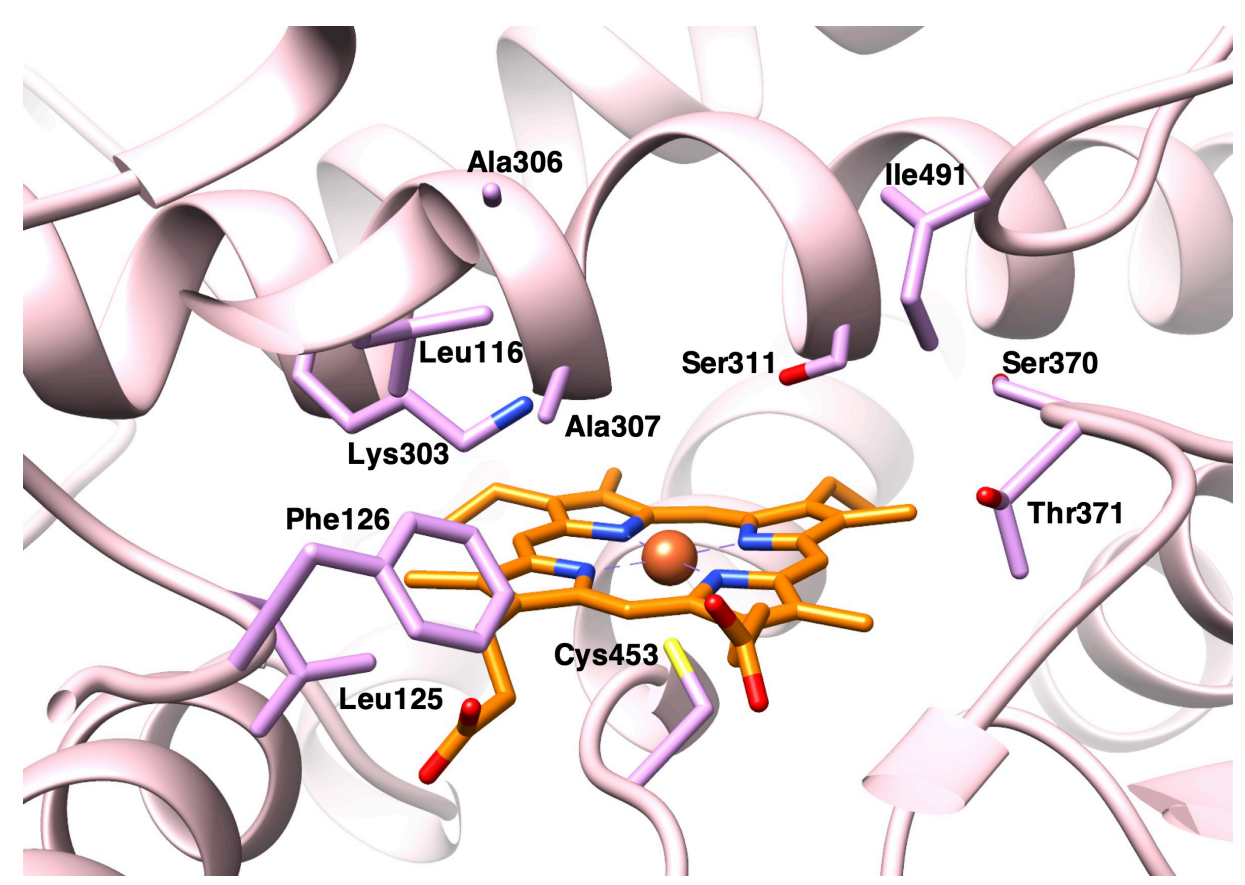

Figure S6. Predicted substrate binding site of SetF. The protein model was generated using Phyre $2,{ }^{21}$ and the heme cofactor was modelled using COFACTOR. ${ }^{22}$ 


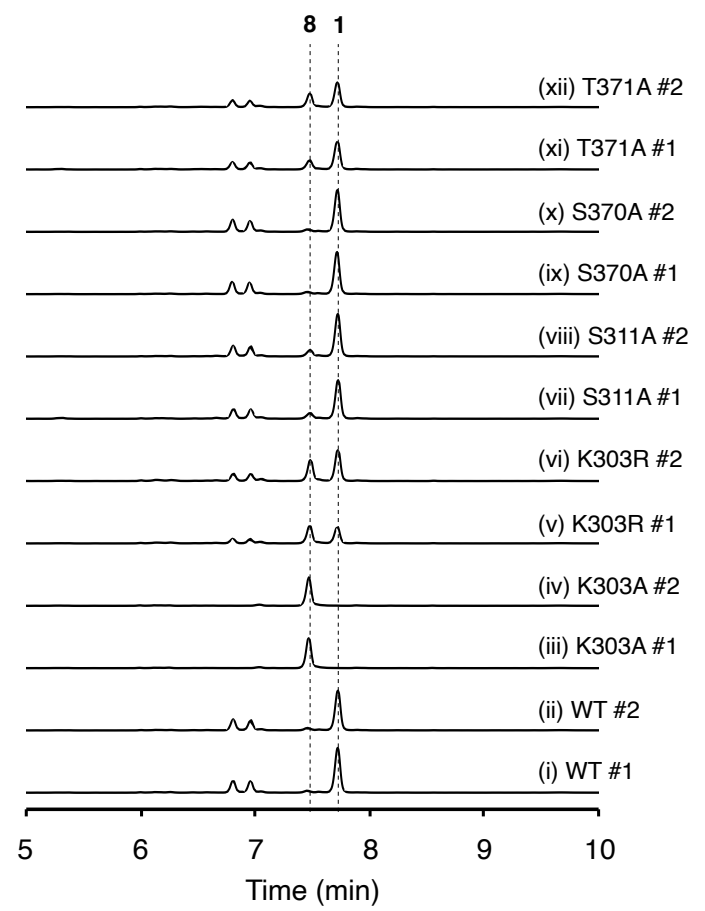

Figure S7. HPLC profiles of the bioconversion experiment with A. oryzae transformants expressing setF and its variants. The chromatograms were monitored at $280 \mathrm{~nm}$. Two individual transformants were used for each variant. WT: wild type.

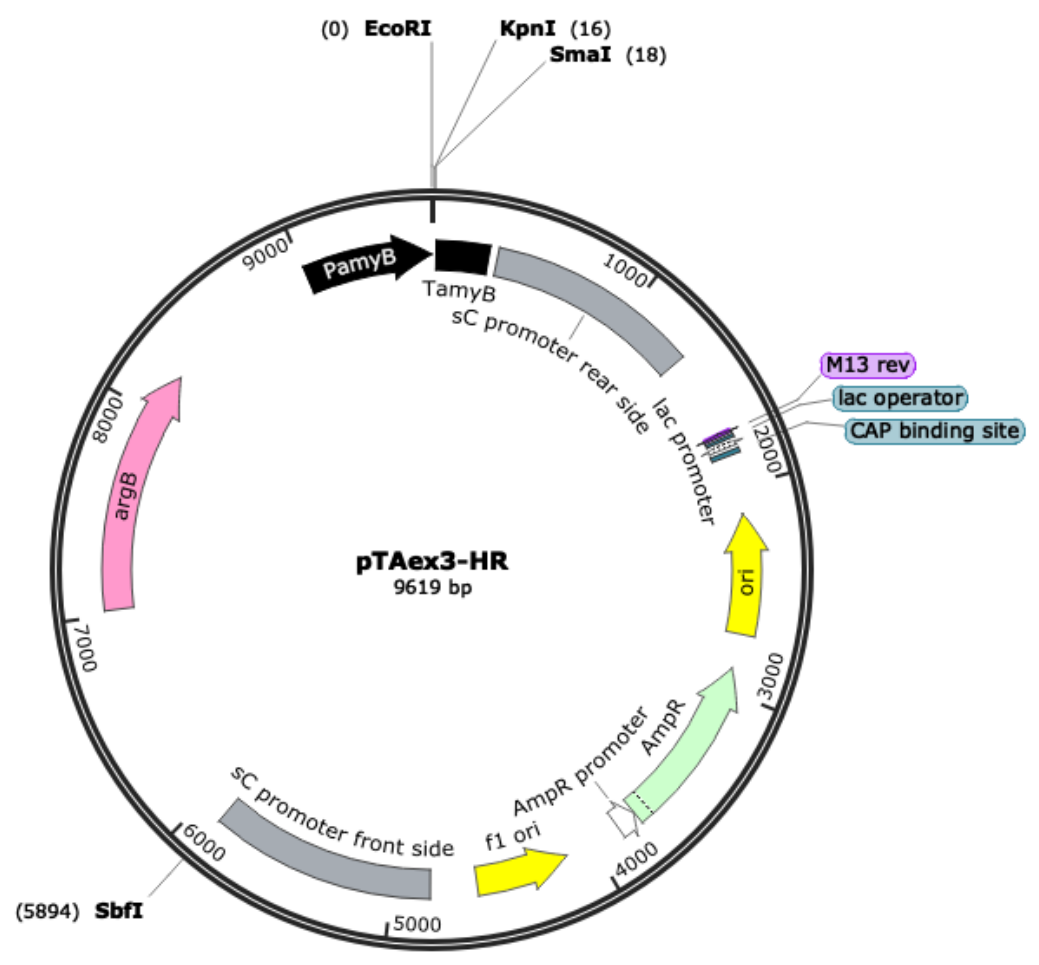

Figure S8. Vector map of pTAex3-HR. 


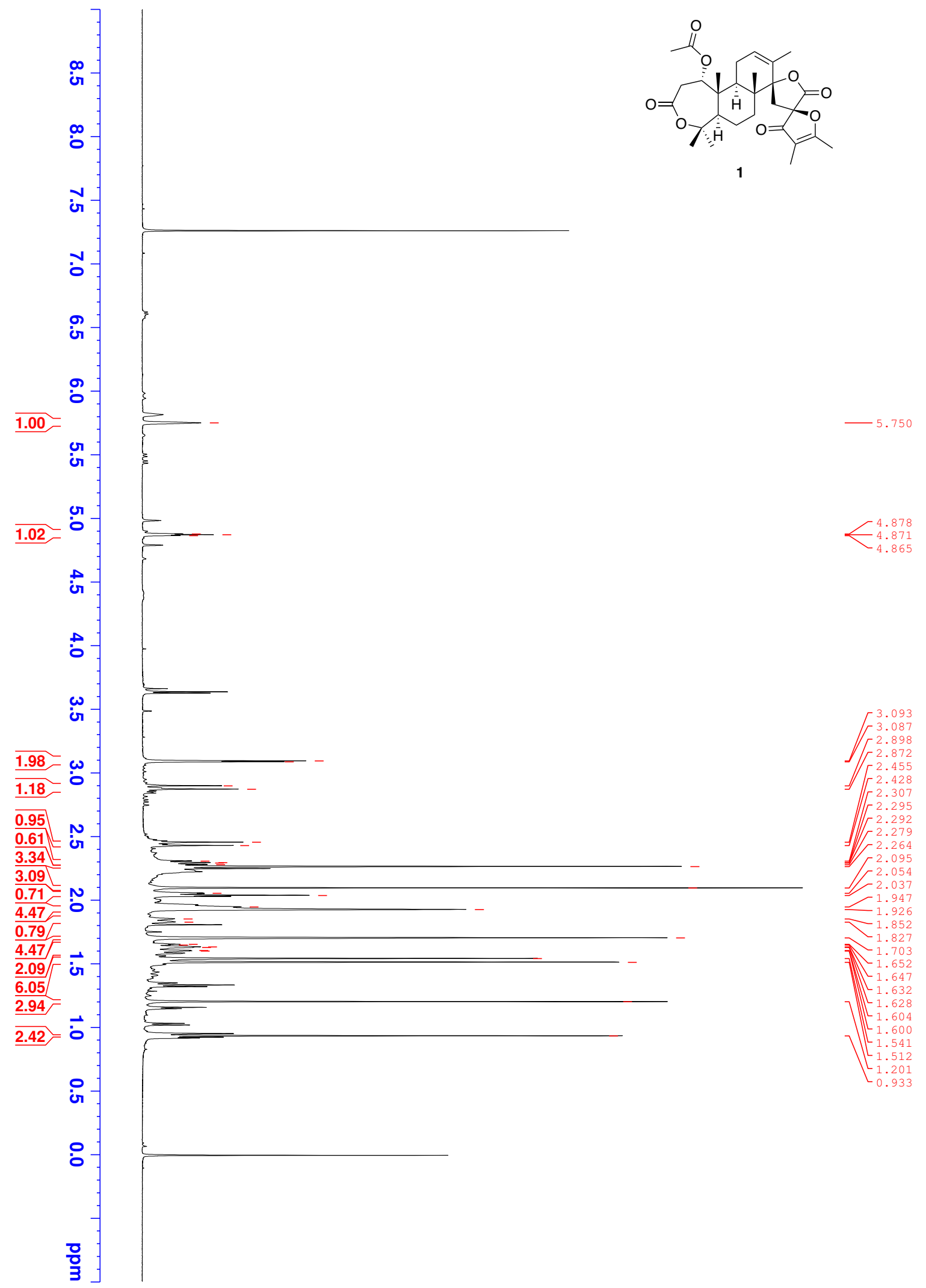

Figure S9. ${ }^{1} \mathrm{H}$ NMR spectrum of setosusin (1) in $\mathrm{CDCl}_{3}$ at $600 \mathrm{MHz}$. 


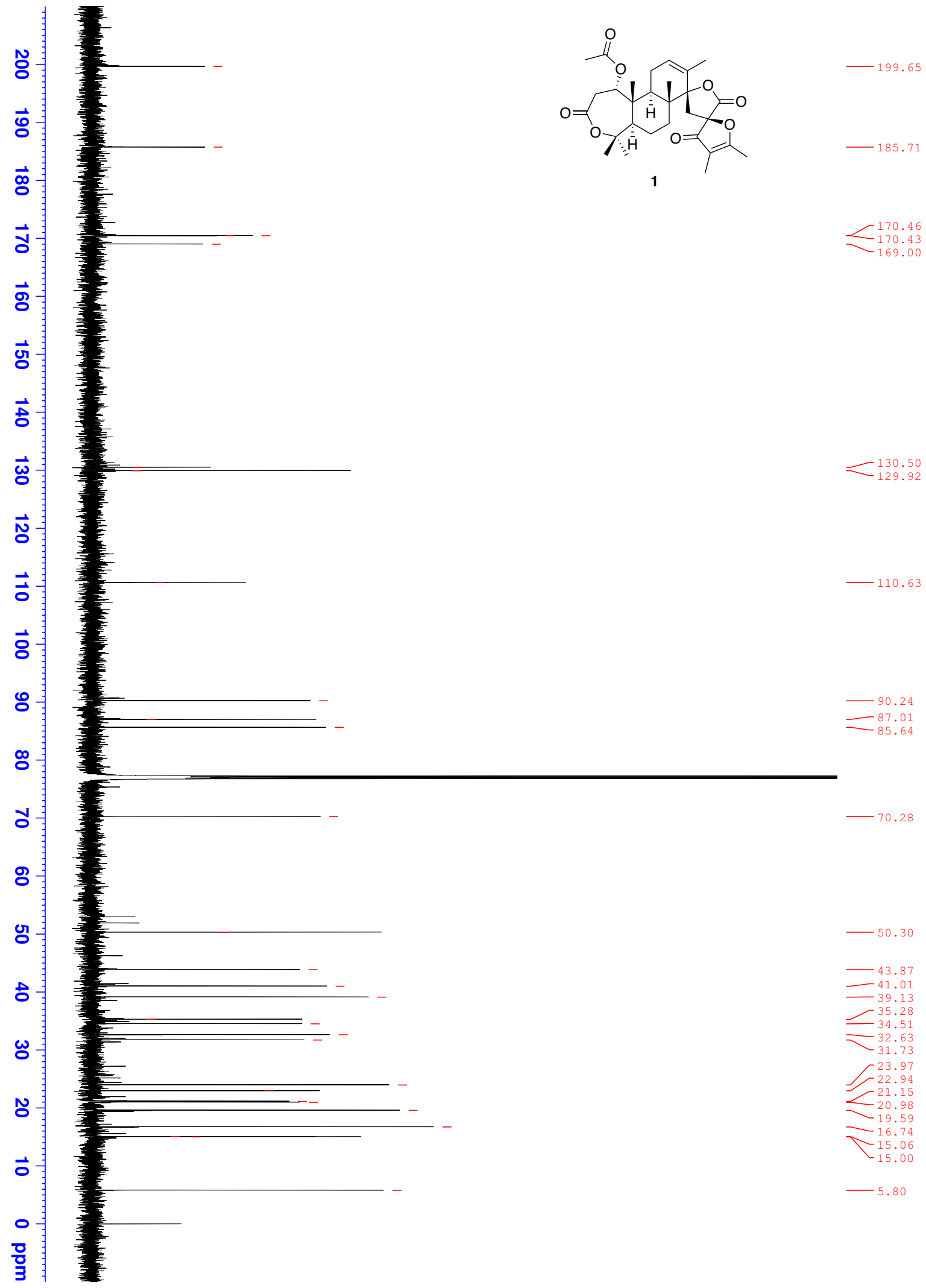

Figure S10. ${ }^{13} \mathrm{C}$ NMR spectrum of setosusin (1) in $\mathrm{CDCl}_{3}$ at $150 \mathrm{MHz}$. 


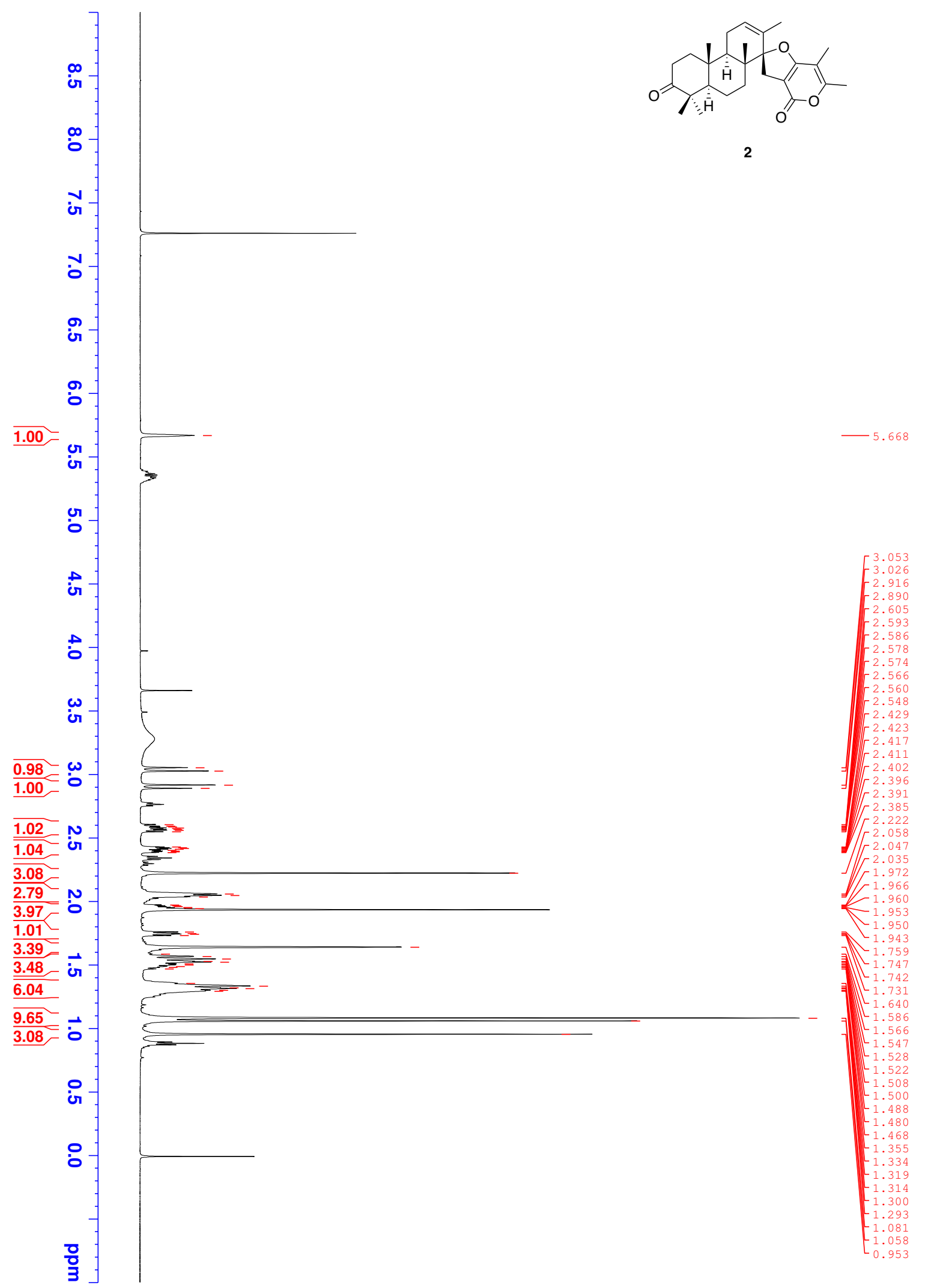

Figure S11. ${ }^{1} \mathrm{H}$ NMR spectrum of brevione $\mathrm{B}(2)$ in $\mathrm{CDCl}_{3}$ at $600 \mathrm{MHz}$. 


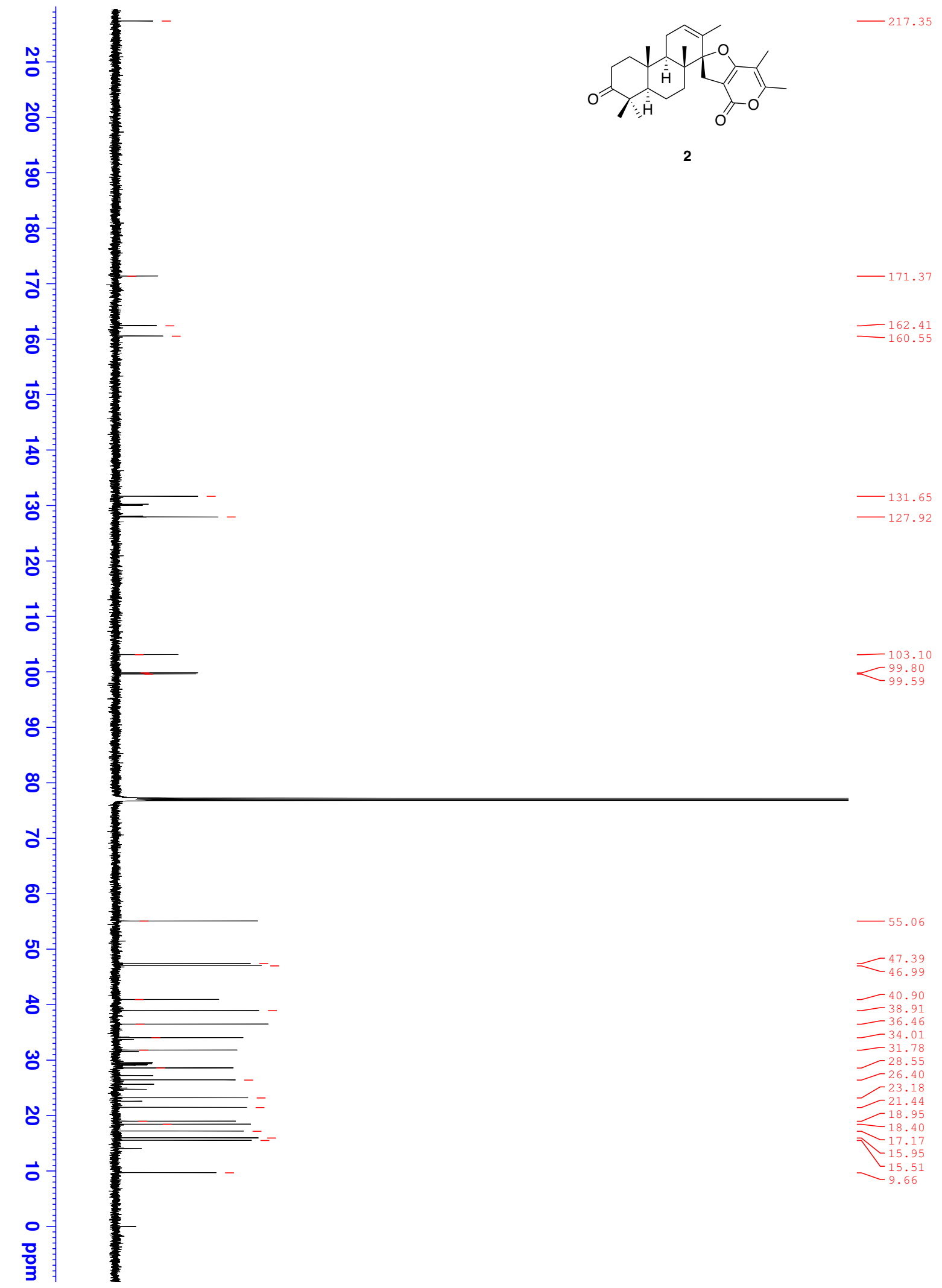

Figure $\mathrm{S} 12 .{ }^{13} \mathrm{C}$ NMR spectrum of brevione $\mathrm{B}(2)$ in $\mathrm{CDCl}_{3}$ at $150 \mathrm{MHz}$. 


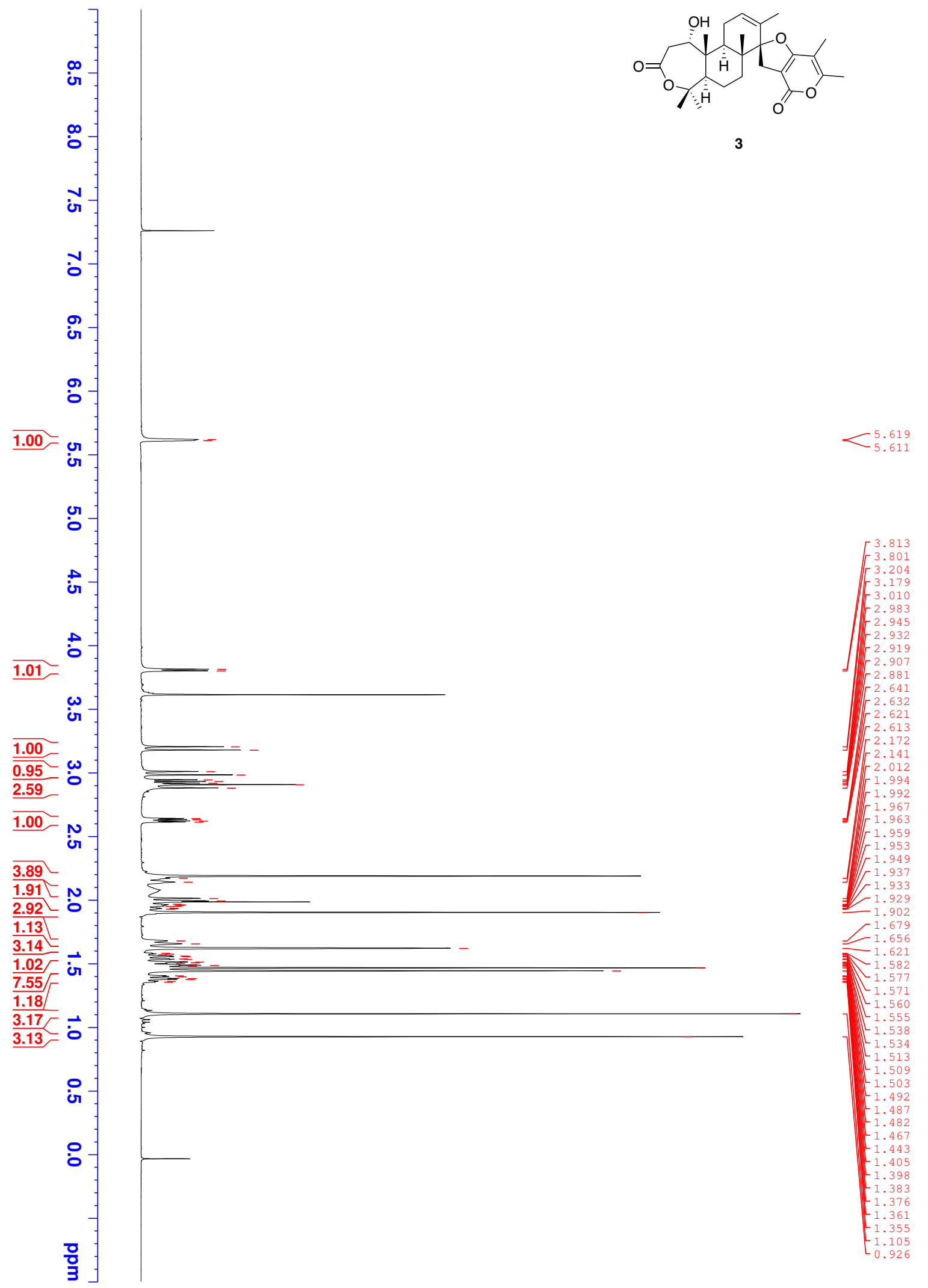

Figure $\mathrm{S} 13 .{ }^{1} \mathrm{H}$ NMR spectrum of brevione $\mathrm{L}(3)$ in $\mathrm{CDCl}_{3}$ at $600 \mathrm{MHz}$. 


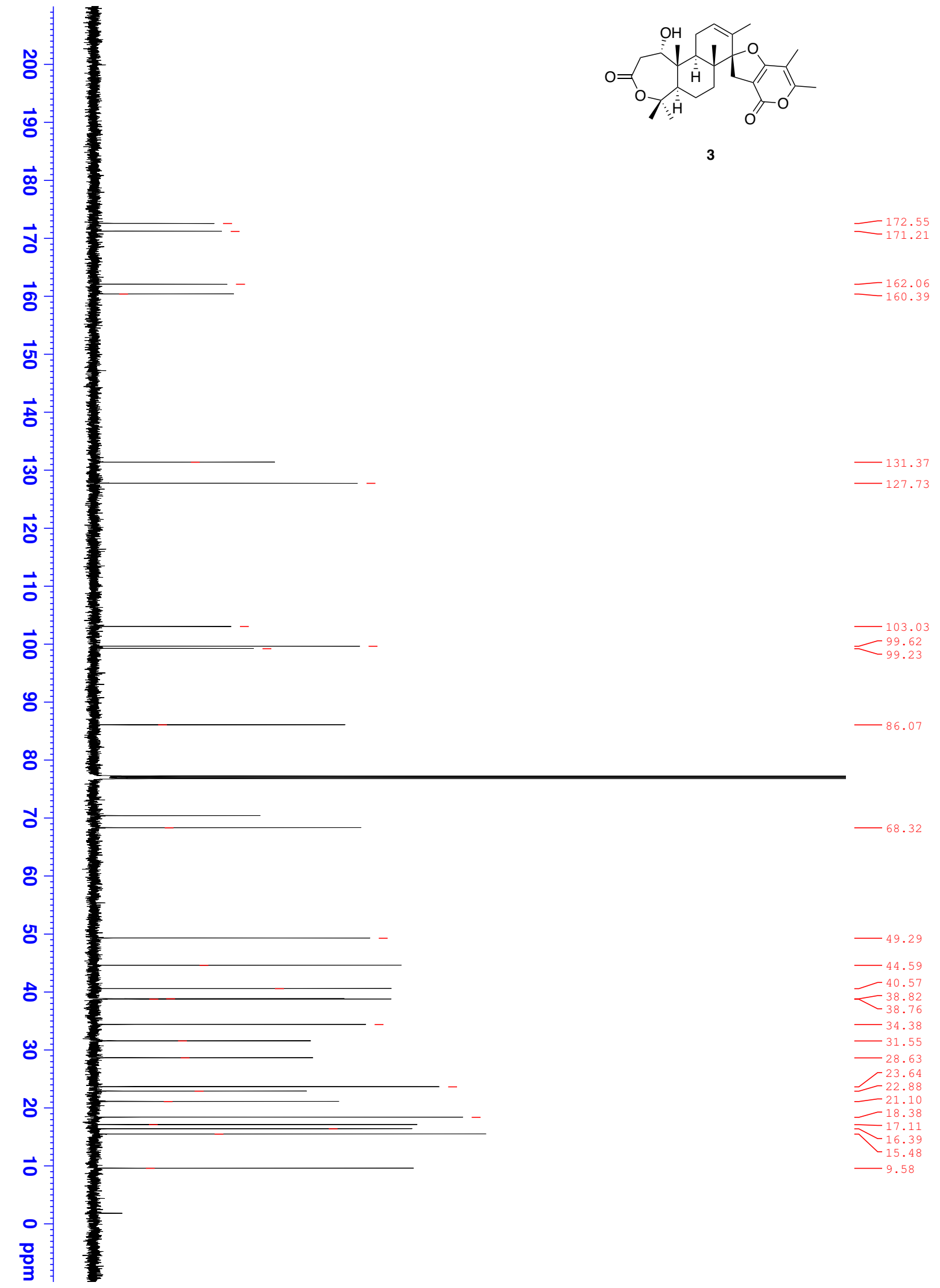

Figure S14. ${ }^{13} \mathrm{C}$ NMR spectrum of brevione $\mathrm{L}(3)$ in $\mathrm{CDCl}_{3}$ at $150 \mathrm{MHz}$. 


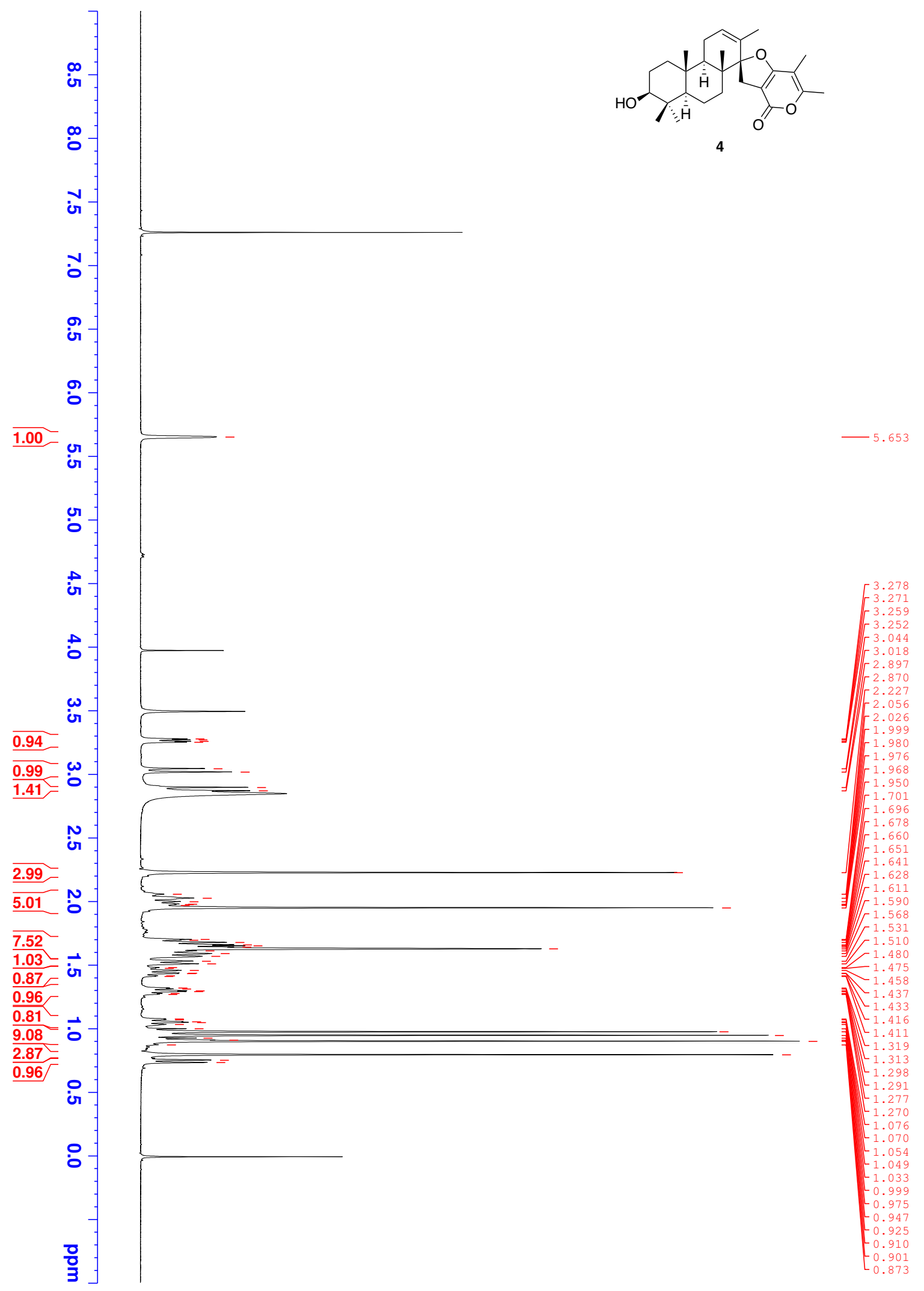

Figure S15. ${ }^{1} \mathrm{H}$ NMR spectrum of brevione $\mathrm{N}(4)$ in $\mathrm{CDCl}_{3}$ at $600 \mathrm{MHz}$. 


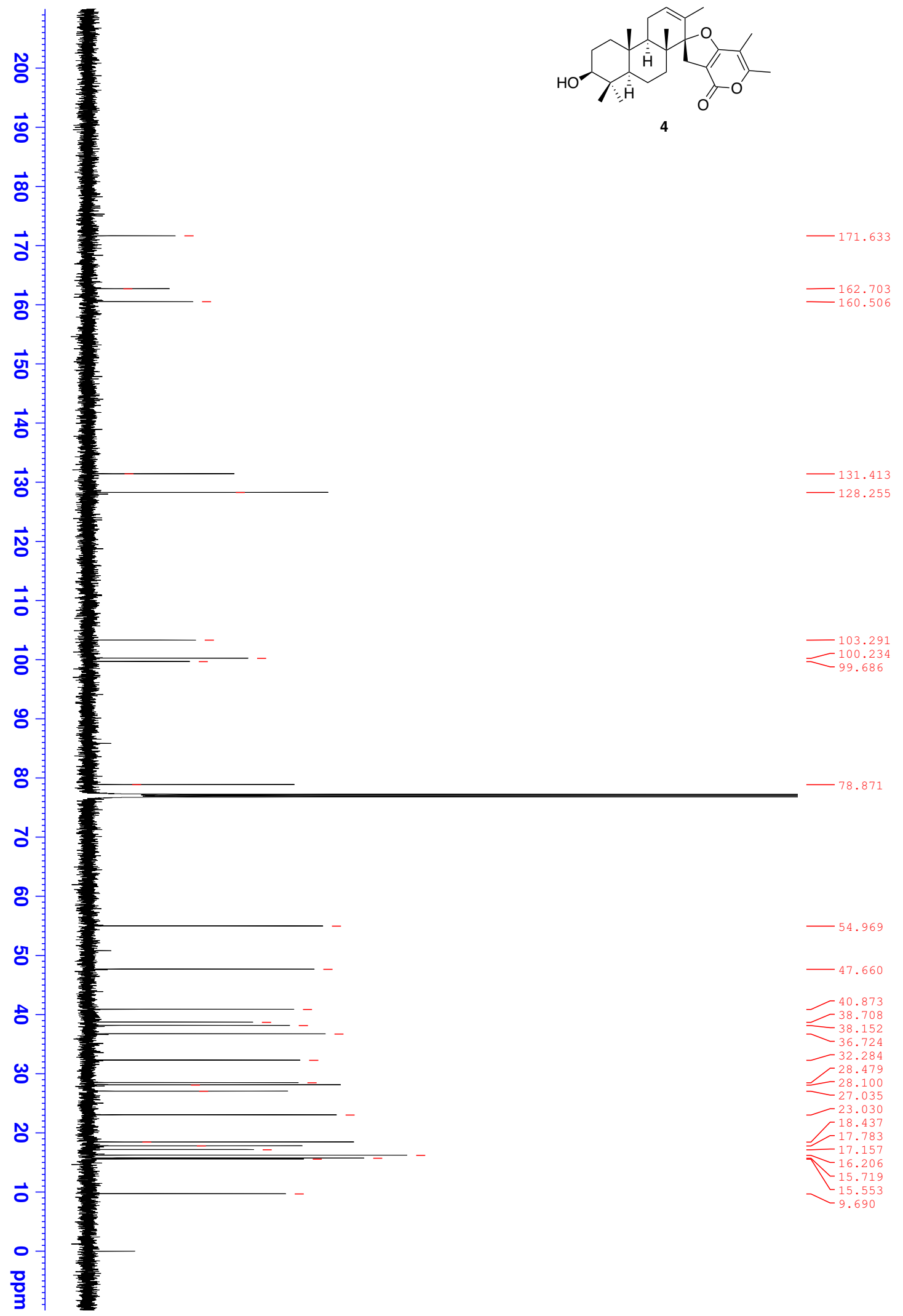

Figure S16. ${ }^{13} \mathrm{C}$ NMR spectrum of brevione $\mathrm{N}(4)$ in $\mathrm{CDCl}_{3}$ at $150 \mathrm{MHz}$. 


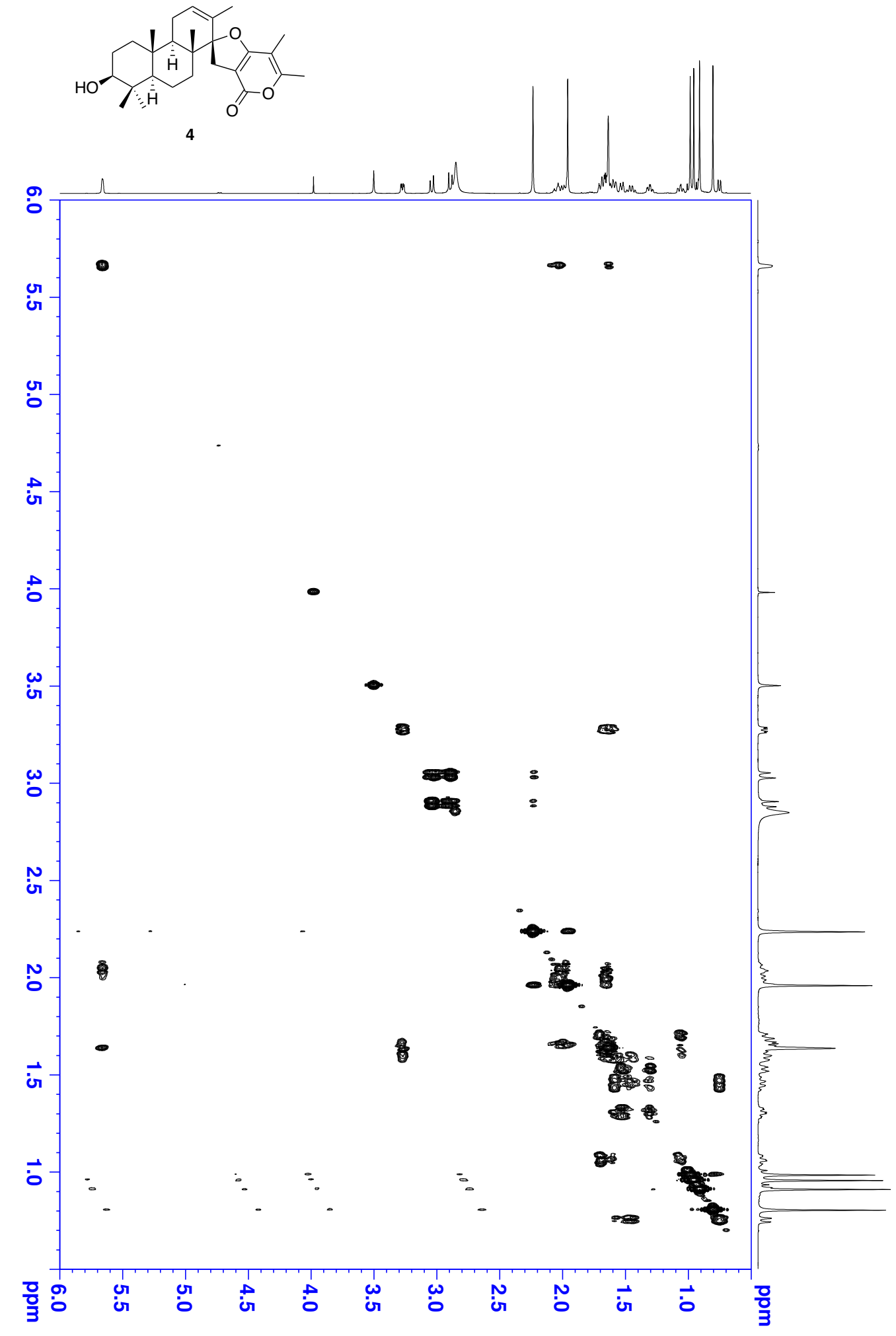

Figure S17. ${ }^{1} \mathrm{H}-{ }^{1} \mathrm{H}$ COSY spectrum of brevione $\mathrm{N}(4)$ in $\mathrm{CDCl}_{3}$. 


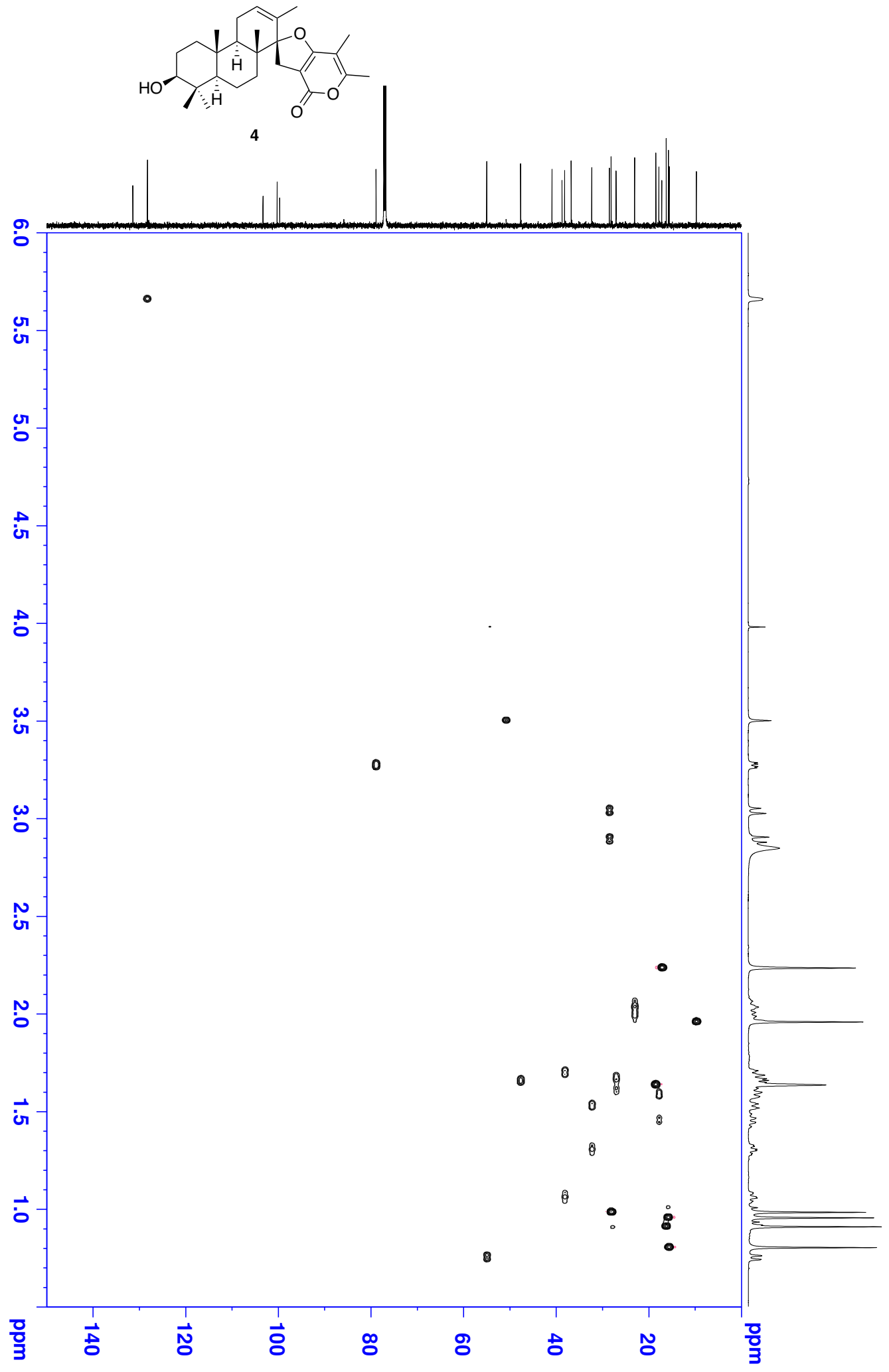

Figure S18. HSQC spectrum of brevione $\mathrm{N}(4)$ in $\mathrm{CDCl}_{3}$. 


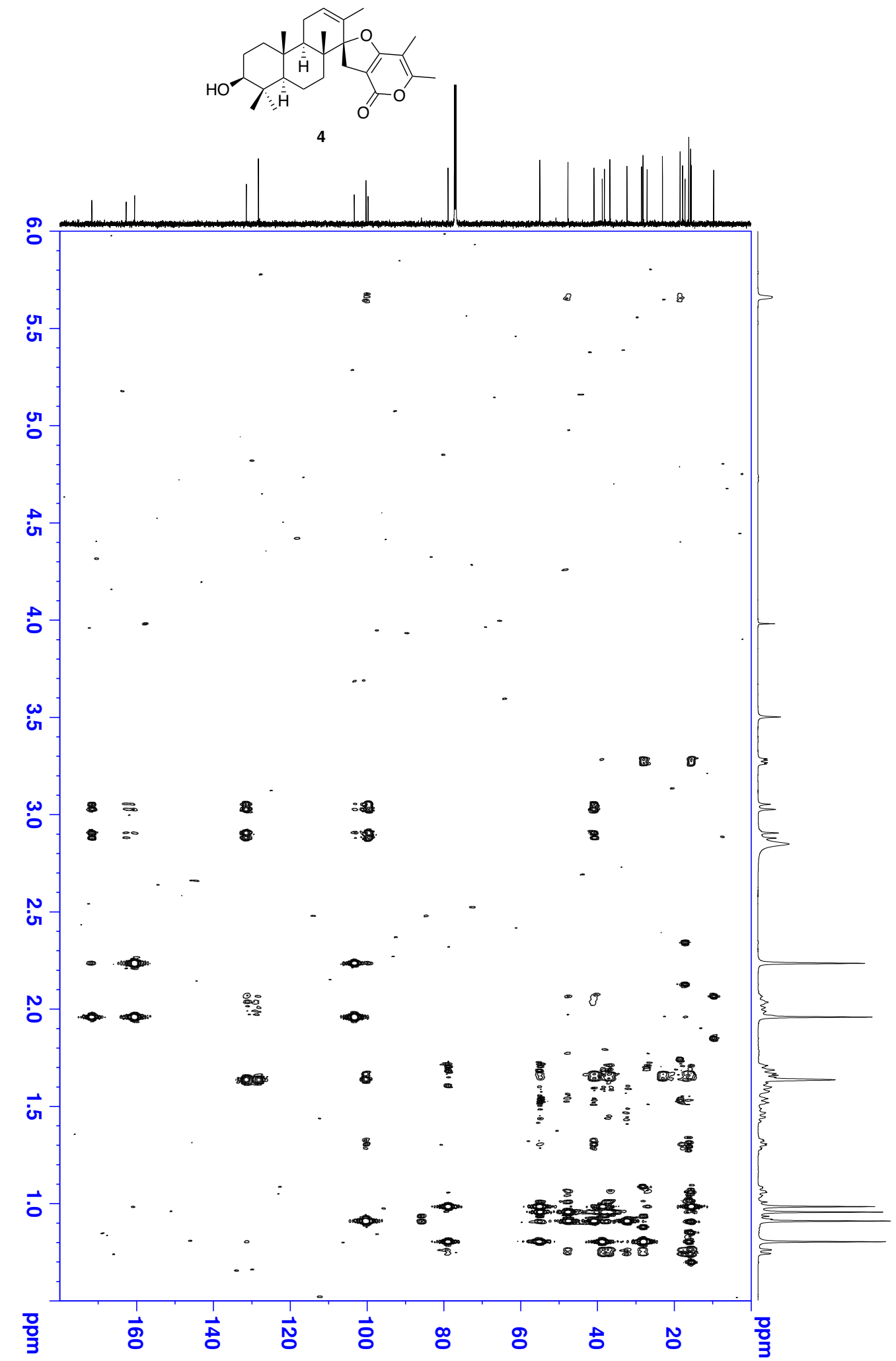

Figure S19. HMBC of brevione $\mathrm{N}(4)$ in $\mathrm{CDCl}_{3}$. 


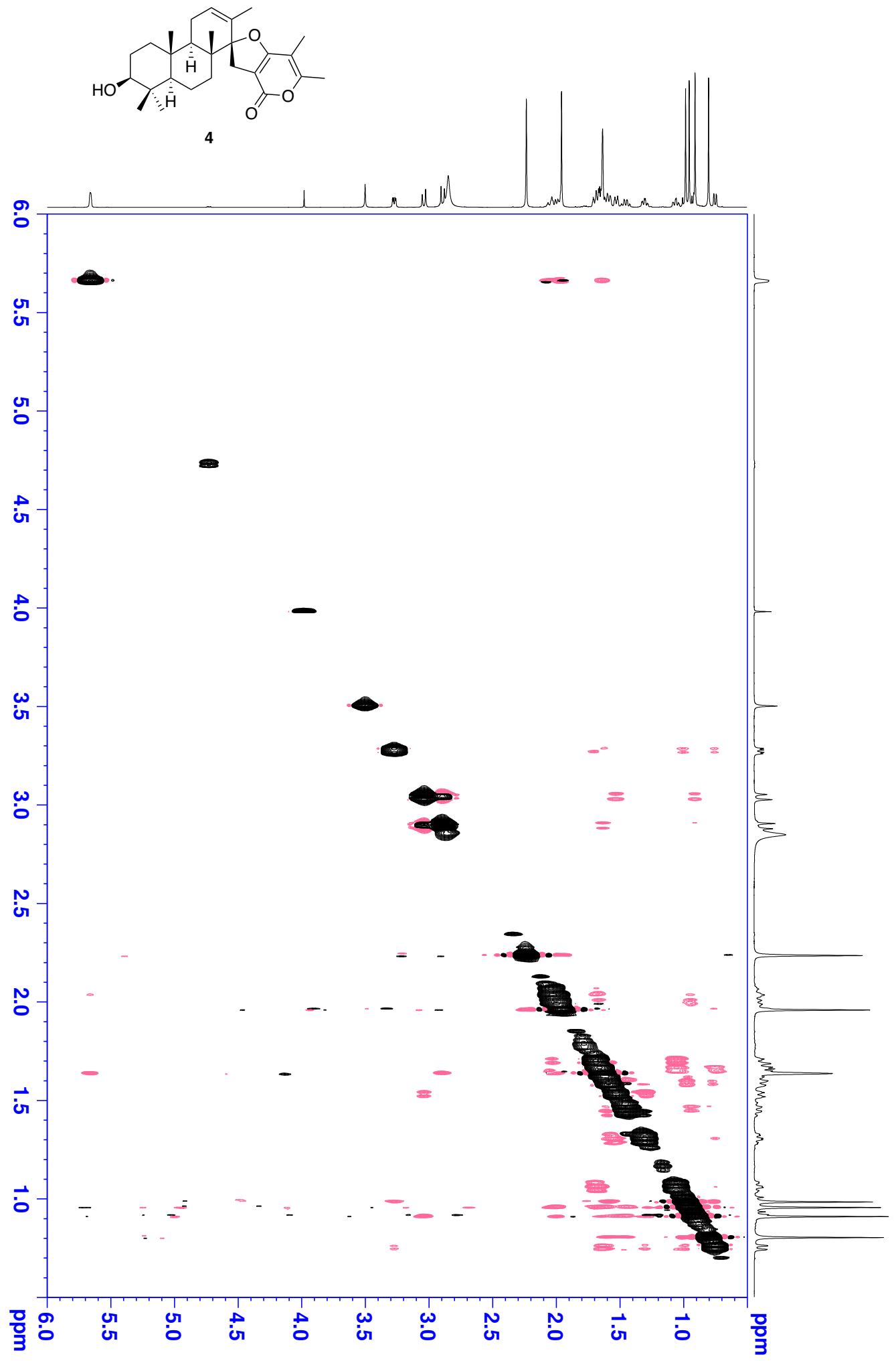

Figure S20. NOESY spectrum of brevione $\mathrm{N}(4)$ in $\mathrm{CDCl}_{3}$. 


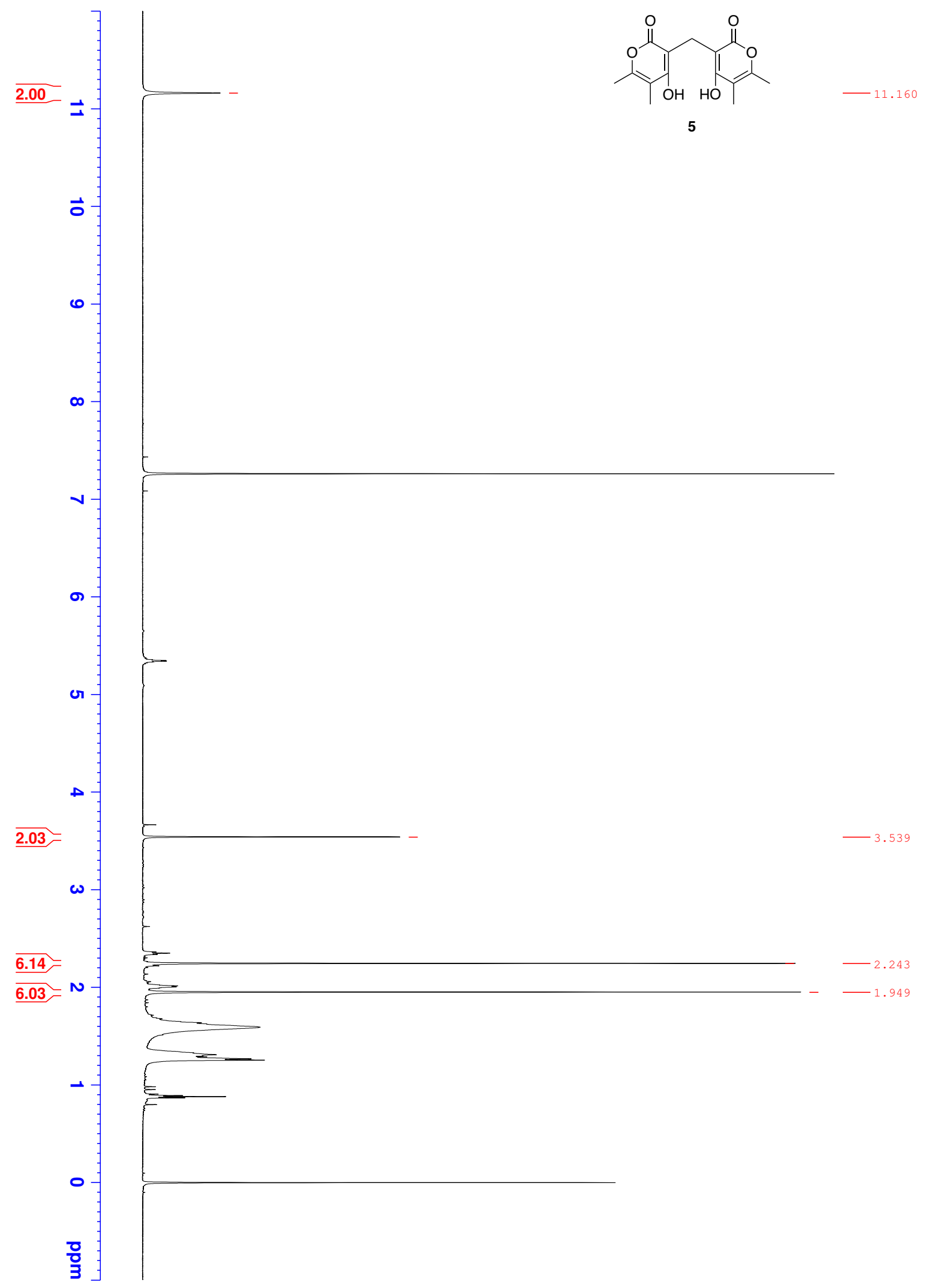

Figure S21. ${ }^{1} \mathrm{H}$ NMR spectrum of colletopyrone (5) in $\mathrm{CDCl}_{3}$ at $600 \mathrm{MHz}$. 


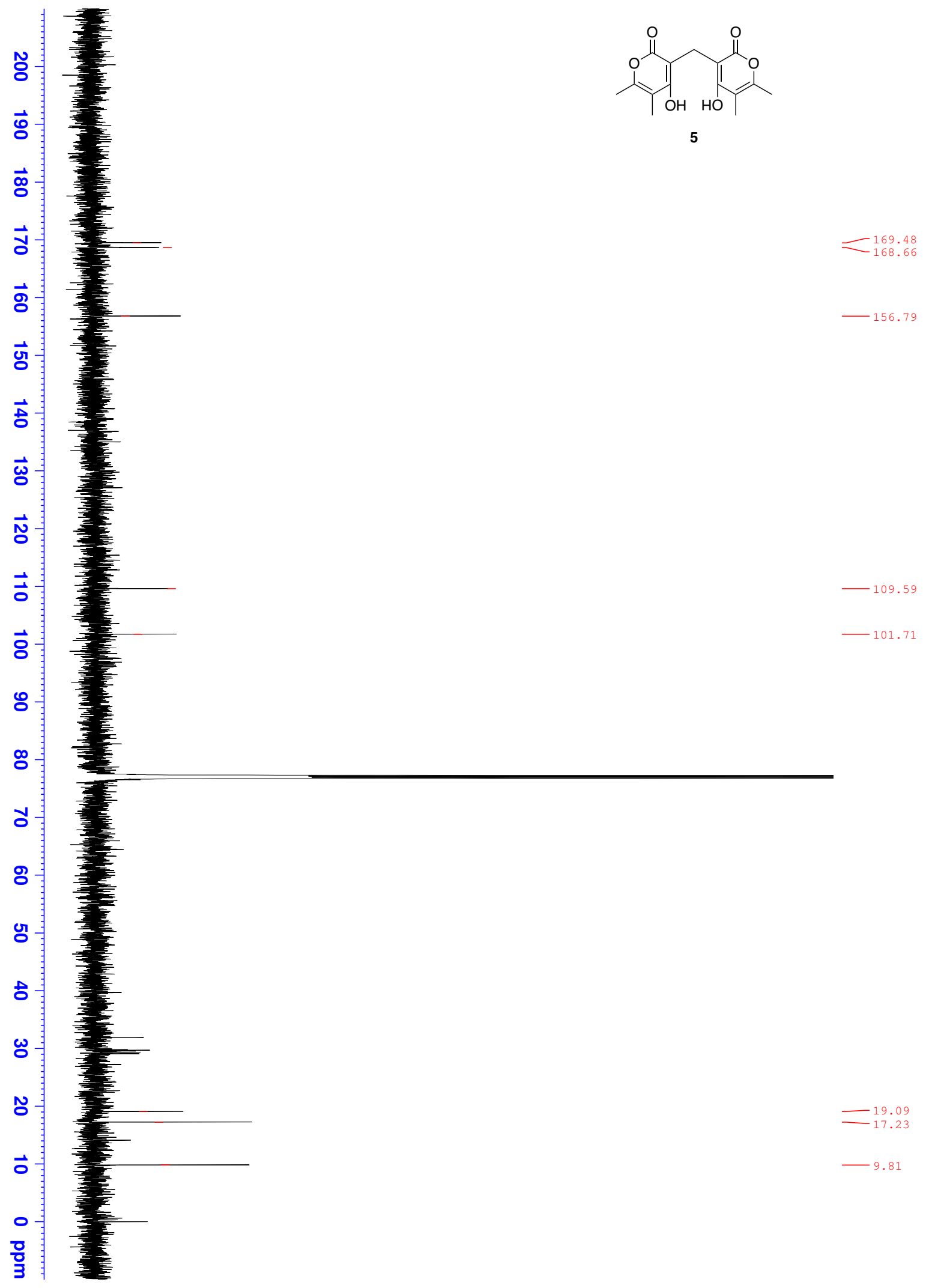

Figure S22. ${ }^{13} \mathrm{C}$ NMR spectrum of colletopyrone (5) in $\mathrm{CDCl}_{3}$ at $150 \mathrm{MHz}$. 


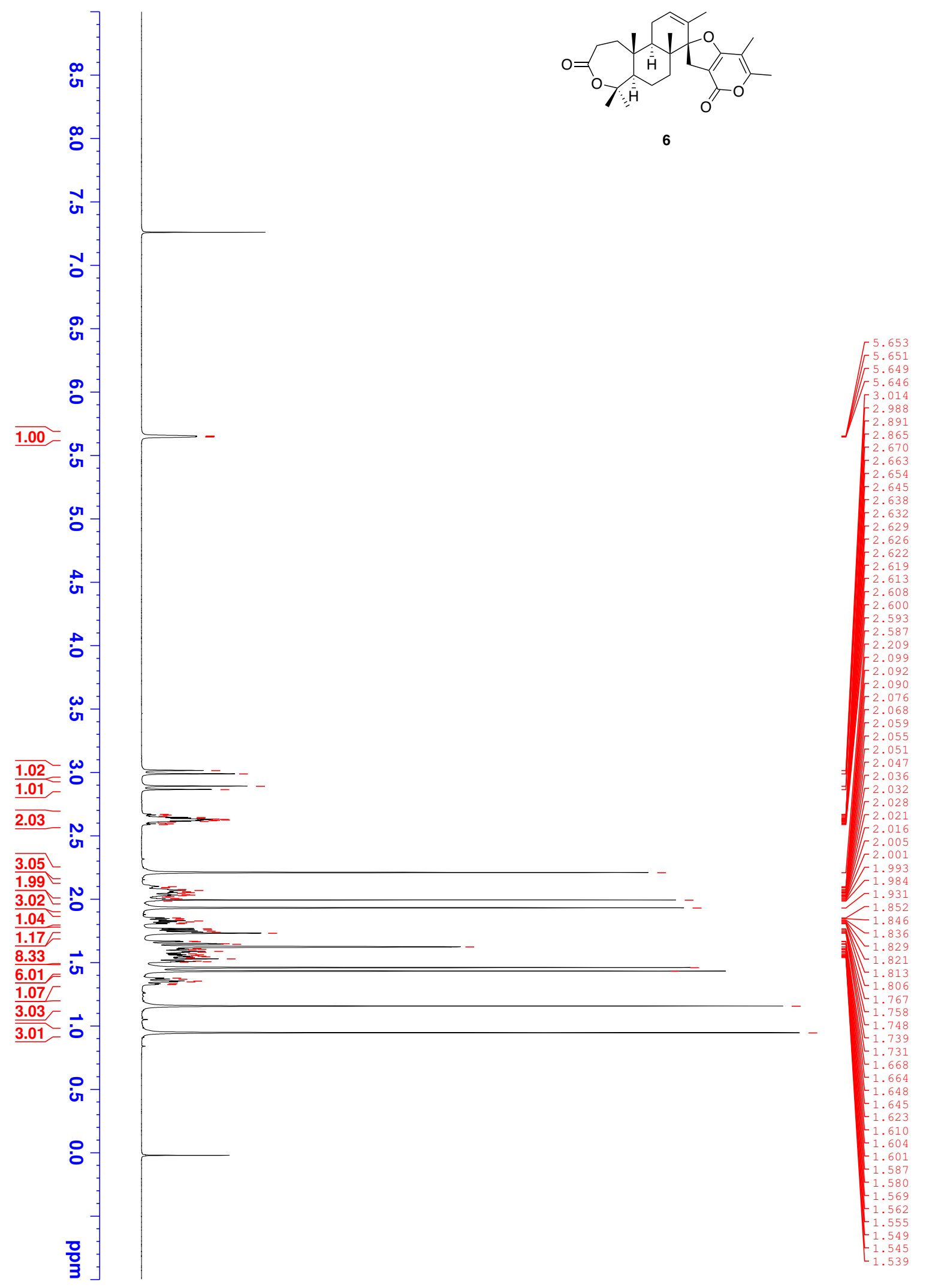

Figure S23. ${ }^{1} \mathrm{H}$ NMR spectrum of brevione $\mathrm{R}(6)$ in $\mathrm{CDCl}_{3}$ at $600 \mathrm{MHz}$. 


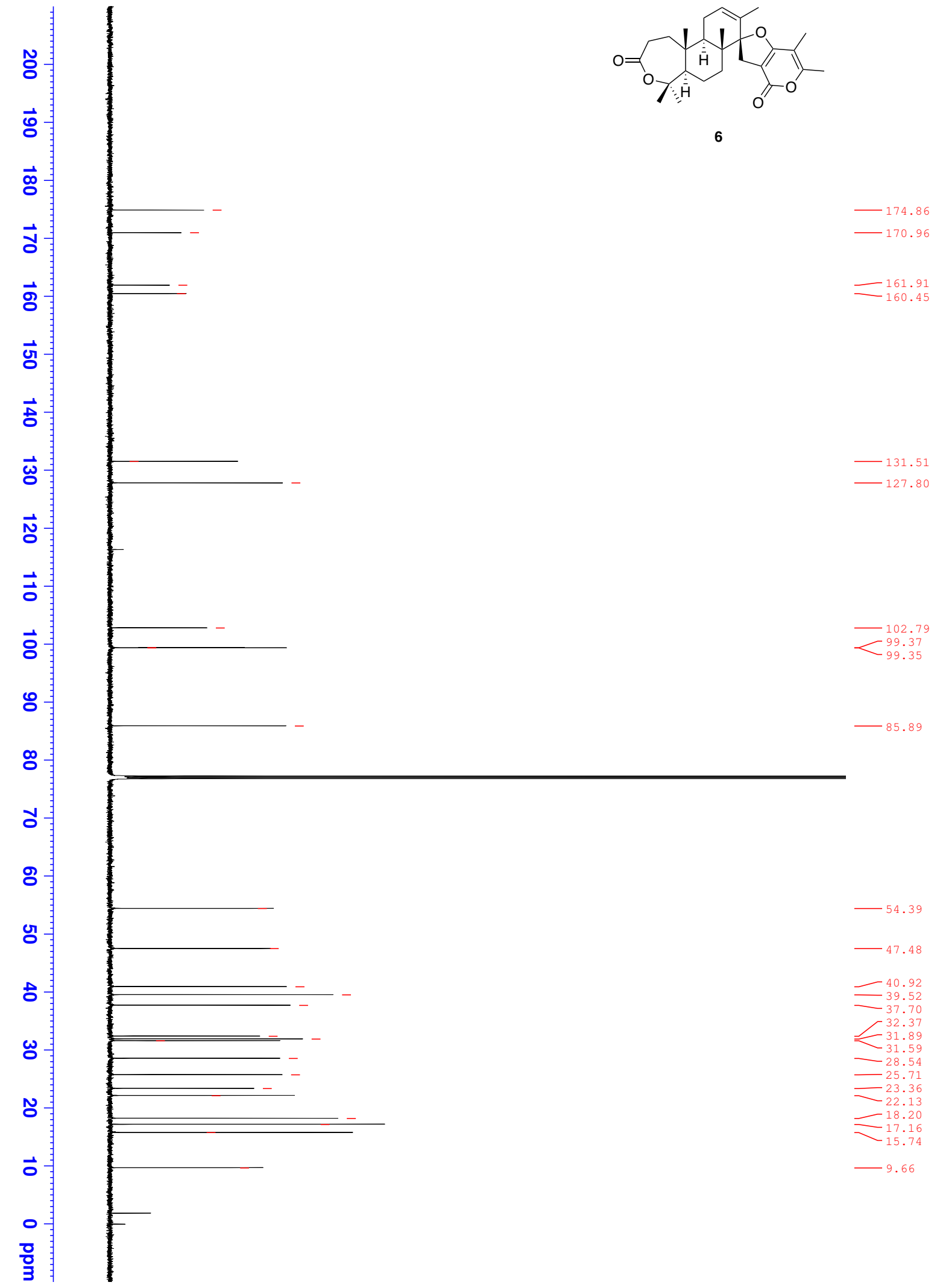

Figure S24. ${ }^{13} \mathrm{C}$ NMR spectrum of brevione $\mathrm{R}(6)$ in $\mathrm{CDCl}_{3}$ at $150 \mathrm{MHz}$. 


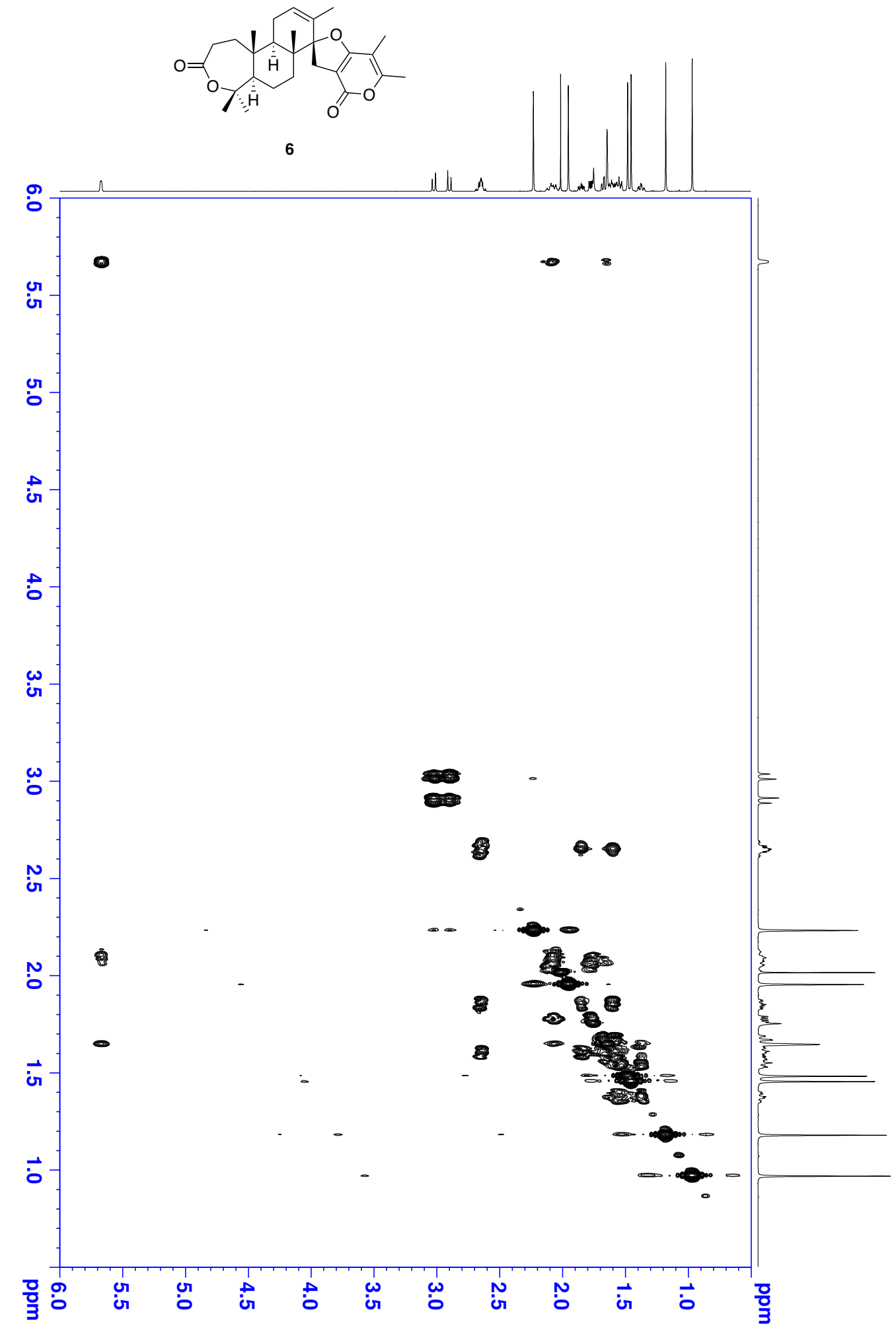

Figure S25. ${ }^{1} \mathrm{H}-{ }^{1} \mathrm{H}$ COSY spectrum of brevione $\mathrm{R}(6)$ in $\mathrm{CDCl}_{3}$. 


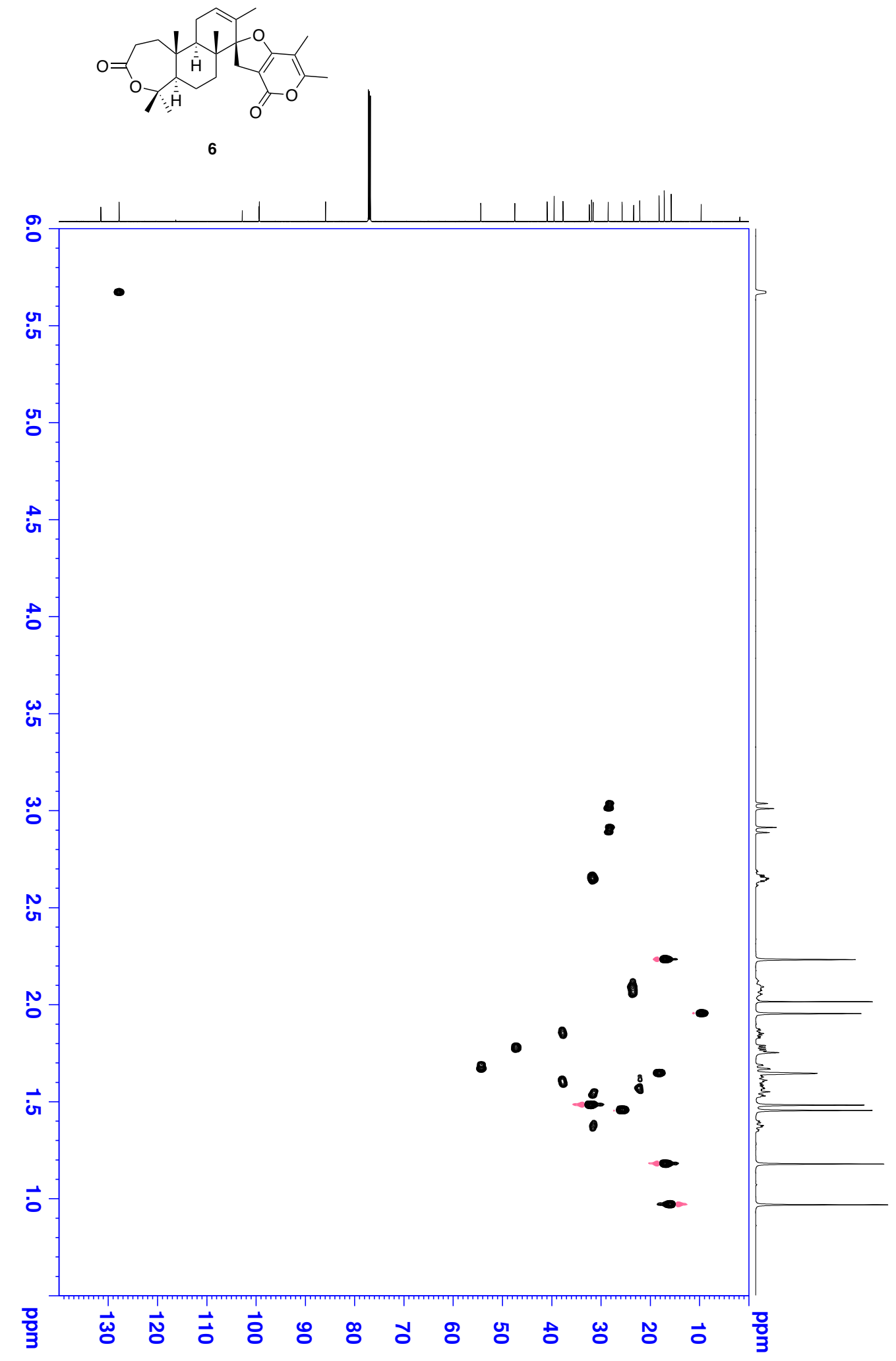

Figure S26. HSQC spectrum of brevione R (6) in $\mathrm{CDCl}_{3}$. 


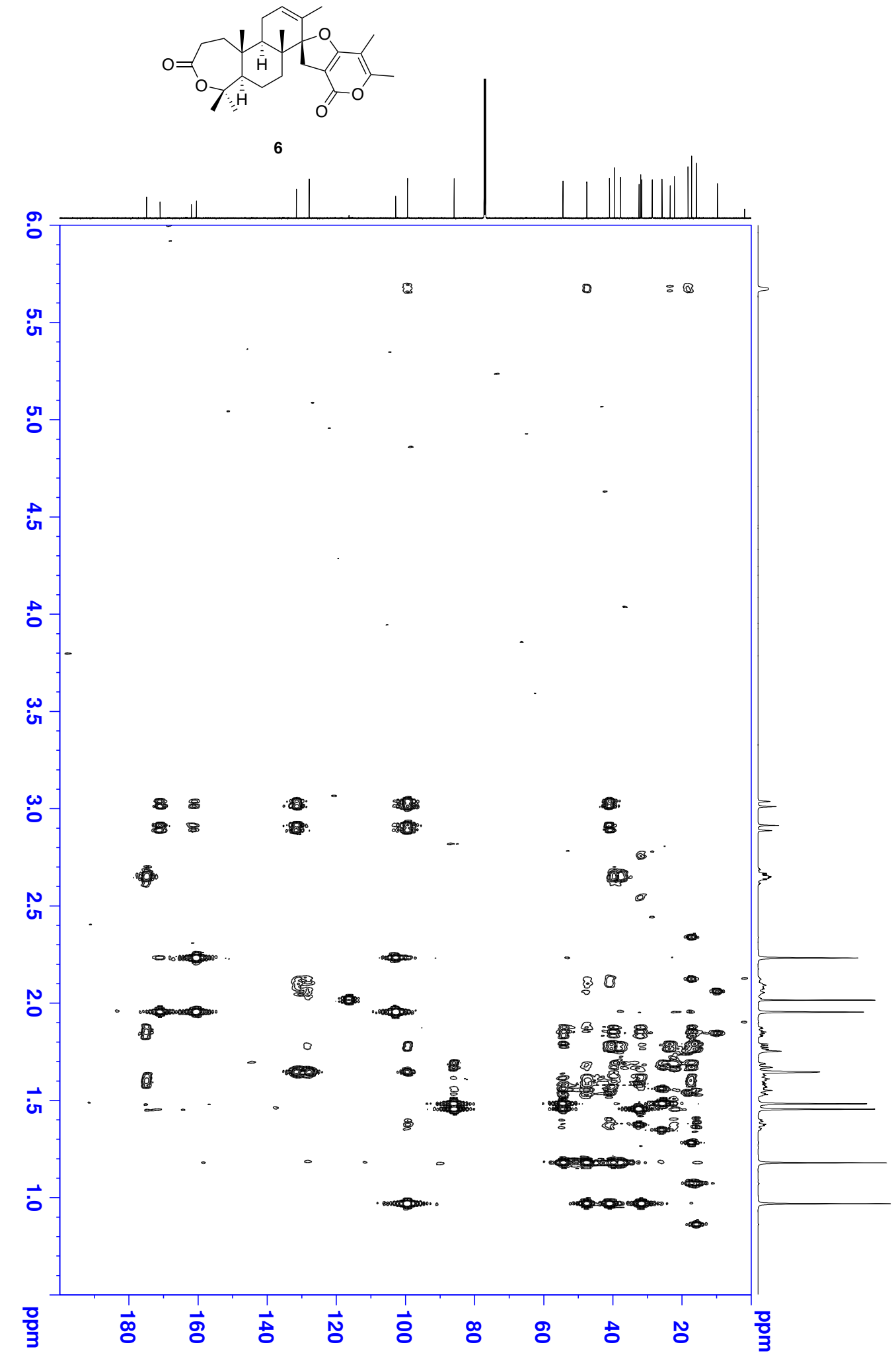

Figure S27. $\mathrm{HMBC}$ of brevione $\mathrm{R}(\mathbf{6})$ in $\mathrm{CDCl}_{3}$. 


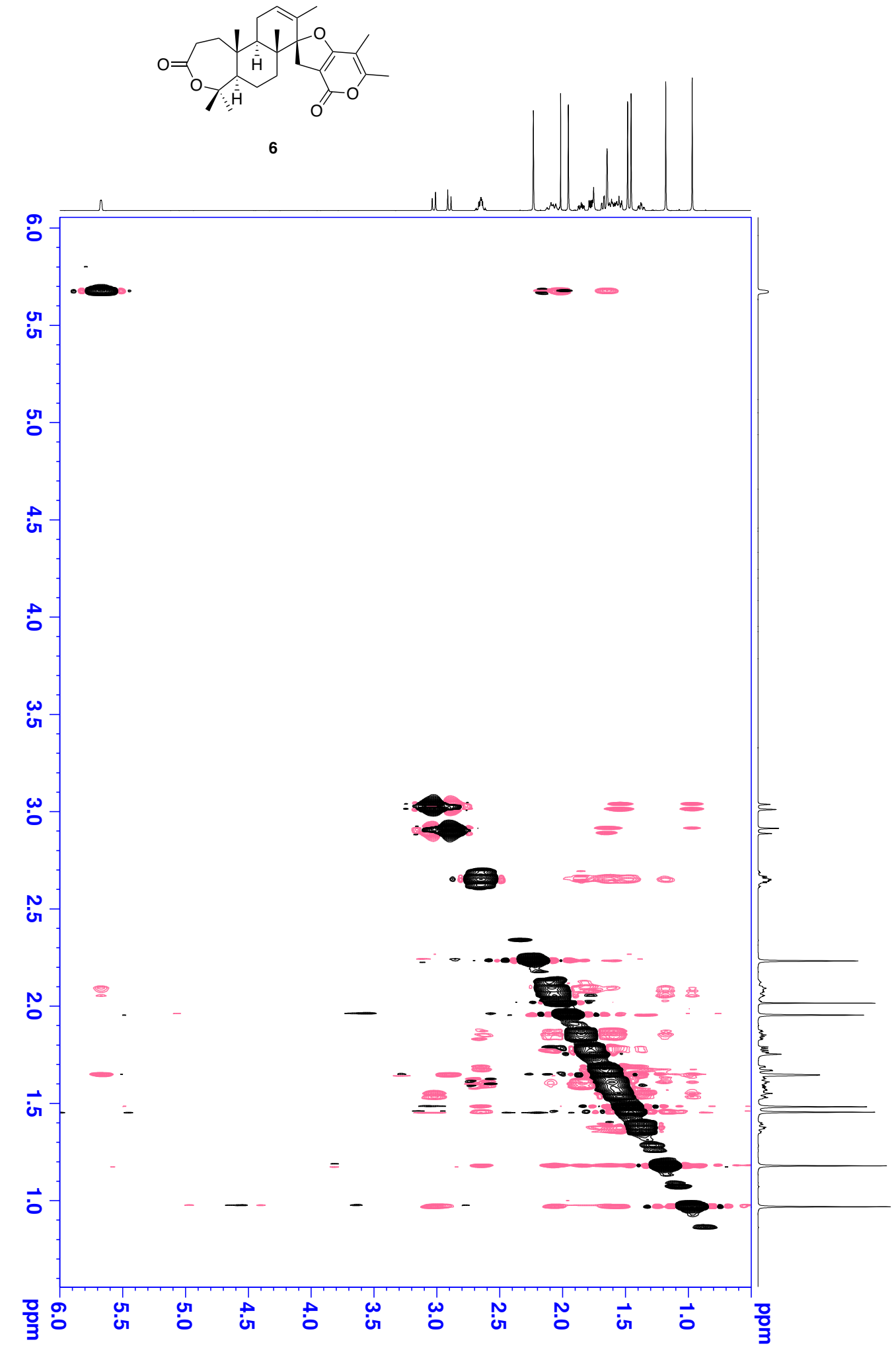

Figure S28. NOESY spectrum of brevione $\mathrm{R}(\mathbf{6})$ in $\mathrm{CDCl}_{3}$. 


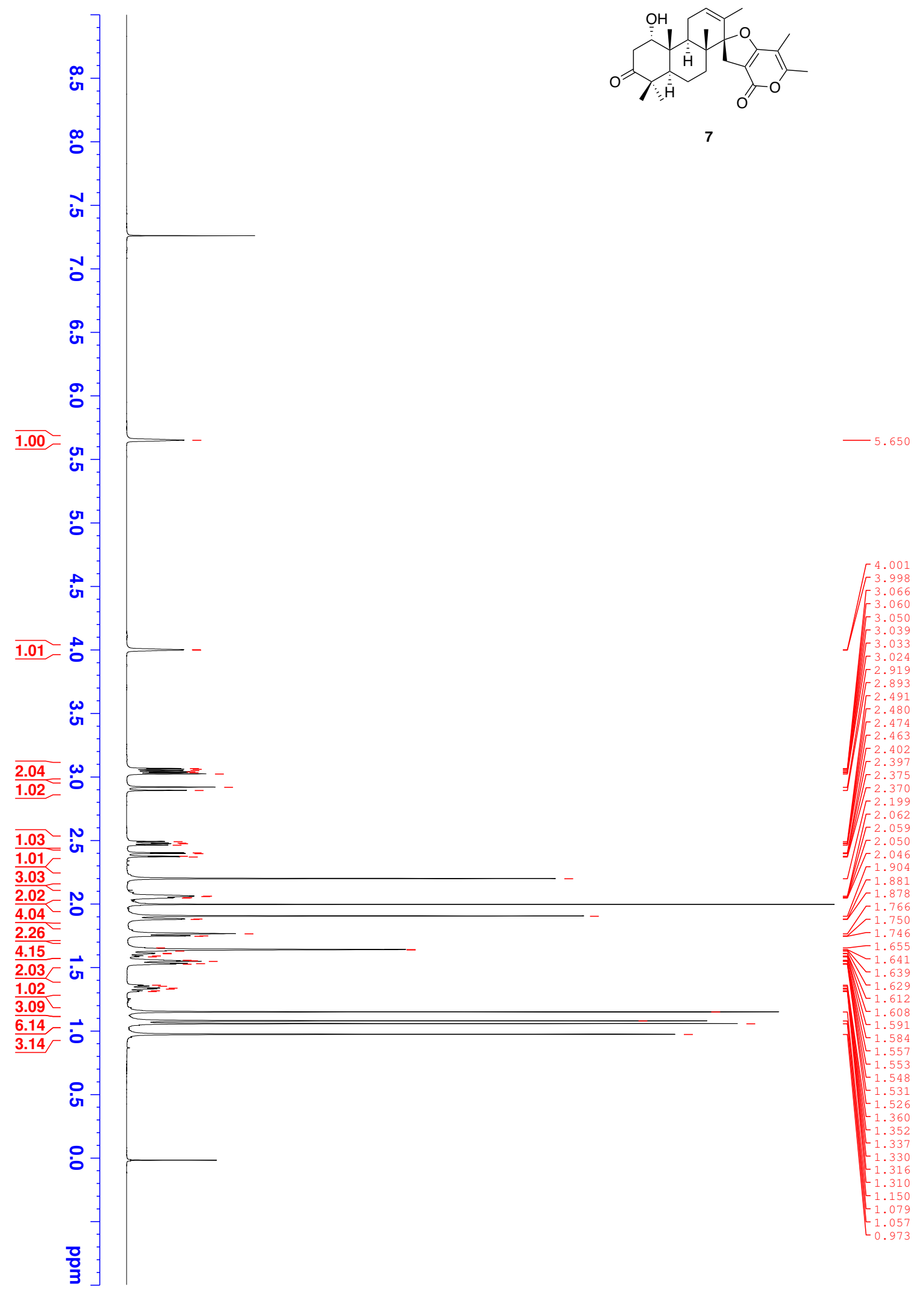

Figure S29. ${ }^{1} \mathrm{H}$ NMR spectrum of brevione $\mathrm{S}(7)$ in $\mathrm{CDCl}_{3}$ at $600 \mathrm{MHz}$. 


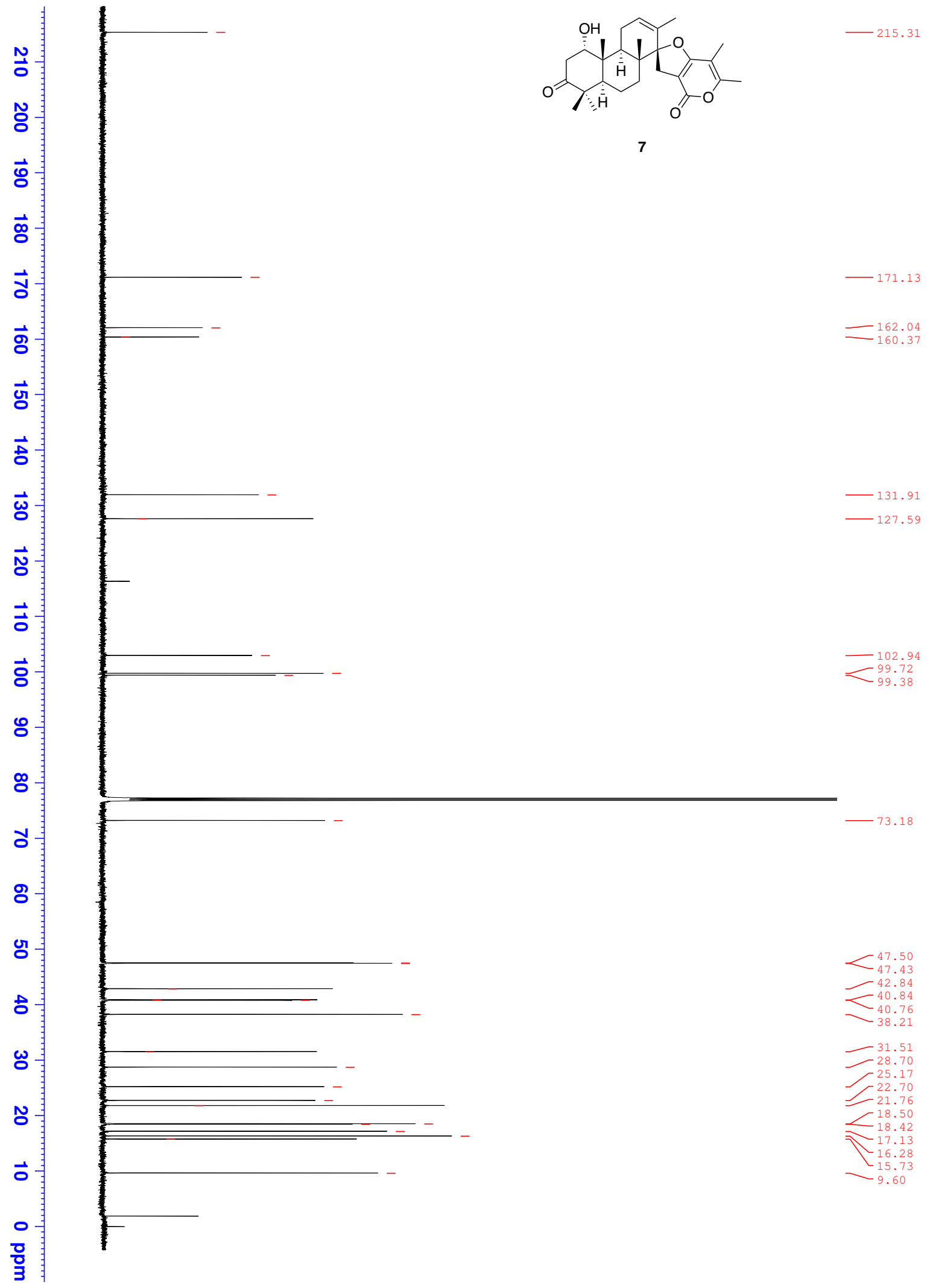

Figure $\mathrm{S} 30 .{ }^{13} \mathrm{C}$ NMR spectrum of brevione $\mathrm{S}(7)$ in $\mathrm{CDCl}_{3}$ at $150 \mathrm{MHz}$. 


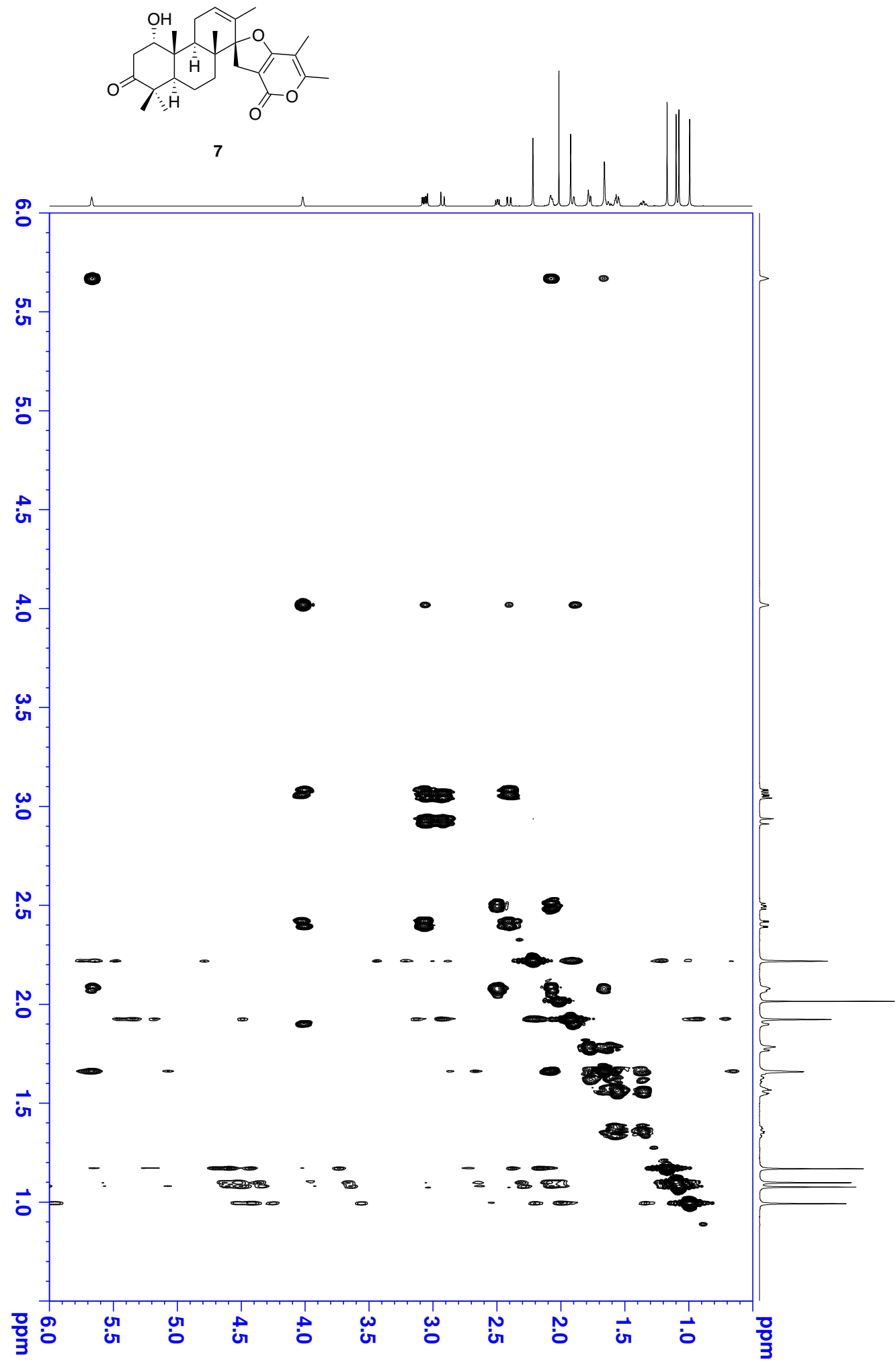

Figure $\mathrm{S} 31 .{ }^{1} \mathrm{H}-{ }^{1} \mathrm{H}$ COSY spectrum of brevione $\mathrm{S}(7)$ in $\mathrm{CDCl}_{3}$. 


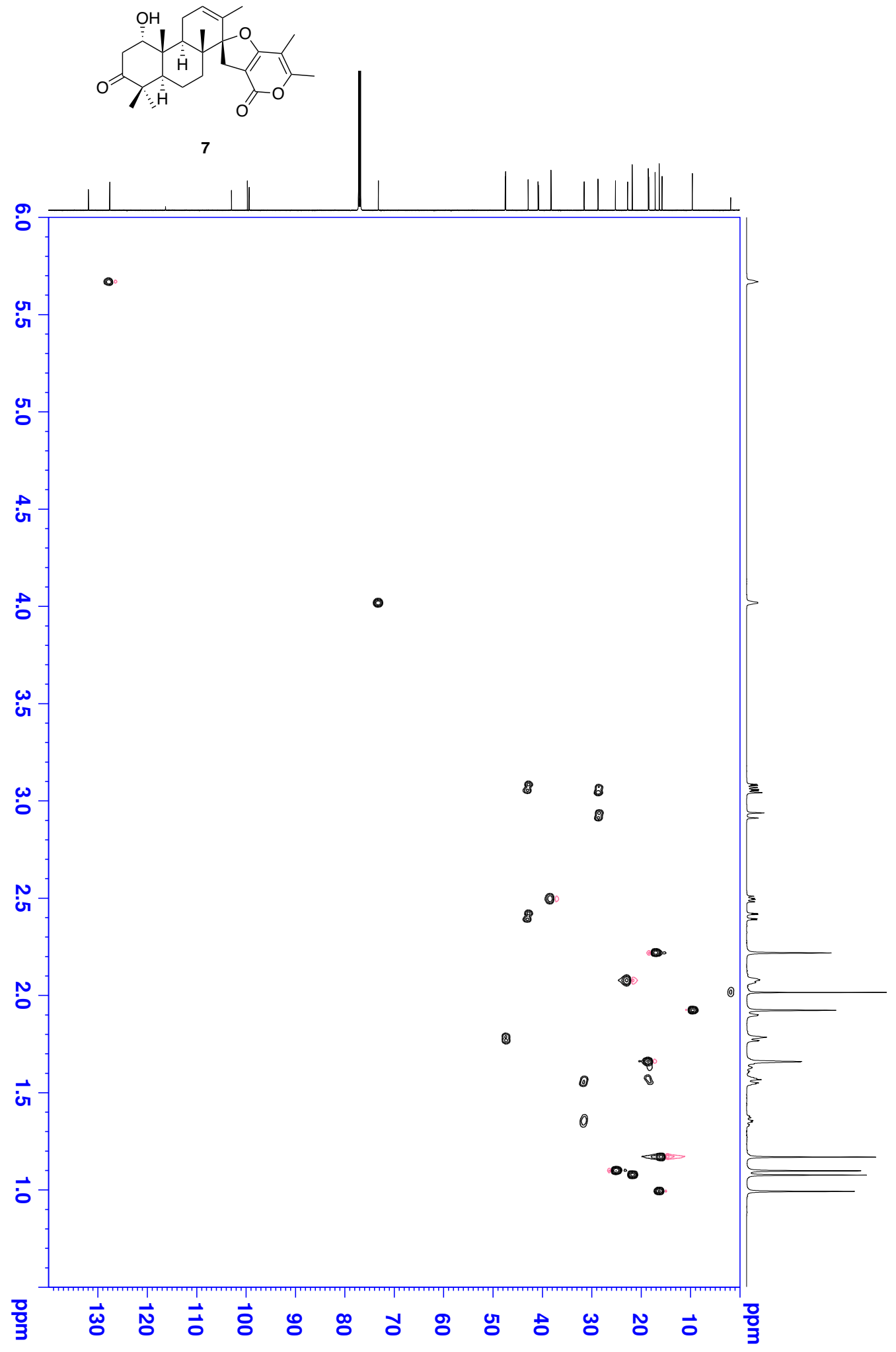

Figure S32. HSQC spectrum of brevione $\mathrm{S}(7)$ in $\mathrm{CDCl}_{3}$. 


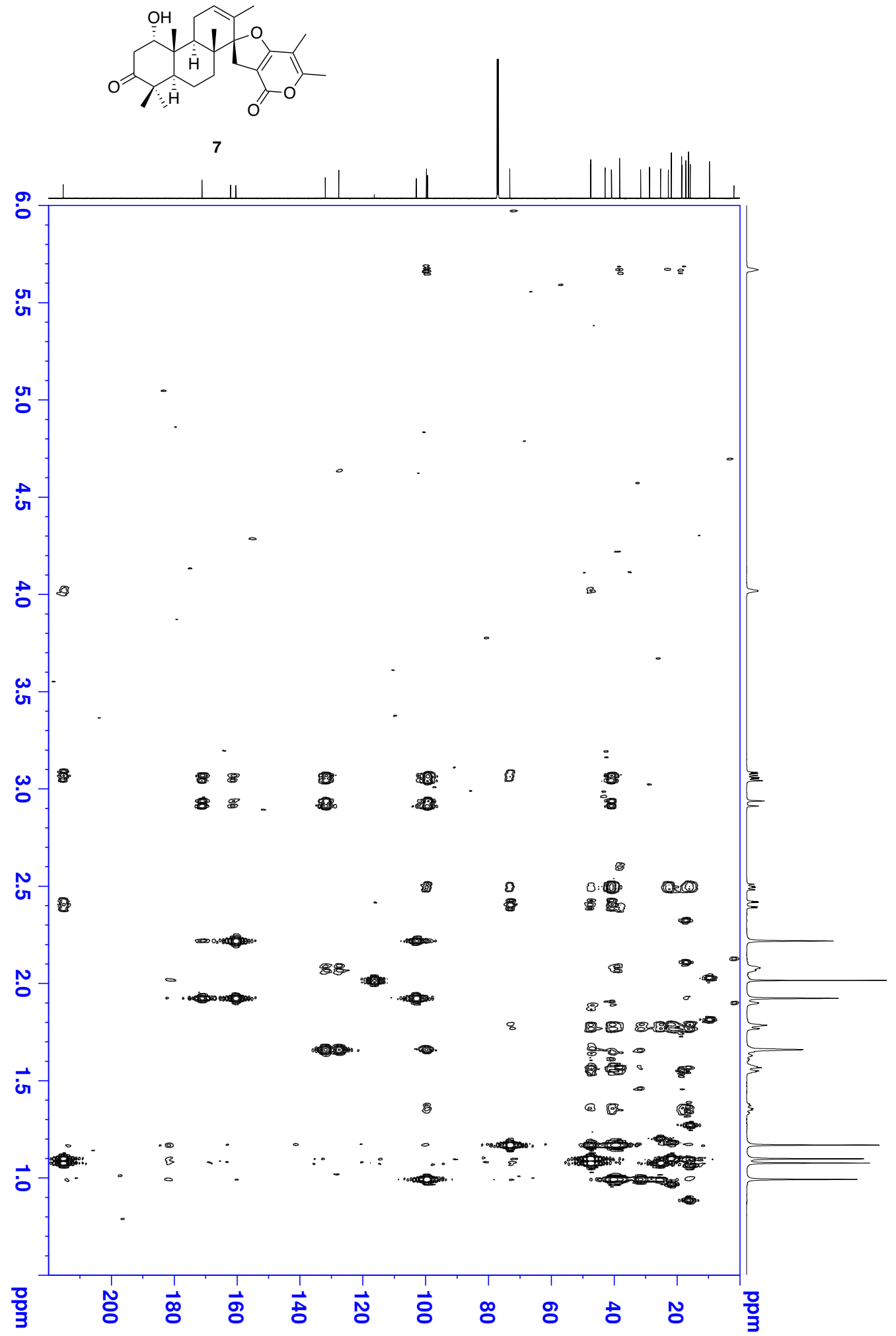

Figure $\mathrm{S} 33$. $\mathrm{HMBC}$ of brevione $\mathrm{S}(7)$ in $\mathrm{CDCl}_{3}$. 


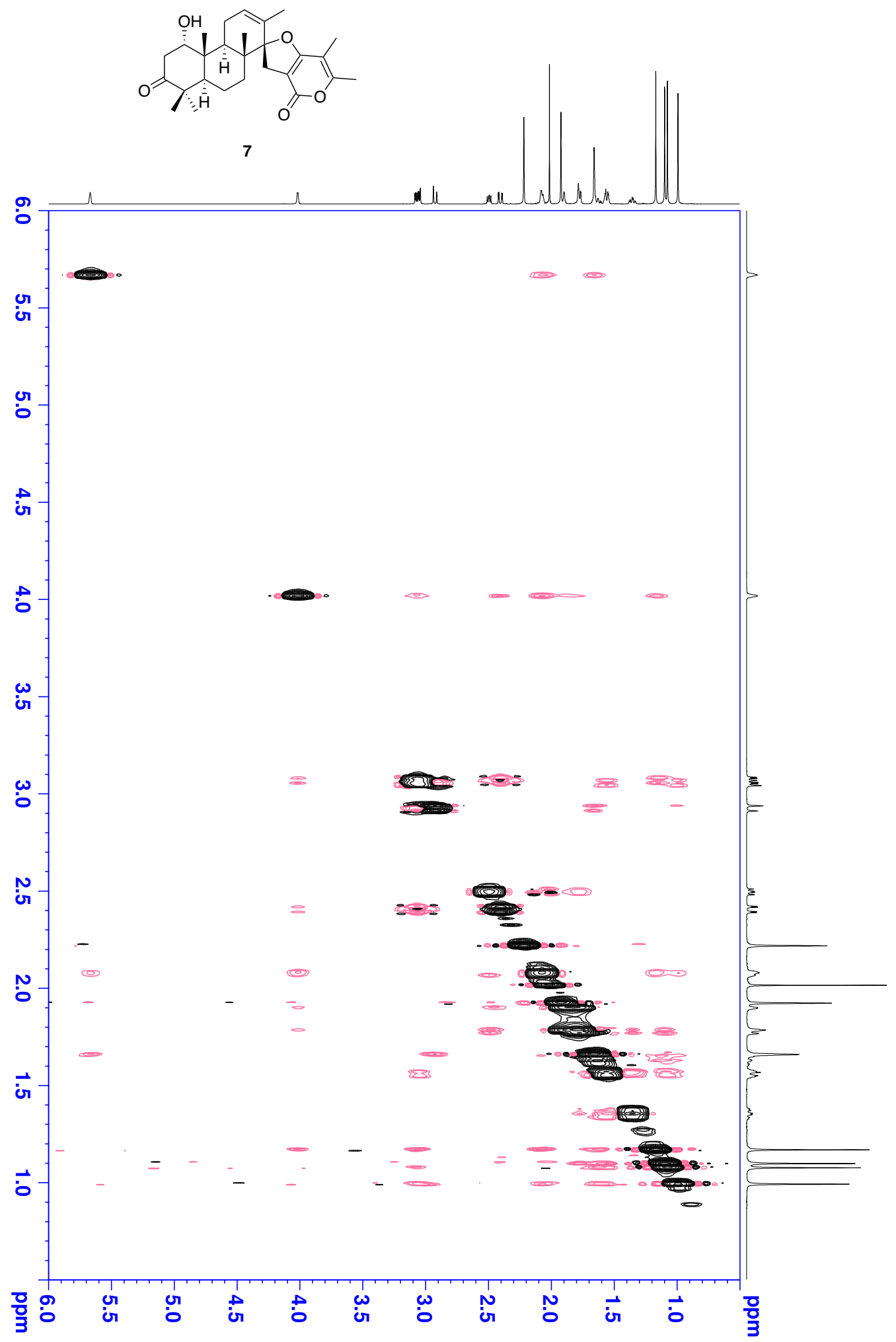

Figure S34. NOESY spectrum of brevione $\mathrm{S}(7)$ in $\mathrm{CDCl}_{3}$. 

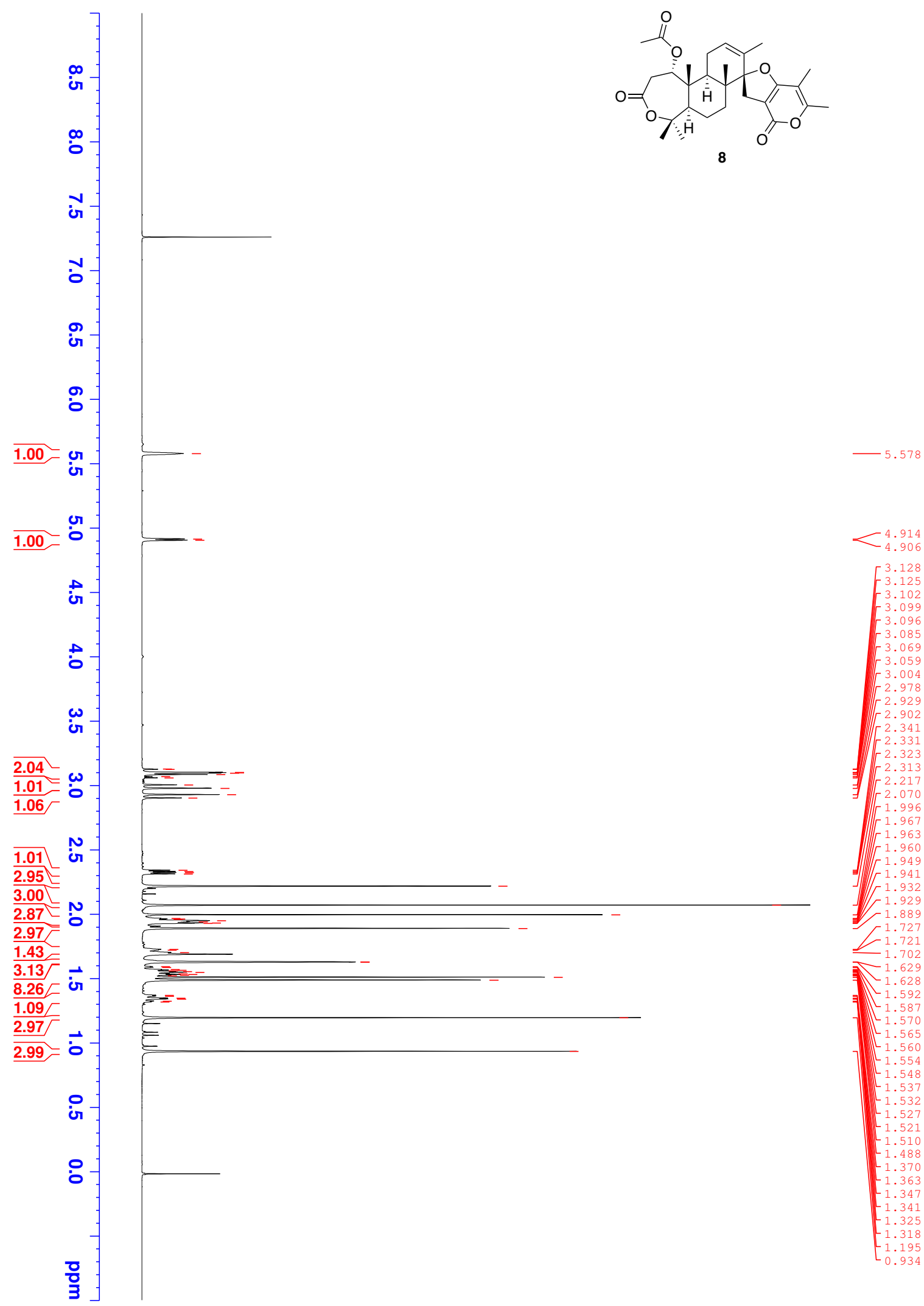

Figure S35. ${ }^{1} \mathrm{H}$ NMR spectrum of brevione $\mathrm{T}(\mathbf{8})$ in $\mathrm{CDCl}_{3}$ at $600 \mathrm{MHz}$. 


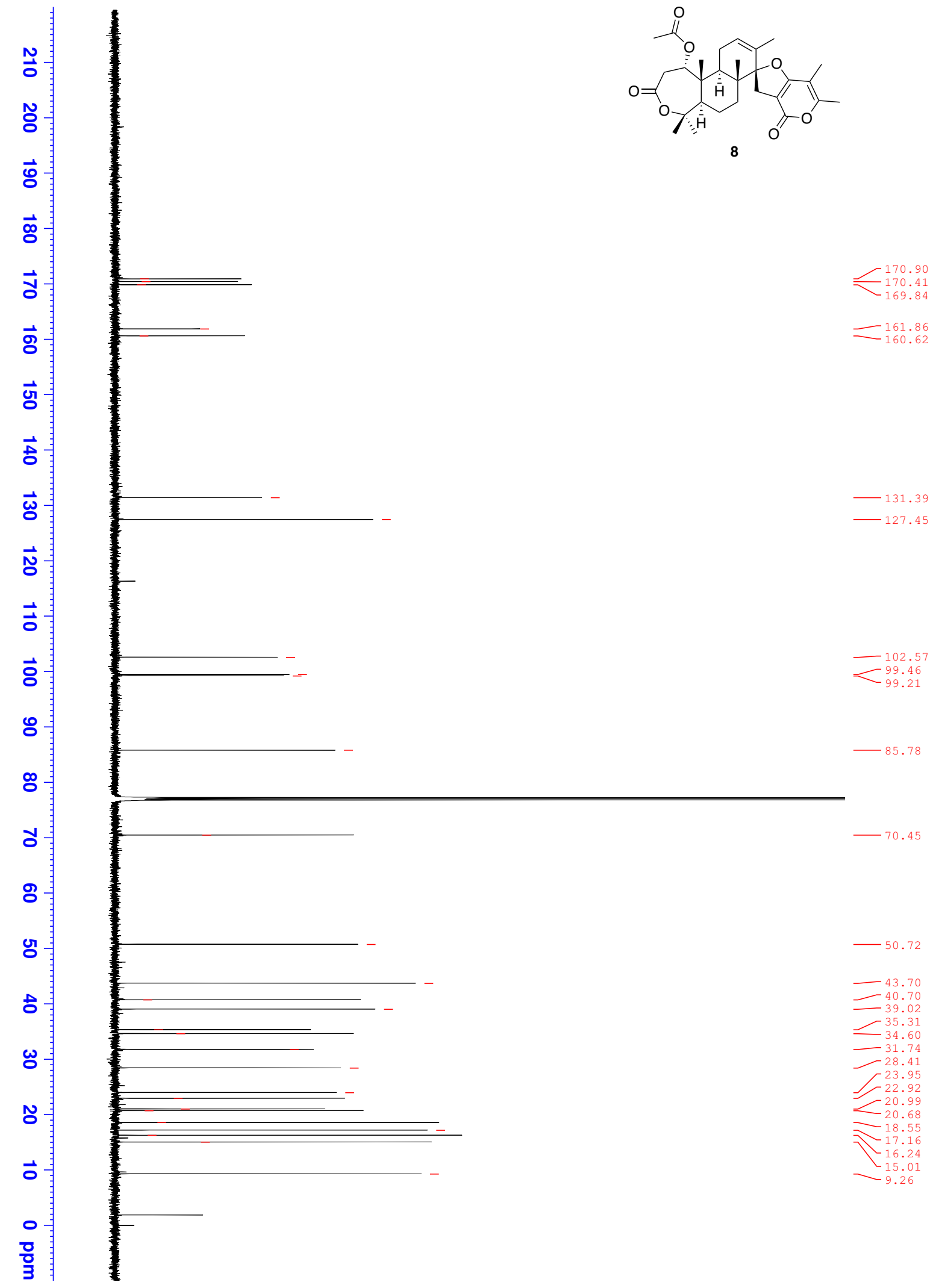

Figure S36. ${ }^{13} \mathrm{C}$ NMR spectrum of brevione $\mathrm{T}(\mathbf{8})$ in $\mathrm{CDCl}_{3}$ at $150 \mathrm{MHz}$. 


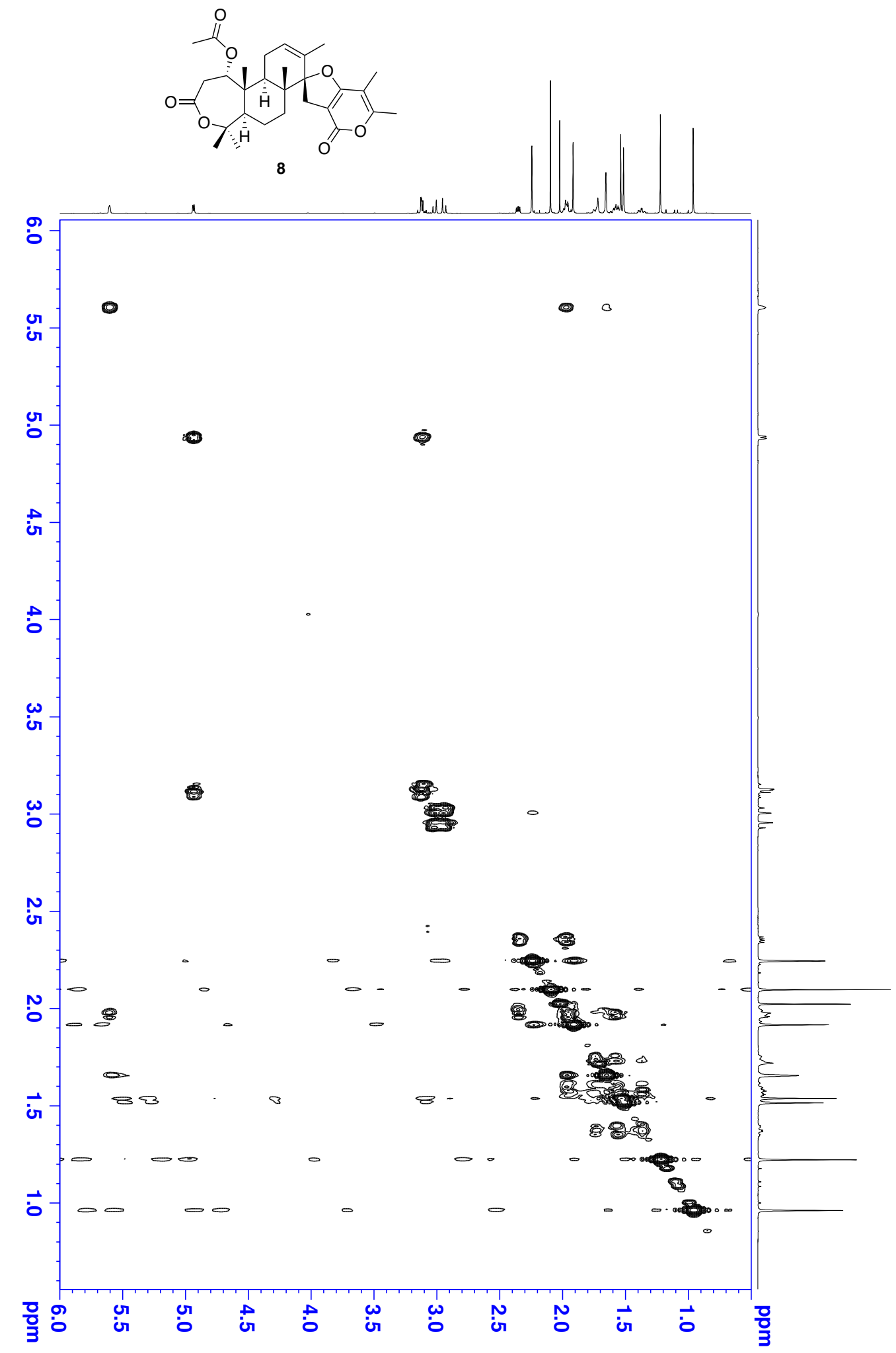

Figure $\mathrm{S} 37 .{ }^{1} \mathrm{H}-{ }^{1} \mathrm{H}$ COSY spectrum of brevione $\mathrm{T}(\mathbf{8})$ in $\mathrm{CDCl}_{3}$. 


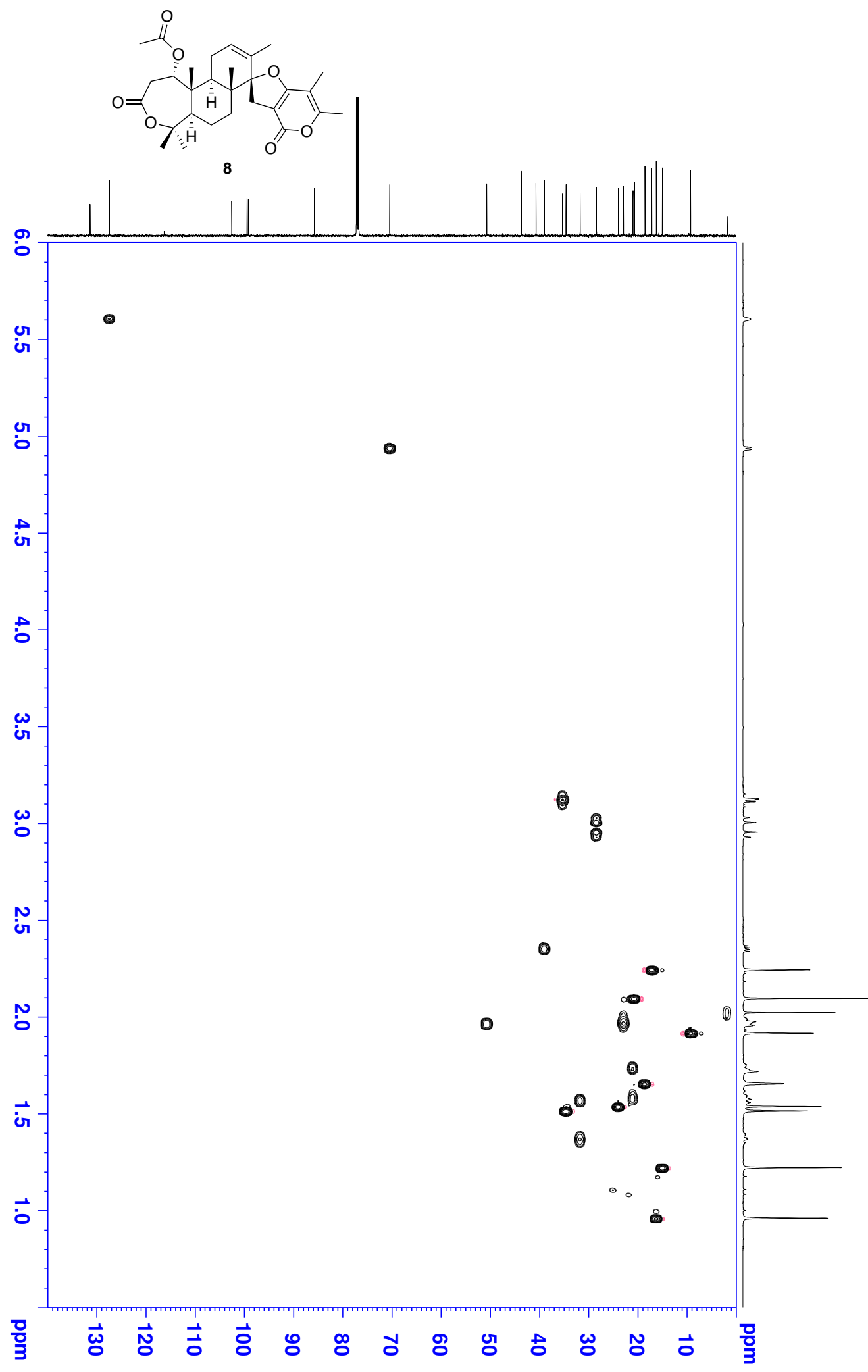

Figure S38. HSQC spectrum of brevione $\mathrm{T}(\mathbf{8})$ in $\mathrm{CDCl}_{3}$. 


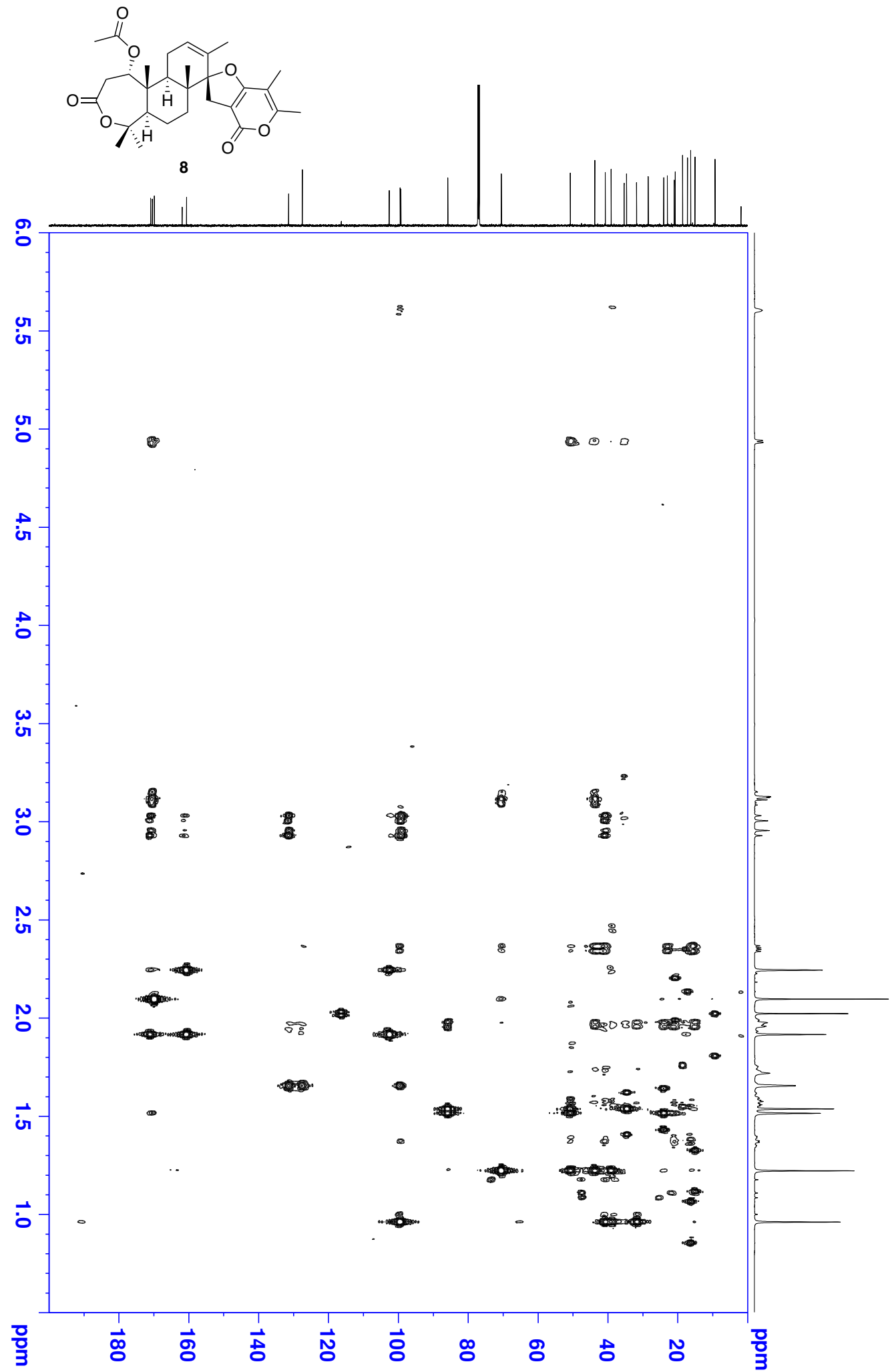

Figure S39. HMBC of brevione $\mathrm{T}(\mathbf{8})$ in $\mathrm{CDCl}_{3}$. 


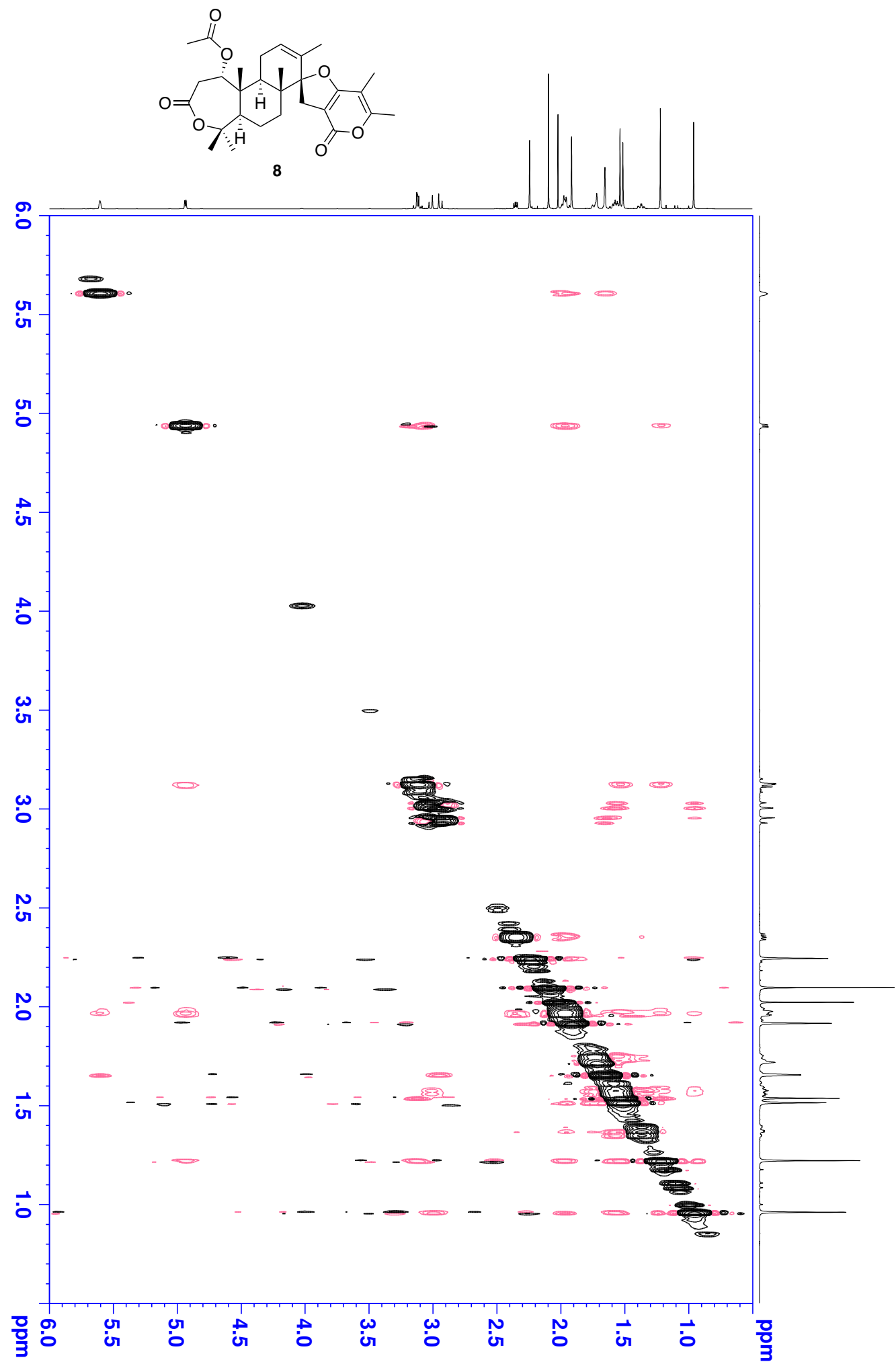

Figure S40. NOESY spectrum of brevione $\mathrm{T}(\mathbf{8})$ in $\mathrm{CDCl}_{3}$. 


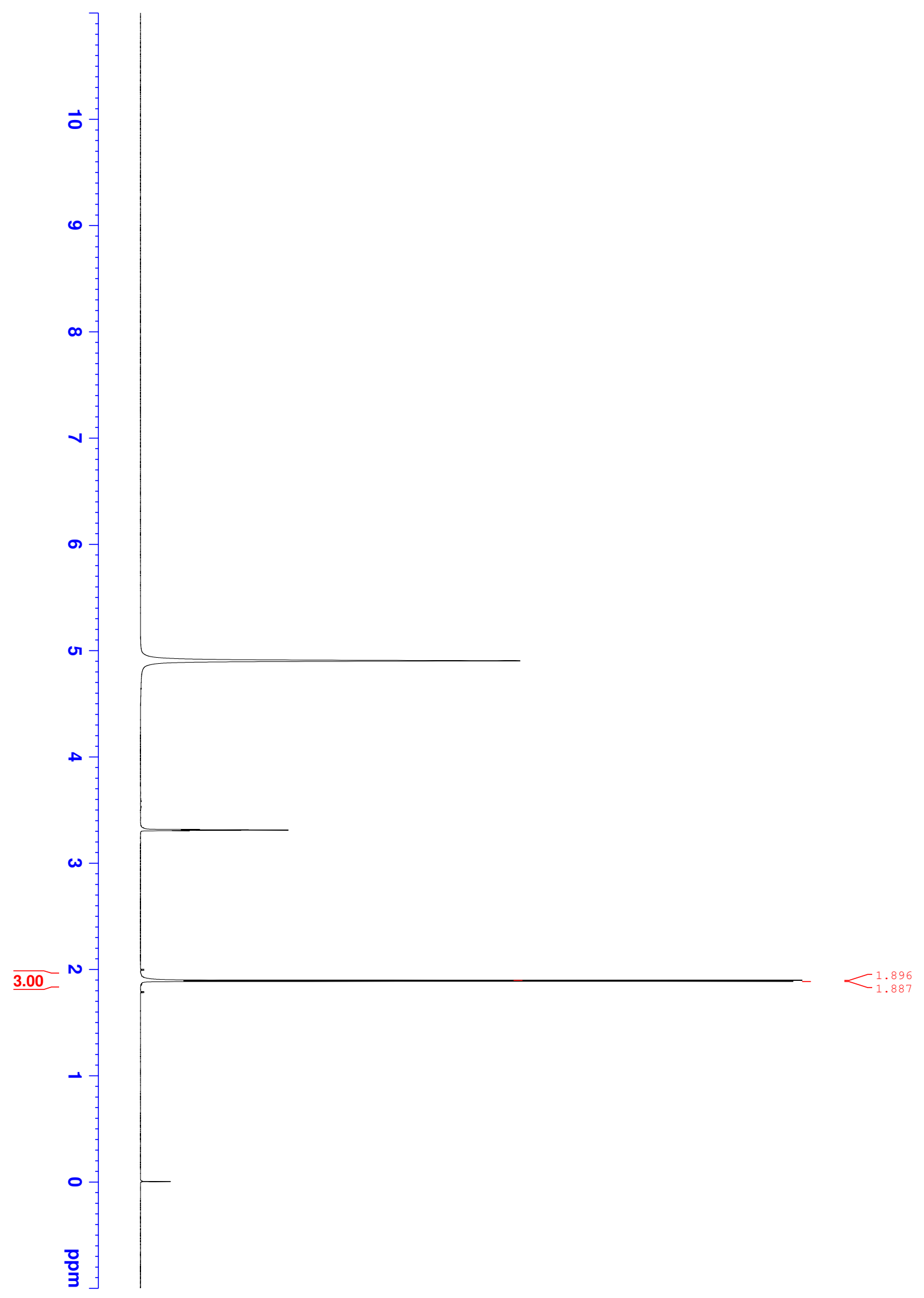

Figure S41. ${ }^{1} \mathrm{H}$ NMR spectrum of sodium $\left[1-{ }^{13} \mathrm{C},{ }^{18} \mathrm{O}_{2}\right]$ acetate in $\mathrm{CD}_{3} \mathrm{OD}$ at $600 \mathrm{MHz}$. 


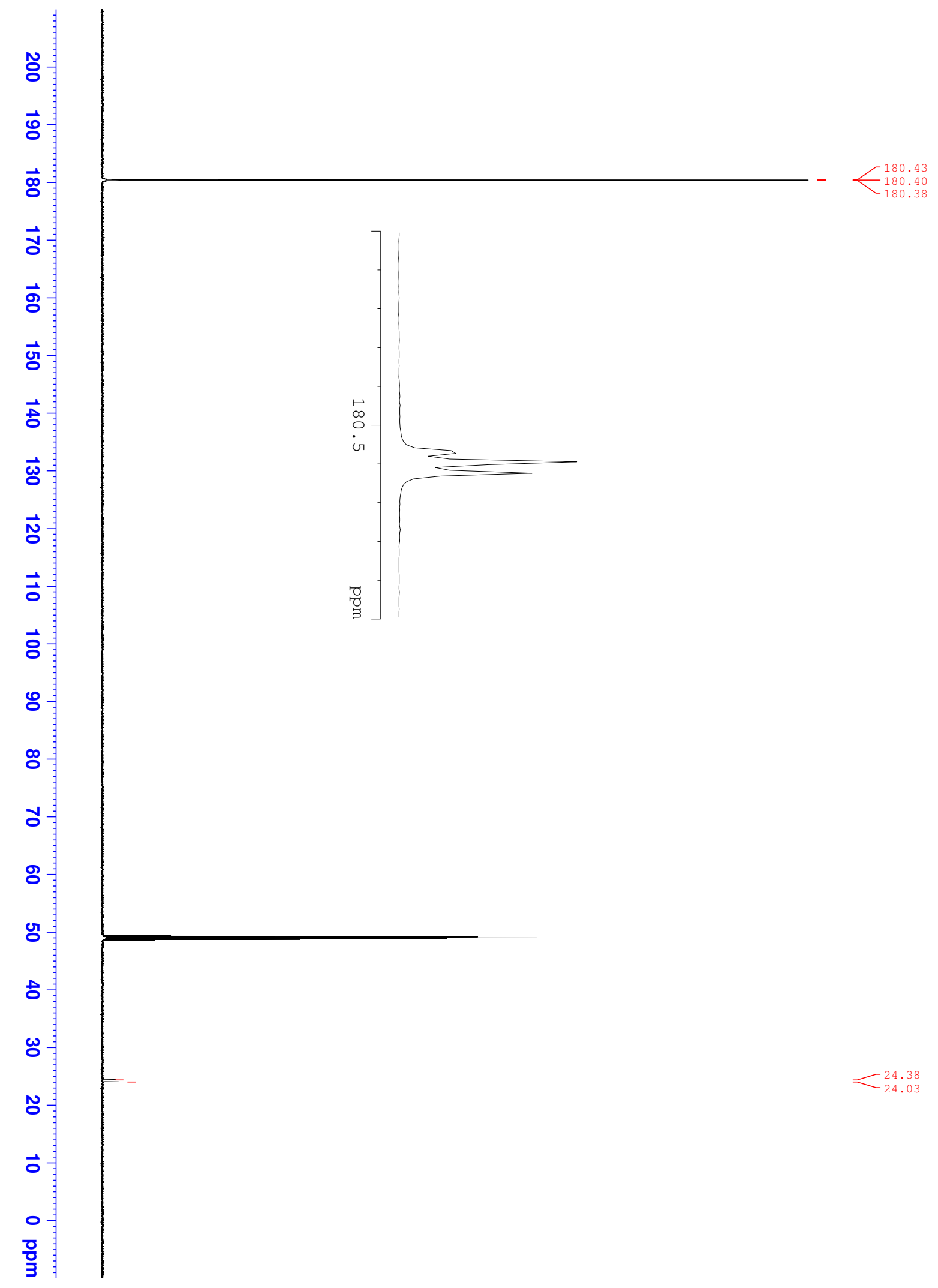

Figure $\mathrm{S} 42 .{ }^{13} \mathrm{C}$ NMR spectrum of sodium $\left[1-{ }^{13} \mathrm{C},{ }^{18} \mathrm{O}_{2}\right]$ acetate in $\mathrm{CD}_{3} \mathrm{OD}$ at $150 \mathrm{MHz}$. 


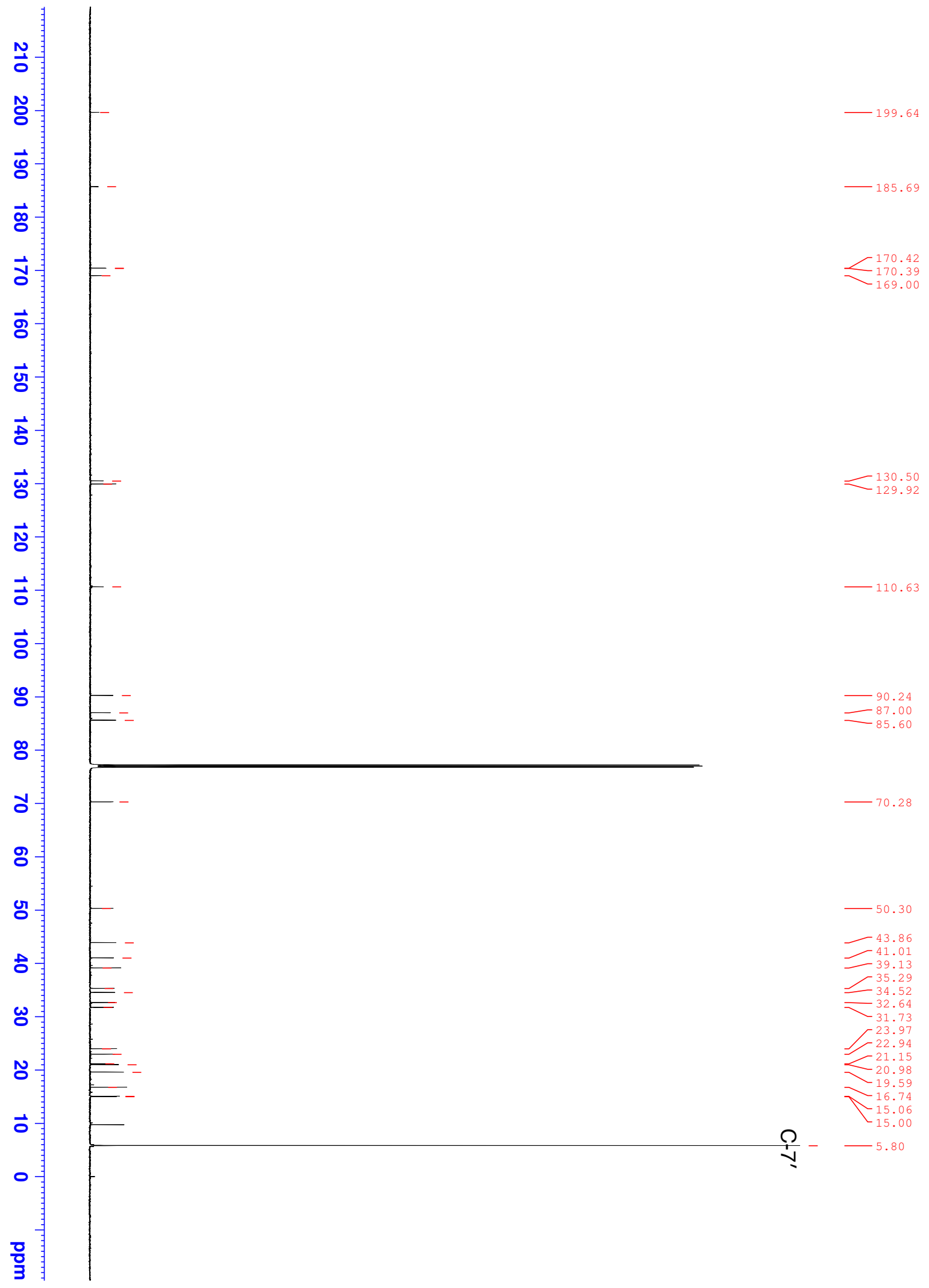

Figure S43. ${ }^{13} \mathrm{C}$ NMR spectrum of setosusin (1) labeled with $\left[\right.$ methyl $\left.-{ }^{13} \mathrm{C}\right]$ methionine in $\mathrm{CDCl}_{3}$ at $150 \mathrm{MHz}$. 


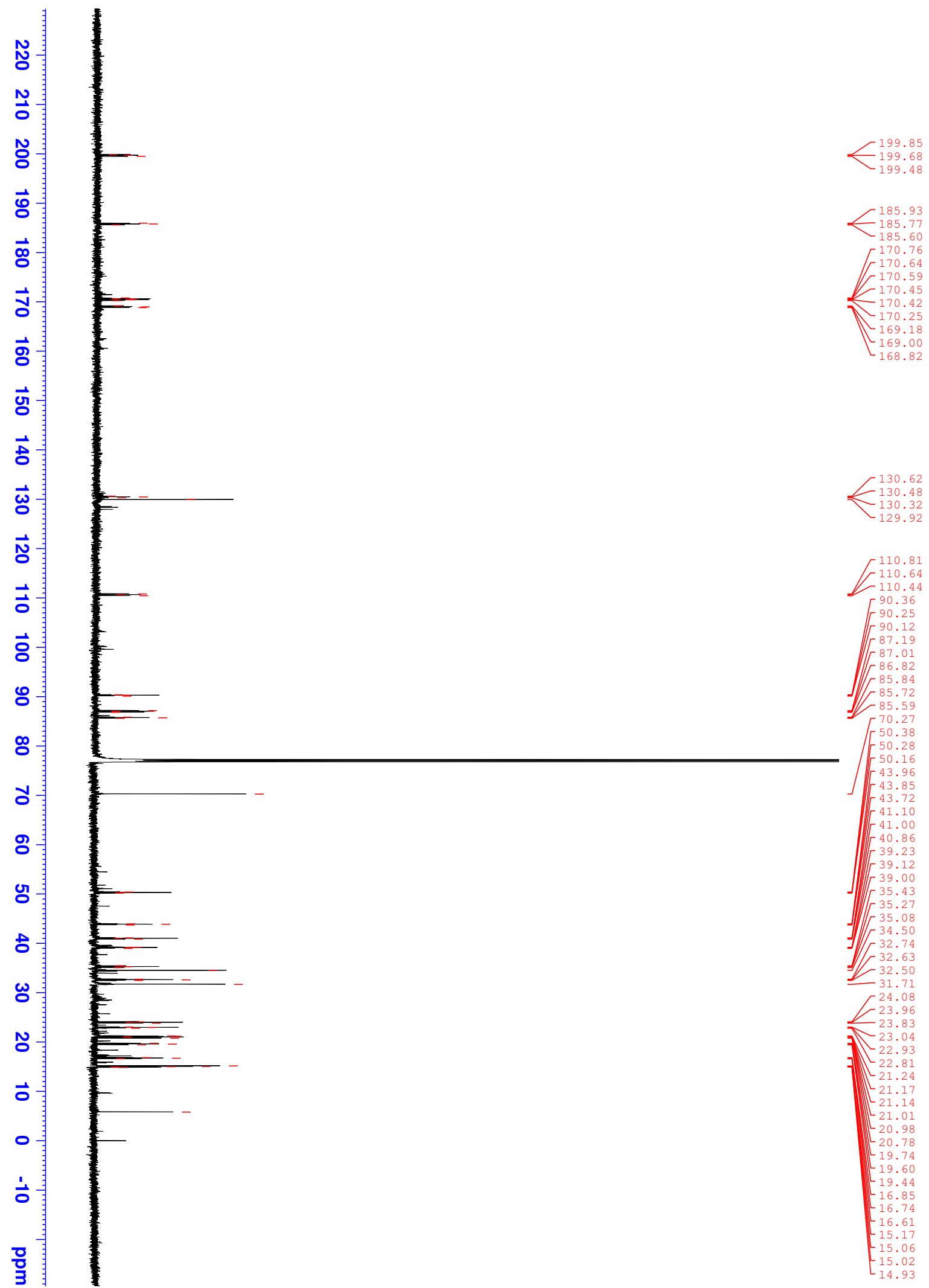

Figure S44. ${ }^{13} \mathrm{C}$ NMR spectrum of setosusin (1) labeled with sodium $\left[1,2-{ }^{13} \mathrm{C}_{2}\right]$ acetate in $\mathrm{CDCl}_{3}$ at $150 \mathrm{MHz}$. 


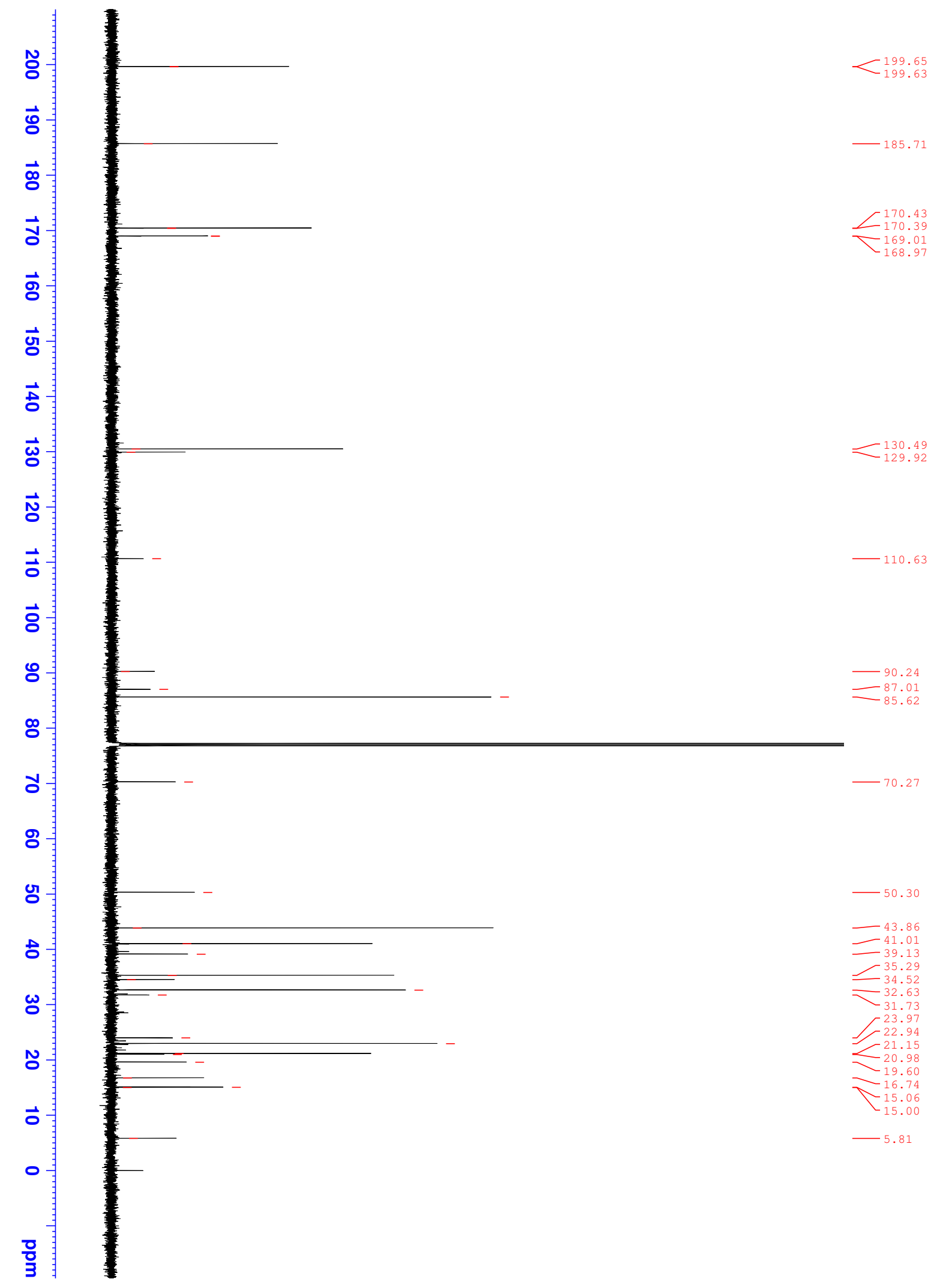

Figure $\mathrm{S} 45 .{ }^{13} \mathrm{C}$ NMR spectrum of setosusin (1) labeled with sodium $\left[1-{ }^{13} \mathrm{C},{ }^{18} \mathrm{O}_{2}\right]$ acetate in $\mathrm{CDCl}_{3}$ at $150 \mathrm{MHz}$. 


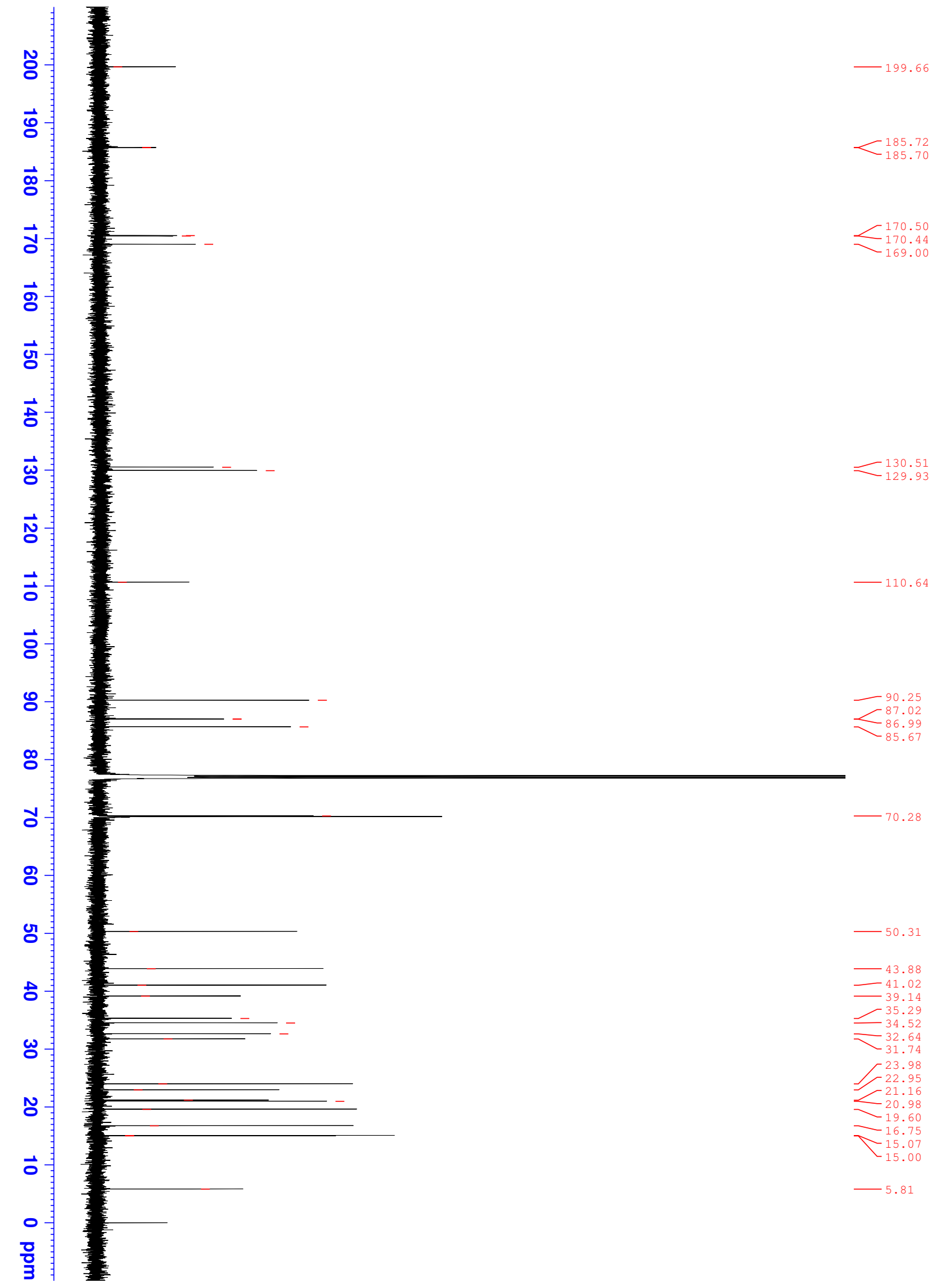

Figure S46. ${ }^{13} \mathrm{C}$ NMR spectrum of setosusin (1) labeled with ${ }^{18} \mathrm{O}_{2}$ in $\mathrm{CDCl}_{3}$ at $150 \mathrm{MHz}$. 

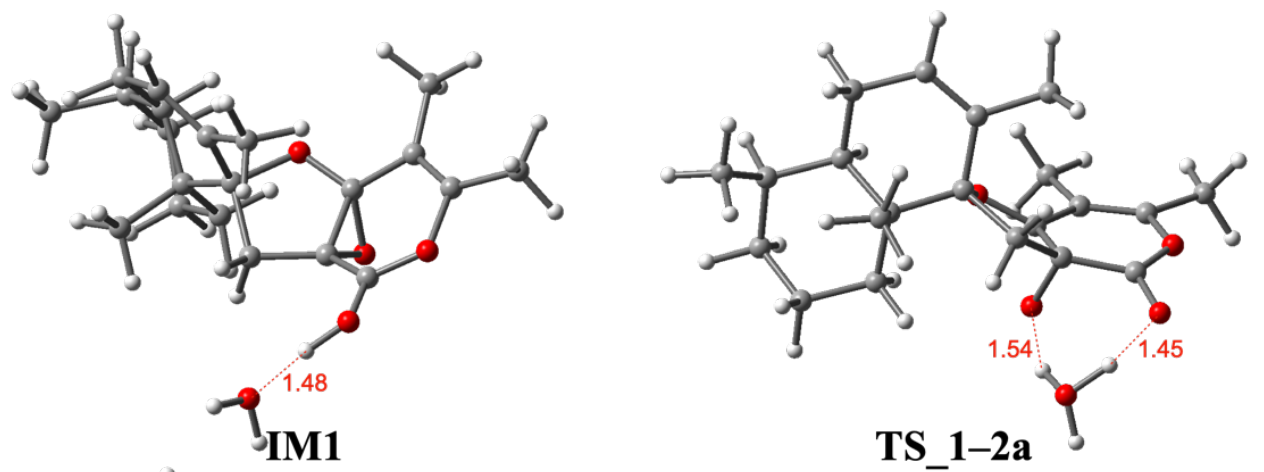

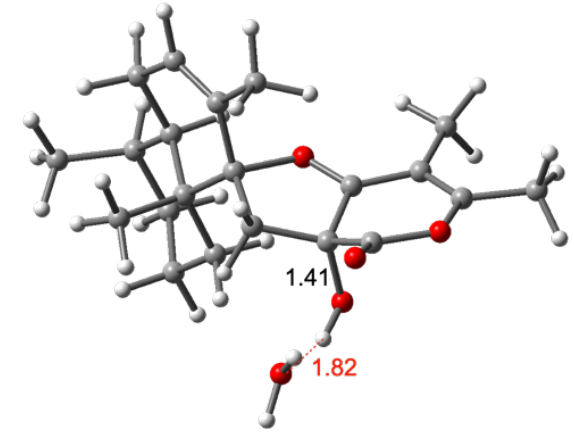

IM2a

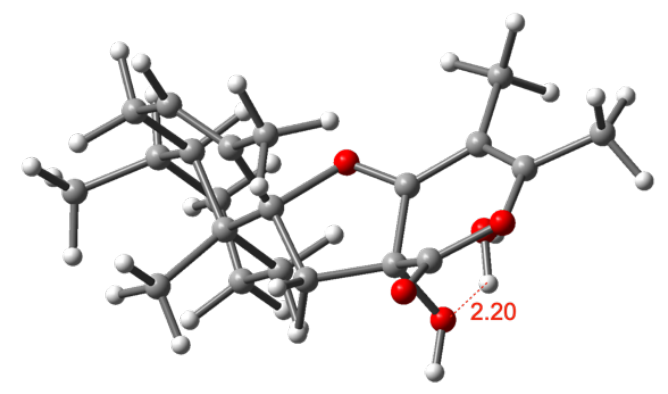

IM2b

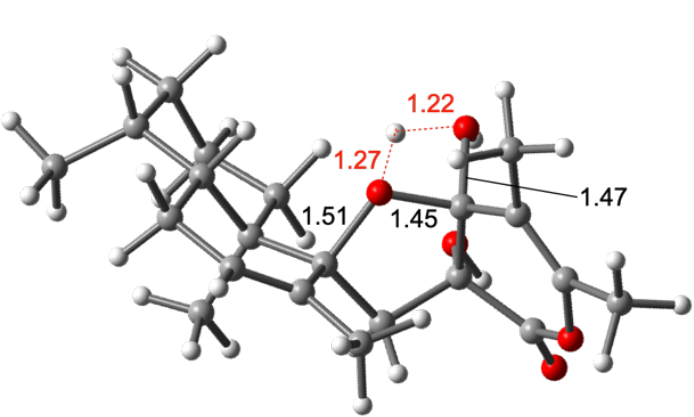

TS_2b-3

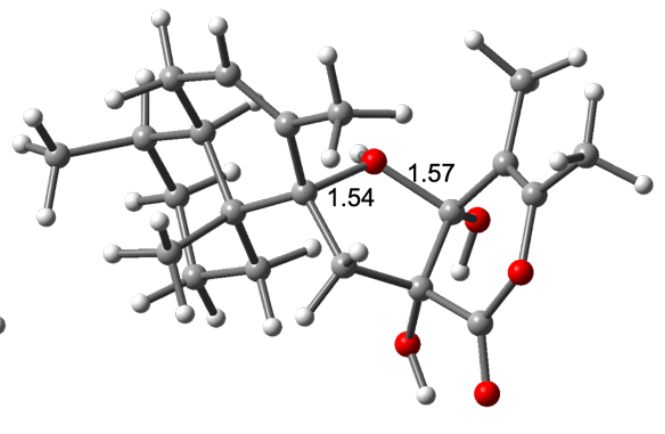

IM3

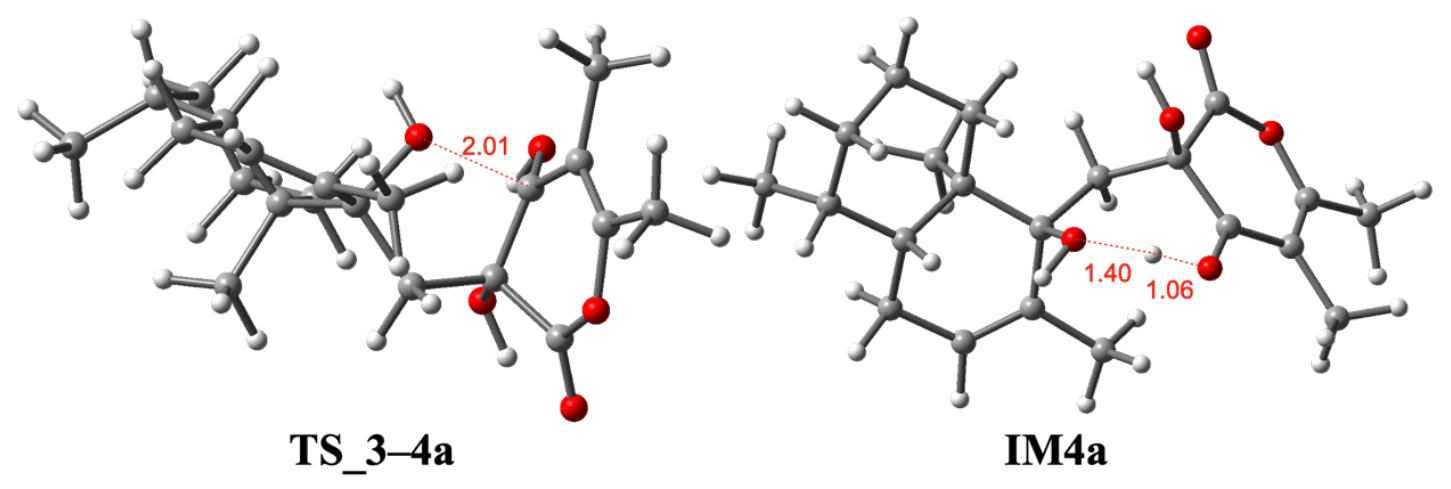

(to be continued to the next page) 


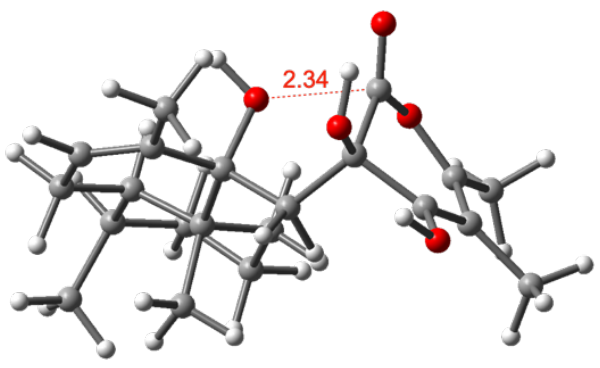

IM4b

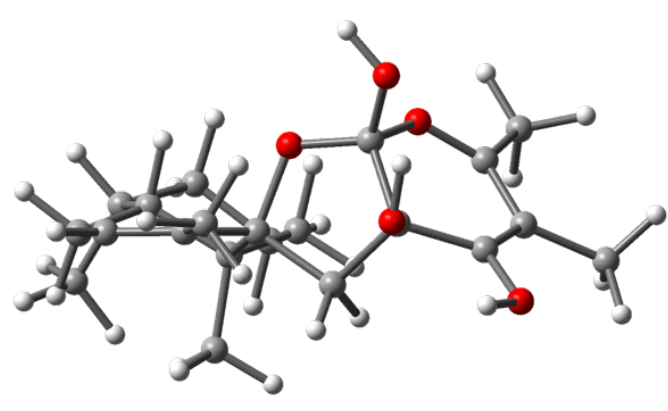

IM5

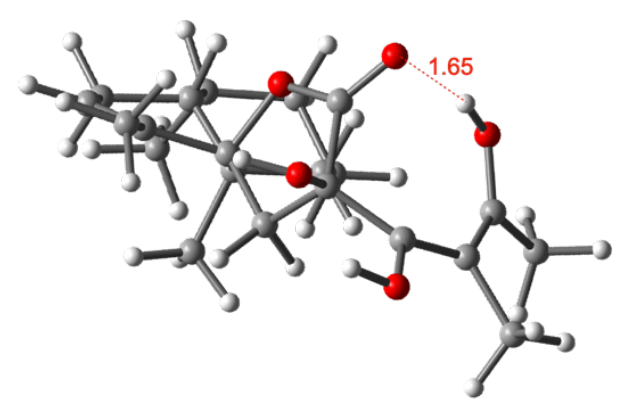

IM6a

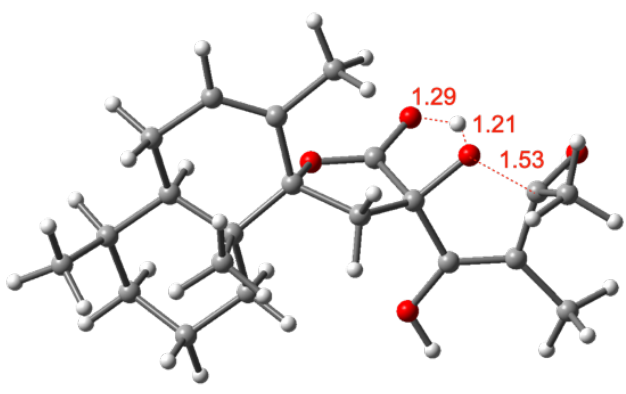

TS_6b-7

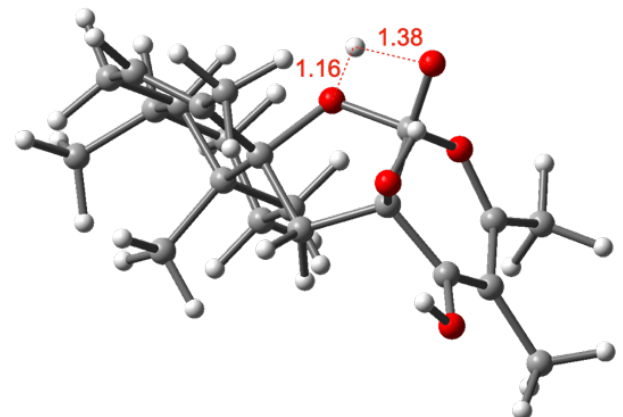

TS_4b-5

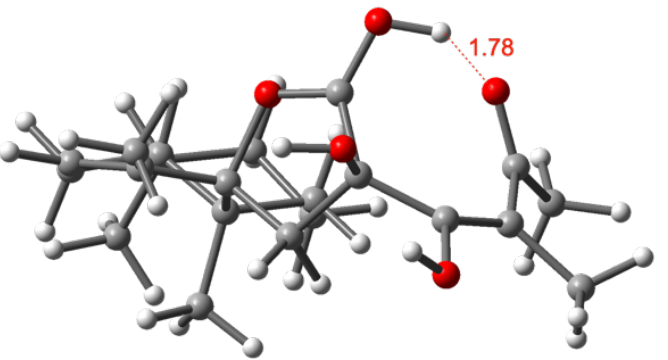

TS_5-6a

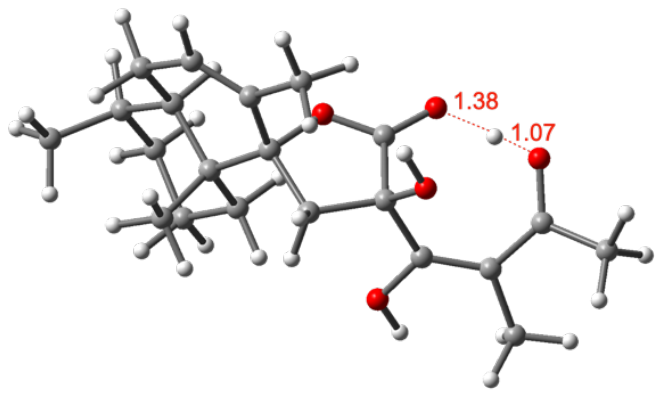

IM6b

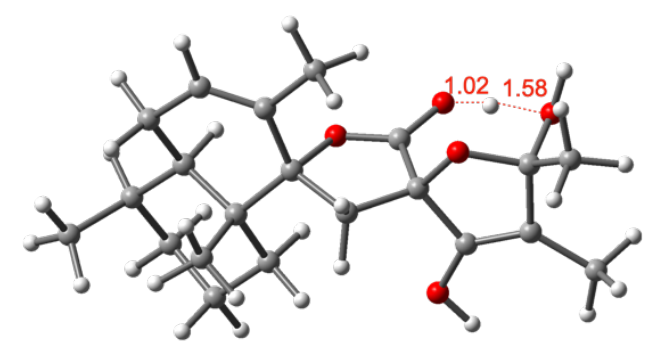

IM7

(to be continued to the next page) 


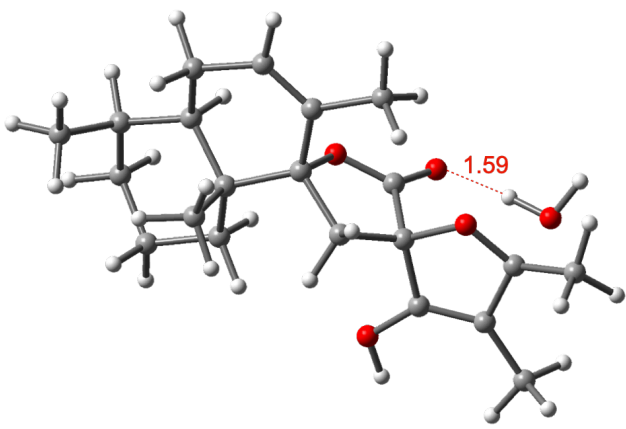

TS_7-8

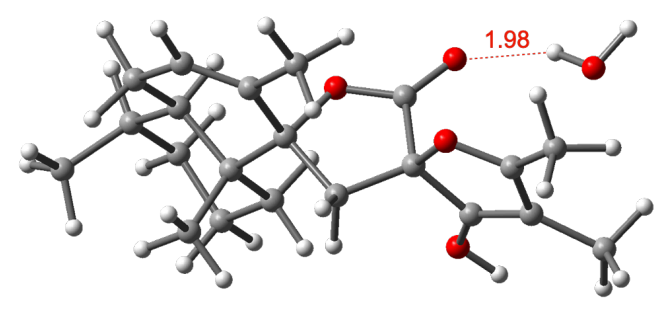

IM8

Figure S47. 3D representation of IM1, TS_1-2a, IM2a, IM2b, TS_2b-3, IM3, TS_3-4a, IM4a, IM4b, TS_4b-5, IM5, TS_5-6a, IM6a, IM6b, TS_6b-7, IM7, TS_7-8, and IM8. Selected distances are shown in $\AA$. 

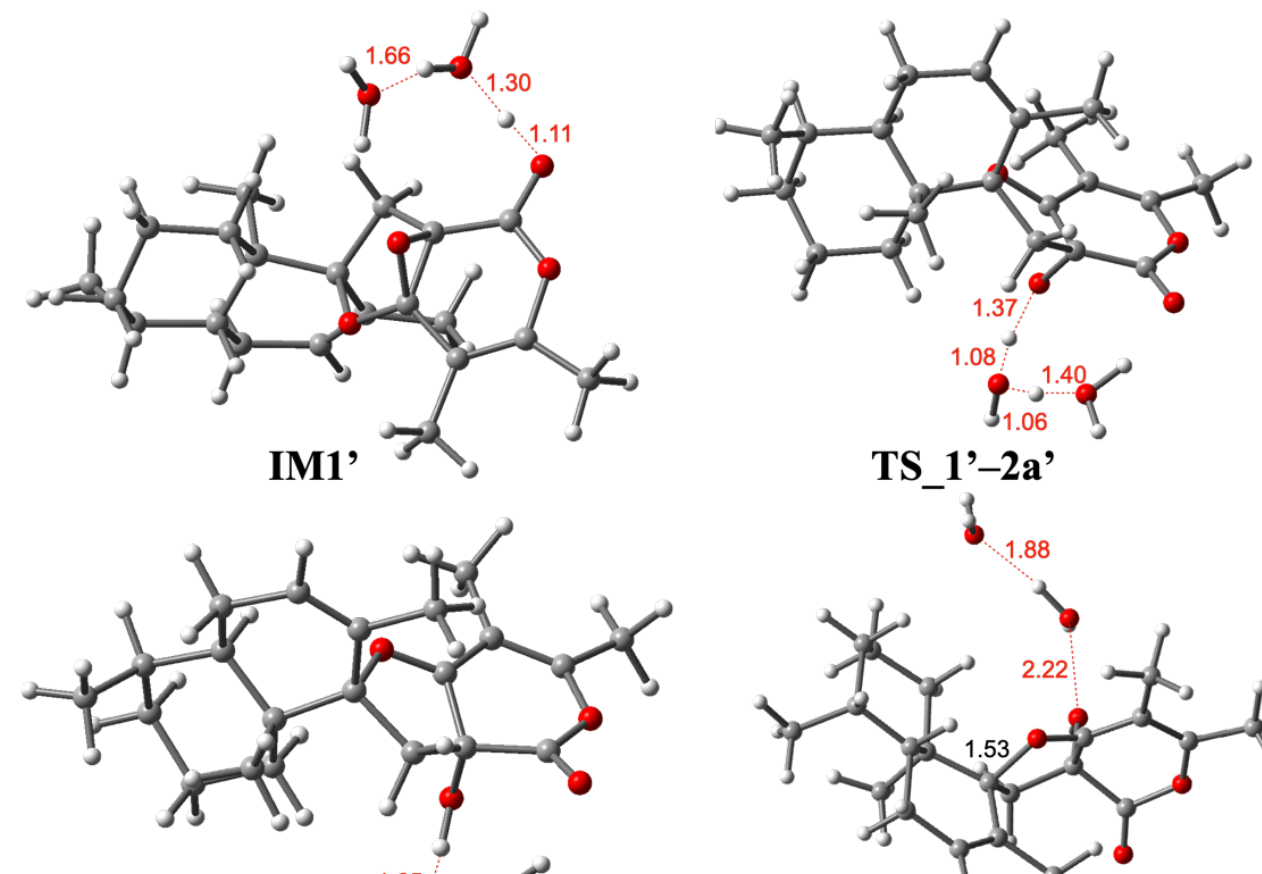

$$
\text { IM2a }
$$
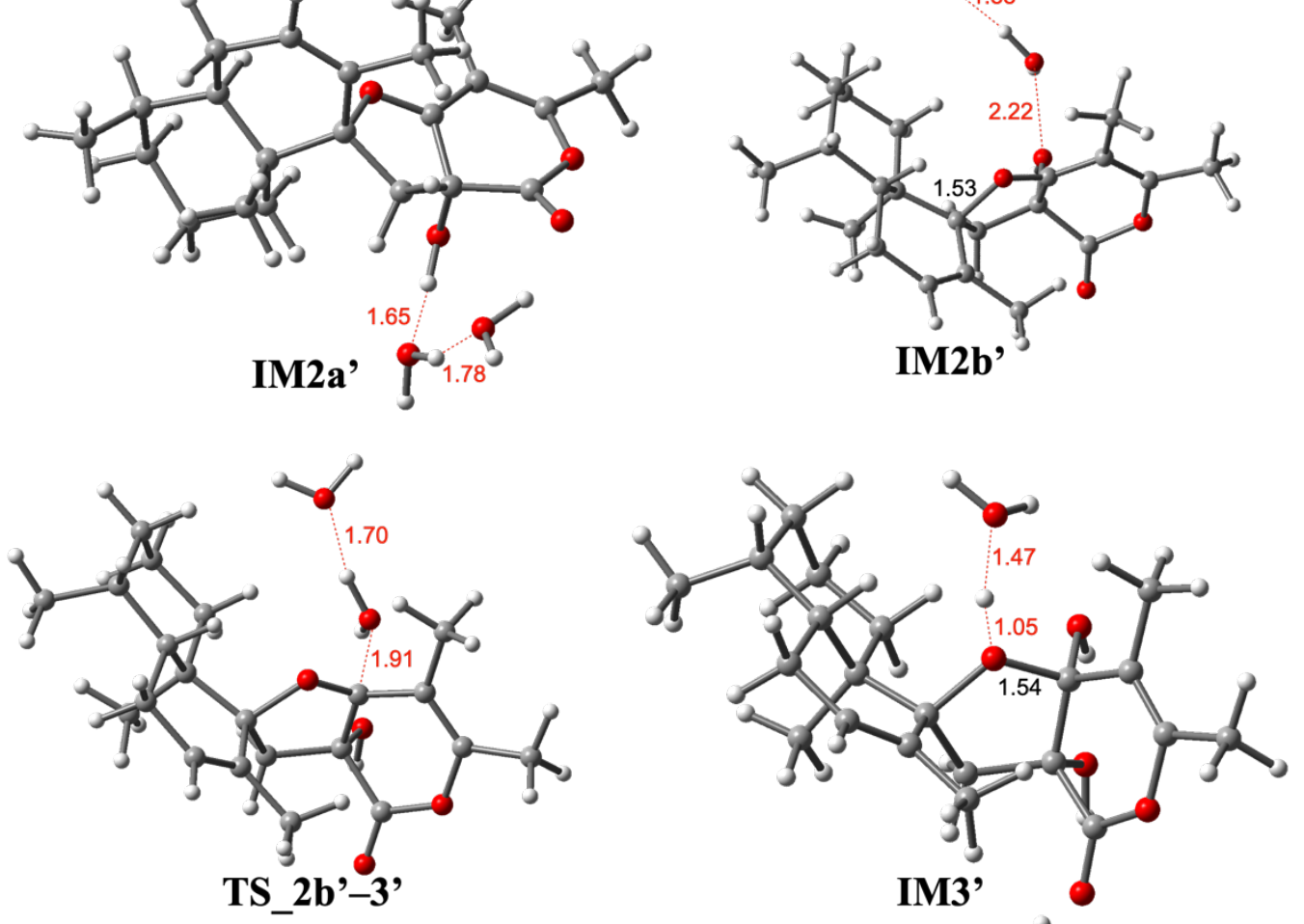

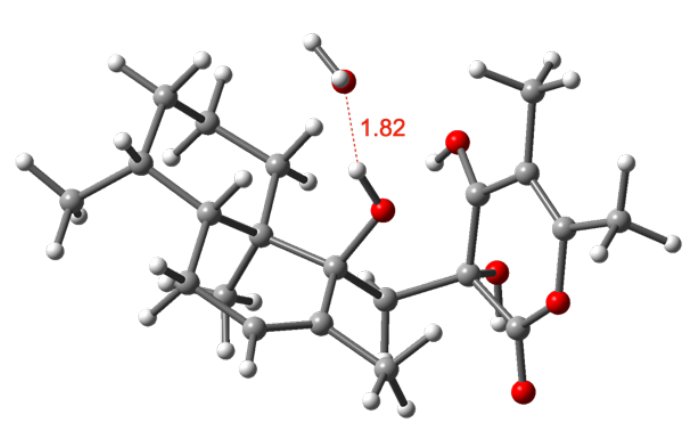

TS_3'-4a'

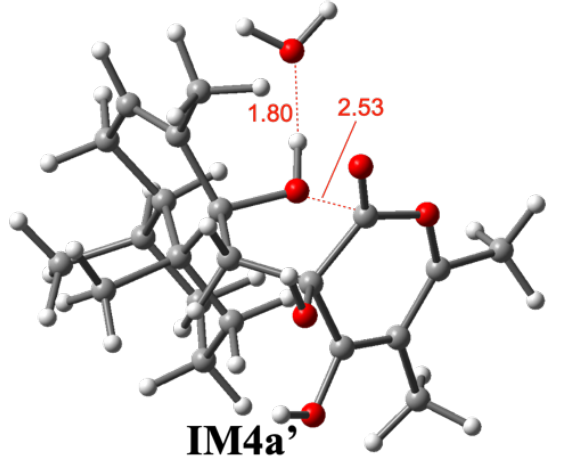

(to be continued to the next page) 

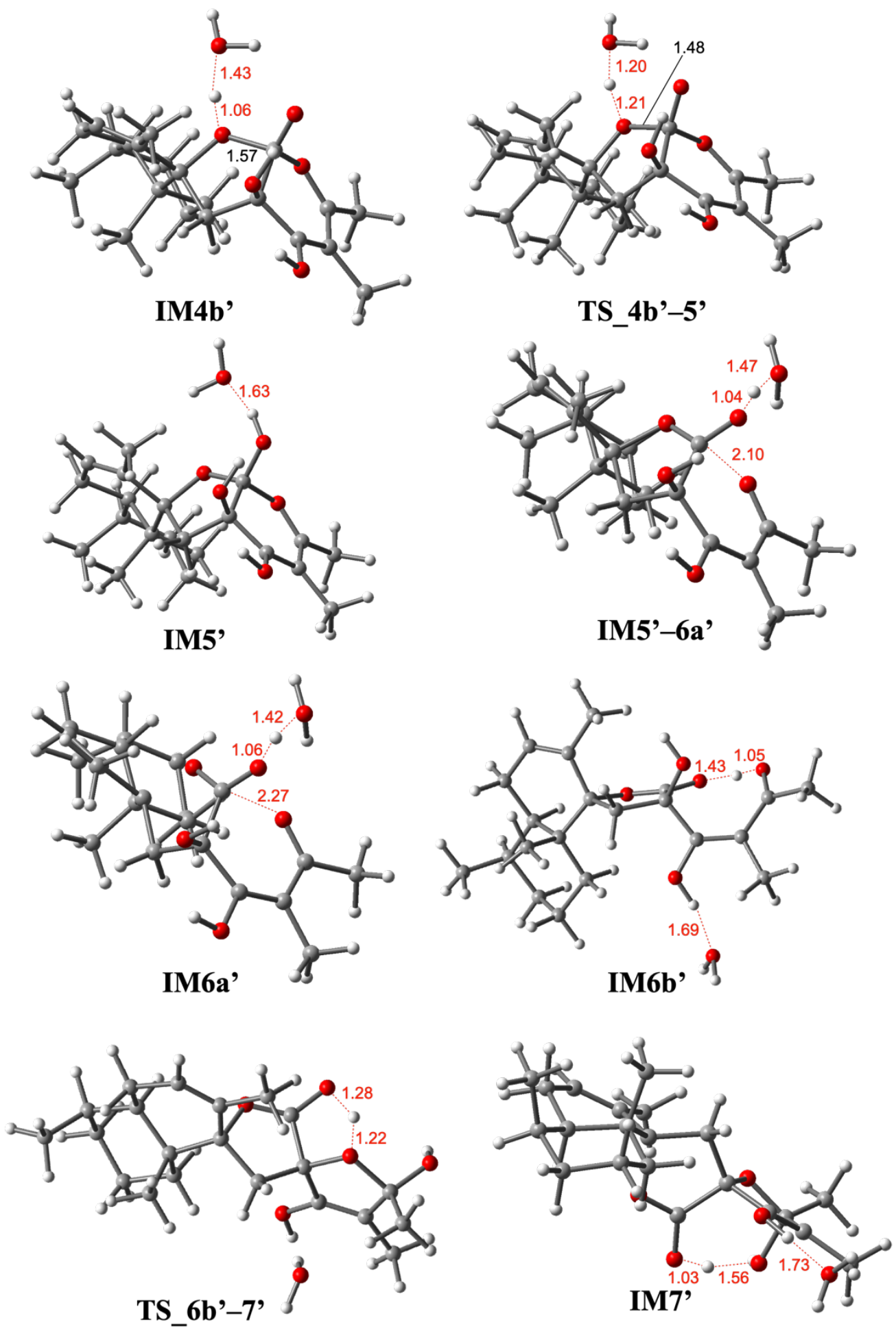

(to be continued to the next page) 


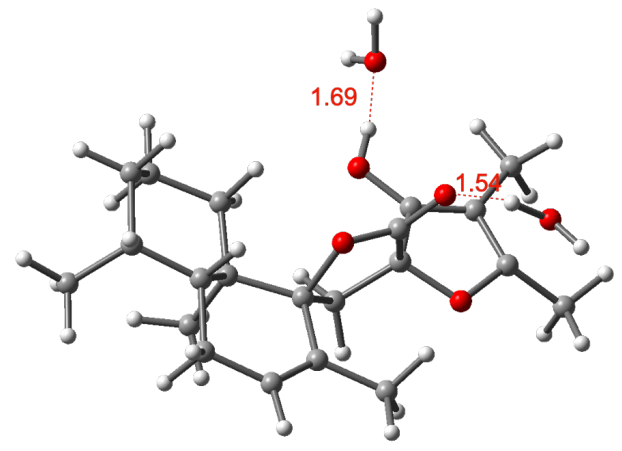

TS_7'-8'

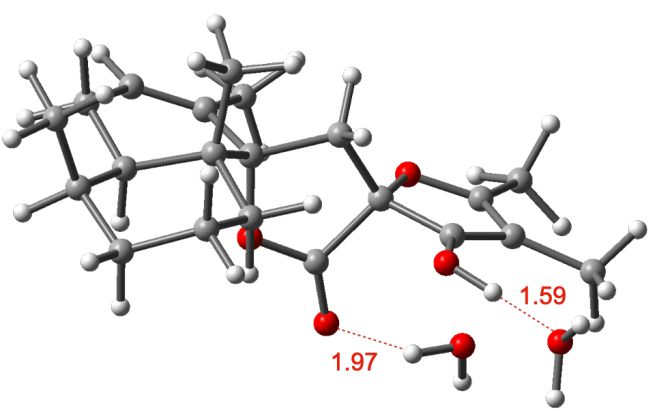

IM8

Figure S48. 3D representation of IM1', TS_1'-2a', IM2a', IM2b', TS_2b'-3', IM3', TS_3'-4a', IM4a', IM4b', TS_4b'-5', IM5', TS_5'-6a', IM6a', IM6b', TS_6b'-7', IM7', TS_7'-8', and IM8'. Selected distances are shown in $\AA$. 


\section{Cartesian Coordinates and Energies}

IM1

Sum of electronic and thermal Free Energies $=-1193.862560$

\begin{tabular}{|c|c|c|c|}
\hline C & 2.81443500 & -0.65295400 & 0.53703600 \\
\hline $\mathbf{H}$ & 2.26849500 & -1.60218800 & 0.42076300 \\
\hline C & 1.93700100 & 0.15459600 & -1.69816200 \\
\hline H & 1.39555500 & -0.77450700 & -1.90746900 \\
\hline H & 1.39824900 & 0.95668400 & -2.21835800 \\
\hline C & 3.34931100 & 0.02199200 & -2.27013300 \\
\hline H & 3.90772200 & 0.95772400 & -2.14317600 \\
\hline $\mathbf{H}$ & 3.28416300 & -0.15088200 & -3.34883800 \\
\hline C & 5.33065900 & 0.08114300 & 0.22923000 \\
\hline H & 5.39827200 & 0.32400500 & 1.29288000 \\
\hline H & 6.28870200 & -0.36066300 & -0.06013200 \\
\hline $\mathbf{H}$ & 5.23685600 & 1.01743500 & -0.32686700 \\
\hline C & 2.41031300 & 1.84301600 & 0.12717400 \\
\hline H & 1.92119800 & 2.57145600 & -0.52729800 \\
\hline H & 2.21206500 & 2.13101800 & 1.16411100 \\
\hline H & 3.48217200 & 1.94033500 & -0.03957600 \\
\hline C & 1.93303300 & 0.40745700 & -0.17607100 \\
\hline C & 0.49071100 & 0.26208000 & 0.38542100 \\
\hline C & 1.51622700 & -0.14433700 & 2.61919300 \\
\hline H & 1.41421800 & -0.24126400 & 3.69924400 \\
\hline C & 2.87879800 & -0.36406500 & 2.03617100 \\
\hline $\mathbf{H}$ & 3.36397800 & -1.19916200 & 2.55603000 \\
\hline H & 3.50142100 & 0.51642100 & 2.24407400 \\
\hline C & 0.42397300 & 0.13833800 & 1.90151500 \\
\hline C & -0.46528100 & 1.38901300 & -0.14109000 \\
\hline $\mathbf{H}$ & -0.65827200 & 2.13185300 & 0.63421400 \\
\hline $\mathbf{H}$ & -0.04892900 & 1.90023300 & -1.00965600 \\
\hline 0 & -0.09929300 & -1.00957600 & -0.11096800 \\
\hline C & -1.69827500 & 0.63788600 & -0.56326200 \\
\hline C & -1.362958 & -0.8 & -0.53890100 \\
\hline C & -3.09224300 & 0.924 & -0.25235100 \\
\hline C & -2.39319200 & -1.84 & -0.2 \\
\hline C & -3.66194100 & -1.41657000 & -0.19810600 \\
\hline 0 & -3.96232300 & -0.02706200 & -0.26083800 \\
\hline 0 & -3.55155900 & 2.06435300 & 0.07688900 \\
\hline C & -4.93704700 & -2.16595200 & -0.03614500 \\
\hline H & -5.59962400 & -1.95397000 & -0.87995200 \\
\hline H & -4.75249200 & -3.23786200 & -0.00498800 \\
\hline $\mathbf{H}$ & -5.44772900 & -1.86311400 & 0.88198200 \\
\hline C & -1.93320600 & -3.25262600 & -0.03533500 \\
\hline $\mathbf{H}$ & -2.74974400 & -3.91451500 & 0.24727200 \\
\hline H & -1.46443800 & -3.63695200 & -0.94617400 \\
\hline H & -1.17603600 & -3.27067500 & 0.75339900 \\
\hline C & 4.21016700 & -0.92230900 & -0.08183700 \\
\hline H & 4.52989300 & -1.87404700 & 0.36451600 \\
\hline C & 4.08259100 & -1.13797700 & -1.59635500 \\
\hline H & 3.52218500 & -2.06296500 & -1.78825500 \\
\hline $\mathbf{H}$ & 5.07734500 & -1.27355700 & -2.03452400 \\
\hline C & -0.93983300 & 0.20875800 & 2.54600700 \\
\hline H & -1.44111000 & 1.17344800 & 2.39920500 \\
\hline H & -1.60331800 & -0.57442100 & 2.15403000 \\
\hline H & -0.86042800 & 0.05050800 & 3.62304900 \\
\hline 0 & -1.52632200 & -0.12664000 & -1.75632000 \\
\hline 0 & -2.16127900 & 4.10236400 & -0.38372400 \\
\hline $\mathbf{H}$ & -2.09655200 & 4.77981600 & 0.30302500 \\
\hline
\end{tabular}

H $\quad-2.93606600 \quad 2.87390400 \quad-0.09631100$

H $\quad \mathbf{- 2 . 3 2 0 9 9 1 0 0} \quad \mathbf{4 . 5 5 8 4 9 3 0 0} \quad \mathbf{- 1 . 2 2 1 0 9 9 0 0}$
IM2a

Sum of electronic and thermal Free Energies $=-1193.902915$

\begin{tabular}{|c|c|c|c|}
\hline C & -2.81167800 & -0.79691700 & -0.16663200 \\
\hline & -2.21964500 & -1.65245700 & 0.19427800 \\
\hline & -1.61245200 & 0.73907800 & 1.45466600 \\
\hline & -1.01072100 & -0.06698500 & 1.89388800 \\
\hline & -1.02421800 & 1.65891900 & 1.55468300 \\
\hline & -2.90458500 & 0.84607100 & 2.26628100 \\
\hline & -3.51066400 & 1.68882500 & 1.91241300 \\
\hline & -2.65525700 & 1.06537200 & 3.30884700 \\
\hline & -5.27302000 & 0.05351200 & 0.27454700 \\
\hline & -5.51055100 & -0.07904400 & -0.78413600 \\
\hline & -6.15670200 & -0.25353900 & 0.84123300 \\
\hline & -5.13819900 & 1.12194300 & 0.46069600 \\
\hline & -2.46349700 & 1.67939000 & 3544500 \\
\hline & -1.92061800 & 2.58720000 & -0.45714200 \\
\hline & -2.43666400 & 1.58959800 & -1.82529100 \\
\hline 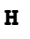 & -3.50199300 & 1.82874700 & -0.44466700 \\
\hline & -1.87662900 & 0.43505100 & -0.03443500 \\
\hline & -0.54960900 & 0.10023800 & -0.75947500 \\
\hline & -1.88632400 & -1.08209100 & -2.48041100 \\
\hline & -1.95788700 & -1.54783400 & -3.46216900 \\
\hline & -3.12310500 & -1.07078900 & -1.63679900 \\
\hline 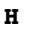 & -3.64049200 & -2.03236300 & -1.73748300 \\
\hline[ & -3.81319800 & -0.31926600 & -2.04365700 \\
\hline 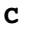 & -0.71021800 & -0.55544300 & -2.11725800 \\
\hline : & 0.50201200 & 1.24048700 & -0.73840300 \\
\hline $\mathbf{H}$ & 0.82431600 & 1.51035700 & -1.74446700 \\
\hline 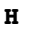 & 0.11482000 & 2.14135300 & -0.26593900 \\
\hline 0 & 0.15345400 & -1.01910600 & 0.03507000 \\
\hline C & 1.67358300 & 0.72777800 & 0.09641200 \\
\hline C & 1.36885400 & -0.73657500 & 0.27790200 \\
\hline C & 3.07225900 & 0.89294200 & -0.47360600 \\
\hline C & 2.347545 & -1.642019 & 0.70588900 \\
\hline C & 3.643944 & -1.17417800 & 0.61237200 \\
\hline 0 & 3.99677900 & 0.00550900 & 0.062367 \\
\hline 0 & 3.45398200 & 1.75064500 & -1.20369100 \\
\hline C & 4.84447800 & -1.94758300 & 1.01309500 \\
\hline H & 5.53198100 & -1.28554900 & 1.54622100 \\
\hline H & 4.58653300 & -2.80177900 & 1.63563200 \\
\hline $\mathbf{H}$ & 5.36409600 & -2.29594800 & 0.11265600 \\
\hline C & 2.00395700 & -3.03995100 & 1.15330600 \\
\hline H & 2.59824300 & -3.78886300 & 0.62604500 \\
\hline $\mathbf{H}$ & 2.16568300 & -3.15800600 & 2.22817300 \\
\hline $\mathbf{H}$ & 0.95138700 & -3.24009500 & 0.94874700 \\
\hline C & -4.07698200 & -0.79609300 & 0.72947700 \\
\hline $\mathbf{H}$ & -4.42592800 & -1.83759900 & 0.70984300 \\
\hline $\mathbf{C}$ & -3.69375500 & -0.46074800 & 2.17804400 \\
\hline H & -3.07607500 & -1.27043700 & 2.59024700 \\
\hline $\mathbf{n}$ & -4.59710000 & -0.40790900 & 2.79489600 \\
\hline & 0.51414300 & -0.65847400 & -2.99238600 \\
\hline & 0.77767900 & 0.29107200 & -3.46909100 \\
\hline
\end{tabular}




$\begin{array}{lrrr}\text { H } & 1.39546700 & -0.99193000 & -2.42424600 \\ \text { H } & 0.35315400 & -1.39132000 & -3.78451700 \\ \text { O } & 1.73909400 & 1.23739100 & 1.40988800 \\ \text { O } & 2.07756500 & 3.75115800 & 0.39070800 \\ \text { H } & 2.06124800 & 4.63632300 & 0.77281700 \\ \text { H } & 2.71904900 & 3.76546600 & -0.33240400 \\ \text { H } & 1.85174500 & 2.20886000 & 1.32631100\end{array}$

IM2b

Sum of electronic and thermal Free Energies $=-1193.893747$

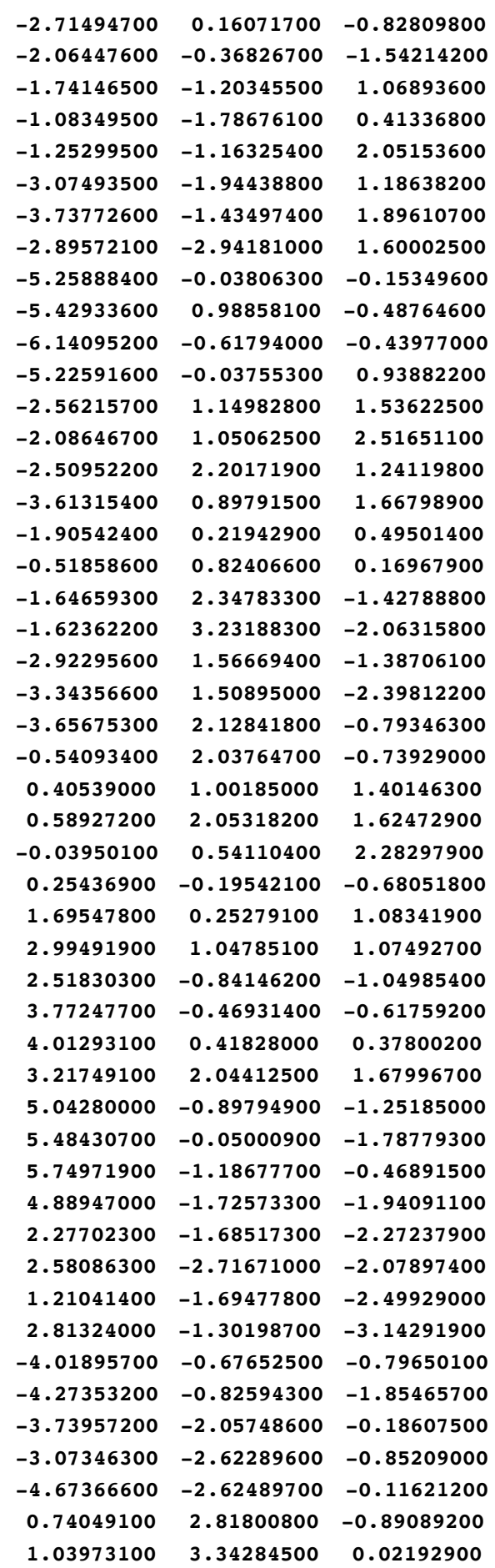

$\begin{array}{lrrr}\text { H } & 1.57919800 & 2.16787900 & -1.18443700 \\ \text { H } & 0.63839100 & 3.56344500 & -1.68105800 \\ \text { O } & 1.94038500 & -0.87476000 & 1.91437900 \\ \text { H } & 2.04356200 & -0.57743100 & 2.83084500 \\ \text { C } & 1.46114700 & -0.30728900 & -0.30001200 \\ \text { O } & 1.12552300 & -3.02565100 & 0.23244700 \\ \text { H } & 1.39939200 & -2.86313400 & 1.14405200 \\ \text { H } & 0.72501300 & -3.90192400 & 0.21689700\end{array}$

IM3

Sum of electronic and thermal Free Energies $=-1193.878408$

\begin{tabular}{|c|c|c|c|}
\hline $\mathbf{C}$ & 2.69042000 & 0.75381500 & 0.41465800 \\
\hline H & 2.16072100 & 0.85501500 & 1.38068200 \\
\hline C & 1.80395200 & -1.59854400 & 0.03291600 \\
\hline H & 1.25275000 & -1.68136700 & 0.98478000 \\
\hline H & 1.27658500 & -2.24815800 & -0.66940100 \\
\hline 工 & 3.21178200 & -2.13310600 & 0.30163100 \\
\hline $\mathbf{H}$ & 3.78204800 & -2.18265900 & -0.63305800 \\
\hline $\mathbf{H}$ & 3.13547400 & -3.16145000 & 0.66662000 \\
\hline $\mathbf{C}$ & 5.21315400 & 0.28682000 & -0.20574300 \\
\hline H & 5.30933200 & 1.28791100 & -0.63271900 \\
\hline $\mathbf{H}$ & 6.15764100 & 0.06401500 & 0.29839800 \\
\hline H & 5.12117100 & -0.42643400 & -1.02821400 \\
\hline C & 2.29382500 & -0.14081400 & -1.95367600 \\
\hline H & 1.82846900 & -0.94277200 & -2.53278800 \\
\hline H & 2.08983800 & 0.80732800 & -2.45705600 \\
\hline H & 3.36895300 & -0.30873700 & -1.99059100 \\
\hline 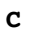 & 1.79966700 & -0.14644700 & -0.49038800 \\
\hline C & 0.35762100 & 0.46309800 & -0.49939200 \\
\hline C & 1.44398900 & 2.69472700 & -0.56798200 \\
\hline H & 1.38182400 & 3.76581400 & -0.75138100 \\
\hline C & 2.77978700 & 2.16095100 & -0.16483300 \\
\hline H & 3.24691200 & 2.83861500 & 0.55942500 \\
\hline H & 3.43291100 & 2.17501000 & -1.04765400 \\
\hline 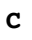 & 0.33497100 & 1.96544200 & -0.74596600 \\
\hline 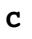 & -0.68227800 & -0.33427900 & -1.32411000 \\
\hline H & -1.33126200 & 0.35236200 & -1.87008900 \\
\hline H & -0.20402100 & -0.98473800 & -2.05683200 \\
\hline 0 & -0.24807700 & 0.31519600 & 0.91155200 \\
\hline C & -1.54300700 & -1.22483100 & -0.39440200 \\
\hline C & -2.94834700 & -1.34923600 & -0.98279400 \\
\hline C & -2.64560800 & 0.48357500 & 1.15767300 \\
\hline C & -3.64323400 & 0.55984100 & 0.26511700 \\
\hline 0 & -3.78262900 & -0.31289900 & -0.81226300 \\
\hline 0 & -3.25303800 & -2.30974500 & -1.62946200 \\
\hline C & -4.77639400 & 1.52672000 & 0.24012200 \\
\hline H & -4.81385000 & 2.00314800 & -0.74420300 \\
\hline H & -5.72149300 & 0.99484400 & 0.38279000 \\
\hline H & -4.67428600 & 2.29668200 & 1.00141500 \\
\hline 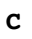 & -2.51243200 & 1.36823700 & 2.36894900 \\
\hline 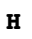 & -2.14387100 & 0.78100300 & 3.21451300 \\
\hline H & -1.80324500 & 2.18304800 & 2.18576200 \\
\hline & -3.46733700 & 1.79916200 & 2.66504000 \\
\hline & 4.07630900 & 0.18427900 & 0.82157600 \\
\hline & 4.38643800 & 0.80720800 & 1.67131700 \\
\hline & 3.92759300 & -1.25626500 & 1.32632000 \\
\hline & 3.34992300 & -1.26306900 & 2.26217100 \\
\hline & 4.91330700 & -1.66702100 & 1.56653200 \\
\hline & -0.95886100 & 2.63117100 & -1.14712000 \\
\hline
\end{tabular}




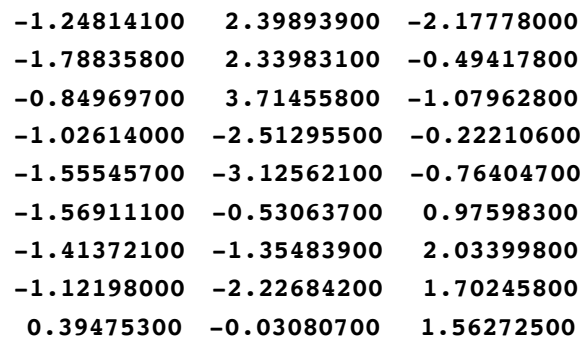

IM4a

Sum of electronic and thermal Free Energies $=-1193.889629$

\begin{tabular}{|c|c|c|c|}
\hline C & 3.13744200 & 0.76611200 & 0.19869900 \\
\hline & 2.89083100 & 1.04368600 & 1.23855600 \\
\hline C & 2.13473200 & -1.54275700 & 0.50633100 \\
\hline H & 1.82924100 & -1.38418000 & 1.54695200 \\
\hline $\mathbf{H}$ & 1.44452000 & -2.28183100 & 0.07662900 \\
\hline C & 3.55052600 & -2.12287100 & 0.49859000 \\
\hline $\mathbf{H}$ & 3.85212800 & -2.38051300 & -0.52398900 \\
\hline $\mathbf{H}$ & 3.55683600 & -3.05911700 & 1.06470600 \\
\hline C & 5.39564700 & 0.02429800 & -0.95993700 \\
\hline $\mathbf{H}$ & 5.35626400 & 0.90583300 & -1.60504600 \\
\hline $\mathbf{H}$ & 6.44388700 & -0.12365200 & -0.68531000 \\
\hline $\mathbf{H}$ & 5.10205000 & -0.84539900 & -1.55285200 \\
\hline C & 2.14138300 & -0.47327800 & -1.78945900 \\
\hline $\mathbf{H}$ & 1.60567400 & -1.37832900 & -2.08981000 \\
\hline 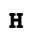 & 1.75541500 & 0.36221800 & -2.38105800 \\
\hline H & 3.18103900 & -0.62062000 & -2.07782900 \\
\hline C & 2.02658100 & -0.21299300 & -0.27083400 \\
\hline C & 0.63729200 & 0.46515700 & 0.02462900 \\
\hline C & 1.69339200 & 2.59554400 & -0.73062500 \\
\hline $\mathbf{H}$ & 1.59703300 & 3.63021100 & -1.05613900 \\
\hline C & 3.08396000 & 2.05028800 & -0.62428700 \\
\hline $\mathbf{H}$ & 3.74219400 & 2.80866200 & -0.18357500 \\
\hline $\mathbf{H}$ & 3.46720800 & 1.87961100 & -1.63890200 \\
\hline C & 0.57151400 & 1.91450200 & -0.46104300 \\
\hline C & -0.50326300 & -0.41035600 & -0.58365900 \\
\hline $\mathbf{n}$ & -0.92686200 & 0.09718900 & -1.45627700 \\
\hline $\mathbf{H}$ & -0.07646700 & -1.34096800 & -0.96199600 \\
\hline 0 & 0.43351700 & 0.50508000 & 1.48179900 \\
\hline C & -1.66035500 & -0.91421000 & 0.33050200 \\
\hline C & -2.51647200 & -1.80928000 & -0.56680000 \\
\hline C & -3.75961700 & 0.54117800 & 0.46781700 \\
\hline C & -4.26801700 & -0.19445200 & -0.58036500 \\
\hline 0 & -3.67112000 & -1.27758900 & -1.10812500 \\
\hline 0 & -2.19058800 & -2.91225400 & -0.86319200 \\
\hline C & -5.56773700 & 0.08560900 & -1.24049500 \\
\hline $\mathbf{H}$ & -5.55595700 & -0.29216200 & -2.26332400 \\
\hline $\mathbf{H}$ & -6.35922000 & -0.44388100 & -0.69419400 \\
\hline $\mathbf{H}$ & -5.79944500 & 1.15062100 & -1.22603200 \\
\hline C & -4.51213700 & 1.70628100 & 1.05890200 \\
\hline $\mathbf{H}$ & -4.08106400 & 1.97377600 & 2.02350000 \\
\hline $\mathbf{H}$ & -4.45630200 & 2.58488600 & 0.40850800 \\
\hline H & -5.56346800 & 1.45832100 & 1.21632900 \\
\hline 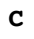 & 4.57186600 & 0.18645500 & 0.32638000 \\
\hline $\mathbf{n}$ & 5.11074900 & 0.92659100 & 0.93342900 \\
\hline$c$ & 4.53412300 & -1.12907700 & 1.11550100 \\
\hline $\mathbf{n}$ & 4.22817000 & -0.92908400 & 2.15159200 \\
\hline $\mathbf{H}$ & 5.53984100 & -1.55972700 & 1.16151500 \\
\hline
\end{tabular}

$\begin{array}{rrrr}\text { C } & -0.77119800 & 2.60572300 & -0.52325900 \\ \text { H } & -1.50104600 & 2.07478100 & -1.14562900 \\ \text { H } & -1.21226600 & 2.72657900 & 0.47216500 \\ \text { H } & -0.65580200 & 3.60420800 & -0.94857200 \\ \text { O } & -1.19908400 & -1.67113500 & 1.41273600 \\ \text { H } & -0.76368900 & -2.47171500 & 1.08192800 \\ \text { C } & -2.47724800 & 0.20667800 & 0.94260300 \\ \text { O } & -1.94164300 & 0.85877100 & 1.89242600 \\ \text { H } & -0.89552300 & 0.66448400 & 1.90650300 \\ \text { H } & 1.04089500 & 1.16521300 & 1.84792500\end{array}$

IM4b

Sum of electronic and thermal Free Energies $=-1193.881372$

\begin{tabular}{|c|c|c|c|}
\hline $\mathbf{C}$ & -2.93647300 & 0.14835500 & -0.37269200 \\
\hline $\mathbf{H}$ & -2.64839300 & 0.25982700 & -1.43046300 \\
\hline $\mathbf{C}$ & -2.11482400 & -2.62827600 & 0.08344300 \\
\hline $\mathbf{H}$ & -2.42758200 & -2.75484100 & 1.12705700 \\
\hline $\mathbf{H}$ & -1.66593300 & -3.58058700 & -0.21937500 \\
\hline $\mathbf{C}$ & -4.84740000 & -1.19161500 & 0.86592700 \\
\hline $\mathbf{H}$ & -5.26353900 & -0.25514600 & 1.24575000 \\
\hline $\mathbf{H}$ & -5.69269500 & -1.83850800 & 0.61371700 \\
\hline $\mathbf{H}$ & -4.31172800 & -1.68014900 & 1.68384500 \\
\hline $\mathbf{C}$ & -1.81027300 & -0.06305400 & 1.91188500 \\
\hline $\mathbf{H}$ & -0.95430200 & -0.47885500 & 2.45335000 \\
\hline $\mathbf{H}$ & -1.95946600 & 0.96403500 & 2.25820200 \\
\hline $\mathbf{H}$ & -2.68163600 & -0.64017600 & 2.21886200 \\
\hline $\mathbf{C}$ & -1.60962500 & -0.12863700 & 0.38458500 \\
\hline C & -0.58846400 & 0.98930100 & -0.05227100 \\
\hline $\mathbf{C}$ & -2.50461600 & 2.58021800 & 0.06310100 \\
\hline $\mathbf{H}$ & -2.87805100 & 3.60288200 & 0.08525700 \\
\hline C & -3.52662800 & 1.48489000 & 0.06975700 \\
\hline $\mathbf{H}$ & -4.36209500 & 1.75924400 & -0.58573300 \\
\hline $\mathbf{H}$ & -3.95382700 & 1.41147300 & 1.07878800 \\
\hline $\mathbf{C}$ & -1.17864800 & 2.39528300 & 0.03498300 \\
\hline $\mathbf{C}$ & 0.71150400 & 0.82847900 & 0.80022800 \\
\hline $\mathbf{H}$ & 0.72087700 & 1.53506600 & 1.63597100 \\
\hline $\mathbf{H}$ & 0.71045000 & -0.16804900 & 1.23985900 \\
\hline 0 & -0.17855600 & 0.76824000 & -1.40806500 \\
\hline $\mathbf{C}$ & 2.08522700 & 1.01056500 & 0.08356400 \\
\hline C & 2.15031700 & 0.52494900 & -1.35045700 \\
\hline C & 3.12989200 & -1.25278600 & 0.57454900 \\
\hline 0 & 2.51867000 & 1.16058800 & -2.27944700 \\
\hline C & 2.32511900 & -3.10862700 & -0.95966700 \\
\hline $\mathbf{H}$ & 2.28125100 & -3.75372500 & -0.08179300 \\
\hline $\mathbf{H}$ & 1.43940000 & -3.23800100 & -1.58350200 \\
\hline $\mathbf{H}$ & 3.20320500 & -3.39403700 & -1.55406600 \\
\hline $\mathbf{C}$ & 3.89177500 & -2.20705300 & 1.45882900 \\
\hline $\mathbf{H}$ & 4.50700700 & -2.88929200 & 0.86893400 \\
\hline $\mathbf{H}$ & 4.55132300 & -1.64875600 & 2.12385600 \\
\hline $\mathbf{H}$ & 3.21278700 & -2.79974200 & 2.07901800 \\
\hline $\mathbf{C}$ & -3.98613700 & -0.99338900 & -0.39022300 \\
\hline $\mathbf{H}$ & -4.68492500 & -0.71004000 & -1.18913600 \\
\hline $\mathbf{C}$ & -3.31899300 & -2.31182600 & -0.80332300 \\
\hline $\mathbf{H}$ & -2.97829200 & -2.24003900 & -1.84517700 \\
\hline $\mathbf{H}$ & -4.05066900 & -3.12585700 & -0.76413100 \\
\hline $\mathbf{C}$ & -0.23109400 & 3.56489600 & -0.04273700 \\
\hline $\mathbf{H}$ & 0.43208500 & 3.62799000 & 0.82481900 \\
\hline $\mathbf{H}$ & 0.41108800 & 3.49633000 & -0.92908200 \\
\hline $\mathbf{H}$ & -0.79060600 & 4.49962300 & -0.10910700 \\
\hline
\end{tabular}




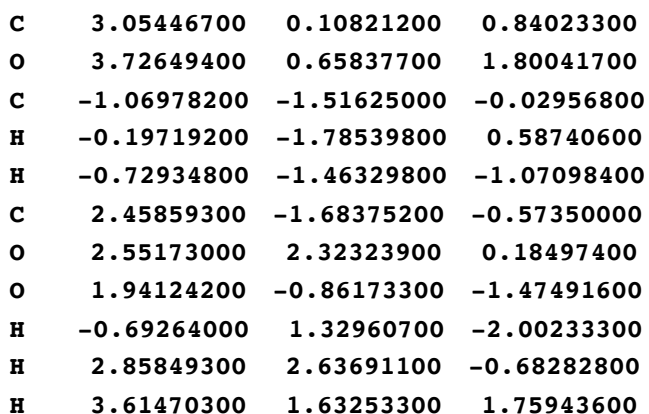

IM5

Sum of electronic and thermal Free Energies $=-1193.904132$

\begin{tabular}{|c|c|c|c|}
\hline C & 2.81571300 & 0.06515500 & 0.42573100 \\
\hline H & 2.41889700 & 0.12948700 & 1.45136800 \\
\hline C & 1.98736000 & -2.63786200 & -0.36398300 \\
\hline H & 2.39572500 & -2.66606100 & -1.38115900 \\
\hline H & 1.49167100 & -3.60166600 & -0.20512400 \\
\hline C & 4.83234200 & -1.22245600 & -0.69813500 \\
\hline H & 5.32222900 & -0.27326000 & -0.92796100 \\
\hline $\mathbf{H}$ & 5.61893200 & -1.92730100 & -0.41333600 \\
\hline H & 4.38701800 & -1.60607300 & -1.61986800 \\
\hline C & 1.92173800 & 0.04295600 & -1.97335600 \\
\hline $\mathbf{H}$ & 1.12885600 & -0.34368700 & -2.62150800 \\
\hline $\mathbf{H}$ & 2.09708100 & 1.08907700 & -2.24070800 \\
\hline H & 2.82581600 & -0.51145500 & -2.22015900 \\
\hline C & 1.56686500 & -0.11557100 & -0.47897200 \\
\hline C & 0.53757000 & 1.00971700 & -0.11982300 \\
\hline C & 2.50197400 & 2.52552900 & 0.12137100 \\
\hline H & 2.90236400 & 3.53276800 & 0.22255300 \\
\hline C & 3.48772100 & 1.40119600 & 0.12663900 \\
\hline H & 4.26762800 & 1.60286900 & 0.87087700 \\
\hline H & 4.00153600 & 1.37626200 & -0.84351000 \\
\hline C & 1.17562700 & 2.38743600 & 0.01605600 \\
\hline C & -0.68743700 & 0.97868100 & -1.08108700 \\
\hline H & -0.70197200 & 1.82187800 & -1.77442800 \\
\hline H & -0.68431500 & 0.05993000 & -1.66896000 \\
\hline 0 & -0.07111100 & 0.74147300 & 1.20475600 \\
\hline C & -1.94599300 & 1.02282000 & -0.19392300 \\
\hline C & -1.40472900 & 0.54008300 & 1.16661700 \\
\hline C & -3.15215900 & -1.20204000 & -0.45016000 \\
\hline 0 & -2.07428200 & 1.14810000 & 2.18745700 \\
\hline C & -2.38793500 & -3.08821500 & 1.05099200 \\
\hline H & -1.91374900 & -3.68961300 & 0.26579900 \\
\hline H & -1.84288200 & -3.21434700 & 1.98487200 \\
\hline H & -3.41558800 & -3.44651800 & 1.15637400 \\
\hline C & -4.12014500 & -2.11125300 & -1.16270200 \\
\hline H & -4.95535500 & -2.39246300 & -0.51484800 \\
\hline H & -4.53051000 & -1.60761300 & -2.03772200 \\
\hline H & -3.62550300 & -3.02554100 & -1.49995700 \\
\hline C & 3.82952800 & -1.11077600 & 0.46061100 \\
\hline H & 4.43378400 & -0.92148700 & 1.35813400 \\
\hline C & 3.10135100 & -2.44604100 & 0.66536700 \\
\hline H & 2.65591400 & -2.47235100 & 1.66922000 \\
\hline H & 3.82063500 & -3.27040800 & 0.61630400 \\
\hline C & 0.27027900 & 3.58532700 & 0.13944200 \\
\hline H & -0.37324400 & 3.73418200 & -0.73185500 \\
\hline $\mathbf{H}$ & -0.38870500 & 3.48761100 & 1.00954800 \\
\hline
\end{tabular}

$\begin{array}{rrrr}\text { H } & 0.86781200 & 4.48812500 & 0.27611900 \\ \text { C } & -3.03174800 & 0.13413100 & -0.75675800 \\ \text { O } & -3.82337100 & 0.69518900 & -1.62953100 \\ \text { C } & 0.96401500 & -1.51053700 & -0.21890800 \\ \text { H } & 0.10997200 & -1.69217400 & -0.89087900 \\ \text { H } & 0.58903000 & -1.54298800 & 0.80935500 \\ \text { C } & -2.37678700 & -1.66183600 & 0.63038600 \\ \text { O } & -2.45351200 & 2.32906800 & -0.12867300 \\ \text { O } & -1.60790700 & -0.91466900 & 1.36230400 \\ \text { H } & -1.49905600 & 1.20631200 & 2.96613300 \\ \text { H } & -2.79777300 & 2.50242200 & 0.76313900 \\ \text { H } & -3.67569300 & 1.66214700 & -1.63074900\end{array}$

IM6a

Sum of electronic and thermal Free Energies $=-1193.885198$

\begin{tabular}{|c|c|c|c|}
\hline C & 2.85757300 & -0.02453800 & 0.38102200 \\
\hline H & 2.53226300 & 0.14419600 & 1.41902300 \\
\hline C & 1.69982600 & -2.66935700 & -0.15584600 \\
\hline $\mathbf{H}$ & 2.04213500 & -2.82285600 & -1.18618500 \\
\hline H & 1.11632900 & -3.55727100 & 0.11018400 \\
\hline C & 4.64517400 & -1.59993600 & -0.76194000 \\
\hline H & 5.21015200 & -0.72704400 & -1.09812400 \\
\hline H & 5.37590400 & -2.35986000 & -0.47054200 \\
\hline $\mathbf{H}$ & 4.10094100 & -2.00240000 & -1.62037100 \\
\hline C & 1.82512100 & -0.10127600 & -1.96229400 \\
\hline H & 0.95271100 & -0.42047500 & -2.54110900 \\
\hline H & 2.11072400 & 0.89698500 & -2.30608400 \\
\hline H & 2.63759200 & -0.77892500 & -2.21993600 \\
\hline C & 1.54763600 & -0.13054200 & -0.44475100 \\
\hline C & 0.66563900 & 1.11533700 & -0.10274000 \\
\hline C & 2.77658500 & 2.43430800 & -0.07894400 \\
\hline H & 3.28182800 & 3.39856700 & -0.07207200 \\
\hline C & 3.64167400 & 1.21454000 & -0.04291100 \\
\hline H & 4.47567700 & 1.38413900 & 0.64846300 \\
\hline H & 4.09857500 & 1.07820500 & -1.03208500 \\
\hline C & 1.43926500 & 2.42493800 & -0.10200600 \\
\hline C & -0.64055800 & 1.13945700 & -0.95550300 \\
\hline H & -0.65780600 & 1.95509600 & -1.68191700 \\
\hline H & -0.74042100 & 0.20905800 & -1.51342100 \\
\hline 0 & 0.16604900 & 0.95988200 & 1.28327900 \\
\hline C & -1.79874600 & 1.26564400 & 0.04698700 \\
\hline C & -1.13930300 & 0.89900200 & 1.38419300 \\
\hline C & -3.27048800 & -0.92253500 & -0.39324900 \\
\hline 0 & -1.74136200 & 0.63270500 & 2.40325400 \\
\hline C & -2.52580400 & -3.33791800 & -0.12114900 \\
\hline H & -2.51961900 & -3.40380900 & -1.20847700 \\
\hline H & -1.63279700 & -3.79939900 & 0.30397000 \\
\hline H & -3.39297900 & -3.89122200 & 0.26069900 \\
\hline C & -4.37945700 & -1.37494000 & -1.33332500 \\
\hline н & -5.29833100 & -0.83613000 & -1.09276600 \\
\hline H & -4.13341100 & -1.15408100 & -2.37569500 \\
\hline H & -4.58542600 & -2.43870000 & -1.23602800 \\
\hline C & 3.74232500 & -1.29768100 & 0.44351400 \\
\hline н & 4.42180000 & -1.11494000 & 1.28671100 \\
\hline C & 2.88711400 & -2.52215500 & 0.79651900 \\
\hline н & 2.50313800 & -2.42046000 & 1.82028000 \\
\hline H & 3.50792500 & -3.42395000 & 0.77859500 \\
\hline C & 0.66497600 & 3.71547400 & -0.00626600 \\
\hline н & 0.00089600 & 3.88574200 & -0.86414600 \\
\hline
\end{tabular}




$\begin{array}{lrrr}\text { H } & 0.08682900 & 3.75940800 & 0.92824200 \\ \text { H } & 1.34757200 & 4.56615500 & 0.02394400 \\ \text { C } & -3.02456700 & 0.44325300 & -0.38345800 \\ \text { O } & -3.88045200 & 1.16518100 & -1.06840300 \\ \text { C } & 0.80743700 & -1.42981700 & -0.06326800 \\ \text { H } & -0.07744500 & -1.56424500 & -0.70596800 \\ \text { H } & 0.45563300 & -1.36026900 & 0.97298700 \\ \text { C } & -2.60199800 & -1.92736000 & 0.35670700 \\ \text { O } & -2.30723700 & 2.58025200 & 0.17673700 \\ \text { O } & -2.07339100 & -1.78043200 & 1.52432000 \\ \text { H } & -1.59625200 & 3.23407100 & 0.12240500 \\ \text { H } & -3.65622500 & 2.11372500 & -0.97088000 \\ \text { H } & -2.19332700 & -0.89340100 & 1.95905000\end{array}$

IM6b

Sum of electronic and thermal Free Energies $=-1193.899371$

\begin{tabular}{|c|c|c|c|}
\hline C & 3.16580800 & 0.26571000 & 0.56601500 \\
\hline H & 2.66627500 & 0.42949600 & 1.53346500 \\
\hline C & 2.76444000 & -2.62110600 & 0.21867000 \\
\hline $\mathbf{H}$ & 3.28087800 & -2.77623500 & -0.73627700 \\
\hline $\mathbf{H}$ & 2.37606900 & -3.59951100 & 0.51859000 \\
\hline C & 5.41791100 & -0.89575200 & -0.17358900 \\
\hline $\mathbf{H}$ & 5.79909000 & 0.06014100 & -0.54183600 \\
\hline $\mathbf{H}$ & 6.26152300 & -1.42185400 & 0.28256900 \\
\hline H & 5.10676300 & -1.49083600 & -1.03592200 \\
\hline C & 2.51838100 & -0.26120000 & -1.85876800 \\
\hline $\mathbf{H}$ & 1.81085500 & -0.79924900 & -2.49683200 \\
\hline $\mathbf{H}$ & 2.64001500 & 0.74677200 & -2.26579300 \\
\hline $\mathbf{H}$ & 3.47704800 & -0.76942100 & -1.95137300 \\
\hline C & 2.05064700 & -0.23975400 & -0.38881600 \\
\hline C & 0.86219200 & 0.76231400 & -0.30109900 \\
\hline C & 2.55838300 & 2.58060600 & -0.16409000 \\
\hline H & 2.80552400 & 3.64035000 & -0.20081300 \\
\hline C & 3.67959300 & 1.62673800 & 0.10262200 \\
\hline $\mathbf{H}$ & 4.34401300 & 2.05448100 & 0.86297100 \\
\hline $\mathbf{H}$ & 4.28837500 & 1.53544600 & -0.80678800 \\
\hline C & 1.28216000 & 2.22355700 & -0.34485200 \\
\hline $\mathbf{C}$ & -0.29917300 & 0.41667500 & -1.27656300 \\
\hline H & -0.38402600 & 1.14245100 & -2.08673300 \\
\hline H & -0.12895800 & -0.55593000 & -1.72886400 \\
\hline 0 & 0.22842000 & 0.61442700 & 1.04815500 \\
\hline C & -1.59372700 & 0.40375200 & -0.44860300 \\
\hline C & -1.05962400 & 0.54009600 & 0.99775100 \\
\hline C & -3.72250000 & -1.05822000 & -0.20029900 \\
\hline 0 & -1.78590500 & 0.59808900 & 1.98588900 \\
\hline C & -4.38764500 & -2.37084800 & -0.57448200 \\
\hline H & -5.45478200 & -2.36179000 & -0.35848100 \\
\hline H & -4.27434800 & -2.59393100 & -1.64101400 \\
\hline H & -3.97297400 & -3.20172200 & 0.00978500 \\
\hline c & 4.31269400 & -0.72963700 & 0.87981700 \\
\hline H & 4.80576500 & -0.30731900 & 1.76595700 \\
\hline 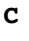 & 3.73293200 & -2.09398800 & 1.27705500 \\
\hline H & 3.19352000 & -1.99977200 & 2.22921400 \\
\hline $\mathbf{H}$ & 4.54755500 & -2.80677900 & 1.44363300 \\
\hline C & 0.20057400 & 3.26502200 & -0.48743000 \\
\hline H & -0.31797100 & 3.21544200 & -1.45309700 \\
\hline $\mathbf{H}$ & -0.53694200 & 3.18630500 & 0.32593500 \\
\hline $\mathbf{H}$ & 0.62574700 & 4.26695500 & -0.41092800 \\
\hline C & -2.40590900 & -0.89303500 & -0.52145400 \\
\hline
\end{tabular}

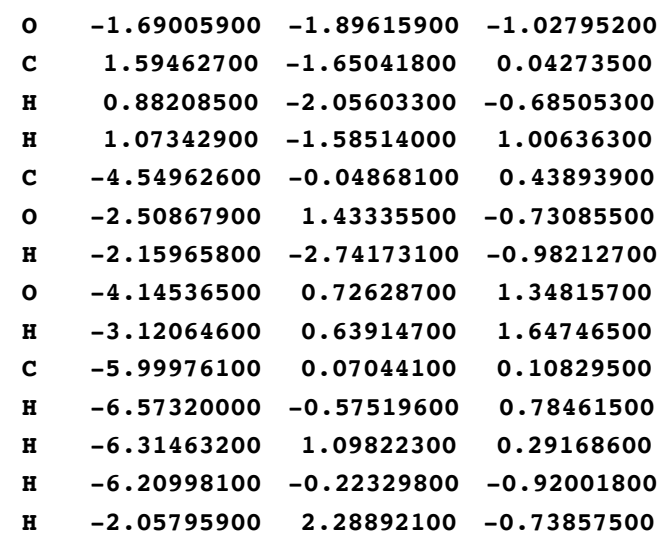

IM7

Sum of electronic and thermal Free Energies $=-1193.908568$

\begin{tabular}{|c|c|c|c|}
\hline C & 3.12855600 & 0.41135600 & 0.49263000 \\
\hline & 2.66746300 & 0.45387500 & 1.49191200 \\
\hline & 3.05792600 & -2.48205200 & 0.00586100 \\
\hline & 3.53380900 & -2.53207000 & -0.98077000 \\
\hline & 2.80439000 & -3.51222300 & 0.27423600 \\
\hline & 5.45528000 & -0.42259100 & -0.43097800 \\
\hline & 5.69064400 & 0.58969700 & -0.76946300 \\
\hline & 6.38420700 & -0.85622500 & -0.04966100 \\
\hline & 5.16928500 & -1.01363000 & -1.30460100 \\
\hline & 2.41758700 & -0.07265900 & -1.92810500 \\
\hline & 1.71958800 & -0.63022400 & -2.55954800 \\
\hline & 2.43383900 & 0.96406800 & -2.27589800 \\
\hline & 3.40701700 & -0.49310900 & -2.10028300 \\
\hline & 2.03461200 & -0.18040200 & -0.43661700 \\
\hline & 0.75053300 & 0.66754100 & -0.25598100 \\
\hline & 2.19867900 & 2.66871600 & -0.06234100 \\
\hline H & 2.31017500 & 3.75173500 & -0.05034200 \\
\hline C & 3.44163300 & 1.85189000 & 0.09588500 \\
\hline $\mathbf{H}$ & 4.08535900 & 2.31640700 & 0.85240200 \\
\hline $\mathbf{H}$ & 4.01018800 & 1.89388600 & -0.84285400 \\
\hline C & 0.96705800 & 2.16590900 & -0.21357100 \\
\hline C & -0.43165500 & 0.23487800 & -1.16009100 \\
\hline H & -0.64376000 & 0.97442700 & -1.93237300 \\
\hline H & -0.20904200 & -0.71571300 & -1.64427400 \\
\hline 0 & 0.19455400 & 0.37660200 & 1.16151800 \\
\hline C & -1.65327300 & 0.04140400 & -0.26113800 \\
\hline C & -1.05551900 & 0.21864900 & 1.13533200 \\
\hline C & -3.67682700 & -1.14249600 & -0.31583700 \\
\hline 0 & -1.73869500 & 0.23456100 & 2.20653500 \\
\hline C & -4.77369600 & -2.15479300 & -0.32970000 \\
\hline $\mathbf{n}$ & -5.42762800 & -2.01472600 & -1.19591600 \\
\hline H & -4.38678400 & -3.17604800 & -0.37085700 \\
\hline H & -5.38557700 & -2.06565000 & 0.57350800 \\
\hline C & 4.40405100 & -0.44742100 & 0.68830800 \\
\hline $\mathbf{H}$ & 4.89417000 & -0.01103200 & 1.56926400 \\
\hline $\mathbf{C}$ & 4.01673500 & -1.89063700 & 1.03883300 \\
\hline $\mathbf{H}$ & 3.52789600 & -1.90997300 & 2.02230000 \\
\hline $\mathbf{H}$ & 4.91956700 & -2.50480600 & 1.12245500 \\
\hline $\mathbf{C}$ & -0.25049700 & 3.05338500 & -0.26895500 \\
\hline & -0.79969200 & 2.97403100 & -1.21183800 \\
\hline & -0.96340000 & 2.81852100 & 0.53259800 \\
\hline & 0.04252700 & 4.09632300 & -0.13938400 \\
\hline
\end{tabular}




$\begin{array}{lrrr}\text { C } & -2.35377300 & -1.29868300 & -0.36363000 \\ \text { O } & -1.55356200 & -2.37573800 & -0.44119900 \\ \text { C } & 1.77185600 & -1.65525300 & -0.06144500 \\ \text { H } & 1.07234800 & -2.11212400 & -0.77031900 \\ \text { H } & 1.29845500 & -1.70213500 & 0.92754600 \\ \text { C } & -3.93363900 & 0.32941500 & -0.09880100 \\ \text { O } & -2.70176700 & 0.97335200 & -0.42068800 \\ \text { O } & -4.14936800 & 0.47171300 & 1.32182600 \\ \text { H } & -4.56137200 & 1.32705400 & 1.51959700 \\ \text { C } & -5.05289200 & 0.97112300 & -0.87941200 \\ \text { H } & -6.01176800 & 0.53856600 & -0.58517200 \\ \text { H } & -5.06957500 & 2.04942300 & -0.69484900 \\ \text { H } & -4.89513300 & 0.80736900 & -1.94661000 \\ \text { H } & -2.74183800 & 0.24539400 & 1.99629400 \\ \text { H } & -2.06105600 & -3.19866700 & -0.42251700\end{array}$

IM8 Sum of electronic and thermal Free Energies $=-1193.918164$

\begin{tabular}{|c|c|c|c|}
\hline C & 3.16614000 & 0.41419100 & 0.52518200 \\
\hline & 2.69810600 & 0.43684100 & 1.52144600 \\
\hline & 3.13781600 & -2.46951700 & -0.02431700 \\
\hline H & 3.62067100 & -2.48596500 & -1.00891200 \\
\hline & 2.89906200 & -3.51004300 & 0.21688100 \\
\hline & 5.51074700 & -0.37325200 & -0.39702100 \\
\hline 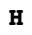 & 5.74111400 & 0.64909200 & -0.70738300 \\
\hline 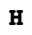 & 6.43984100 & -0.81010500 & -0.01943500 \\
\hline $\mathbf{H}$ & 5.23587500 & -0.94371600 & -1.28797200 \\
\hline : & 2.46911500 & -0.02388000 & -1.90385400 \\
\hline H & 1.80272200 & -0.60087100 & -2.55313100 \\
\hline I & 2.44218800 & 1.01897800 & -2.23288200 \\
\hline $\mathbf{H}$ & 3.47716600 & -0.39667900 & -2.07927000 \\
\hline C & 2.08084700 & -0.17170100 & -0.41854100 \\
\hline C & 0.77725800 & 0.65292800 & -0.19535500 \\
\hline C & 2.21578200 & 2.67151100 & 0.00025000 \\
\hline H & 2.31714900 & 3.75507800 & 0.03292000 \\
\hline C & 3.46699500 & 1.86330800 & 0.14919800 \\
\hline H & 4.10417200 & 2.32123100 & 0.91533200 \\
\hline H & 4.03910400 & 1.92153600 & -0.78641600 \\
\hline 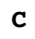 & 0.99090700 & 2.15827000 & -0.15457900 \\
\hline C & -0.36111500 & 0.22580700 & -1.17070300 \\
\hline $\mathbf{H}$ & -0.54207400 & 0.96256800 & -1.95419500 \\
\hline $\mathbf{H}$ & -0.11168700 & -0.72357500 & -1.64627600 \\
\hline 0 & 0.23254300 & 0.31105200 & 1.13671500 \\
\hline C & -1.59492200 & 0.00896900 & -0.31009500 \\
\hline C & -1.05311200 & 0.06317400 & 1.15519100 \\
\hline C & -3.71282200 & -0.95818800 & -0.65545700 \\
\hline 0 & -1.73573100 & -0.10893100 & 2.12923300 \\
\hline C & -4.88635000 & -1.87631200 & -0.76656800 \\
\hline H & -5.53641800 & -1.59216800 & -1.59725300 \\
\hline H & -4.57431500 & -2.90948800 & -0.93857200 \\
\hline $\mathbf{H}$ & -5.45943700 & -1.84046800 & 0.16466000 \\
\hline C & 4.45062600 & -0.43351500 & 0.71267700 \\
\hline $\mathbf{H}$ & 4.92909400 & -0.01009300 & 1.60627800 \\
\hline C & 4.08029600 & -1.88877300 & 1.02953700 \\
\hline H & 3.58301900 & -1.93577700 & 2.00765100 \\
\hline $\mathbf{H}$ & 4.99047200 & -2.49301300 & 1.10725000 \\
\hline C & -0.23422500 & 3.03579300 & -0.18353200 \\
\hline H & -0.79281000 & 2.96443500 & -1.12278300 \\
\hline & -0.92799900 & 2.78320900 & 0.62861500 \\
\hline
\end{tabular}

$\begin{array}{rrrr}\text { H } & 0.05012200 & 4.08056200 & -0.04862900 \\ \text { C } & -2.38059700 & -1.24230500 & -0.51773800 \\ \text { O } & -1.72815200 & -2.37858500 & -0.49076200 \\ \text { C } & 1.84112000 & -1.65837600 & -0.07595800 \\ \text { H } & 1.15422200 & -2.11326100 & -0.80125800 \\ \text { H } & 1.36481500 & -1.73195900 & 0.90916500 \\ \text { C } & -3.77471800 & 0.45020400 & -0.57347300 \\ \text { O } & -2.60518900 & 1.02435100 & -0.42649500 \\ \text { O } & -4.59237400 & 0.02109700 & 1.94772100 \\ \text { H } & -5.17218400 & 0.25069500 & 2.68222100 \\ \text { C } & -4.95480800 & 1.32123300 & -0.70600700 \\ \text { H } & -5.83381500 & 0.81035800 & -0.31300600 \\ \text { H } & -4.79171200 & 2.25812100 & -0.17344400 \\ \text { H } & -5.11065300 & 1.53681500 & -1.77061300 \\ \text { H } & -3.70143500 & -0.07170300 & 2.32011300 \\ \text { H } & -2.31301600 & -3.14990200 & -0.55541600\end{array}$

TS_1-2a

Sum of electronic and thermal Free Energies $=-1193.842165$

\begin{tabular}{|c|c|c|c|}
\hline C & -2.80213700 & -0.81195000 & 0.22684700 \\
\hline $\mathbf{H}$ & -2.20674000 & -1.28569000 & 1.02261500 \\
\hline C & -1.76270800 & 1.43712000 & 0.75353400 \\
\hline $\mathbf{H}$ & -1.16009300 & 1.05378800 & 1.58496700 \\
\hline $\mathbf{H}$ & -1.21783900 & 2.29565300 & 0.33795800 \\
\hline C & -3.10964900 & 1.89817400 & 1.31296900 \\
\hline $\mathbf{H}$ & -3.71877800 & 2.35523000 & 0.52363000 \\
\hline $\mathbf{H}$ & -2.94027200 & 2.67984800 & 2.06022600 \\
\hline $\mathbf{C}$ & -5.31209300 & -0.01816800 & 0.02554100 \\
\hline $\mathbf{H}$ & -5.48522300 & -0.74542700 & -0.77178200 \\
\hline $\mathbf{H}$ & -6.21855800 & 0.00817400 & 0.63730800 \\
\hline $\mathbf{H}$ & -5.21449000 & 0.96943300 & -0.43232600 \\
\hline $\mathbf{C}$ & -2.47856700 & 0.93429300 & -1.62114900 \\
\hline $\mathbf{H}$ & -1.97236900 & 1.86717200 & -1.88826200 \\
\hline $\mathbf{H}$ & -2.38332500 & 0.24513100 & -2.46520000 \\
\hline $\mathbf{H}$ & -3.53573600 & 1.17108800 & -1.50992500 \\
\hline C & -1.90962100 & 0.33642300 & -0.31739600 \\
\hline C & -0.52258000 & -0.27646500 & -0.65306100 \\
\hline C & -1.72451800 & -2.28472000 & -1.49140500 \\
\hline $\mathbf{H}$ & -1.71886200 & -3.22573300 & -2.03937800 \\
\hline C & -3.01469100 & -1.87210300 & -0.85230900 \\
\hline $\mathbf{H}$ & -3.50279000 & -2.75308200 & -0.41826600 \\
\hline $\mathbf{H}$ & -3.69555400 & -1.51073800 & -1.63461600 \\
\hline C & -0.58741900 & -1.58397000 & -1.42687000 \\
\hline C & 0.46251600 & 0.75190200 & -1.30966200 \\
\hline $\mathbf{H}$ & 0.69924100 & 0.48711300 & -2.34099300 \\
\hline $\mathbf{H}$ & 0.05222100 & 1.76302800 & -1.30500800 \\
\hline 0 & 0.15611000 & -0.67010000 & 0.63585000 \\
\hline C & 1.67295500 & 0.68696100 & -0.41129300 \\
\hline C & 1.39991100 & -0.24635500 & 0.64988600 \\
\hline C & 3.10235200 & 0.86405400 & -0.79911500 \\
\hline C & 2.47195200 & -1.02599700 & 1.27824400 \\
\hline C & 3.68910800 & -0.91790300 & 0.71874400 \\
\hline 0 & 3.97693800 & 0.00927900 & -0.31265000 \\
\hline 0 & 3.48174800 & 1.84660600 & -1.42995800 \\
\hline C & 4.94599500 & -1.64520100 & 1.04284400 \\
\hline $\mathbf{H}$ & 5.70228400 & -0.94147100 & 1.40236500 \\
\hline $\mathbf{H}$ & 4.78152900 & -2.40856200 & 1.79957900 \\
\hline $\mathbf{H}$ & 5.33589100 & -2.11993500 & 0.13803900 \\
\hline C & 2.09192900 & -1.94525100 & 2.40236600 \\
\hline
\end{tabular}




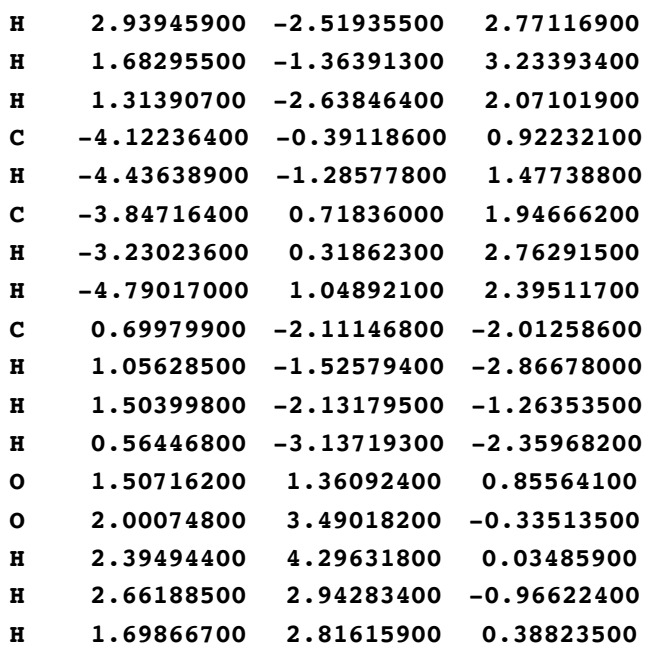

TS_2b-3

Sum of electronic and thermal Free Energies $=-1193.843356$

\begin{tabular}{|c|c|c|c|}
\hline C & 2.66113700 & 0.76222800 & 0.41040600 \\
\hline H & 2.09887700 & 0.84363000 & 1.35503000 \\
\hline C & 1.84364800 & -1.61045500 & 0.03358800 \\
\hline $\mathbf{H}$ & 1.30527200 & -1.68121600 & 0.99071000 \\
\hline H & 1.32805300 & -2.28391700 & -0.65779900 \\
\hline C & 3.26189300 & -2.11261000 & 0.31208200 \\
\hline H & 3.84210200 & -2.15033000 & -0.61692600 \\
\hline H & 3.20959300 & -3.14144300 & 0.68075600 \\
\hline C & 5.20662200 & 0.36388700 & -0.17117000 \\
\hline $\mathbf{H}$ & 5.29127200 & 1.37067900 & -0.58682900 \\
\hline H & 6.14810900 & 0.15212000 & 0.34361600 \\
\hline $\mathbf{H}$ & 5.13838700 & -0.34147400 & -1.00285800 \\
\hline C & 2.32266700 & -0.16376900 & -1.96000700 \\
\hline H & 1.85806800 & -0.96078300 & -2.54780500 \\
\hline H & 2.13109700 & 0.78596700 & -2.46480000 \\
\hline $\mathbf{H}$ & 3.39687400 & -0.33959100 & -1.98695200 \\
\hline C & 1.81087000 & -0.16533200 & -0.50309600 \\
\hline C & 0.35669200 & 0.40845400 & -0.52865000 \\
\hline C & 1.39301400 & 2.66303200 & -0.63457900 \\
\hline $\mathbf{H}$ & 1.31269300 & 3.72828300 & -0.84450500 \\
\hline C & 2.72824300 & 2.16765800 & -0.17988000 \\
\hline $\mathbf{H}$ & 3.15155900 & 2.86366400 & 0.55414000 \\
\hline $\mathbf{H}$ & 3.40891900 & 2.19652600 & -1.04145800 \\
\hline C & 0.31067200 & 1.90177000 & -0.83354800 \\
\hline C & -0.64745100 & -0.43622100 & -1.36146400 \\
\hline H & -1.24891500 & 0.20874900 & -2.00450700 \\
\hline H & -0.15002300 & -1.16414300 & -2.00278800 \\
\hline 0 & -0.23752300 & 0.30361900 & 0.86479600 \\
\hline C & -1.56778300 & -1.21545800 & -0.39048000 \\
\hline C & -2.97540500 & -1.34805300 & -0.96045800 \\
\hline C & -2.65224600 & 0.51764700 & 1.17037900 \\
\hline C & -3.69755800 & 0.53194100 & 0.32568500 \\
\hline 0 & -3.83610900 & -0.33866100 & -0.74901200 \\
\hline 0 & -3.27346500 & -2.30386500 & -1.61670800 \\
\hline C & -4.87560900 & 1.44249200 & 0.34889800 \\
\hline H & -4.95363600 & 1.94389600 & -0.62063800 \\
\hline H & -5.78923000 & 0.85752000 & 0.48867100 \\
\hline H & -4.80076600 & 2.19367800 & 1.13114400 \\
\hline C & -2.47885000 & 1.45292700 & 2.33851600 \\
\hline
\end{tabular}

$\begin{array}{lrrr}\text { H } & -2.03294200 & 0.92142000 & 3.18339900 \\ \text { H } & -1.81406800 & 2.28273200 & 2.07612600 \\ \text { H } & -3.42997400 & 1.85910800 & 2.67767400 \\ \text { C } & 4.05629800 & 0.23163300 & 0.83766500 \\ \text { H } & 4.33706300 & 0.86156500 & 1.69263500 \\ \text { C } & 3.94368600 & -1.21315400 & 1.34142500 \\ \text { H } & 3.35689400 & -1.23500900 & 2.27101600 \\ \text { H } & 4.93828800 & -1.59648900 & 1.59095400 \\ \text { C } & -0.98448500 & 2.52024500 & -1.30127200 \\ \text { H } & -1.22099400 & 2.26377300 & -2.33987400 \\ \text { H } & -1.83539600 & 2.21428900 & -0.68216200 \\ \text { H } & -0.91443600 & 3.60778900 & -1.24697300 \\ \text { O } & -1.09991200 & -2.50631200 & -0.09090200 \\ \text { H } & -1.56554300 & -3.13971600 & -0.66596900 \\ \text { C } & -1.51188100 & -0.38184400 & 0.89429400 \\ \text { O } & -1.09915500 & -1.19226900 & 2.04673500 \\ \text { H } & -1.06898700 & -2.13395000 & 1.75232100 \\ \text { H } & -0.08395300 & -0.58952300 & 1.75536800\end{array}$

TS_3-4a

Sum of electronic and thermal Free Energies $=-1193.875480$

\begin{tabular}{|c|c|c|c|}
\hline C & 2.78762800 & 0.76776600 & 0.40749500 \\
\hline H & 2.31663300 & 0.94038000 & 1.39345100 \\
\hline C & 1.77247400 & -1.54776700 & 0.18344700 \\
\hline H & 1.28046100 & -1.54447400 & 1.16978000 \\
\hline H & 1.17736100 & -2.19658400 & -0.46344900 \\
\hline C & 3.16650400 & -2.14258300 & 0.38962600 \\
\hline H & 3.67645100 & -2.24877500 & -0.57521700 \\
\hline H & 3.06926800 & -3.15361000 & 0.79672700 \\
\hline C & 5.24932600 & 0.15226400 & -0.32353500 \\
\hline H & 5.35470700 & 1.12445800 & -0.81128200 \\
\hline H & 6.21180300 & -0.07729300 & 0.14241700 \\
\hline H & 5.08834000 & -0.60168600 & -1.09817400 \\
\hline C & 2.22892100 & -0.19355300 & -1.88808200 \\
\hline H & 1.78658200 & -1.05980400 & -2.38849500 \\
\hline H & 1.94234400 & 0.70654700 & -2.44032900 \\
\hline H & 3.30915100 & -0.29893300 & -1.97713200 \\
\hline C & 1.80349600 & -0.12203900 & -0.40573900 \\
\hline C & 0.38284200 & 0.54818400 & -0.33183800 \\
\hline C & 1.57341500 & 2.74296900 & -0.52379900 \\
\hline H & 1.53798500 & 3.81999500 & -0.67796700 \\
\hline C & 2.91399900 & 2.14089400 & -0.24479400 \\
\hline H & 3.49082100 & 2.81185200 & 0.40273700 \\
\hline H & 3.47886500 & 2.07435000 & -1.18382200 \\
\hline C & 0.42476100 & 2.06045800 & -0.58168800 \\
\hline C & -0.67700600 & -0.13999000 & -1.24007400 \\
\hline H & -1.32941700 & 0.62190000 & -1.66896500 \\
\hline H & -0.22026100 & -0.66840000 & -2.07786900 \\
\hline 0 & -0.21029500 & 0.40365300 & 0.99140400 \\
\hline C & -1.57612100 & -1.18250100 & -0.51181600 \\
\hline C & -2.92218200 & -1.14667900 & -1.23592600 \\
\hline C & -2.79248600 & 0.21074600 & 1.24116700 \\
\hline C & -3.64985300 & 0.55995400 & 0.25138200 \\
\hline 0 & -3.71130400 & -0.07530700 & -0.96998600 \\
\hline 0 & -3.22264800 & -1.96368800 & -2.05042700 \\
\hline C & -4.70197500 & 1.60704300 & 0.32302500 \\
\hline H & -4.62101900 & 2.24645000 & -0.56132300 \\
\hline H & -5.69201600 & 1.14066000 & 0.29825900 \\
\hline H & -4.60510600 & 2.21690900 & 1.21871600 \\
\hline
\end{tabular}




$\begin{array}{lrrr}\text { C } & -2.80467800 & 0.81679100 & 2.62020400 \\ \text { H } & -2.37634300 & 0.11416900 & 3.33752100 \\ \text { H } & -2.20439500 & 1.73213400 & 2.64046200 \\ \text { H } & -3.81644100 & 1.04891800 & 2.95222500 \\ \text { C } & 4.16538000 & 0.14722800 & 0.76499600 \\ \text { H } & 4.55096800 & 0.78719400 & 1.57008800 \\ \text { C } & 3.98138800 & -1.26427700 & 1.33618100 \\ \text { H } & 3.45906200 & -1.20764300 & 2.30217400 \\ \text { H } & 4.96020500 & -1.71220500 & 1.53612600 \\ \text { C } & -0.88821200 & 2.78248900 & -0.76596900 \\ \text { H } & -1.31805300 & 2.64766600 & -1.76486800 \\ \text { H } & -1.62786100 & 2.44048000 & -0.03085700 \\ \text { H } & -0.74875900 & 3.85459500 & -0.61849500 \\ \text { O } & -1.06815200 & -2.48399600 & -0.53937800 \\ \text { H } & -1.56629800 & -3.00465300 & -1.19380400 \\ \text { C } & -1.81635500 & -0.80680500 & 0.93954700 \\ \text { O } & -1.50420100 & -1.67714700 & 1.84895400 \\ \text { H } & -1.02760000 & -2.42383500 & 1.42621100 \\ \text { H } & 0.46775900 & 0.29755500 & 1.67776100\end{array}$

TS_4b-5

Sum of electronic and thermal Free Energies $=-1193.840211$

\begin{tabular}{|c|c|c|c|}
\hline C & -2.81358900 & 0.01754300 & -0.42111900 \\
\hline $\mathbf{H}$ & -2.40580900 & 0.06729700 & -1.44366300 \\
\hline C & -1.94226000 & -2.66578700 & 0.38544300 \\
\hline $\mathbf{H}$ & -2.35889600 & -2.70496800 & 1.39880900 \\
\hline $\mathbf{H}$ & -1.42671300 & -3.61910200 & 0.22913900 \\
\hline C & -4.80997800 & -1.29607000 & 0.70516300 \\
\hline H & -5.30856700 & -0.35180600 & 0.93726300 \\
\hline $\mathbf{H}$ & -5.59026000 & -2.00695200 & 0.41865100 \\
\hline $\mathbf{H}$ & -4.36178400 & -1.67808900 & 1.62603700 \\
\hline C & -1.94433000 & 0.03355200 & 1.99071200 \\
\hline H & -1.13726000 & -0.30289400 & 2.64878900 \\
\hline H & -2.17043900 & 1.07447600 & 2.23916200 \\
\hline $\mathbf{H}$ & -2.82214200 & -0.55968100 & 2.24149600 \\
\hline C & -1.57541200 & -0.13545900 & 0.50181500 \\
\hline C & -0.56859700 & 1.00374100 & 0.14342900 \\
\hline C & -2.53389100 & 2.49011100 & -0.14737400 \\
\hline $\mathbf{H}$ & -2.94711000 & 3.49122500 & -0.25716200 \\
\hline C & -3.50387100 & 1.35179600 & -0.15578400 \\
\hline H & -4.27177700 & 1.53431700 & -0.91697100 \\
\hline H & -4.03557700 & 1.33895800 & 0.80499900 \\
\hline C & -1.20638800 & 2.37393800 & -0.01912600 \\
\hline C & 0.67952000 & 0.97751700 & 1.07598600 \\
\hline H & 0.69018600 & 1.82036200 & 1.77016200 \\
\hline H & 0.67550700 & 0.06137800 & 1.66754400 \\
\hline 0 & 0.03431100 & 0.67634700 & -1.19403300 \\
\hline C & 1.95706700 & 1.02668600 & 0.20418700 \\
\hline c & 1.50402700 & 0.55895300 & -1.20003000 \\
\hline C & 3.16130500 & -1.19083000 & 0.45466000 \\
\hline 0 & 1.76478100 & 1.34740200 & -2.19876300 \\
\hline C & 2.57610700 & -3.00503600 & -1.20467700 \\
\hline H & 2.06620400 & -3.67088100 & -0.49784600 \\
\hline H & 2.11125900 & -3.08704000 & -2.18559600 \\
\hline H & 3.62135100 & -3.32298500 & -1.25088800 \\
\hline C & 4.10424300 & -2.11217800 & 1.18546600 \\
\hline H & 5.00113700 & -2.31763500 & 0.59389600 \\
\hline H & 4.42009300 & -1.65540400 & 2.12333300 \\
\hline $\mathbf{H}$ & 3.62111900 & -3.06406500 & 1.41824000 \\
\hline
\end{tabular}

$\begin{array}{lrrr}\text { C } & -3.80810700 & -1.17314400 & -0.45282200 \\ \text { H } & -4.41272100 & -0.99798200 & -1.35280800 \\ \text { C } & -3.05151800 & -2.49339800 & -0.65241500 \\ \text { H } & -2.59952700 & -2.51048100 & -1.65342400 \\ \text { H } & -3.75437400 & -3.33187400 & -0.60823700 \\ \text { C } & -0.30768800 & 3.58087100 & -0.11676500 \\ \text { H } & 0.31351500 & 3.72683200 & 0.77082300 \\ \text { H } & 0.37706100 & 3.50980800 & -0.97059500 \\ \text { H } & -0.91154900 & 4.47829200 & -0.25964000 \\ \text { C } & 3.02667700 & 0.14055400 & 0.78906800 \\ \text { O } & 3.80013700 & 0.68911600 & 1.68263500 \\ \text { C } & -0.93501500 & -1.52051800 & 0.26074600 \\ \text { H } & -0.10012700 & -1.68597600 & 0.95823100 \\ \text { H } & -0.52846900 & -1.55357200 & -0.75713900 \\ \text { C } & 2.48188400 & -1.60987800 & -0.70067400 \\ \text { O } & 2.47138000 & 2.32584400 & 0.13523500 \\ \text { O } & 1.75340800 & -0.84051100 & -1.46444800 \\ \text { H } & 0.39771000 & 1.42636100 & -2.00469200 \\ \text { H } & 2.64430000 & 2.52958900 & -0.80529800 \\ \text { H } & 3.67040500 & 1.65910400 & 1.67702600\end{array}$

\begin{tabular}{|c|c|c|c|}
\hline & $\begin{array}{l}\text { f electron } \\
93.872689\end{array}$ & and thermal & Free Energie \\
\hline C & 2.83555800 & -0.10791800 & 0.39106000 \\
\hline $\mathbf{F}$ & 2.50072300 & 0.05782300 & 1.42682800 \\
\hline$C$ & 1.57104000 & -2.69316900 & -0.21143000 \\
\hline $\mathbf{H}$ & 1.92614800 & -2.84299000 & -1.23779800 \\
\hline $\mathbf{H}$ & 0.94396000 & -3.55806200 & 0.02745400 \\
\hline C & 4.57693900 & -1.74270900 & -0.74096600 \\
\hline $\mathbf{H}$ & 5.17652300 & -0.88717300 & -1.06183900 \\
\hline $\mathbf{H}$ & 5.27705600 & -2.52748200 & -0.44100200 \\
\hline $\mathbf{H}$ & 4.03592300 & -2.12384500 & -1.61101900 \\
\hline C & 1.85197100 & -0.11933300 & -1.97450400 \\
\hline $\mathbf{H}$ & 0.98747400 & -0.42096100 & -2.57294700 \\
\hline $\mathbf{H}$ & 2.16477600 & 0.87422200 & -2.30807100 \\
\hline $\mathbf{H}$ & 2.65781500 & -0.81105700 & -2.21357900 \\
\hline c & 1.54088100 & -0.15189800 & -0.46141800 \\
\hline c & 0.71458600 & 1.13136700 & -0.14618300 \\
\hline C & 2.86052000 & 2.36240400 & -0.03125900 \\
\hline $\mathbf{H}$ & 3.40360300 & 3.30519900 & 0.00506200 \\
\hline C & 3.67441600 & 1.10706900 & 0.00034700 \\
\hline H & 4.50233500 & 1.23382400 & 0.70778200 \\
\hline H & 4.14313700 & 0.96941400 & -0.98309800 \\
\hline C & 1.52392600 & 2.40964500 & -0.08752900 \\
\hline C & -0.59265000 & 1.20648900 & -0.97278800 \\
\hline $\mathbf{H}$ & -0.63470200 & 2.09254100 & -1.60893700 \\
\hline $\mathbf{H}$ & -0.68062000 & 0.34257400 & -1.62835500 \\
\hline 0 & 0.18752100 & 0.97858900 & 1.27487900 \\
\hline C & -1.77461800 & 1.20225700 & 0.01900500 \\
\hline C & -1.06932800 & 0.86435600 & 1.34134500 \\
\hline C & -3.32025200 & -0.87499300 & -0.46252600 \\
\hline 0 & -1.59757000 & 0.73483200 & 2.49100400 \\
\hline c & -2.51836600 & -3.23590200 & 0.13128300 \\
\hline $\mathbf{H}$ & -2.05361600 & -3.30813800 & -0.85624600 \\
\hline $\mathbf{F}$ & -1.90941100 & -3.73337900 & 0.88575400 \\
\hline $\mathbf{H}$ & -3.48833900 & -3.74203800 & 0.07008100 \\
\hline c & -4.37340100 & -1.45086300 & -1.38043600 \\
\hline $\mathrm{E}$ & -4.88107400 & -0.66065400 & -1.92981900 \\
\hline H & -3.92207400 & -2.13536200 & -2.10668600 \\
\hline
\end{tabular}




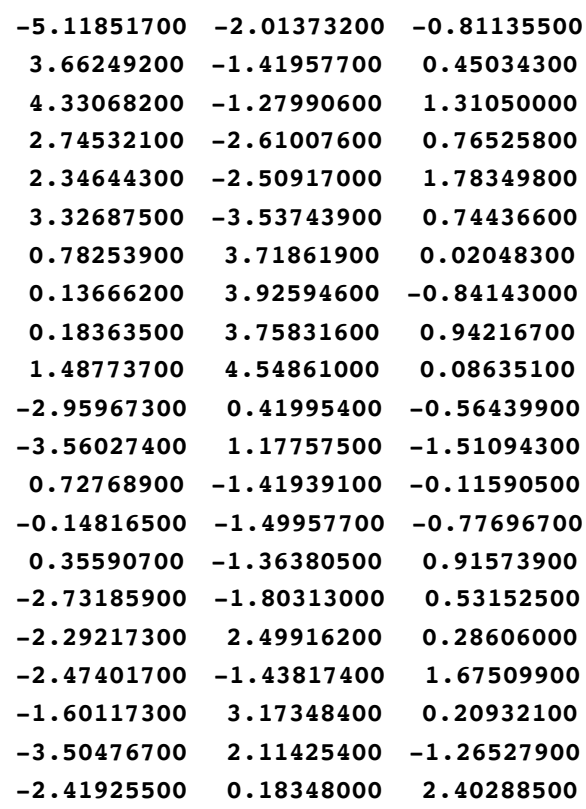

$-0.81135500$

0.45034300

1.31050000

0.76525800

1.78349800

0.74436600

0.02048300

$-0.84143000$

0.94216700

0.08635100

$-0.56439900$

$-1.51094300$

$-0.11590500$

$-0.77696700$

0.91573900

0.53152500

0.28606000

1.67509900

0.20932100

$-1.26527900$

2.40288500

TS_6b-7

Sum of electronic and thermal Free Energies $=-1193.870190$

\begin{tabular}{|c|c|c|c|}
\hline$C$ & -3.16006700 & 0.37158400 & -0.43116700 \\
\hline $\mathbf{H}$ & -2.75382300 & 0.45114000 & -1.45122500 \\
\hline & -2.97397000 & -2.52650200 & -0.01329500 \\
\hline & -3.40147400 & -2.61392500 & 0.99275600 \\
\hline & -2.70074700 & -3.54186000 & -0.31691700 \\
\hline & -5.40592100 & -0.55927100 & 0.59225600 \\
\hline & -5.66663700 & 0.43812100 & 0.95536000 \\
\hline & -6.33426500 & -1.02876600 & 0.25439700 \\
\hline & -5.04936100 & -1.14608200 & 1.44239700 \\
\hline & -2.30050100 & -0.13164300 & 1.93808300 \\
\hline & -1.54186800 & -0.66194300 & 2.52174300 \\
\hline & -2.35055500 & 0.89737700 & 2.30410100 \\
\hline & -3.25546000 & -0.60678900 & 2.15733800 \\
\hline & -1.99767400 & -0.20178700 & 0.42583900 \\
\hline & -0.75607900 & 0.69071500 & 0.18166700 \\
\hline & -2.28012400 & 2.64469300 & 0.14093400 \\
\hline & -2.42920900 & 3.72267200 & 0.17490200 \\
\hline & -3.50074300 & 1.79083700 & 0.01528800 \\
\hline & -4.19217800 & 2.25224800 & -0.69997200 \\
\hline & -4.02779000 & 1.79224200 & 0.97897100 \\
\hline & -1.02561400 & 2.18177200 & 0.21213700 \\
\hline & 0.50698700 & 0.28904900 & 1.01004100 \\
\hline & 0.75862100 & 1.04975400 & 1.74927300 \\
\hline & 0.33521500 & -0.65267200 & 1.53106100 \\
\hline & -0.29136800 & 0.47854400 & -1.29377300 \\
\hline & 1.63753400 & 0.07939900 & 0.00241200 \\
\hline & 0.98049400 & 0.47324300 & -1.31580200 \\
\hline & 3.61292000 & -1.23213500 & 0.16794800 \\
\hline & 1.76581500 & 0.91867300 & -2.16895600 \\
\hline & 4.58623400 & -2.35906100 & 0.32799900 \\
\hline & 4.90073300 & -2.46141800 & 1.37161200 \\
\hline & 4.15965000 & -3.31383400 & 0.01008500 \\
\hline & 5.48517700 & -2.18801500 & -0.27134400 \\
\hline & -4.41547700 & -0.52500700 & -0.58058500 \\
\hline & -4.96529100 & -0.08598500 & -1.42419700 \\
\hline
\end{tabular}

$\begin{array}{lrrr}\text { C } & -3.99940300 & -1.94532400 & -0.98563600 \\ \text { H } & -3.55763100 & -1.92466800 & -1.99103200 \\ \text { H } & -4.88450100 & -2.58774900 & -1.04177400 \\ \text { C } & 0.15530900 & 3.12187900 & 0.24050200 \\ \text { H } & 0.75101700 & 3.04538000 & 1.15541900 \\ \text { H } & 0.83219700 & 2.95789400 & -0.60731400 \\ \text { H } & -0.19110100 & 4.15355700 & 0.16329700 \\ \text { C } & 2.28742000 & -1.28513500 & 0.02173500 \\ \text { O } & 1.43740000 & -2.31953500 & -0.05620100 \\ \text { C } & -1.71218100 & -1.66097600 & 0.01394600 \\ \text { H } & -0.97357700 & -2.10661000 & 0.68879600 \\ \text { H } & -1.27370000 & -1.67645100 & -0.99160300 \\ \text { C } & 4.08317900 & 0.19830600 & 0.21994900 \\ \text { O } & 2.78208200 & 0.95736200 & -0.06377200 \\ \text { H } & 1.89637400 & -3.16741500 & 0.02162200 \\ \text { O } & 4.95766800 & 0.55485800 & -0.76992700 \\ \text { H } & 4.83080200 & 0.01627300 & -1.56497100 \\ \text { C } & 4.59964700 & 0.70034900 & 1.54451100 \\ \text { H } & 5.50506200 & 0.14684400 & 1.80352600 \\ \text { H } & 4.84915900 & 1.75960400 & 1.45918400 \\ \text { H } & 3.85081400 & 0.55362200 & 2.32530100 \\ \text { H } & 2.64974200 & 1.11832000 & -1.25233400\end{array}$

TS_7-8

Sum of electronic and thermal Free Energies $=-1193.899839$

\begin{tabular}{lrrr} 
C & 3.14362800 & 0.43173700 & 0.48807900 \\
H & 2.68977800 & 0.48021900 & 1.49006400 \\
C & 3.11257500 & -2.46582400 & 0.01809500 \\
H & 3.58132500 & -2.50949300 & -0.97247400 \\
H & 2.87918600 & -3.49958900 & 0.29151800 \\
C & 5.47686900 & -0.37586500 & -0.44494900 \\
H & 5.69857500 & 0.63790800 & -0.78813500 \\
H & 6.41293100 & -0.79808700 & -0.06791800 \\
H & 5.19182800 & -0.97293400 & -1.31494200 \\
C & 2.41571600 & -0.07525500 & -1.91866100 \\
H & 1.73969900 & -0.66962300 & -2.54121400 \\
H & 2.38389200 & 0.95796700 & -2.27644600 \\
H & 3.42148000 & -0.45284900 & -2.09676300 \\
C & 2.04740600 & -0.18194200 & -0.42451700 \\
C & 0.74670900 & 0.64489500 & -0.21373900 \\
C & 2.18041300 & 2.67222300 & -0.08129200 \\
H & 2.27866800 & 3.75662300 & -0.07774800 \\
C & 3.43578900 & 1.87110000 & 0.06991300 \\
H & 4.08344100 & 2.35066500 & 0.81373100 \\
H & 3.99319200 & 1.90603500 & -0.87570100 \\
C & 0.95544000 & 2.15084300 & -0.20738900 \\
C & -0.41316600 & 0.19690500 & -1.14960200 \\
H & -0.61692100 & 0.92293100 & -1.93727800 \\
H & -0.17173800 & -0.75672400 & -1.62000500 \\
O & 0.21772800 & 0.34710300 & 1.15207100 \\
C & -1.62638300 & -0.00781300 & -0.25810200 \\
C & -1.06517500 & 0.15211700 & 1.17486500 \\
C & -3.69666700 & -1.11322600 & -0.40240600 \\
H & -1.75243100 & 0.11118900 & 2.18096200 \\
- & -4.43145000 & -2.08435100 & -0.43747700 \\
\hline
\end{tabular}




$\begin{array}{rrrr}\text { H } & 4.92241900 & 0.03998300 & 1.55586000 \\ \text { C } & 4.06951500 & -1.85510400 & 1.04162500 \\ \text { H } & 3.58679700 & -1.87634700 & 2.02796600 \\ \text { H } & 4.98216000 & -2.45526300 & 1.12247800 \\ \text { C } & -0.27380400 & 3.02298500 & -0.24308700 \\ \text { H } & -0.84519500 & 2.92561400 & -1.17178800 \\ \text { H } & -0.95714100 & 2.78689200 & 0.58340200 \\ \text { H } & 0.00758400 & 4.07180000 & -0.13570500 \\ \text { C } & -2.36578900 & -1.31310800 & -0.38459600 \\ \text { O } & -1.62106400 & -2.41217200 & -0.41054200 \\ \text { C } & 1.81386500 & -1.65849400 & -0.03797600 \\ \text { H } & 1.11489900 & -2.13135500 & -0.73862700 \\ \text { H } & 1.35191100 & -1.70481300 & 0.95578400 \\ \text { C } & -3.86481600 & 0.33733900 & -0.28767700 \\ \text { O } & -2.68076300 & 0.95607600 & -0.42053700 \\ \text { O } & -4.20937800 & 0.46067000 & 1.48695700 \\ \text { H } & -4.50855000 & 1.33916200 & 1.77272000 \\ \text { C } & -5.03482800 & 1.08141800 & -0.83603300 \\ \text { H } & -5.96787100 & 0.65344600 & -0.46700600 \\ \text { H } & -4.97395300 & 2.14154900 & -0.58293800 \\ \text { H } & -5.00684200 & 0.98501500 & -1.92550400 \\ \text { H } & -3.30749600 & 0.27620100 & 1.91896900 \\ \text { H } & -2.15534300 & -3.21930400 & -0.39463700\end{array}$

IM1 '

Sum of electronic and thermal Free Energies $=-1270.264236$
$2.89662400-0.92568700 \quad 0.11000600$
$2.35641400-1.65485600-0.51363400$
$\begin{array}{llll}1.90782000 & 0.98979900 & -1.22302200\end{array}$
$\begin{array}{llll}1.36153300 & 0.33509900 & -1.91168000\end{array}$
$\begin{array}{llll}1.34610900 & 1.93171400 & -1.15977900\end{array}$
$3.29306700 \quad 1.25582300 \quad-1.81544800$
$3.84845700 \quad 1.96762800 \quad-1.19253200$
$3.18073500 \quad 1.73006200 \quad-2.79571200$
$5.38682700-0.05808000 \quad 0.20032000$
$5.50490900-0.47232600 \quad 1.20486200$
$6.33457600-0.21305400-0.32369600$
$\begin{array}{llll}5.25293700 & 1.02258300 & 0.29388500\end{array}$
$2.44221200 \quad 1.33768600 \quad 1.22433300$
$1.90265200 \quad 2.28570800 \quad 1.13247600$
$2.29969000 \quad 0.96602800 \quad 2.24326500$
$3.50069400 \quad 1.56428200 \quad 1.10312100$
$\begin{array}{llll}1.97125800 & 0.31921600 & 0.16560700\end{array}$
$0.55842100-0.17659800 \quad 0.58360300$
$1.69052100 \quad-1.74626500 \quad 2.14932200$
$\begin{array}{llll}1.64161800 & -2.44356900 & 2.98445600\end{array}$
$3.02635000-1.54751900 \quad 1.49991200$
$3.54144500 \quad-2.51326700 \quad 1.42872500$
$3.65081800-0.92584300 \quad 2.15538500$
$0.56331200-1.14095600 \quad 1.76106800$
$\begin{array}{lll}-0.44117100 & 1.00969800 & 0.80586000\end{array}$
$\begin{array}{llll}-0.64379200 & 1.17367600 & 1.86578600\end{array}$
$\begin{array}{lll}-0.05537800 & 1.94115200 & 0.39053100\end{array}$
$\begin{array}{llll}-0.02860800 & -0.96581900 & -0.53513200\end{array}$
$\begin{array}{llll}-1.66786900 & 0.58154800 & 0.04183600\end{array}$
$-1.31298400 \quad-0.62118800-0.73924200$
$\begin{array}{lll}-3.07654700 & 0.66503700 & 0.46671000\end{array}$
$-2.31588600-1.66998000-1.02375500$
$-3.57726100-1.41706900-0.65571900$
$\begin{array}{llll}-3.91676100 & -0.22271200 & 0.04000800\end{array}$

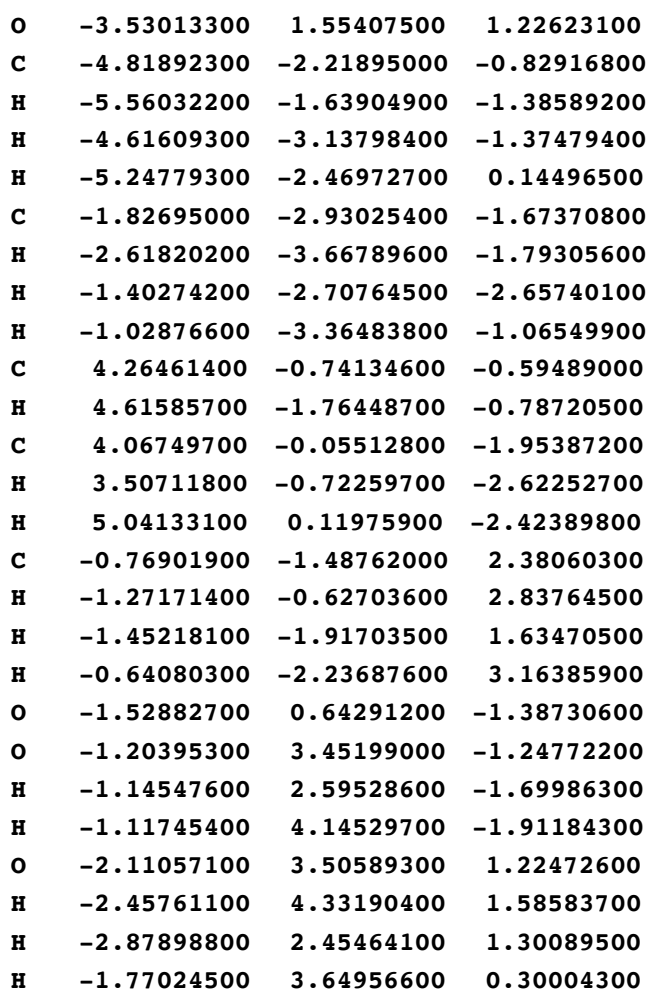

IM2 $a$

Sum of electronic and thermal Free Energies $=-1270.298274$

\begin{tabular}{lrrr} 
C & 2.87853600 & -0.84921200 & 0.29371400 \\
H & 2.32351500 & -1.77006800 & 0.05583300 \\
C & 1.61941600 & 0.41293200 & -1.51349000 \\
H & 1.04274300 & -0.46045200 & -1.84444300 \\
H & 1.00631400 & 1.29681600 & -1.72584000 \\
C & 2.90856400 & 0.45658400 & -2.33629700 \\
H & 3.48233200 & 1.36065900 & -2.09920300 \\
H & 2.65257100 & 0.52682100 & -3.39785700 \\
C & 5.30338500 & 0.03147000 & -0.26990200 \\
H & 5.55059400 & 0.04514900 & 0.79477900 \\
H & 6.19707200 & -0.30823100 & -0.80129900 \\
H & 5.12188900 & 1.06080200 & -0.58904200 \\
C & 2.42144000 & 1.66479900 & 0.52935700 \\
H & 1.84317400 & 2.50145000 & 0.12642900 \\
H & 2.38937200 & 1.72341700 & 1.62118800 \\
H & 3.45552700 & 1.81581500 & 0.22345600 \\
C & 1.89230300 & 0.31290700 & 0.00177400 \\
C & 0.57963400 & 0.01763700 & 0.77015300 \\
C & 1.96676300 & -0.85712400 & 2.62986000 \\
H & 2.05842500 & -1.18161500 & 3.66537100 \\
C & 3.20195000 & -0.90702900 & 1.78538500 \\
H & 3.76143700 & -1.82231800 & 2.01290200 \\
H & $\mathbf{3} .85788100$ & -0.07711300 & 2.08137900 \\
C & 0.76919000 & -0.43563800 & 2.20525100 \\
C & -0.52361700 & 1.09335000 & 0.59266600 \\
H & -0.89667100 & 1.45128100 & 1.55244100 \\
H & -0.15195200 & 1.95974800 & 0.04828500 \\
C & -0.06801200 & -1.23368200 & 0.14243800 \\
\hline & -1.63972900 & 0.43011000 & -0.21368400 \\
C.28515500 & -1.03394400 & -0.16247700
\end{tabular}




$\begin{array}{lrrr}\text { C } & -3.06701300 & 0.63198200 & 0.26436700 \\ \text { C } & -2.22048100 & -2.02605500 & -0.48731400 \\ \text { C } & -3.52961700 & -1.59088600 & -0.52131400 \\ \text { O } & -3.93991700 & -0.35405000 & -0.16531700 \\ \text { O } & -3.51267000 & 1.56976200 & 0.84937900 \\ \text { C } & -4.69124500 & -2.44648000 & -0.86532100 \\ \text { H } & -5.33787800 & -1.90114800 & -1.55854600 \\ \text { H } & -4.38247700 & -3.39148300 & -1.30690600 \\ \text { H } & -5.27819600 & -2.63833900 & 0.04022500 \\ \text { C } & -1.81711900 & -3.45960300 & -0.72134100 \\ \text { H } & -2.41243900 & -4.14874500 & -0.11917000 \\ \text { H } & -1.92628900 & -3.73077200 & -1.77488800 \\ \text { H } & -0.76944900 & -3.59232100 & -0.44811600 \\ \text { C } & 4.14294900 & -0.91872200 & -0.60079500 \\ \text { H } & 4.53378800 & -1.93338200 & -0.44343800 \\ \text { C } & 3.74627000 & -0.79612700 & -2.07889000 \\ \text { H } & 3.15935500 & -1.67652100 & -2.37476100 \\ \text { H } & 4.64656600 & -0.79306800 & -2.70256800 \\ \text { C } & -0.44856900 & -0.47141800 & 3.09475200 \\ \text { H } & -0.74688200 & 0.52219200 & 3.44433700 \\ \text { H } & -1.31703000 & -0.90952800 & 2.58095100 \\ \text { H } & -0.25649900 & -1.08688100 & 3.97502100 \\ \text { O } & -1.66346700 & 0.72256500 & -1.58850400 \\ \text { O } & -1.07412100 & 3.29025600 & -1.64267300 \\ \text { H } & -1.48492500 & 1.69568700 & -1.70782900 \\ \text { H } & -1.16225300 & 3.86043300 & -2.41401100 \\ \text { O } & -1.98481000 & 4.00211900 & 0.81240200 \\ \text { H } & -2.09554300 & 4.85641500 & 1.24386000 \\ \text { H } & -2.73709700 & 3.44764600 & 1.06342800 \\ \text { H } & -1.43014600 & 3.76468200 & -0.86250000\end{array}$

IM2b '

Sum of electronic and thermal Free Energies $=-1270.284240$

$\begin{array}{lrrr}\text { C } & 2.61674000 & -0.61191200 & -0.83784100 \\ \text { H } & 2.00198600 & -0.07029700 & -1.57396800 \\ \text { C } & 1.78638900 & 0.92615700 & 0.99079700 \\ \text { H } & 1.18075300 & 1.53185700 & 0.30660200 \\ \text { H } & 1.30091800 & 0.98378600 & 1.97399600 \\ \text { C } & 3.17829000 & 1.55542800 & 1.06324700 \\ \text { H } & 3.80643700 & 1.02869100 & 1.79175400 \\ \text { H } & 3.07799500 & 2.58470800 & 1.41854600 \\ \text { C } & 5.17734800 & -0.59061600 & -0.20127700 \\ \text { H } & 5.26395800 & -1.63994000 & -0.49474900 \\ \text { H } & 6.09952300 & -0.09336800 & -0.51573300 \\ \text { H } & 5.15323800 & -0.54523700 & 0.89024700 \\ \text { C } & 2.40711500 & -1.46200800 & 1.57604000 \\ \text { H } & 1.94794800 & -1.27469500 & 2.55129400 \\ \text { H } & 2.26596500 & -2.51948900 & 1.33468100 \\ \text { H } & 3.47619000 & -1.29075400 & 1.69140800 \\ \text { C } & 1.82022700 & -0.53390700 & 0.49226500 \\ \text { C } & 0.38385300 & -1.03686900 & 0.20741400 \\ \text { C } & 1.36674800 & -2.72703200 & -1.32143500 \\ \text { H } & 1.26315400 & -3.63544100 & -1.91271500 \\ \text { C } & 2.70483900 & -2.05715300 & -1.32279700 \\ \text { H } & 3.12342900 & -2.08727400 & -2.33599800 \\ \text { H } & 3.39227300 & -2.64576200 & -0.70052900 \\ \text { C } & 0.29686300 & -2.28833600 & -0.64701500 \\ \text { C } & -0.53252600 & -1.08881700 & 1.45453500 \\ \text { H } & -0.75548600 & -2.11333600 & 1.75518600 \\ & & & \end{array}$

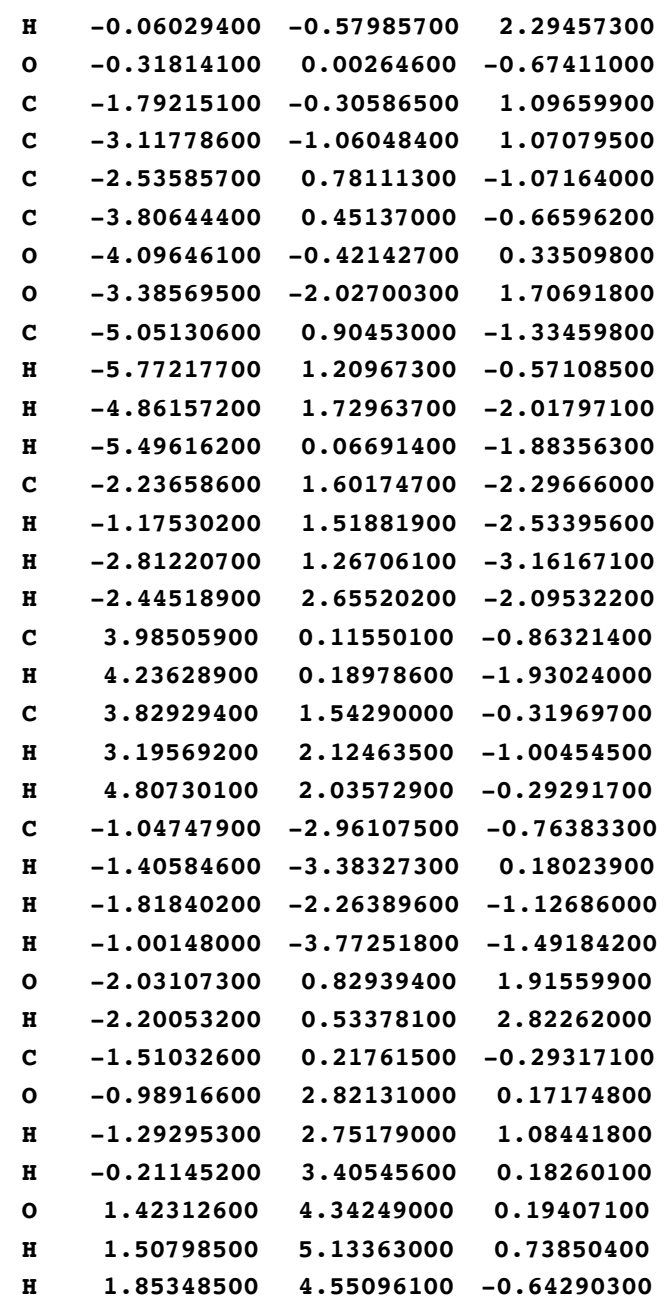

IM3 '

Sum of electronic and thermal Free Energies $=-1270.276843$

$\begin{array}{lrrr}\text { C } & 2.65184800 & -0.16017900 & -0.86881200 \\ \text { H } & 2.06910600 & 0.62358200 & -1.38333900 \\ \text { C } & 1.99311700 & 0.68902000 & 1.44579200 \\ \text { H } & 1.39326600 & 1.54376300 & 1.11418300 \\ \text { H } & 1.58651100 & 0.39219400 & 2.41889900 \\ \text { C } & 3.43406300 & 1.17025400 & 1.63509100 \\ \text { H } & 4.04424700 & 0.38071900 & 2.08786500 \\ \text { H } & 3.44150400 & 2.00296100 & 2.34487600 \\ \text { C } & 5.21937000 & -0.51317500 & -0.39903400 \\ \text { H } & 5.25159600 & -1.35993900 & -1.08842200 \\ \text { H } & 6.16686600 & 0.02244600 & -0.50639200 \\ \text { H } & 5.19195600 & -0.90718800 & 0.61936200 \\ \text { C } & 2.40006600 & -1.76933900 & 1.11247900 \\ \text { H } & 1.93180000 & -1.92181600 & 2.08867900 \\ \text { H } & 2.21222600 & -2.65930900 & 0.50759500 \\ \text { H } & 3.47380400 & -1.70288200 & 1.28211700 \\ \text { C } & 1.87587600 & -0.47976300 & 0.43996200 \\ \text { C } & 0.39125300 & -0.76721300 & 0.05766200 \\ \text { C } & 1.25820700 & -1.93555300 & -1.95643100 \\ \text { H } & 1.09186400 & -2.60486700 & -2.79844900 \\ \text { C } & 2.63421800 & -1.36793200 & -1.80238900 \\ \text { H } & 3.02534300 & -1.08401000 & -2.78672600\end{array}$




\begin{tabular}{|c|c|c|}
\hline 3.30247800 & -2.15702300 & -1.43406100 \\
\hline 0.22880600 & -1.68479100 & -1.13954500 \\
\hline-0.46540500 & -1.14353100 & 1.27502800 \\
\hline-0.66205500 & -2.21624200 & 1.3176020 \\
\hline 0.04710200 & -0.86204000 & 2.19507600 \\
\hline-0.32215300 & 0.51884600 & -0.39747200 \\
\hline-1.77488300 & -0.33582000 & 1.27307600 \\
\hline-2.98141800 & -1.20344700 & 0.89335400 \\
\hline-2.62582400 & 1.21812600 & -0.61304400 \\
\hline-3.68186200 & 0.40504900 & -0.74903900 \\
\hline-3.84441700 & -0.77798800 & -0.03463800 \\
\hline-3.18011900 & -2.22133800 & 1.49625400 \\
\hline-4.86515900 & 0.55093600 & -1.64684600 \\
\hline-4.92482000 & -0.32086200 & -2.30498900 \\
\hline-5.77573400 & 0.56632100 & -1.04138300 \\
\hline-4.82552300 & 1.45035800 & -2.25465500 \\
\hline-2.44804300 & 2.49570800 & -1.38949500 \\
\hline-3.31839100 & 2.72732600 & -1.99871700 \\
\hline-2.29141900 & 3.33325500 & -0.70429500 \\
\hline-1.58247600 & 2.43356700 & -2.05645700 \\
\hline 4.06788200 & 0.45312700 & -0.71115500 \\
\hline 4.29815500 & 0.87879700 & -1.69766100 \\
\hline 4.03902000 & 1.60490600 & 0.30204400 \\
\hline 3.44319200 & 2.44071700 & -0.09836200 \\
\hline 5.05028000 & 1.99895500 & 0.44515300 \\
\hline-1.14680300 & -2.23075200 & -1.41457200 \\
\hline-1.52296300 & -2.86957700 & -0.60872600 \\
\hline-1.86423100 & -1.41505100 & -1.57051900 \\
\hline-1.14160000 & -2.82747800 & -2.32805300 \\
\hline-2.07041900 & 0.17740300 & 2.55783800 \\
\hline-2.38116600 & -0.53806800 & 3.13320100 \\
\hline-1.53818800 & 0.90147200 & 0.37377100 \\
\hline 0.65504400 & 2.74627100 & -0.93107000 \\
\hline 0.23172700 & 1.37399100 & -0.64138600 \\
\hline 0.30268000 & 3.32771900 & -0.23949800 \\
\hline 1.57965500 & 2.98127900 & -1.08487400 \\
\hline-1.17600500 & 2.02276400 & 1.09916000 \\
\hline-1.33729300 & 1.85812300 & 2.0444050 \\
\hline
\end{tabular}

IM4a'

Sum of electronic and thermal Free Energies $=-1270.277523$
$2.60824700 \quad 0.08632700$
0.42155100
$2.09357100 \quad 0.18383600 \quad 1.39026400$
$0.90221100-1.58191800-0.39597600$
$0.34325300-1.57741200 \quad 0.54796700$
$0.19782500-1.82160600-1.20456500$
$1.96381900-2.67920300-0.31416400$
$2.51430900-2.75132600 \quad-1.26021000$
$1.47525300-3.64909500-0.16954100$
$4.78001800-1.19997400-0.36875400$
$5.27015400-0.25035400-0.59777000$
$5.54371500-1.86004200 \quad 0.05299600$
$4.46003300-1.65239200-1.31098400$
$2.03806200-0.06157200 \quad-2.06155600$
$1.41066900-0.59855600 \quad-2.78041800$
$2.11346900 \quad 0.98025800 \quad-2.38914000$
$3.03418800-0.49595000 \quad-2.13927600$
$1.48990000-0.17691300-0.62241300$
$\begin{array}{llll}0.36929800 & 0.90919700 & -0.39628500\end{array}$

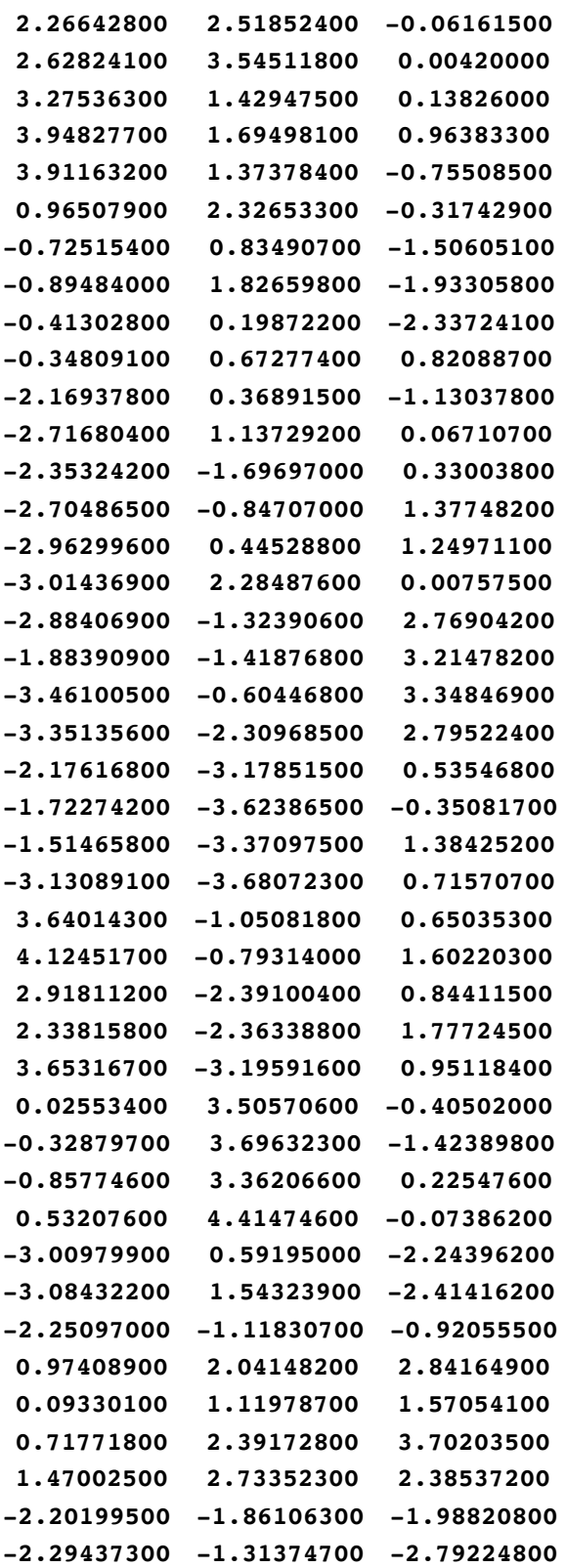

IM4 b '

Sum of electronic and thermal Free Energies $=-1270.274939$

\begin{tabular}{lrrr}
\hline C & 2.83594300 & -0.02140800 & 0.25617000 \\
H & 2.44878600 & 0.19708500 & 1.26611000 \\
C & 2.00156200 & -2.81519100 & -0.05694000 \\
H & 2.40151800 & -3.02373700 & -1.05627200 \\
H & 1.50531000 & -3.73425400 & 0.27189100 \\
C & 4.83415500 & -1.47922800 & -0.66901500 \\
H & 5.31665800 & -0.58244200 & -1.06510300 \\
H & 5.62817100 & -2.12569200 & -0.28420400 \\
H & 4.37282000 & -2.01255600 & -1.50393700 \\
C & 1.91741200 & -0.42992600 & -2.10037700 \\
H & 1.09558300 & -0.87000100 & -2.67319400 \\
H & 2.13453100 & 0.55414300 & -2.52557400 \\
H & 2.79020500 & -1.05881200 & -2.26858600 \\
C & 1.58025500 & -0.34693500 & -0.59691000
\end{tabular}




\begin{tabular}{|c|c|c|c|}
\hline C & 0.55635100 & 0.82469200 & -0.41698100 \\
\hline C & 2.51371200 & 2.36098500 & -0.44041200 \\
\hline $\mathbf{H}$ & 2.91437300 & 3.36998200 & -0.52192500 \\
\hline C & 3.50099500 & 1.25410600 & -0.24979700 \\
\hline $\mathbf{F}$ & 4.28209600 & 1.57445000 & 0.45037700 \\
\hline F & 4.01426800 & 1.07718600 & -1.20424000 \\
\hline & 1.18605600 & 2.20948900 & -0.52565900 \\
\hline & -0.69694000 & 0.62315300 & -1.31561200 \\
\hline F & -0.69158200 & 1.30004000 & -2.17270600 \\
\hline F & -0.71221400 & -0.39641600 & -1.70186100 \\
\hline & -0.02397700 & 0.74723900 & 0.95206300 \\
\hline & -1.96582200 & 0.86548100 & -0.48060900 \\
\hline & -1.59154000 & 0.66167200 & 1.00510800 \\
\hline & -3.22523900 & -1.34251400 & -0.36476400 \\
\hline & -2.09379500 & 1.40118200 & 1.85693500 \\
\hline & -2.50124300 & -2.98216100 & 1.41971100 \\
\hline H & -2.15034900 & -3.74019600 & 0.71088800 \\
\hline & -1.88396200 & -2.99662100 & 2.3 \\
\hline & -3.53634300 & -3.23249100 & 1.67230700 \\
\hline & -4.23677500 & -2.32437900 & -0.89770300 \\
\hline H & -4.66748800 & -1.94974400 & -1.82605200 \\
\hline & -3.77626600 & -3.29357000 & -1.10589800 \\
\hline & -5.05430100 & -2.48082300 & -0.18798000 \\
\hline & 3.85280800 & -1.17415100 & 0.47268800 \\
\hline H & 4.47239500 & -0.83655800 & 1.31479400 \\
\hline$c$ & 3.12580900 & -2.45160600 & 0.91259900 \\
\hline $\mathbf{H}$ & 2.69131200 & -2.30278900 & 1.91064100 \\
\hline $\mathbf{H}$ & 3.84384600 & -3.27384400 & 0.99942800 \\
\hline C & 0.28084700 & 3.40897000 & -0.63841900 \\
\hline I & -0.34439100 & 3.38931800 & -1.53452000 \\
\hline H & -0.39505600 & 3.47884500 & 0.22085900 \\
\hline H & 0.88134200 & 4.31990900 & -0.65971100 \\
\hline C & -3.07100600 & -0.08407900 & -0.89495900 \\
\hline C & -3.85999700 & 0.34834600 & -1.84390800 \\
\hline C & 0.97609800 & -1.68104200 & -0.10868900 \\
\hline H & 0.13230900 & -1.97768700 & -0.74881900 \\
\hline H & 0.58389800 & -1.54267700 & 0.90455200 \\
\hline C & -2.44683500 & -1.64072100 & 0.77536100 \\
\hline 0 & -2.44938700 & 2.17029900 & -0.65590900 \\
\hline 0 & -1.65744900 & -0.80637000 & 1.36647400 \\
\hline H & 0.24960900 & 1.51495300 & 1.62264800 \\
\hline H & -2.75682000 & 2.48288700 & 0.21672800 \\
\hline H & -3.69195200 & 1.30148200 & -1.98703000 \\
\hline 0 & 0.23368000 & 2.54192400 & 2.61624300 \\
\hline $\mathrm{F}$ & -0.68435500 & 2.47358800 & 2.93500200 \\
\hline & 0.83383400 & 2.56485100 & 3.37128900 \\
\hline
\end{tabular}

IM5 '

Sum of electronic and thermal Free Energies $=-1270.304276$

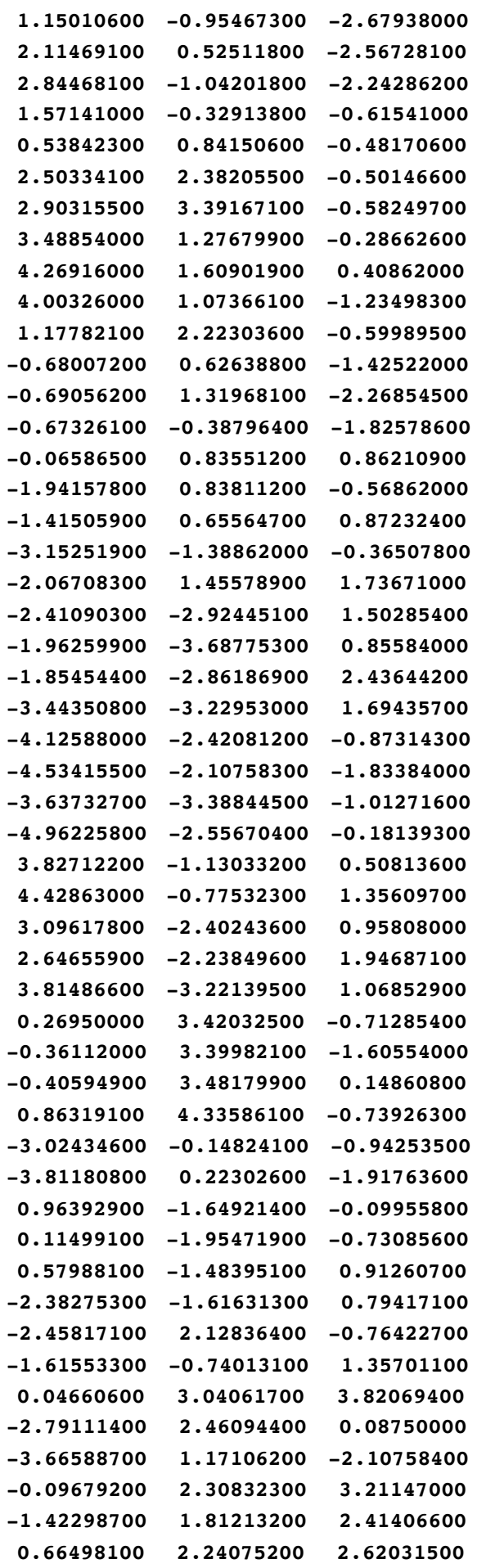

$\begin{array}{lrrr}\text { C } & 2.81401900 & 0.01760400 & 0.24818900 \\ \text { H } & 2.40951100 & 0.26593400 & 1.24338900 \\ \text { C } & 1.98597100 & -2.78430600 & -0.02127400 \\ \text { H } & 2.39877300 & -3.00615800 & -1.01262400 \\ \text { H } & 1.48760200 & -3.69959000 & 0.31559300 \\ \text { C } & 4.83364600 & -1.45842700 & -0.60549200 \\ \text { H } & 5.31937600 & -0.56936800 & -1.01492500 \\ \text { H } & 5.62301700 & -2.09039100 & -0.18822100 \\ \text { H } & 4.39208200 & -2.01617300 & -1.43541400 \\ \text { C } & 1.93857000 & -0.45302500 & -2.10976500\end{array}$

IM6a '

Sum of electronic and thermal Free Energies $=-1270.291251$

$\begin{array}{llll}\text { C } & 2.91930600 & -0.11608400 & 0.34926500 \\ \text { H } & 2.47021000 & -0.17232900 & 1.35435300 \\ \text { C } & 1.96150600 & -2.62120600 & -0.83857200 \\ \text { H } & 2.42374900 & -2.55441400 & -1.83054500 \\ \text { H } & 1.39142800 & -3.55561600 & -0.82984700 \\ \text { C } & 4.89991300 & -1.37525400 & -0.86079000\end{array}$




\begin{tabular}{|c|c|c|c|}
\hline 1 & 5.46076500 & -0.44058600 & -0.93727400 \\
\hline $\mathbf{H}$ & 5.62486200 & -2.16622200 & -0.64807100 \\
\hline $\mathbf{H}$ & 4.47410800 & -1.59633600 & -1.84269500 \\
\hline & 2.15546300 & 0.26206500 & -2.07060800 \\
\hline $\mathbf{H}$ & 1.34970000 & 0.08327100 & -2.78867900 \\
\hline & 2.46826700 & 1.30563100 & -2.16617500 \\
\hline & 2.99468800 & -0.36076200 & -2.37582700 \\
\hline & 1.71577100 & -0.08362900 & -0.63102000 \\
\hline & 0.75080900 & 1.05377200 & -0.18251400 \\
\hline & 2.77511900 & 2.37691500 & 0.39101200 \\
\hline & 3.22993900 & 3.33257500 & 0.64579700 \\
\hline & 3.68802600 & 1.19890800 & 0.28587400 \\
\hline & 4.43116600 & 1.24005400 & 1.09136300 \\
\hline & 4.25896000 & 1.27989400 & -0.64870600 \\
\hline & 1.45032800 & 2.34879500 & 0.19896300 \\
\hline & -0.45683400 & 1.23437100 & -1.13994400 \\
\hline & -0.37788700 & 2.15206400 & -1.72574100 \\
\hline & -0.51855200 & 0.39422900 & -1.83038200 \\
\hline & 0.05512900 & 0.63529700 & 1.11607400 \\
\hline & -1.73240400 & 1.26269300 & -0.27855300 \\
\hline & -1.20935800 & 0.79676700 & 1.06441900 \\
\hline & -3.31420800 & -0.79285700 & -0.79652800 \\
\hline & -1.94100700 & 0.84215000 & 2.09993700 \\
\hline & -3.31074400 & -3.07902900 & 0.34021900 \\
\hline & -3.41413700 & -3.58812500 & -0.62194600 \\
\hline & -2.68880800 & -3.67256600 & 1.00886400 \\
\hline & -4.31625100 & -2.98538400 & 0.76377300 \\
\hline C & -4.46628200 & -1.28644500 & -1.64052900 \\
\hline & -4.94118300 & -0.46310500 & -2.17020000 \\
\hline & -4.12614700 & -2.01248800 & -2.38629600 \\
\hline & -5.22442700 & -1.77487000 & -1.02355600 \\
\hline & 3.85339400 & -1.35247000 & 0.26346000 \\
\hline & 4.42541200 & -1.32679200 & 1.20111300 \\
\hline & 3.02839100 & -2.64606500 & 0.25522300 \\
\hline & 2.52882000 & -2.76960200 & 1.22648700 \\
\hline & 9300100 & -3.50751000 & 0.13073200 \\
\hline & 726800 & 3.58425700 & 0.42616100 \\
\hline & 0.04483900 & 3.8 & -0.45423900 \\
\hline & -0.10507800 & 3.43982800 & 1.23768800 \\
\hline & 1.26521500 & 4.41492600 & 0.71008500 \\
\hline & -2.87935200 & 0.48598500 & -0.94260800 \\
\hline & -3.47140000 & 1.24931800 & -1.87409700 \\
\hline & 1.00535800 & -1.45084200 & -0.60371600 \\
\hline & 0.19117400 & -1.47959200 & -1.33969800 \\
\hline $\mathbf{H}$ & 0.54268800 & -1.59344600 & 0.37797600 \\
\hline $\mathbf{C}$ & -2.70005100 & -1.71661900 & 0.16427900 \\
\hline 0 & -2.16556300 & 2.60454500 & -0.11104500 \\
\hline & -1.70590000 & -1.40854500 & 0.83257600 \\
\hline & -0.37362100 & -1.03305200 & 4.10038300 \\
\hline & -2.82249800 & 2.65486400 & 0.60219200 \\
\hline & -3.22224800 & 2.18058800 & -1.75530800 \\
\hline & -1.14599700 & -0.97040300 & 3.52580300 \\
\hline & -1.55418500 & 0.21352400 & 2.85886200 \\
\hline & -1.09457200 & -1.64886400 & 2.83056900 \\
\hline
\end{tabular}

IM6b'

Sum of electronic and thermal Free Energies $=-1270.299427$

C $\quad 3.30309900 \quad-0.28630400 \quad-0.54601900$

H $\quad 2.82461100 \quad-0.51537700 \quad-1.51076600$

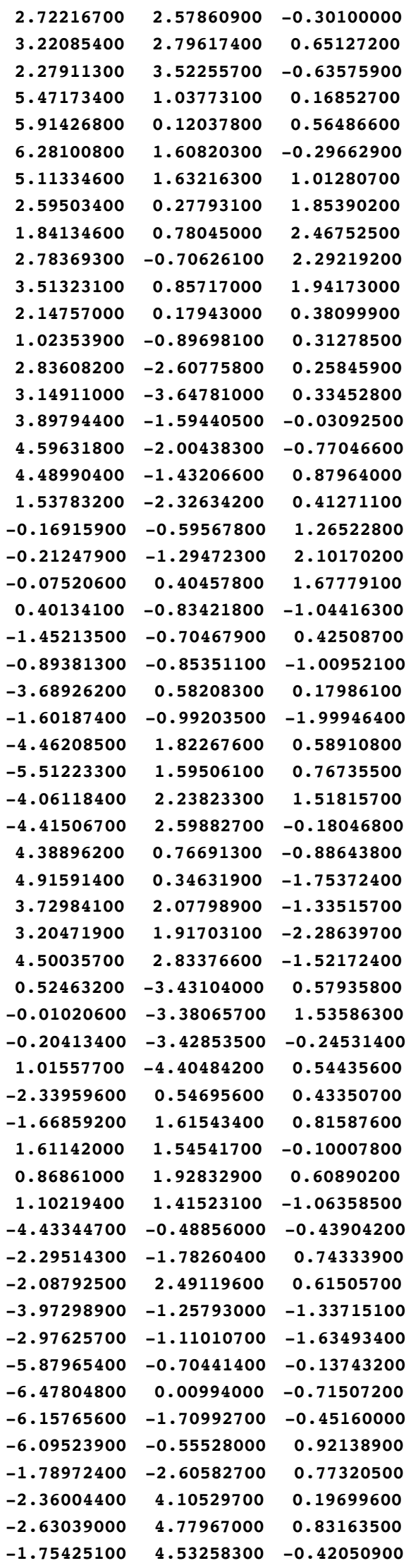

IM7 '

Sum of electronic and thermal Free Energies 
IM8 '

Sum of electronic and thermal Free Energies $=-1270.322170$

\begin{tabular}{|c|c|c|c|}
\hline & -3.35852900 & -0.35460700 & 0.50009500 \\
\hline & -2.90301100 & -0.47639000 & 1.49491800 \\
\hline & -3.05752800 & 2.53835000 & 0.10633500 \\
\hline & -3.52907900 & 2.65016500 & -0.87768700 \\
\hline & -2.72651100 & 3.53887300 & 0.40258600 \\
\hline & -5.61375100 & 0.69449400 & -0.38216300 \\
\hline & -5.94270400 & -0.28501800 & -0.73832200 \\
\hline & -6.49707500 & 1.20470000 & 0.01315500 \\
\hline & -5.27755200 & 1.27309000 & -1.24633600 \\
\hline & -2.59971000 & 0.14606300 & -1.89850300 \\
\hline & -1.87035700 & 0.68488100 & -2.51180400 \\
\hline & -2.67304500 & -0.87583800 & -2.28161300 \\
\hline & -3.56327300 & 0.62715500 & -2.06069300 \\
\hline & -2.21434400 & 0.17681200 & -0.40553300 \\
\hline & -0.99471000 & -0.77602800 & -0.22044400 \\
\hline & -2.61950000 & -2.65877700 & -0.14508400 \\
\hline & -2.82229800 & -3.72815700 & -0.17327100 \\
\hline & -3.79115000 & -1.74575500 & 0.04280400 \\
\hline & -4.47598200 & -2.18272700 & 0.77964600 \\
\hline & -4.35741900 & -1.69694400 & -0.89694000 \\
\hline & -1.35050700 & -2.25509500 & -0.26426800 \\
\hline & 0.18890300 & -0.40578300 & -1.16346400 \\
\hline & 0.30623300 & -1.11012600 & -1.98770800 \\
\hline & 0.03750100 & 0.58996900 & -1.58175700 \\
\hline & -0.43666900 & -0.55944500 & 1.13039800 \\
\hline & 1.42858400 & -0.35888300 & -0.28567900 \\
\hline & 0.86900000 & -0.43628600 & 1.16644000 \\
\hline & 3.63200800 & 0.41757400 & -0.57819600 \\
\hline & 1.54832100 & -0.38058200 & 2.15740300 \\
\hline & 4.89914900 & 1.20833700 & -0.64800100 \\
\hline & 5.51609400 & 0.88970200 & -1.49184100 \\
\hline & 4.69366800 & 2.27340900 & -0.76278100 \\
\hline & 5.46486300 & 1.05936400 & 0.27711000 \\
\hline 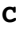 & -4.55978500 & 0.59806800 & 0.73070400 \\
\hline & -5.08171200 & 0.17485000 & 1.59982300 \\
\hline & -4.05861300 & 1.99399700 & 1.12500600 \\
\hline & -3.56636000 & 1.94267900 & 2.10548400 \\
\hline $\mathrm{H}$ & -4.90956600 & 2.67532500 & 1.23224400 \\
\hline & -0.21362100 & -3.24213400 & -0.33519500 \\
\hline & 0.36177200 & -3.16946600 & -1.26388400 \\
\hline & 0.49053900 & -3.10203800 & 0.49472600 \\
\hline $\mathbf{H}$ & -0.59732800 & -4.26110000 & -0.26305700 \\
\hline & 2.32659000 & 0.83236500 & -0.42087500 \\
\hline & 1.77969100 & 2.00350500 & -0.33289500 \\
\hline & -1.84108500 & 1.61444700 & 0.01743600 \\
\hline & -1.10647200 & 2.03975800 & -0.67799200 \\
\hline & -1.36880400 & 1.59019300 & 1.00688500 \\
\hline & 3.55529600 & -0.98426300 & -0.57192000 \\
\hline & 2.33294300 & -1.45938500 & -0.45487000 \\
\hline & 4.38436600 & -0.80119400 & 2.00426900 \\
\hline & 4.89301900 & -1.15005100 & 2.74409600 \\
\hline & 4.64839400 & -1.95877100 & -0.74166500 \\
\hline & 5.56250600 & -1.56183700 & -0.29981500 \\
\hline & 4.38318000 & -2.90553600 & -0.27145900 \\
\hline & 4.81008400 & -2.12327100 & -1.81406000 \\
\hline & 3.48911600 & -0.63733100 & 2.34028800 \\
\hline & 2.40436700 & 2.79175900 & -0.33773900 \\
\hline
\end{tabular}




$\begin{array}{rrrr}\text { O } & 3.23673600 & 4.14437700 & -0.29636100 \\ \text { H } & 3.38866700 & 4.57444300 & 0.55485400 \\ \text { H } & 3.05329500 & 4.84254900 & -0.93757500\end{array}$

TS_1'-2a'

Sum of electronic and thermal Free Energies $=-1270.260674$

\begin{tabular}{|c|c|c|c|}
\hline & 2.88746400 & -0.93572200 & -0.03042300 \\
\hline & 2.32810300 & -1.56214800 & -0.74253300 \\
\hline & 1.84985600 & 1.16016900 & -1.01067300 \\
\hline & 1.27647900 & 0.62163700 & -1.77533100 \\
\hline & 1.29787900 & 2.07996600 & -0.77776500 \\
\hline & 3.21272300 & 1.52116300 & -1.60484500 \\
\hline & 3.78945900 & 2.13087900 & -0.89883900 \\
\hline & 3.06466000 & 2.14187000 & -2.49439500 \\
\hline & 5.37675800 & -0.07331900 & 0.10943500 \\
\hline & 5.53091700 & -0.63911900 & 1.03178500 \\
\hline & 6.30607000 & -0.13657200 & -0.46426400 \\
\hline & 5.24341800 & 0.97810000 & 0.37632300 \\
\hline & 2.46669000 & 1.12312600 & 1.44062300 \\
\hline & 1.91488500 & 2.06492400 & 1.52252800 \\
\hline & 2.36859700 & 0.59219200 & 2.39173500 \\
\hline & 3.51721400 & 1.38218900 & 1.31555200 \\
\hline & 1.96267400 & 0.27935400 & 0.25159900 \\
\hline & 0.56822200 & -0.28184000 & 0.63799800 \\
\hline & 1.75160800 & -2.07452800 & 1.89149100 \\
\hline & 1.73148100 & -2.89416400 & 2.60809100 \\
\hline & 3.06479200 & -1.76741000 & 1.23887100 \\
\hline & 3.57797300 & -2.70668800 & 0.99948400 \\
\hline & 3.71010900 & -1.25319600 & 1.96363300 \\
\hline & 0.61009300 & -1.42391300 & 1.64192700 \\
\hline & -0.44956800 & 0.82998200 & 1.05712900 \\
\hline & -0.65831100 & 0.81216400 & 2.12773600 \\
\hline & -0.07798500 & 1.82640300 & 0.81274000 \\
\hline & -0.05535400 & -0.90212200 & -0.58312400 \\
\hline & -1.67820600 & 0.51013300 & 0.23584000 \\
\hline & -1.32969100 & -0.57921300 & -0.65576400 \\
\hline & -3.08884100 & 0.58702700 & 0.72486200 \\
\hline & -2.34504600 & -1.49966800 & -1.17166400 \\
\hline & -3.59281500 & -1.31841900 & -0.70398100 \\
\hline & -3.93658800 & -0.30462200 & 0.20280100 \\
\hline & -3.48407700 & 1.42570000 & 1.50183000 \\
\hline & -4.82493400 & -2.09358400 & -1.01847700 \\
\hline & -5.57371400 & -1.42629500 & -1.45494600 \\
\hline & -4.62597900 & -2.90511800 & -1.71424300 \\
\hline & -5.24535600 & -2.50469100 & -0.09643900 \\
\hline & -1.89224200 & -2.56972600 & -2.12299000 \\
\hline & -2.68774200 & -3.27367900 & -2.35909300 \\
\hline & -1.53872000 & -2.12313700 & -3.05734200 \\
\hline & -1.05730900 & -3.12483700 & -1.68681300 \\
\hline & 4.22960000 & -0.63339300 & -0.74406400 \\
\hline & 4.57602100 & -1.61167200 & -1.10461600 \\
\hline & 3.98242200 & 0.25296700 & -1.97272100 \\
\hline & 3.39911600 & -0.30712100 & -2.71623400 \\
\hline & 4.93812400 & 0.50545200 & -2.44446600 \\
\hline & -0.69997800 & -1.86899200 & 2.24572700 \\
\hline & -1.17240200 & -1.10335900 & 2.87096500 \\
\hline & -1.42128600 & -2.15565200 & 1.46767700 \\
\hline & -0.54713200 & -2.74840400 & 2.87364100 \\
\hline & -1.59833300 & 0.93238900 & -1.14257700 \\
\hline
\end{tabular}

$\begin{array}{lrrr}\text { O } & -1.03829900 & 3.28952500 & -1.12865700 \\ \text { H } & -1.17599400 & 2.22582200 & -1.25324300 \\ \text { H } & -1.35170100 & 3.79046700 & -1.89558000 \\ \text { O } & -2.05824500 & 3.72080400 & 1.06003200 \\ \text { H } & -2.27786500 & 4.56617100 & 1.46942000 \\ \text { H } & -2.65092600 & 3.01712500 & 1.40515500 \\ \text { H } & -1.51040600 & 3.58193400 & -0.22215300\end{array}$

TS_2 b ' -3 '

Sum of electronic and thermal Free Energies $=-1270.272006$

\begin{tabular}{|c|c|c|c|}
\hline C & 2.63802100 & -0.49680300 & -0.87226300 \\
\hline $\mathbf{H}$ & 2.03518800 & 0.11114800 & -1.56602200 \\
\hline C & 1.90829000 & 0.96244000 & 1.04477900 \\
\hline $\mathbf{H}$ & 1.34231900 & 1.60964000 & 0.36712700 \\
\hline H & 1.42136600 & 1.00518100 & 2.02790600 \\
\hline C & 3.32791700 & 1.52473300 & 1.13808900 \\
\hline $\mathbf{H}$ & 3.93723300 & 0.92325900 & 1.82229800 \\
\hline $\mathbf{H}$ & 3.29447700 & 2.52846800 & 1.57893300 \\
\hline C & 5.20825600 & -0.62694500 & -0.27907800 \\
\hline H & 5.24163600 & -1.65917000 & -0.63572900 \\
\hline H & 6.14721600 & -0.15361200 & -0.58038600 \\
\hline H & 5.20301800 & -0.64726500 & 0.8135 \\
\hline C & 2.42317600 & -1.47769100 & 1.49116800 \\
\hline H & 1.99661100 & -1.31969300 & 2.48618500 \\
\hline H & 2.21552400 & -2.51032500 & 1.19674500 \\
\hline H & 3.50303200 & -1.37500700 & 1.58725200 \\
\hline C & 1.86117900 & -0.46628800 & 0.47143300 \\
\hline C & 0.38851500 & -0.88088800 & 0.18725800 \\
\hline C & 1.29177400 & -2.51632900 & -1.46000400 \\
\hline $\mathbf{H}$ & 1.13888100 & -3.37875900 & -2.10644500 \\
\hline C & 2.66011600 & -1.91260900 & -1.43951500 \\
\hline $\mathbf{H}$ & 3.06814900 & -1.90140900 & -2.45732700 \\
\hline H & 3.32666500 & -2.56371400 & -0.85844100 \\
\hline C & 0.24984100 & -2.07051700 & -0.74955400 \\
\hline C & -0.47361600 & -1.00236700 & 1.46223200 \\
\hline H & -0.65707000 & -2.04114600 & 1.74239300 \\
\hline $\mathbf{H}$ & 0.00942600 & -0.50202600 & 2.30285000 \\
\hline 0 & -0.30812300 & 0.23288100 & -0.56880600 \\
\hline C & -1.77266000 & -0.24284400 & 1.19394800 \\
\hline C & -3.01621100 & -1.12074800 & 0.97282700 \\
\hline C & -2.53043000 & 0.97319500 & -0.95448800 \\
\hline C & -3.71077300 & 0.34134300 & -0.81114100 \\
\hline 0 & -3.94560500 & -0.67250400 & 0.10081500 \\
\hline 0 & -3.23063200 & -2.07527400 & $1.0000 \mathrm{~s}$ \\
\hline C & -4.93456200 & 0.55461800 & -1.63159600 \\
\hline $\mathbf{H}$ & -5.78177300 & 0.73515700 & -0.96387300 \\
\hline $\mathbf{H}$ & -4.82464400 & 1.40194100 & -2.30525500 \\
\hline $\mathbf{n}$ & -5.15804000 & -0.34760500 & -2.20894900 \\
\hline C & -2.23240700 & 1.99954000 & -2.01579400 \\
\hline $\mathbf{H}$ & -1.15440800 & 2.04960600 & -2.18654300 \\
\hline H & -2.70564700 & 1.75075600 & -2.96616900 \\
\hline $\mathbf{H}$ & -2.57217500 & 2.99007500 & -1.69984300 \\
\hline C & 4.03886800 & 0.16839600 & -0.87808500 \\
\hline $\mathbf{H}$ & 4.27756400 & 0.29445800 & -1.94272400 \\
\hline C & 3.96336900 & 1.56824600 & -0.25259800 \\
\hline H & 3.35632200 & 2.21609700 & -0.90308600 \\
\hline H & 4.96439500 & 2.01024100 & -0.20896400 \\
\hline 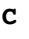 & -1.11830800 & -2.68320400 & -0.90265600 \\
\hline & 8632 & 45716 & 017280 \\
\hline
\end{tabular}




$\begin{array}{lrrr}\text { H } & -1.85568600 & -1.93541300 & -1.22957400 \\ \text { H } & -1.10068400 & -3.45494700 & -1.67383500 \\ \text { O } & -2.15819800 & 0.62062000 & 2.24902500 \\ \text { H } & -2.45629000 & 0.08818700 & 3.00181000 \\ \text { C } & -1.45306500 & 0.56868400 & -0.06134100 \\ \text { O } & -1.15287000 & 2.30028300 & 0.69742800 \\ \text { H } & -1.25852700 & 2.27486900 & 1.66097500 \\ \text { H } & -0.34562500 & 2.80910000 & 0.43816900 \\ \text { O } & 0.82258500 & 3.90108000 & -0.15028900 \\ \text { H } & 1.77437700 & 3.88796400 & 0.00690000 \\ \text { H } & 0.58480300 & 4.80917900 & -0.37244300\end{array}$

TS_3 '-4a'

Sum of electronic and thermal Free Energies $=-1270.269198$
2.72856800
2.16311800
$-0.15544500$
$-0.79145900$
1.81883600
0.54819800
$-1.42447200$
1.23767500
0.89373500
1.32398900
1.31415600
1.66971000
0.80730200
3.20906500
0.69523600
2.28094400
3.80082700
1.46450700
1.60989400
3.11210300
0.75176000
2. 19591100
5.26324900
2. 36325500
2.22683400
5.37196600
$-0.30572400$
$-0.06190400$
6.19351400
$-1.24338400$
$-0.61210000$
5.18933300
0.25413700
$-0.19507100$
2.39571600
$-0.54398600$
1.00198400
1.95927000
$-1.56689700$
1.31023300
2.31337000
$2.18994100-2.53172700$
0.83780900
$3.47457500-1.49054600$
1.43934100
1.85137800
$-0.39209200$
0.47010100
0.40199000
$-0.76561500$
$-0.01592500$
1.51922200
$-2.07993900-1.83070900$
1.45601600
$-2.81764700$
$-2.62890200$
2.85499000
$-1.44674400$
$-1.59406600$
3.34077800
$-1.24107300$
$-2.55580000$
3.50608600
$-2.16625400$
$-1.08049300$
0.40873400
$-1.80148500$
$-1.13944400$
$-0.47629300-1.18517900$
1.19341200
$-0.61569800-2.26819700$
1.25346900
0.00819300
$-0.88260500$
2.12393500
$-0.32387700$
0.37887500
$-0.51506200$
$-1.87184200$
$-0.51045800$
1.27504400
$-2.95440100$
$-1.33364900$
0.57645400
$-2.75392900 \quad 1.43605200$
$-0.19316600$
$-3.61644500$
0.55198200
$-0.76279200$
$-3.67817900$
$-0.77105600$
$-0.42983400$
$-3.19830500$
$-2.44643100$
0.92990600
$-4.64697700$
0.83419800
$-1.79678800$
$-4.46496600$
0.18556000
$-2.65952900$
$-5.63160900$
$0.57112600-1.39846100$
$-4.64985700$
1.87172700
$-2.11733900$
$-2.65637700$
2.90173200
$-0.51991900$
$-2.83733100$
3.50506000
0.37300800
$-1.64899600$
3. 12878100
$-0.88431300$
$-3.37228600$
3.19883400
$-1.28272400$
4.09491400
$0.54863300-0.57584200$
4.39239300
0.88249300
$-1.57968800$
3.91756600
1.79671200

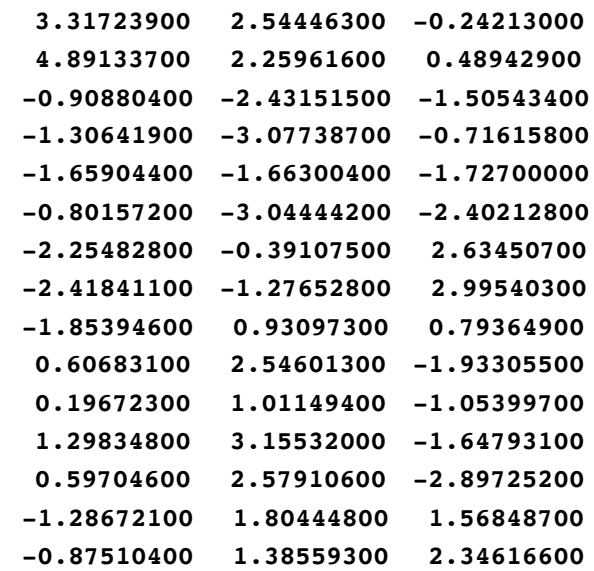

TS_ $4 \mathrm{~b}^{\prime}-5^{\prime}$

Sum of electronic and thermal Free Energies $=-1270.274448$

\begin{tabular}{lrrr}
\hline C & -2.83018300 & 0.00777100 & 0.26793100 \\
H & -2.43227700 & -0.19370500 & 1.27747000 \\
C & -2.00721600 & 2.79795400 & -0.10609000 \\
H & -2.41786500 & 2.98509600 & -1.10531000 \\
H & -1.51074100 & 3.72499400 & 0.19921700 \\
C & -4.84416500 & 1.44260100 & -0.65905200 \\
H & -5.32813700 & 0.53701500 & -1.03261400 \\
H & -5.63571200 & 2.09329700 & -0.27628600 \\
H & -4.39493900 & 1.96219500 & -1.50909900 \\
C & -1.93942000 & 0.37856600 & -2.10409000 \\
H & -1.12969100 & 0.82042600 & -2.69249700 \\
H & -2.14845500 & -0.61455300 & -2.51210600 \\
H & -2.82222600 & 0.99338000 & -2.27211800 \\
C & -1.58390000 & 0.32170300 & -0.60327200 \\
C & -0.55322800 & -0.84277700 & -0.41732600 \\
C & -2.50905200 & -2.38550600 & -0.38737000 \\
H & -2.67443700 & 2.32322200 & 1.87830200 \\
H & -2.90740800 & -3.39730600 & -0.44039900 \\
H & -3.8450
\end{tabular}




$\begin{array}{rrrr}\text { H } & -3.84021400 & 3.27171000 & 0.96043600 \\ \text { C } & -0.27557200 & -3.43015100 & -0.58478000 \\ \text { H } & 0.34296400 & -3.42844600 & -1.48578100 \\ \text { H } & 0.41201400 & -3.47999900 & 0.26700200 \\ \text { H } & -0.87238300 & -4.34372600 & -0.58120800 \\ \text { C } & 3.05198100 & 0.08306000 & -0.90779500 \\ \text { O } & 3.84263500 & -0.35235400 & -1.85515000 \\ \text { C } & -0.97969900 & 1.66533000 & -0.14440200 \\ \text { H } & -0.13952400 & 1.95082400 & -0.79432000 \\ \text { H } & -0.58187700 & 1.54569600 & 0.86895700 \\ \text { C } & 2.42959600 & 1.64602500 & 0.75600200 \\ \text { O } & 2.45080300 & -2.17109300 & -0.63669800 \\ \text { O } & 1.63759600 & 0.81791200 & 1.34852200 \\ \text { H } & -0.25458200 & -1.56322000 & 1.79837500 \\ \text { H } & 2.76404400 & -2.45384600 & 0.24461800 \\ \text { H } & 3.68102300 & -1.30767400 & -1.98927000 \\ \text { O } & -0.09256300 & -2.34449800 & 2.68912000 \\ \text { H } & 0.89995900 & -2.17738300 & 2.73794200 \\ \text { H } & -0.52473200 & -2.15553600 & 3.53322200\end{array}$

TS_5'-6a'

Sum of electronic and thermal Free Energies $=-1270.290704$

\begin{tabular}{|c|c|c|c|}
\hline C & -2.90641600 & 0.07129700 & 0.34075400 \\
\hline H & -2.46279800 & 0.10874800 & 1.34911400 \\
\hline C & -1.99328700 & 2.62313200 & -0.78198400 \\
\hline H & -2.45034400 & 2.56995600 & -1.77717800 \\
\hline H & -1.44212900 & 3.56848000 & -0.74948800 \\
\hline C & -4.90744300 & 1.32305000 & -0.84402900 \\
\hline H & -5.45172400 & 0.38085800 & -0.94378300 \\
\hline $\mathbf{H}$ & -5.64654500 & 2.09616300 & -0.61486700 \\
\hline H & -4.48319200 & 1.57428900 & -1.81936700 \\
\hline C & -2.12571000 & -0.23024000 & -2.08370000 \\
\hline H & -1.32321000 & -0.01221100 & -2.79467000 \\
\hline H & -2.41244500 & -1.27819800 & -2.20826100 \\
\hline H & -2.97953500 & 0.37958200 & -2.37444000 \\
\hline C & -1.69772000 & 0.08608100 & -0.63392300 \\
\hline C & -0.71093700 & -1.04369800 & -0.20771700 \\
\hline C & -2.71685200 & -2.41863700 & 0.31499400 \\
\hline H & -3.15568300 & -3.38881000 & 0.54134400 \\
\hline C & -3.65072800 & -1.25498100 & 0.23764400 \\
\hline H & -4.39679700 & -1.33132700 & 1.03789000 \\
\hline H & -4.21578400 & -1.32001100 & -0.70171700 \\
\hline C & -1.39204300 & -2.36174000 & 0.13109700 \\
\hline C & 0.49839800 & -1.17691000 & -1.17251100 \\
\hline H & 0.43497100 & -2.07762200 & -1.78594700 \\
\hline H & 0.54603600 & -0.31529500 & -1.83751100 \\
\hline 0 & -0.02836100 & -0.65310500 & 1.09740400 \\
\hline C & 1.77382100 & -1.21133400 & -0.31178300 \\
\hline C & 1.24976200 & -0.75028400 & 1.03456600 \\
\hline C & 3.28984600 & 0.87545100 & -0.78828100 \\
\hline 0 & 1.97192700 & -0.89693900 & 2.07945700 \\
\hline C & 3.07141400 & 3.13520600 & 0.37058500 \\
\hline H & 3.12917000 & 3.65228300 & -0.59103400 \\
\hline H & 2.38981000 & 3.66085500 & 1.03780400 \\
\hline H & 4.07879500 & 3.14168800 & 0.80059400 \\
\hline C & 4.42365900 & 1.45029500 & -1.60234900 \\
\hline $\mathbf{H}$ & 4.95009200 & 0.66268700 & -2.13867200 \\
\hline H & 4.05589400 & 2.16986000 & -2.34103600 \\
\hline H & 5.14557200 & 1.96650000 & -0.96470500 \\
\hline
\end{tabular}

$\begin{array}{rrrr}\text { C } & -3.86333900 & 1.29204700 & 0.28236700 \\ \text { H } & -4.43731700 & 1.23260500 & 1.21728600 \\ \text { C } & -3.06410300 & 2.60151300 & 0.30806600 \\ \text { H } & -2.57008500 & 2.71167900 & 1.28376100 \\ \text { H } & -3.74514500 & 3.45247000 & 0.20151800 \\ \text { C } & -0.54097900 & -3.58967900 & 0.33021300 \\ \text { H } & 0.04194900 & -3.85923600 & -0.55412600 \\ \text { H } & 0.17287000 & -3.45422600 & 1.15036900 \\ \text { H } & -1.17785200 & -4.43782000 & 0.58634700 \\ \text { C } & 2.91561000 & -0.41971900 & -0.95789300 \\ \text { O } & 3.55361400 & -1.15540100 & -1.87289100 \\ \text { C } & -1.01600600 & 1.46631000 & -0.56906500 \\ \text { H } & -0.19832700 & 1.52899600 & -1.29960100 \\ \text { H } & -0.56399000 & 1.59312100 & 0.41967600 \\ \text { C } & 2.60959900 & 1.71844400 & 0.18824600 \\ \text { O } & 2.21420500 & -2.55045000 & -0.16163600 \\ \text { O } & 1.66649700 & 1.30351600 & 0.88248500 \\ \text { H } & 0.33553600 & 0.76083800 & 4.25971500 \\ \text { H } & 2.83204100 & -2.61430300 & 0.58475000 \\ \text { H } & 3.31426600 & -2.09248900 & -1.77491200 \\ \text { O } & 1.08185500 & 0.78845300 & 3.64923900 \\ \text { H } & 1.57243600 & -0.35294200 & 2.87096600 \\ \text { H } & 0.95629100 & 1.52060700 & 3.02593900\end{array}$




$\begin{array}{rrrr}\text { C } & 4.48980700 & 0.67448800 & -0.57146400 \\ \text { H } & 5.07645200 & 0.28675900 & -1.41545000 \\ \text { C } & 3.95767400 & 2.05717700 & -0.97112200 \\ \text { H } & 3.52168900 & 2.00448200 & -1.97790100 \\ \text { H } & 4.78653000 & 2.77132800 & -1.02154700 \\ \text { C } & 0.23993000 & -3.34664700 & 0.20267500 \\ \text { H } & -0.38458800 & -3.31366800 & 1.10059300 \\ \text { H } & -0.42536300 & -3.24539400 & -0.66378800 \\ \text { H } & 0.67433000 & -4.34579000 & 0.14405600 \\ \text { C } & -2.25832900 & 0.86030600 & 0.03035000 \\ \text { O } & -1.49315000 & 1.94748500 & -0.05302400 \\ \text { C } & 1.69884100 & 1.57710500 & 0.01717100 \\ \text { H } & 0.91823700 & 1.95478800 & 0.68555600 \\ \text { H } & 1.26612400 & 1.55853600 & -0.99059500 \\ \text { C } & -3.91174100 & -0.78603400 & 0.22766000 \\ \text { O } & -2.55383000 & -1.42636500 & -0.06733600 \\ \text { H } & -1.98720500 & 2.79911200 & -0.01313200 \\ \text { O } & -4.75605900 & -1.21250700 & -0.76448100 \\ \text { H } & -4.69176700 & -0.64149700 & -1.54391500 \\ \text { C } & -4.37770700 & -1.34820700 & 1.54747400 \\ \text { H } & -5.32863100 & -0.88204300 & 1.81438200 \\ \text { H } & -4.52996900 & -2.42489100 & 1.45133400 \\ \text { H } & -3.64304300 & -1.14217300 & 2.32838300 \\ \text { H } & -2.40956000 & -1.55861400 & -1.26806300 \\ \text { O } & -2.62684000 & 4.40588400 & -0.03496700 \\ \text { H } & -2.52302000 & 5.00766000 & 0.71166500 \\ \text { H } & -2.54285600 & 4.94015500 & -0.83383000\end{array}$

TS_7'-8'

Sum of electronic and thermal Free Energies $=-1270 \cdot 300514$

$\begin{array}{lrrr}\text { C } & -3.32517200 & -0.36045500 & 0.47510100 \\ \text { H } & -2.88137200 & -0.48471100 & 1.47504800 \\ \text { C } & -3.01370600 & 2.53248700 & 0.09133000 \\ \text { H } & -3.47335600 & 2.64828800 & -0.89784700 \\ \text { H } & -2.68406300 & 3.53154200 & 0.39392300 \\ \text { C } & -5.56887000 & 0.69392000 & -0.42968000 \\ \text { H } & -5.89295500 & -0.28420300 & -0.79416800 \\ \text { H } & -6.45702300 & 1.20157800 & -0.04199700 \\ \text { H } & -5.22331100 & 1.27686000 & -1.28716500 \\ \text { C } & -2.54115300 & 0.14672400 & -1.91434300 \\ \text { H } & -1.80318000 & 0.68549400 & -2.51662100 \\ \text { H } & -2.61292400 & -0.87359300 & -2.30209300 \\ \text { H } & -3.50189000 & 0.63070500 & -2.08418500 \\ \text { C } & -2.17108500 & 0.17172100 & -0.41705500\end{array}$

\begin{tabular}{|c|c|c|c|}
\hline C & -0.95685800 & -0.78219400 & -0.23052500 \\
\hline C & -2.58204800 & -2.66475000 & -0.16228600 \\
\hline n & 2.78488500 & -3.73412100 & -0.19242400 \\
\hline 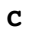 & -3.75471300 & -1.75047300 & 0.01141700 \\
\hline $\mathbf{H}$ & -4.44954800 & -2.18742900 & 0.73886800 \\
\hline $\mathbf{H}$ & -4.30850800 & -1.70033700 & -0.93565200 \\
\hline C & -1.31178200 & -2.26073700 & -0.26904300 \\
\hline 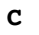 & 0.24592500 & -0.42253600 & -1.14866800 \\
\hline $\mathbf{H}$ & 0.37876400 & -1.13878300 & -1.95998400 \\
\hline $\mathbf{H}$ & 0.10532100 & 0.56678400 & -1.58461300 \\
\hline 0 & -0.40858700 & -0.57907200 & 1.14597000 \\
\hline $\mathbf{C}$ & 1.46968000 & -0.36912800 & -0.24978900 \\
\hline C & 0.88736500 & -0.51938700 & 1.17151100 \\
\hline C & 3.63437600 & 0.53954500 & -0.33437100 \\
\hline 0 & 1.56854800 & -0.58965800 & 2.18374400 \\
\hline C & 4.86457100 & 1.38866400 & -0.33237200 \\
\hline $\mathbf{H}$ & 5.49091900 & 1.18027900 & -1.20531900 \\
\hline $\mathbf{H}$ & 4.60776000 & 2.44916200 & -0.34631900 \\
\hline $\mathbf{H}$ & 5.46058700 & 1.19607100 & 0.56567900 \\
\hline C & -4.52740100 & 0.59360100 & 0.69460000 \\
\hline H & -5.05962100 & 0.16958300 & 100 \\
\hline C & -4.02817300 & 1.98812700 & 600 \\
\hline $\mathbf{H}$ & -3.54781700 & 1.93420700 & 2.083 \\
\hline $\mathbf{H}$ & -4.87937800 & 2.67050300 & 1.195 \\
\hline $\mathbf{C}$ & -0.17319900 & -3.24730900 & -0.327 \\
\hline $\mathbf{H}$ & 0.41498600 & -3.17290800 & -1.24785600 \\
\hline $\mathbf{H}$ & 0.52167900 & -3.10689400 & 0.51058600 \\
\hline $\mathbf{H}$ & -0.55673800 & -4.26663700 & -0.25932000 \\
\hline $\mathbf{C}$ & 2.32702100 & 0.87222300 & -0.32889100 \\
\hline 0 & 1.68208400 & 2.02240700 & -0.33518500 \\
\hline $\mathbf{C}$ & -1.79810000 & 1.60670600 & 0.01393100 \\
\hline H & -1.05131700 & 2.02905100 & -0.66877700 \\
\hline H & -1.33864300 & 1.57836400 & 1.00946900 \\
\hline $\mathbf{C}$ & 3.66399400 & -0.92364800 & -0.25267700 \\
\hline 0 & 2.42306800 & -1.42501200 & -0.43995900 \\
\hline 0 & 3.94648900 & -1.15474700 & 1.47233400 \\
\hline H & 4.13377300 & -2.07860600 & 1400 \\
\hline C & 4.76222600 & -1.75085800 & -0.83620100 \\
\hline $\mathbf{H}$ & 5.72994800 & -1.42875500 & -0.44895600 \\
\hline $\mathbf{H}$ & 4.60303300 & -2.81116400 & -0.62918800 \\
\hline $\mathbf{H}$ & 4.74774400 & -1.60548300 & -1.91989400 \\
\hline $\mathbf{H}$ & 3.05135100 & -0.88725300 & 1.90293000 \\
\hline $\mathbf{H}$ & 2.26127400 & 2.82363900 & -0.28405900 \\
\hline 0 & 3.07910900 & 4.29605700 & -0.17711000 \\
\hline $\mathbf{H}$ & 3.03386600 & 4.80259100 & 0.64298200 \\
\hline $\mathbf{H}$ & 3.02243400 & 4.93291900 & -0.89978300 \\
\hline
\end{tabular}




\section{Supplementary References}

(1) Jin, F. J.; Maruyama, J.; Juvvadi, P. R.; Arioka, M.; Kitamoto, K., Development of a novel quadruple auxotrophic host transformation system by $\arg B$ gene disruption using adeA gene and exploiting adenine auxotrophy in Aspergillus oryzae. FEMS Microbiol. Lett. 2004, 239, 79-85.

(2) Fujii, T.; Yamaoka, H.; Gomi, K.; Kitamoto, K.; Kumagai, C., Cloning and nucleotide sequence of the ribonuclease T1 gene (rntA) from Aspergillus oryzae and its expression in Saccharomyces cerevisiae and Aspergillus oryzae. Biosci., Biotechnol., Biochem. 1995, 59, 1869-1874.

(3) Wei, X.; Chen, X.; Chen, L.; Yan, D.; Wang, W.-G.; Matsuda, Y., Heterologous biosynthesis of tetrahydroxanthone dimers: Determination of key factors for selective or divergent synthesis. J. Nat. Prod. 2021, 1544-1549.

(4) Yamada, O.; Na Nan, S.; Akao, T.; Tominaga, M.; Watanabe, H.; Satoh, T.; Enei, H.; Akita, O., dffA Gene from Aspergillus oryzae encodes L-ornithine $N^{5}$-oxygenase and is indispensable for deferriferrichrysin biosynthesis. J. Biosci. Bioeng. 2003, 95, 82-88.

(5) Yamada, O.; Lee, B. R.; Gomi, K.; Iimura, Y., Cloning and functional analysis of the Aspergillus oryzae conidiation regulator gene brlA by its disruption and misscheduled expression. J. Biosci. Bioeng. 1999, 87, 424-429.

(6) Jin, F.; Maruyama, J.; Juvvadi, P.; Arioka, M.; Kitamoto, K., Adenine auxotrophic mutants of Aspergillus oryzae: Development of a novel transformation system with triple auxotrophic hosts. Biosci., Biotechnol., Biochem. 2004, 68, 656662 .

(7) Kubodera, T.; Yamashita, N.; Nishimura, A., Transformation of Aspergillus sp. and Trichoderma reesei using the pyrithiamine resistance gene (ptrA) of Aspergillus oryzae. Biosci., Biotechnol., Biochem. 2002, 66, 404-406.

(8) Matsuda, Y.; Bai, T.; Phippen, C. B. W.; Nødvig, C. S.; Kjærbølling, I.; Vesth, T. C.; Andersen, M. R.; Mortensen, U. H.; Gotfredsen, C. H.; Abe, I.; Larsen, T. O., Novofumigatonin biosynthesis involves a non-heme iron-dependent endoperoxide isomerase for orthoester formation. Nat. Commun. 2018, 9, 2587.

(9) Cohen, D. R.; Townsend, C. A., A dual role for a polyketide synthase in dynemicin enediyne and anthraquinone biosynthesis. Nat. Chem. 2018, 10, 231-236.

(10) Frisch, M. J.; Trucks, G. W.; Schlegel, H. B.; Scuseria, G. E.; Robb, M. A.; Cheeseman, J. R.; Scalmani, G.; Barone, V.; Petersson, G. A.; Nakatsuji, H.; Li, X.; Caricato, M.; Marenich, A. V.; Bloino, J.; Janesko, B. G.; Gomperts, R.; Mennucci, B.; Hratchian, H. P.; Ortiz, J. V.; Izmaylov, A. F.; Sonnenberg, J. L.; Williams; Ding, F.; Lipparini, F.; Egidi, F.; Goings, J.; Peng, B.; Petrone, A.; Henderson, T.; Ranasinghe, D.; Zakrzewski, V. G.; Gao, J.; Rega, N.; Zheng, G.; Liang, W.; Hada, M.; Ehara, M.; Toyota, K.; Fukuda, R.; Hasegawa, J.; Ishida, M.; Nakajima, T.; Honda, Y.; Kitao, O.; Nakai, H.; Vreven, T.; Throssell, K.; Montgomery Jr., J. A.; Peralta, J. E.; Ogliaro, F.; Bearpark, M. J.; Heyd, J. J.; Brothers, E. N.; Kudin, K. N.; Staroverov, V. N.; Keith, T. A.; Kobayashi, R.; Normand, J.; Raghavachari, K.; Rendell, A. P.; Burant, J. C.; Iyengar, S. S.; Tomasi, J.; Cossi, M.; Millam, J. M.; Klene, M.; Adamo, C.; Cammi, R.; Ochterski, J. W.; Martin, R. L.; Morokuma, K.; Farkas, O.; Foresman, J. B.; Fox, D. J. Gaussian 16 Rev. C.01, Wallingford, CT, 2016.

(11) Zhao, Y.; Truhlar, D. G., The M06 suite of density functionals for main group thermochemistry, thermochemical 
kinetics, noncovalent interactions, excited states, and transition elements: two new functionals and systematic testing of four M06-class functionals and 12 other functionals. Theor. Chem. Acc. 2008, 120, 215-241.

(12) (a) Fukui, K., The path of chemical reactions - the IRC approach. Acc. Chem. Res. 1981, 14, 363-368; (b) Page, M.; Doubleday, C.; Jr., J. W. M., Following steepest descent reaction paths. The use of higher energy derivatives with ab initio electronic structure methods. J. Chem. Phys. 1990, 93, 5634-5642; (c) Ishida, K.; Morokuma, K.; Komornicki, A., The intrinsic reaction coordinate. An ab initio calculation for $\mathrm{HNC} \rightarrow \mathrm{HCN}$ and $\mathrm{H}^{-}+\mathrm{CH}_{4} \rightarrow \mathrm{CH}_{4}+\mathrm{H}^{-} J$. Chem. Phys. 1977, 66, 2153-2156; (d) Gonzalez, C.; Schlegel, H. B., Reaction path following in mass-weighted internal coordinates. J. Phys. Chem. 1990, 94, 5523-5527.

(13) (a) Maeda, S.; Osada, Y.; Morokuma, K.; Ohno, K., GRRM11, Version 11.03. 2012; (b) Ohno, K.; Maeda, S., A scaled hypersphere search method for the topography of reaction pathways on the potential energy surface. Chem. Phys. Lett. 2004, 384, 277-282; (c) Maeda, S.; Ohno, K., Global mapping of equilibrium and transition structures on potential energy surfaces by the scaled hypersphere search method: Applications to ab initio surfaces of formaldehyde and propyne molecules. J. Phys. Chem. A 2005, 109, 5742-5753; (d) Ohno, K.; Maeda, S., Global reaction route mapping on potential energy surfaces of formaldehyde, formic acid, and their metal-substituted analogues. J. Phys. Chem. A 2006, 110, 89338941.

(14) Tamano, K.; Cox, R. S.; Tsuge, K.; Miura, A.; Itoh, A.; Ishii, J.; Tamura, T.; Kondo, A.; Machida, M., Heterologous production of free dihomo- $\gamma$-linolenic acid by Aspergillus oryzae and its extracellular release via surfactant supplementation. J. Biosci. Bioeng. 2019, 127, 451-457.

(15) Al Abdallah, Q.; Ge, W.; Fortwendel, J. R., A simple and universal system for gene manipulation in Aspergillus fumigatus: In vitro-assembled Cas9-guide RNA ribonucleoproteins coupled with microhomology repair templates. mShere 2017, 2, e00446-17.

(16) Fujimoto, H.; Negishi, E.; Yamaguchi, K.; Nishi, N.; Yamazaki, M., Isolation of new tremorgenic metabolites from an ascomycete, Corynascus setosus. Chem. Pharm. Bull. 1996, 44, 1843-1848.

(17) Macías, F. A.; Varela, R. M.; Simonet, A. M.; Cutler, H. G.; Cutler, S. J.; Dugan, F. M.; Hill, R. A., Novel bioactive breviane spiroditerpenoids from Penicillium brevicompactum Dierckx. J. Org. Chem. 2000, 65, 9039-9046.

(18) Kwon, J.; Seo, Y. H.; Lee, J.-E.; Seo, E.-K.; Li, S.; Guo, Y.; Hong, S.-B.; Park, S.-Y.; Lee, D., Spiroindole alkaloids and spiroditerpenoids from Aspergillus duricaulis and their potential neuroprotective effects. J. Nat. Prod. 2015, 78, 25722579.

(19) Gohbara, M.; Hyeon, S.-B.; Suzuki, A.; Tamura, S., Isolation and structure elucidation of colletopyrone from Colletotrichum nicotianae. Agric. Biol. Chem. 1976, 40, 1453-1455.

(20) Yaegashi, J.; Romsdahl, J.; Chiang, Y.-M.; Wang, C. C., Genome mining and molecular characterization of the biosynthetic gene cluster of a diterpenic meroterpenoid, 15-deoxyoxalicine B, in Penicillium canescens. Chem. Sci. 2015, 6, 6537-6544.

(21) Kelley, L. A.; Mezulis, S.; Yates, C. M.; Wass, M. N.; Sternberg, M. J. E., The Phyre2 web portal for protein modeling, prediction and analysis. Nat. Protoc. 2015, 10, 845-858. 
(22) Zhang, C.; Freddolino, P. L.; Zhang, Y., COFACTOR: Improved protein function prediction by combining structure, sequence and protein-protein interaction information. Nucleic Acids Res. 2017, 45, W291-W299. 